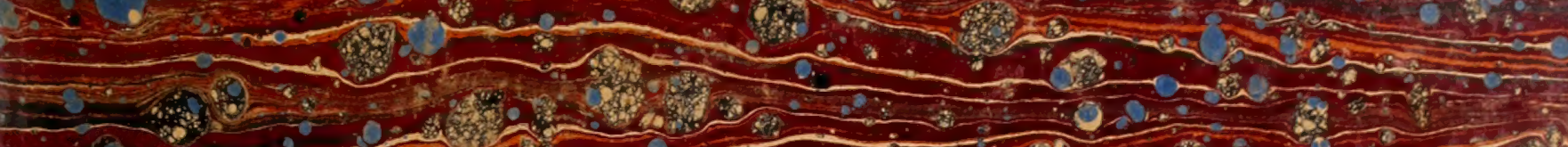

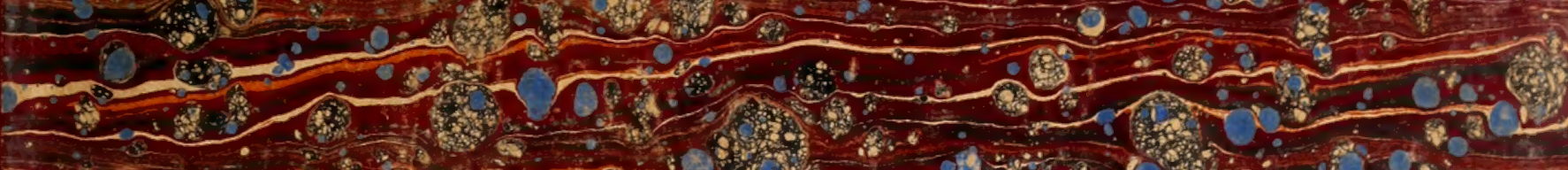

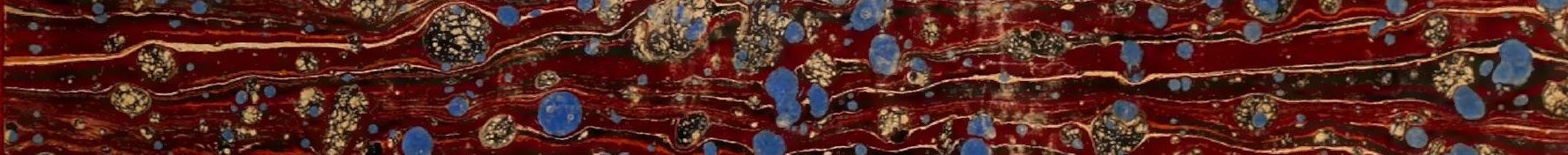

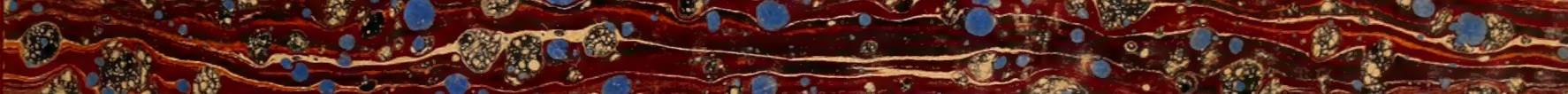

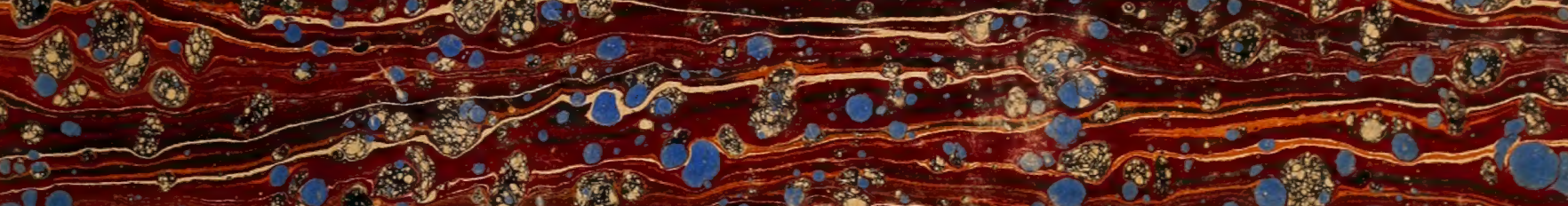

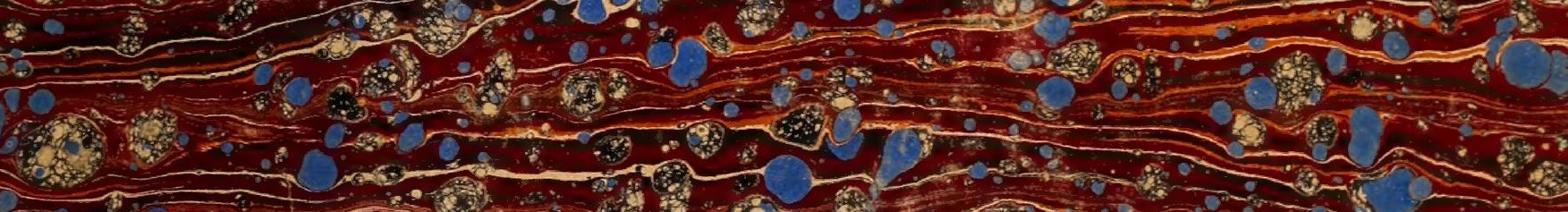

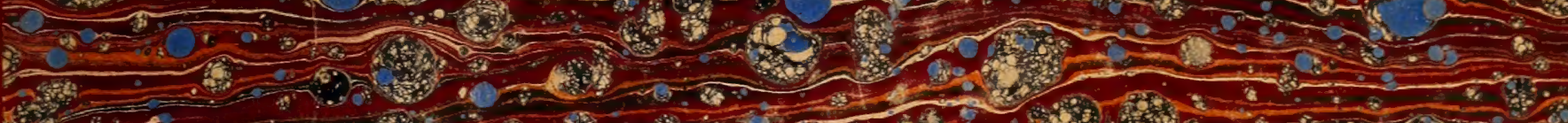

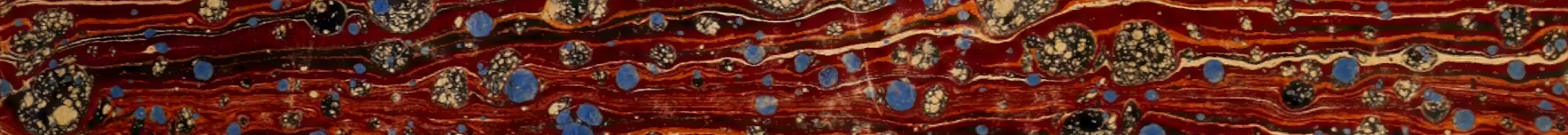

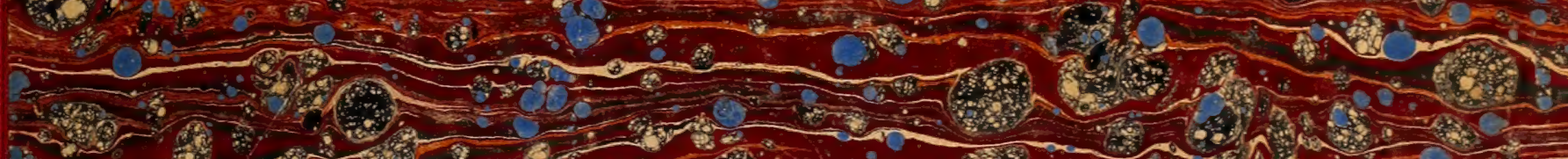

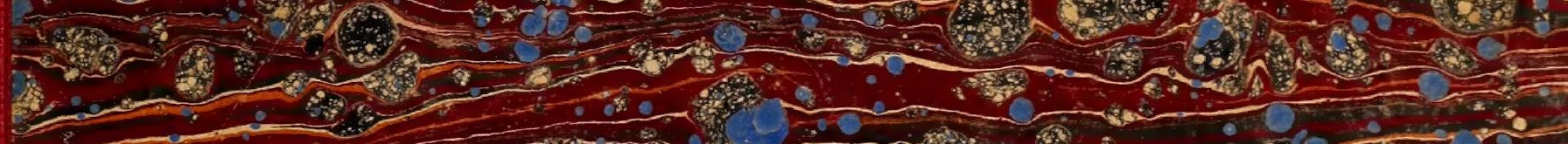
W.

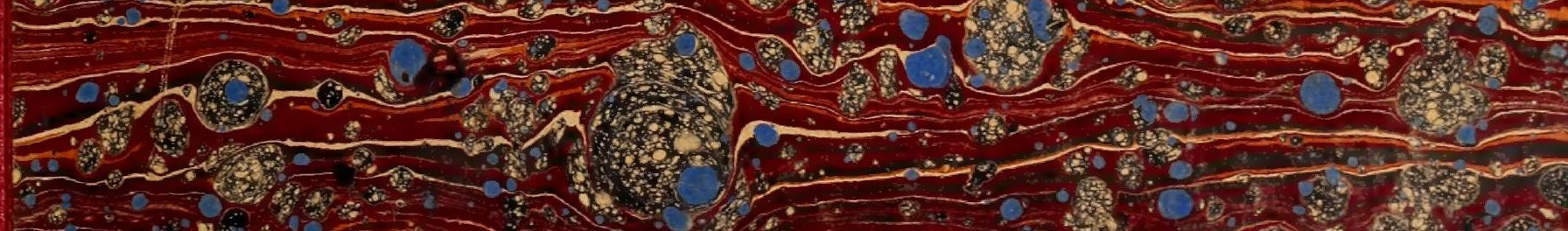

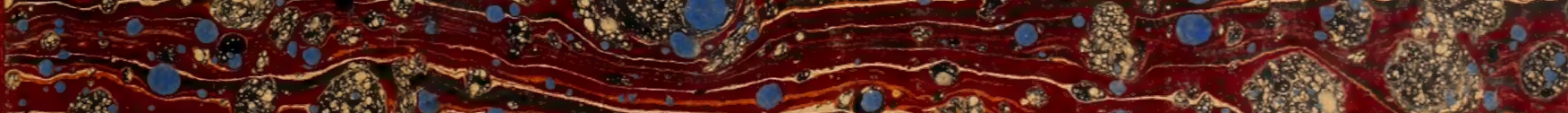

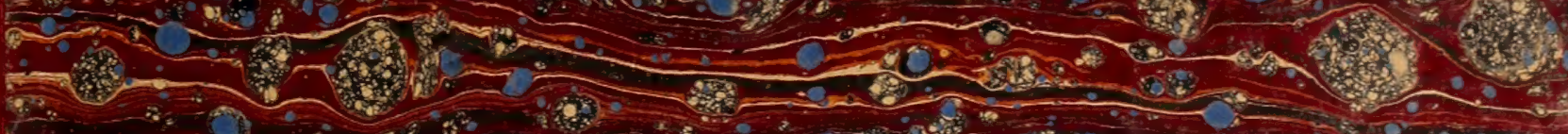



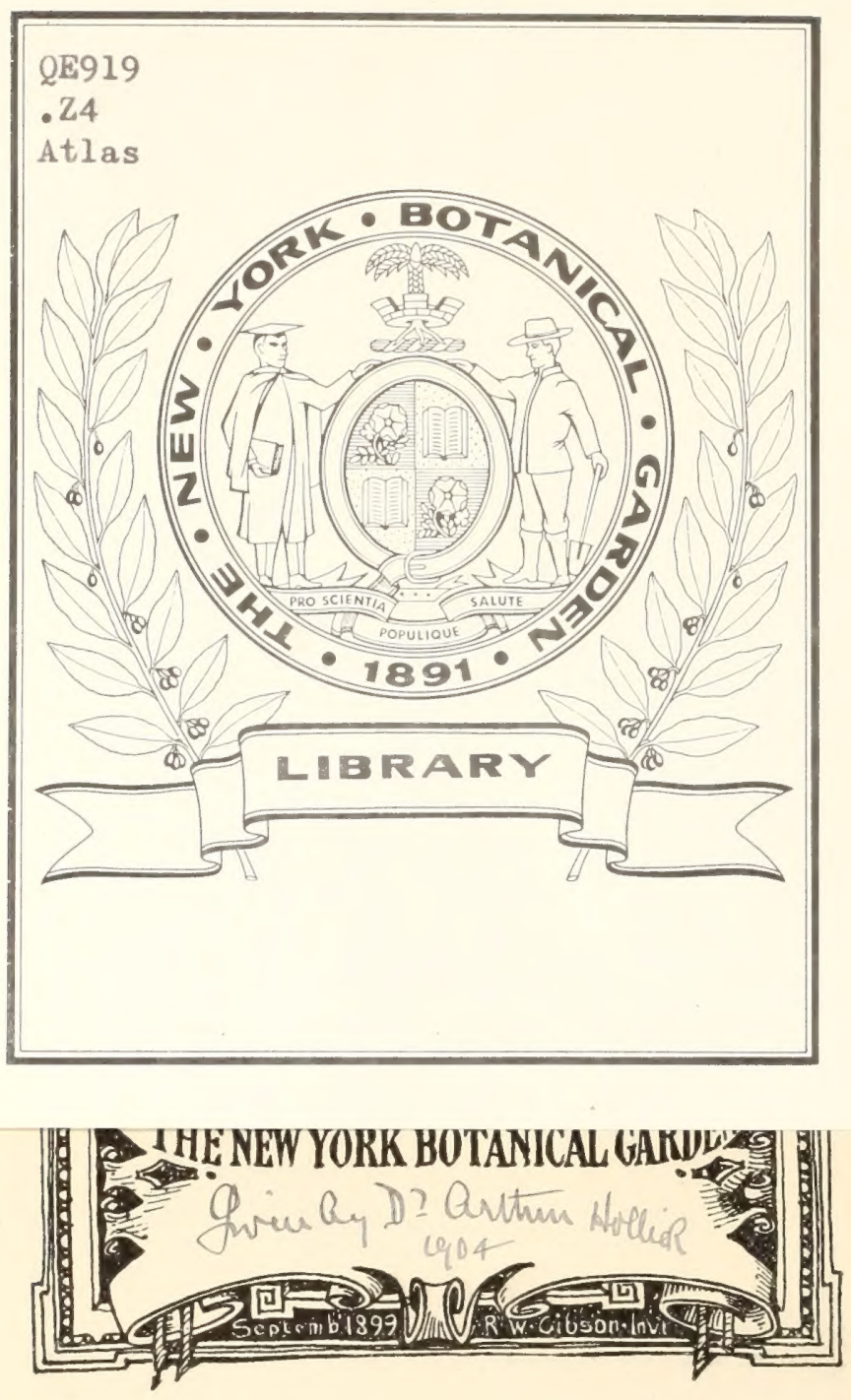






\section{MINISTERE DES TRAVAUX PUBLICS}

\section{ÉTUDES}

DES

\section{GITTES MINÉRIUX \\ DE LA FRANCE}

publiées sous les auspices de M. Le Ministre des travaux publics par le Service des Topographies solterkaines

COLONIES FRANGAISES

FLORE FOSSILE

DES

GÎTES DE CHARBON DU TONKIN

PAR

R. ZEILLER

INGÉNIEUR EV CHEF DES MINES, MEMBRE DE L'INSTITUT

PUbLIÉE AVEG LA PARTICIPATION DU GOUVERNEMENT DE LIINDO-CHINE

\section{ATLAS}

PHOTOTYPIES DE L. SOHIER

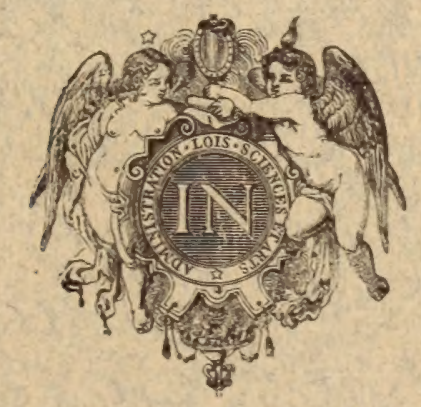

PARIS

IMPRIMERIE NATIONALE 



\section{FLORE FOSSILE}

DES

GİTES DE CHARBON DU TONKIN

ATLAS 



\section{ÉTUDES}

\section{GİTES MINÉRAUX DE LA FRANCE}

publiées sous les auspices de M. Le Ministre des travaux publics par le Service des Topographies souterraines

COLONIES FRANCAISES

FLORE FOSSILE DES

\section{GÎTES DE GHARBON DU TONKIN}

PAP

R. ZEILLER

INGÉNIEUR EN CHEF DES Mines, MEMBRE DE L'INSTITUT

Publiée AVEC LA PARTicipation DU GOUVERNeMENT DE L'INDO-GHINE
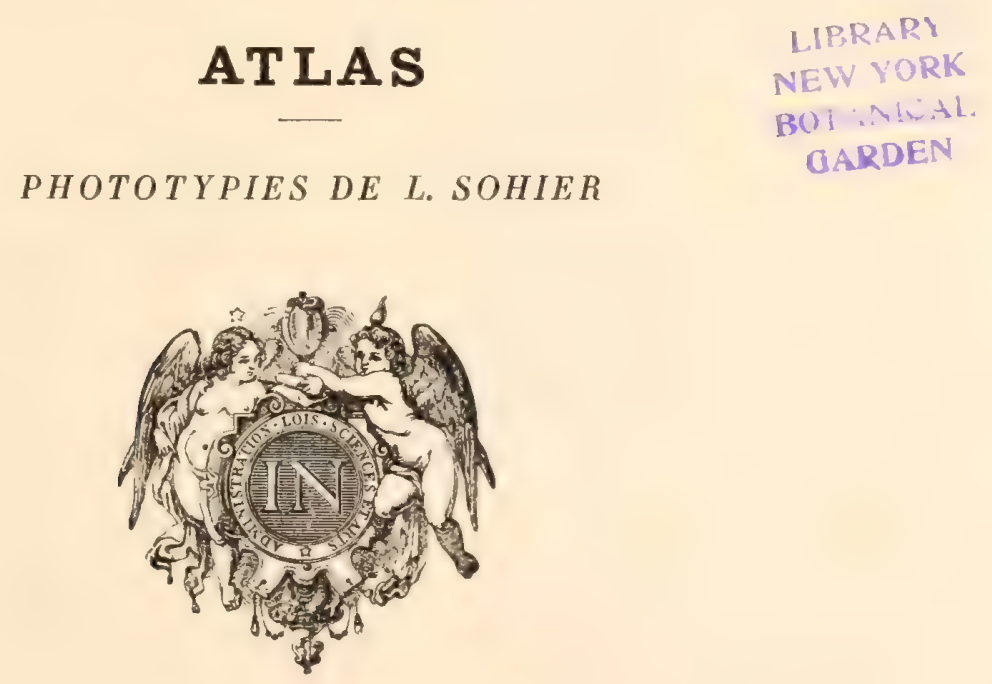

PARIS

IMPRIMERIE NATIONALE 



\section{TABLE ALPHABÉTIQUE}

\section{DES ESPĖCES FIGURÉES.}

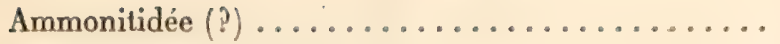

Angiopteris (?)

Annulariopsis inopinata, nov. gen., n. sp.

Baiera Guithaumati n. sp.

Cladophlebis ef. lobifolia Phillips (sp.)

Clad. nebbensis Brongniart (sp.)..............

Clad. Raciborskii n. sp. ..................

Clad. (Todea) Røesserti PresL (sp.)..............

Clathropteris platyphylla Goeppent (sp.).........

Conites Leclerei n. sp. . . . . . . . . . . . . . . . .

Conites sp........................

Conites sp. (cf. Kaidacarpum sibiricum HeER) .....

Ctenopteris Sarrani n. sp. . . . . . . . . . . . . .

Cycadites Saladini ZerLler. . . . . . . . . . . . .

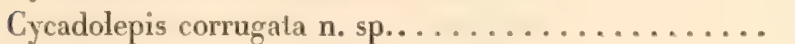

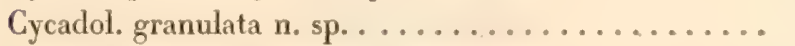

Cycadol. cf. villosa Saponta ...............

Danæopsis cf. Hughesi Feistuantel. . . . . . . . . . .

Dictyophyllum Fuchsi ZerLLer...............

Dictyoph. Nathorsti n. sp.................
PI. LIII, fig. 4.

PI. LI, fig. 1.

Pl. XXXV, fig. 2-7.

P1. L, fig. 16-19.

P1. IV, fig. 1.

P1. IV, fig. 2-4.

P1. V, fig. 1.

Pl. II, fig. 1-7; pl. III, fig. 1-3 ; pl. LIV, fig. 1, 2 .

Pl. XXVII, fig. 2, 3; pl. XXVIII, fig. 1, 2; pl. XXIX, fig. 1-4; pl. XXX, fig. $1-8$; pl. XXXI, fig. 1; pl. XXXIIXXXIII, fig. 1; pl. XXXIV, fig. 1; pl. LVI, fig. 4.

Pl. L, fig. 15.

P1. L, fig. 9-12.

P1. L, fig. 13,14 .

PI. VI-VII, fig. 1; pl. VIII, fig. 1, 2.

PI. XLI, fig. 1-4.

Pl. XLIV, fig. 1 ; pl. L, fig. 1-4.

P1. L, fig. 5.

P1. L, fig. 6 .

P1. IX, fig. 1.

PI. XVIII, fig. 1, 3.

PI. XXIII, fig. 1; pl. XXIV, fig. 1; pl. XXV, fig. 1-6; pl. XXVI, fig. 1-3; pl. XXVII, fig. 1; pl. XXVIII, fig. 3 ; pl. LVI, fig. 3. 


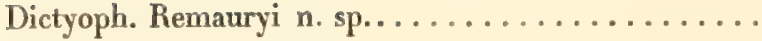

Dictyoph. Sarrani n. sp.

Écailles d'attribution incertaine.

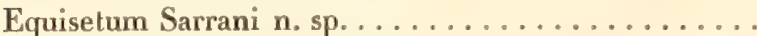

Etoblattina brevis Scudder (n. sp.) . . . . . . . .

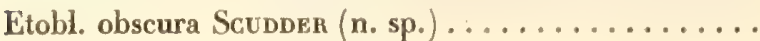

Ficus Beauveriei n. sp..................

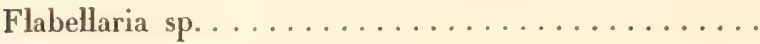

Fruit (ou graine) d'attribution incertaine.........

Gerablattina elegans Scudder (n. sp.)...........

Glossopteris angustifolia BrongNiART............

Gloss. indica Schimper....................

Nœggerathiopsis Hislopi Bunbury (sp.)..........

Nœggerathiopsis Hislopi ( $(?)$ [rameaux]

Organes d'attribution problématique. ...........

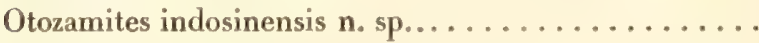

Otoz. rarinervis Feistmantel

Pałæovittaria Kurzi Feistmantel .............

Pecopteris adumbrata n. sp..................

Pec. (Asterotheca) Cottoni n. sp...............

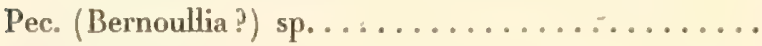

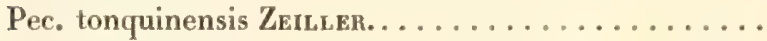

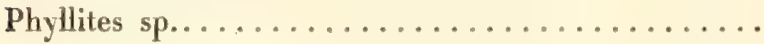

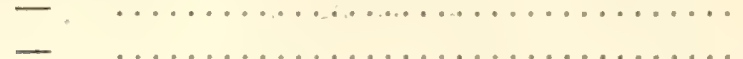

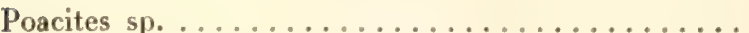

Podozamites distans PresL (sp.).............

Podoz. Schenki Heer... . . . . . . . . . . . . . . . .

Pterophyllum æquale Brongniakt (sp.)...........

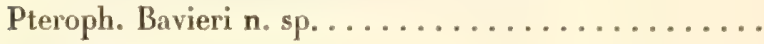

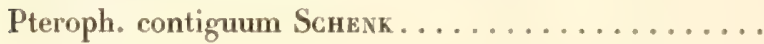

Pteroph. (Anomozamites) inconstans Bradn (sp.)....

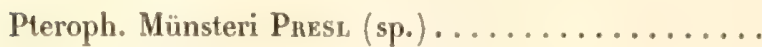

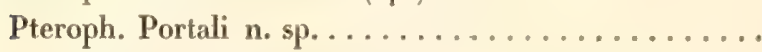

Pteroph. (Anomozamites) Schenki ZerLler.........

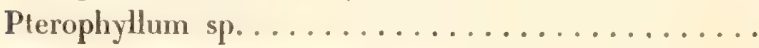

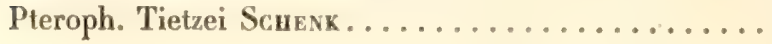

P1. XIX, fig. 1, 2 ; pl. XX, fig. 1-4; pl. XXI, fig. 1, 2.

Pl. XXII, fig. 1 .

Pl. L, fig. 8 .

P1. XXXIX, fig. 1-13.

PI. LIII, fig. 3.

P1. LIII, fig. 2 .

Pl. LI, fig. 4-13.

Pl. LII, fig. 1.

PI. LII, fig. 7 .

Pl. LIII, fig. 1.

PI. LVI, fig. 2.

Pl. XVI, fig. $2-5$; pl. LVI, fig. 1

Pl. XL, fig. 1-6.

Pl. XL, fig. 7-9.

Pl. L, fig. 20.

Pl. XLIII, fig. I.

Pl. XLIII, fig. 2.

Pl. XVI, fig. 1.

Pl. I, fig. 3.

PI. I, fig. 4-9.

Pl. I, fig。 14-16.

PI. 1, fig. 10-13.

P1. LII, fig. 3.

Pl. LII, fig. 4.

PI. LII, fig. 5.

PI. LII, fig. 6.

PI. LII, fig. 2.

PI. XLII, fig。 1-4.

P1. XLII, fig. $5,6$.

PI. XLIX, fig. 4-7.

P1. XLIX, fig. 1-3.

P1. XLVIII, fig。 1-8.

Pl. XLIII, fig. 8; pl. XLIV, fig. 1-5; pl. LVI, fig. 6.

PI. XLV, fig. 1-5.

P1. XLVI, fig. 1-5.

Pl. XLIII, fig. 7 .

Pl. LVI, fig. 5.

PI. XLVII, fig. 1 . 
Ptilophyllum acutifolium Morris.

Salvinia formosa HeEr

Schizoneura Carrerei n. sp.

Selliguea sp

Sphenopteris cf. princeps Prest (sp.)

Spiropteris SсHнмеR.

Tæniopteris (?)

Tæniopteris ensis Oldhay.

Tæn. cf. immersa NATHoRst.................

Tæn. Jourdyi ZEILLER.

Tæn. Leclerei n. sp.

Tæn. cf. Mac Clellandi Oldham et Morris (sp.).....

Tæn. (Marattia) Münsteri Goeppert.............

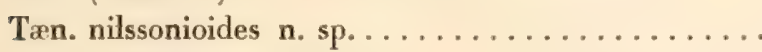

Tæn. spatulata Mac Gledland.................

Tæn. virgulata n. sp.

Unio sp.

Vivipara (Tylotoma) ef. Sturi NeUmayr

Williamsonia (??)

Woodwardites microlobus ScHENK.

Zamites truncatus n. sp.
Pl. LVI, fig. 7,8 .

P1. LI, fig. $2,3$.

Pl. XXXVI, fig. 1, 2 ; pl. XXXVII, fig. 1; pl. XXXVIII, fig。 $1-8$.

Pl. LI, fig. 1 .

Pl. I, fig. 1, 2.

Pl. XXXV, fig. 1.

PI. XII, fig. 5.

PI. IX, fig. 3.

Pl. LIV, fig. 5.

Pl. X, fig. 1-6; pl. XI, fig. 1-4; pl. XII, fig. 1-4, 6-8; pl. XIII, fig. 1-5.

Pl. LV, fig. 1-4.

PI. IX, fig. 3-5.

Pl. IX, fig. 6-8.

PI. XV, fig. 1-4.

PI. XIII, fig. 6-12.

PI. XIV, fig. 1-3.

P1. LIII, fig. 9-10.

P1. LIII, fig. 5-8.

P1. L, fig. 7.

Pl. XVII, fig. 1-5.

Pl. XLIII, fig. 3-6. 



\section{PLANCHE I}

GITES DE CHARBON DU TONKIN. 


\section{PLANCHE I.}

\section{EXPLICATION DES FIGURES.}

FIG. 1. - Sphenopteris cf. princeps PRESL, - Empreinte d'un fragment de fronde Mines de Kébao, système supérieur, couche $n^{\circ}$ 2, galerie $\mathbf{M}$.

FIG. I a. - Portion du même échantillon, grossie deux fois.

Fig. 2. - Sphenopteris cf. princeps PreSI. - Fragment de fronde. Kébao, puits Lanessan, travers-bancs Nord.

FIG. 2a. - Portion du même échantillon, grossie deux fois.

FIG. 3. - Pecopteris adumbrata n. sp. - Fragment de penne.

Mines de Hongaÿ: Hatou, au toit de la grande couche.

FIg. 3a. - Portion du même échantillon, grossie deux fois.

FIG. 4. - Pecopteris (Asterotheca) Cottoni n. sp. - Fragments de pennes. Hongay̆, vallée orientale de l'OEuf, galerie Léonice.

FIG. 4a, 4b. - Portions du même échantillon, grossies deux fois.

FIG. 5. - Pecopteris (Asterotheca) Gottoni n. sp. - Fragment de penne, à pinnules inférieures fertiles.

Mine de Nong-Sön (Annam).

FIG. 5 a. - Portion du même échantillon, grossie deux fois.

FIG. 6. - Pecopteris (Asterotheca) Gottoni n. sp. - Partie supérieure d'une penne. Hongaÿ, vallée orientale de l'OEuf, galerie Léonice.

FIG. 7. - Pecopteris (Asterotheca) Gottoni n. sp. - Empreinte d'une penne fertile Hongaÿ, vallée orientale de l'OEuf, galerie Léonice.

FIG. 7a. - Portion du même échantilton, grossie deux fois.

FIG. 8. - Pecopteris (Asterotheca) Cottoni n. sp. - Empreintes en creux de groupes de sporanges, grossies quinze fois.

Hongaÿ, mine de Carrère, au toit de la couche Marmottan.

FIG. 9. - Pecopteris (Asterotheca) Gọttoni n. sp. - Fragment de penne fertile.

Kébao, puits Lanessan.

FIG. 9a. - Portion du même échantillon, grossie deux fois.

FIG. 10. - Pecopteris tonquinensis ZeILlek. - Fragments de pennes (celui de gauche figuré Bull. Soc. Géol. de France, $3^{\mathrm{e}}$ série, t. XIV, pl. XXIV, fig. 2).

Bassin de Hongaÿ.

Fig. 11. - Pecopteris tonquinensis Zetller. - Fragment de penne (figuré Bull. Soc. Géol de France, $3^{\circ}$ série, t. XIV, pl. XXIV, fig. 4).

Bassin de Hongaÿ.

FIG. 11 a. - Portion du même échantillon, grossie deux fois.

Fig. 12. - Pecopteris tonquinensis ZeILIER. - Fragment de penne. Bassin de Hongaÿ.

Fig. 13. - Pecopteris tonquinensis Zeirler. - Fragment de penne (figuré Bull. Soc. Géol. de France, t. XIV, pl. XXIV, fig. 3).

Bassin de Hongaÿ.

FIG. $13 a_{0}$ - Portion du même échantillon, grossie deux fois.

FIG. 14 à 16. - Pecopteris (Bernoullia?) sp. - Fragments de pennes.

Hongaÿ, aflleurements du chemin des Singes.

Fig. 14 a et 15 a. - Portions des échantillons fig. 14 et fig. 15 , grossies deux fois. 
Pl. I
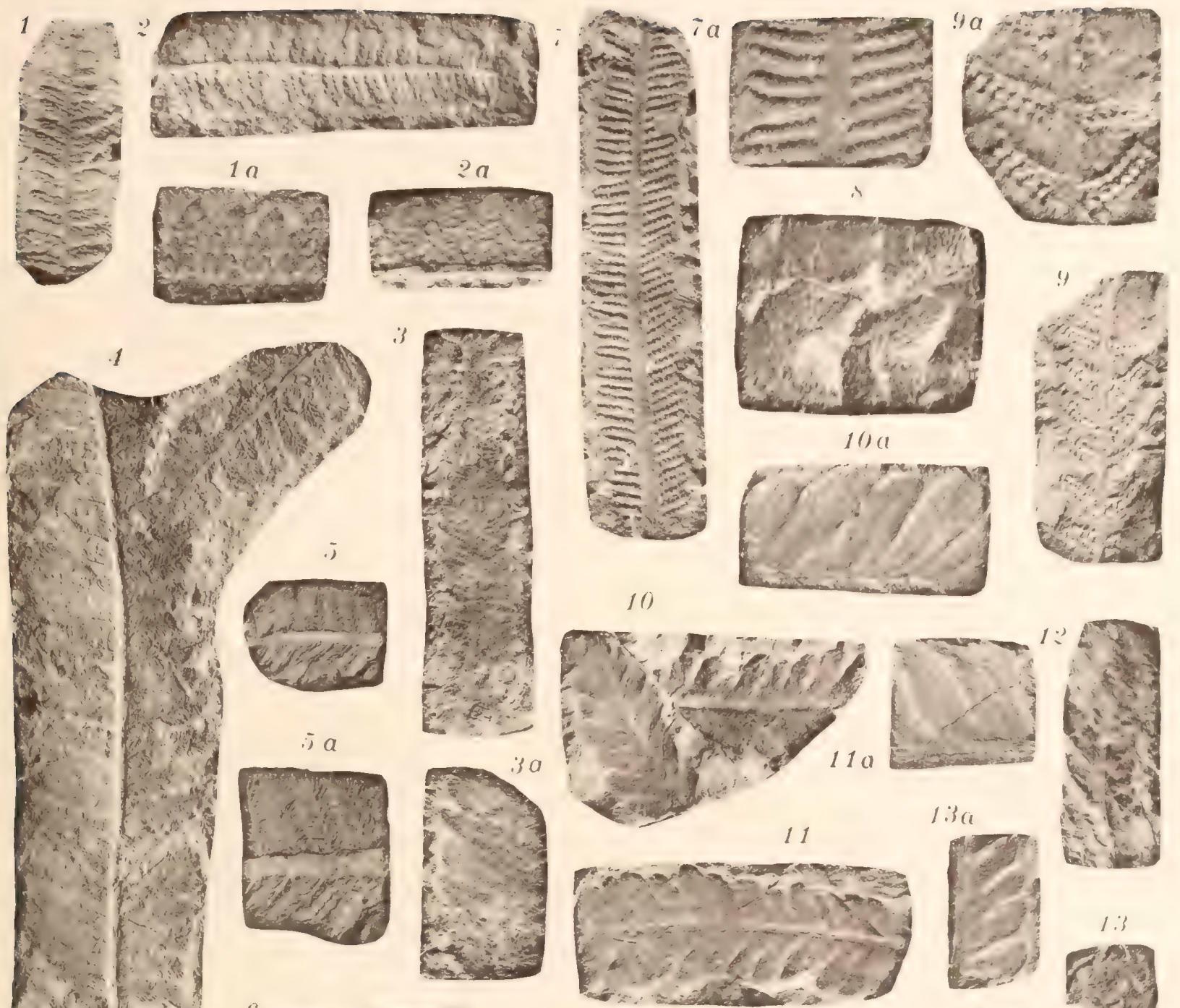

10)
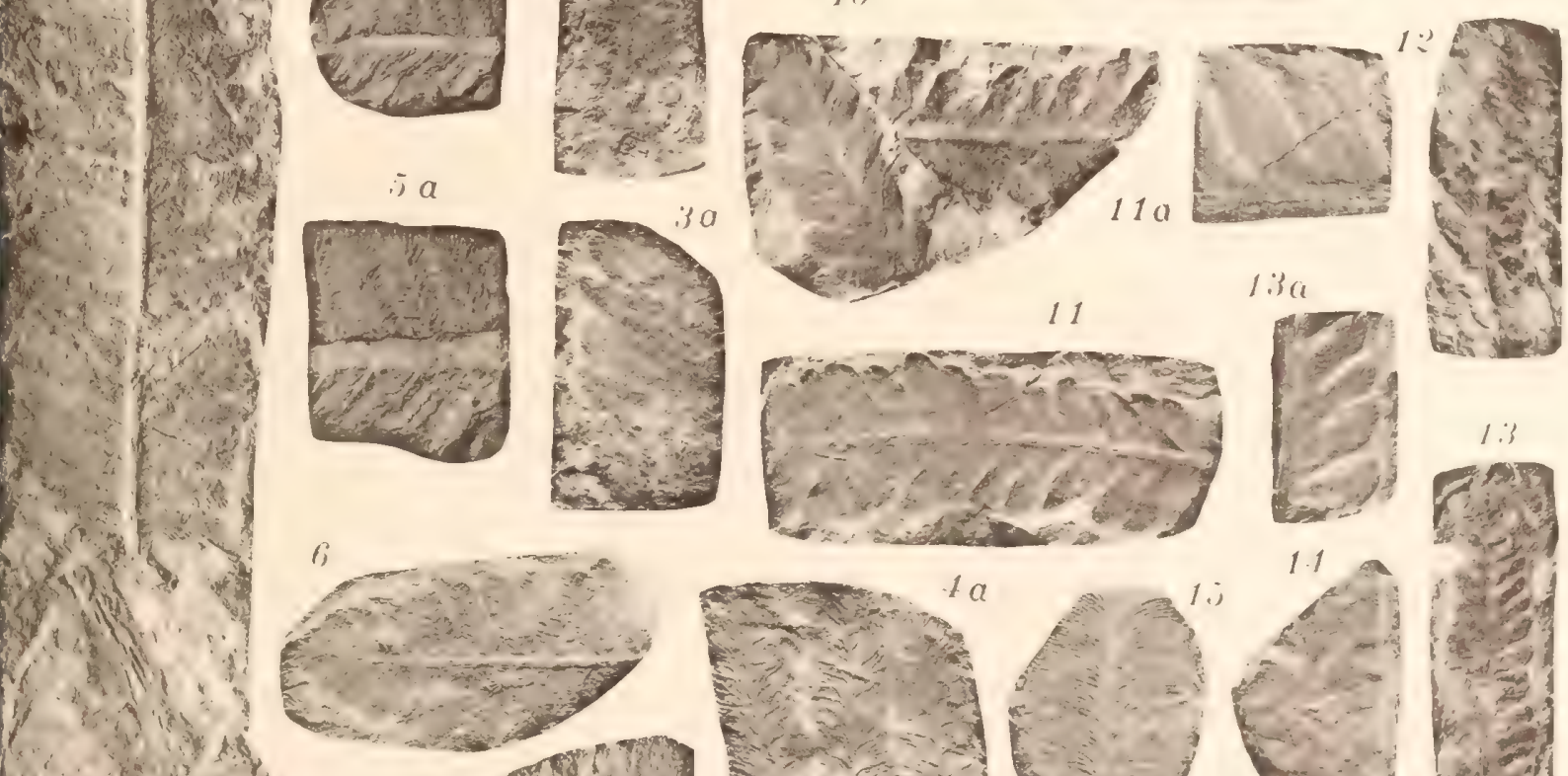

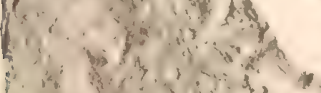
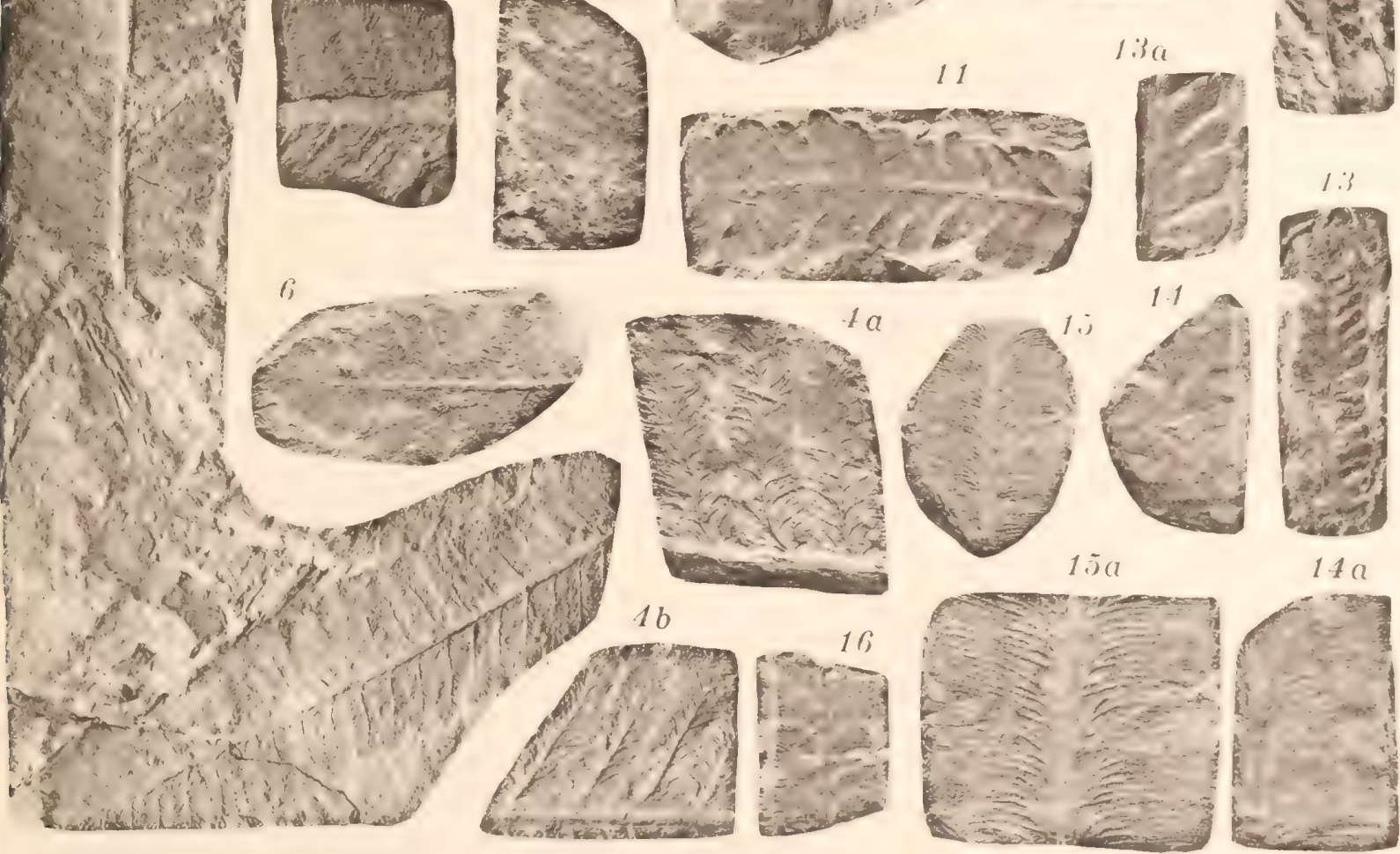

PLANCHE II 


\section{PLANGHE II.}

\section{EXPIICATION DES FIGURES.}

Fig. 1 à 3. - Cladophlebis (Todea) Rœsserti Presl (sp.). - Fragments de frondes. Hongay, mine de Carrère, au toit de la couche Chater.

Fig. 3 a. - Portion de l'échantillon fig. 3 , grossie deux fois.

Fig. 4. - Cladophlebis (Todea) Roesserti Presl (sp.). - Fragment de penne.

Mines de Hongaỹ : Hatou, découvert Nord, au mur de la grande couche.

Fig. 4a. - Portion du même échantillon, grossie deux fois.

FIG. 5, 6. - Gladophlebis (Todea) Roesserti Presl (sp.). - Fragments de frondes.

Mines de Hongay: Hatou, au toit de la grande couche.

FIG. 5a, 6a, 6b. - Portions des mêmes échantillons, grossies deux fois.

FIG. 7. - Cladophlebis (Todea) Rosserti Presl (sp.). - Fragment de fronde.

Hongaÿ, mine de Carrère, au toit de la couche Chater.

Fig. 7a - Portion du même échantillon, grossie deux fois. 
P1. II
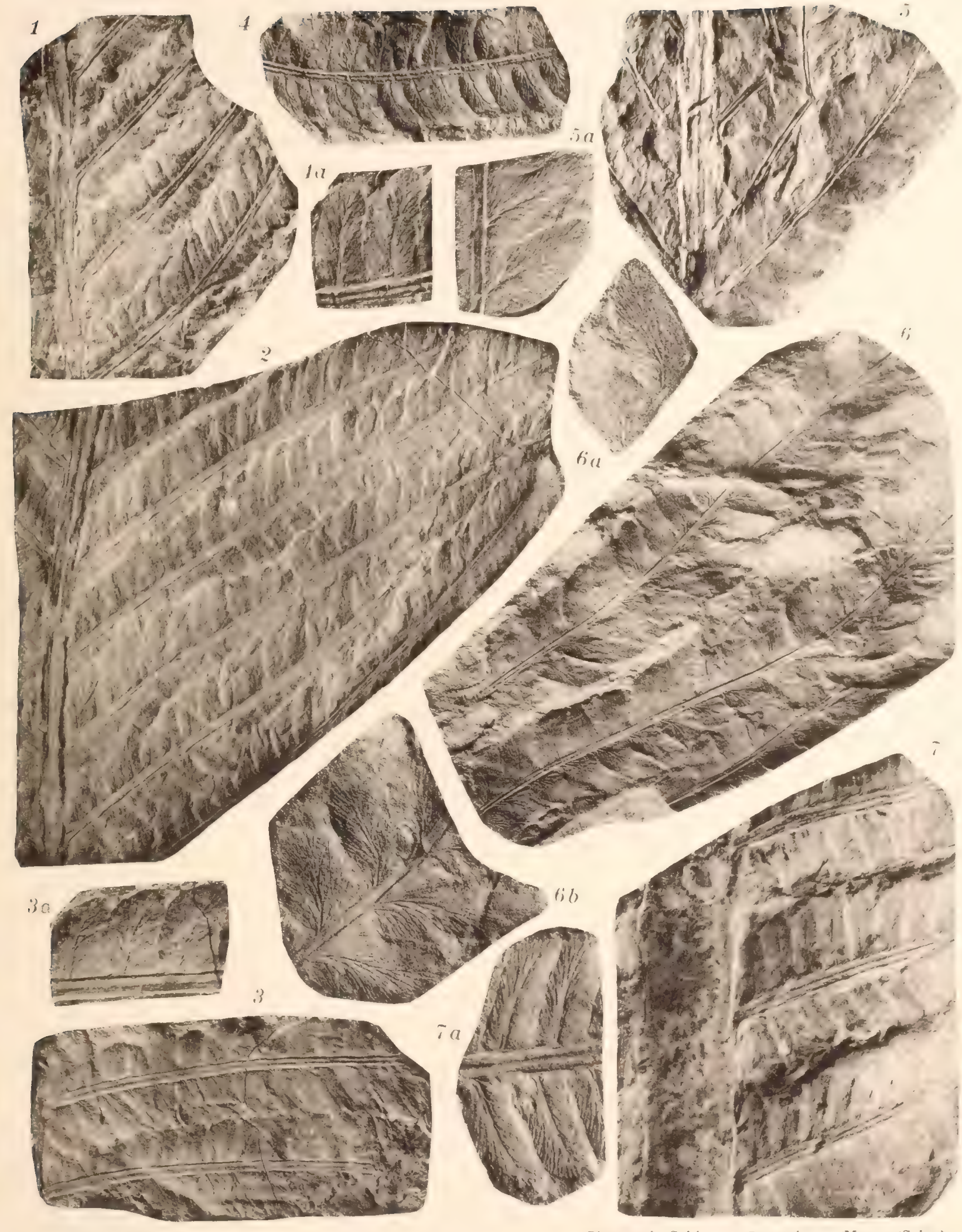

The
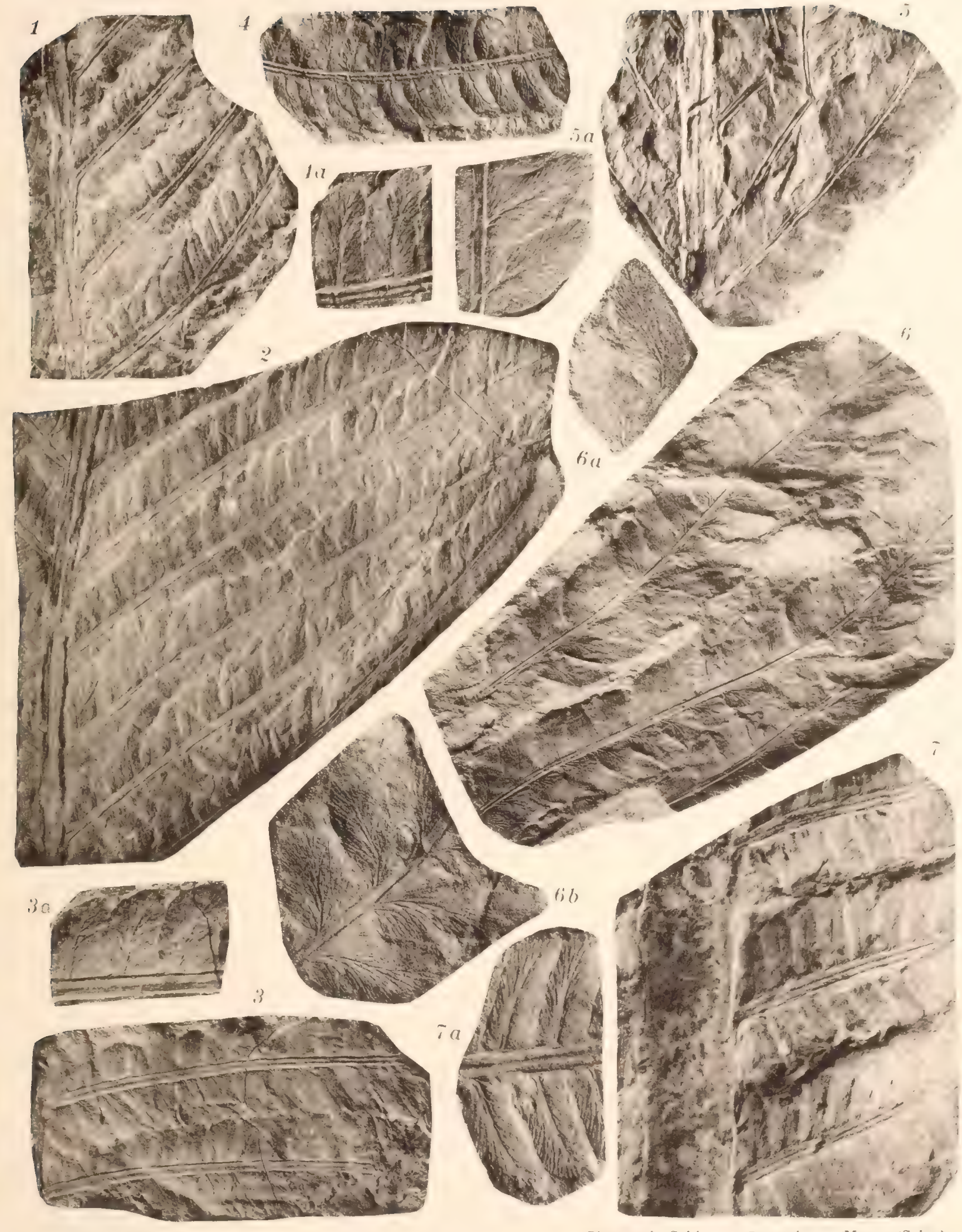

d) avertory (1)

Clichés Sohier.

Phototypie Sohier - Champigny-s/Marne (Seine) 

PLANCHE III 


\section{PLANCHE III.}

\section{EXPLICATION DES FIGURES.}

F1G. 1. - Cladophlebis (Todea) Rœsserti Presl (sp.). - Empreinte d'une fronde fertile. Kébao, puits Lanessan, travers-bancs Nord.

FIG. la, 1 b. - Portions du même échantillon, grossies deux fois.

Fıg. Ic, ld, le, lf - - Portions du même échantillon, grossies vingt-cinq fois, montrant les empreintes des sporanges.

Fig. 2. - Gladophlebis (Todea) Rœsserti Presl (sp.). - Empreinte d'un fragment de penne fertile.

Mines de Hongaÿ : Hatou, grande couche.

FIG. 2a. - Portion du même échantillon, grossie deux fois.

Fig. 3. - Cladophlebis (Todea) Rœesserti Presl (sp.). - Fragment de penne fertile. Hongaÿ, mine de Carrère, au mur de la couche Marmottan.

Fig. 3a. - Portion du même échantillon, grossie deux fois.

FIG. 3 b. - Sporanges du même échantillon, grossis vingt-cinq fois. 
Pl. III

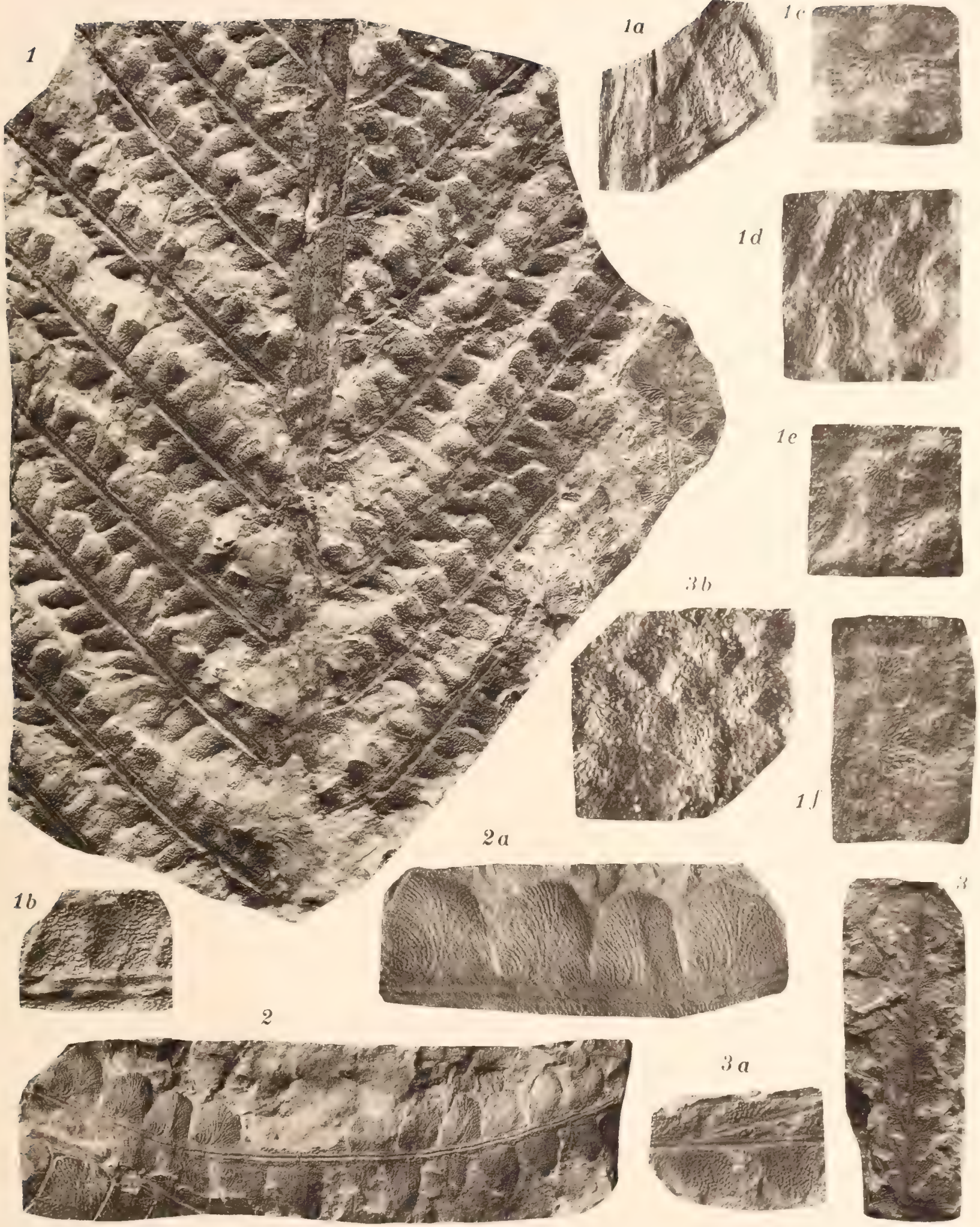





\section{PLANCHE IV}




\section{PLANCHE IV.}

\section{EXPLICATION DES FIGURES.}

Fig. 1. - Cladophlebis cf. lobifolia Philups (sp.). - Portion de fronde.

Kébao, puits Lanessan, étage de 120 mètres, travers-bancs Nord.

Fig. la, I b, lc. - Portions du même échantillon, grossies deux fois.

Fic. 2 à 4. - Gladophlebis nebbensis Brongniart (sp.). - Fragments de frondes. Hongaỹ, mine de Carrère, au toit de la couche Bavier.

Fıg. $3 \mathrm{a}, 4 \mathrm{a}, 4 \mathrm{~b}, 4 \mathrm{c}$.- Portions des échantillons fig. 3 et fig. 4, grossies deux fois. 

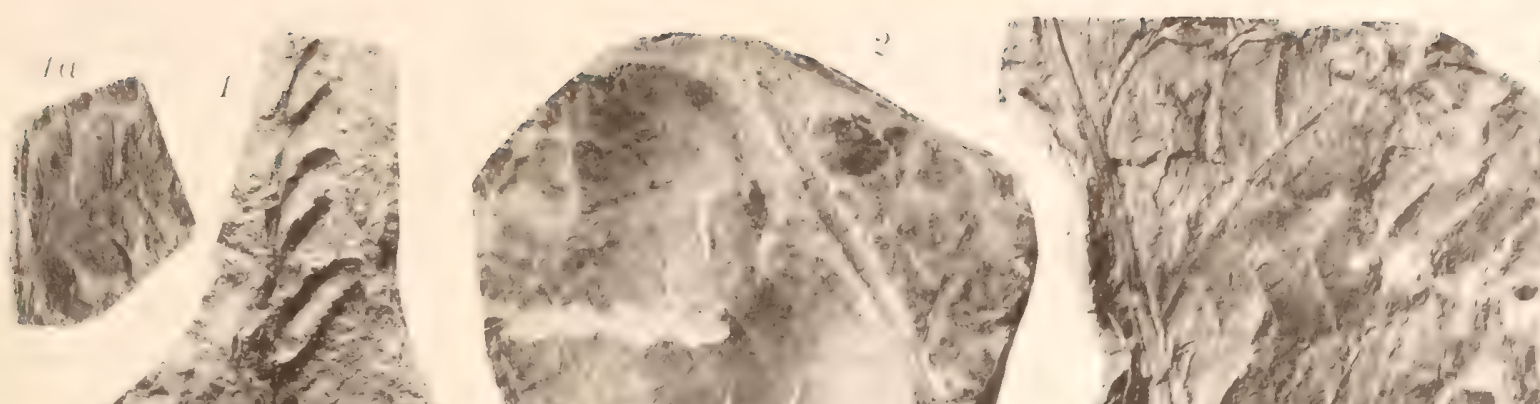

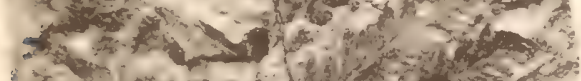

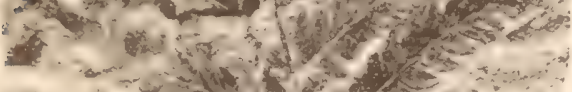

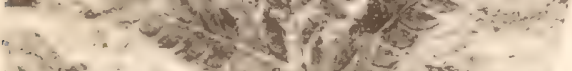

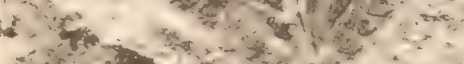

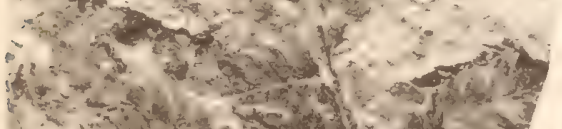

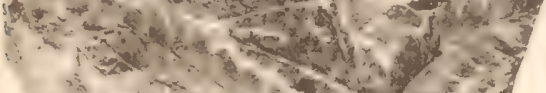

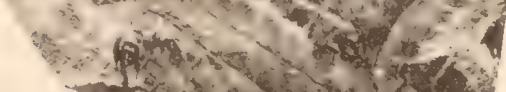
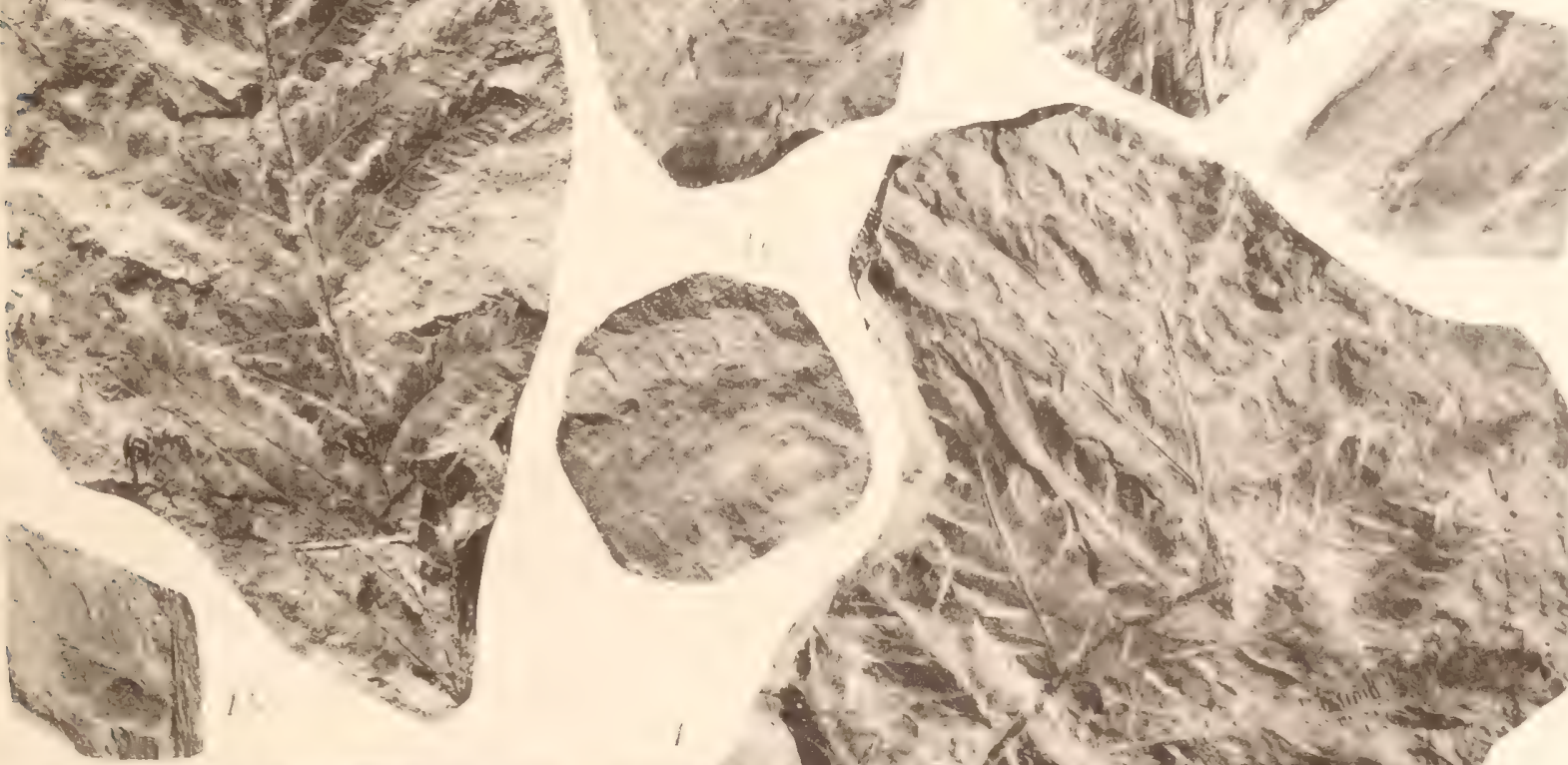

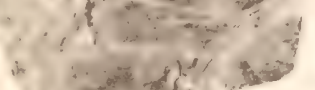
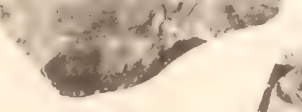
de $y+x^{2}+2$

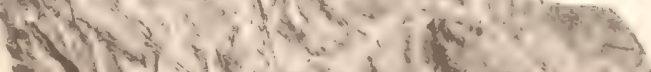

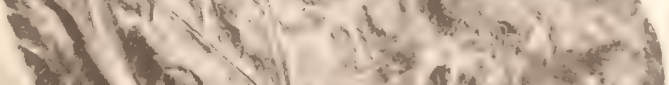

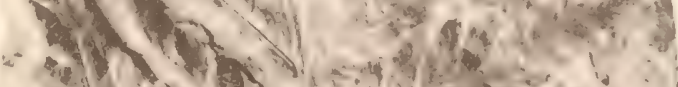

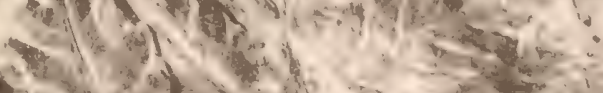

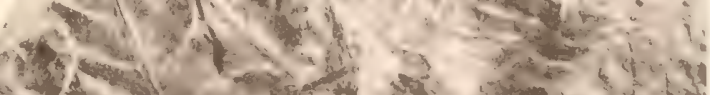

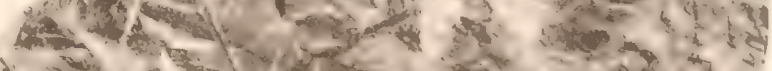

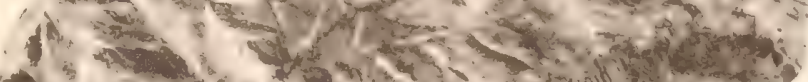

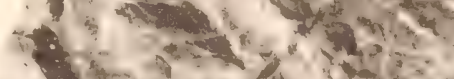

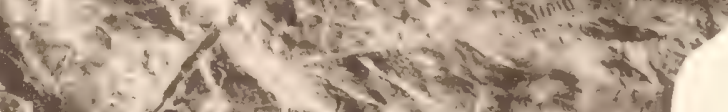

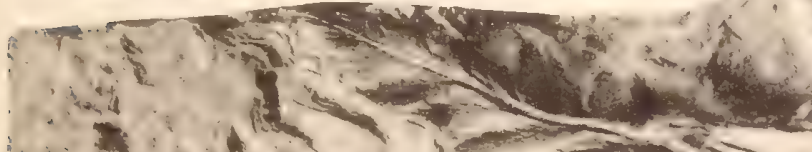

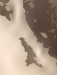

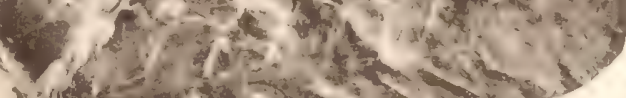

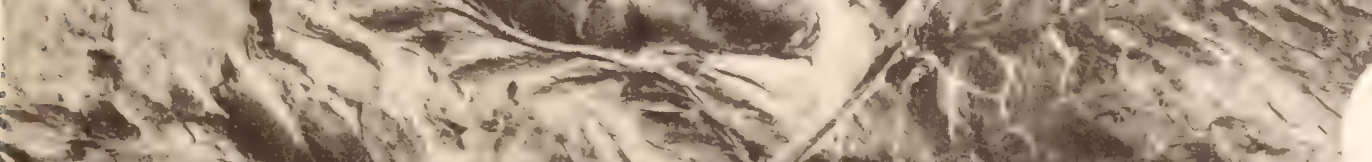

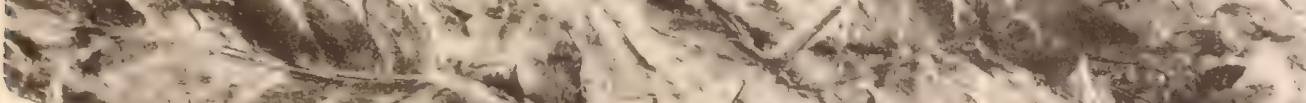

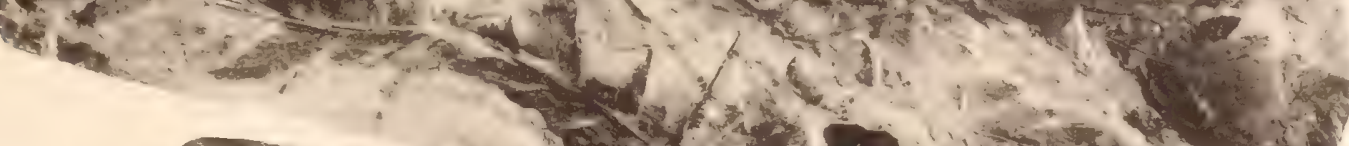
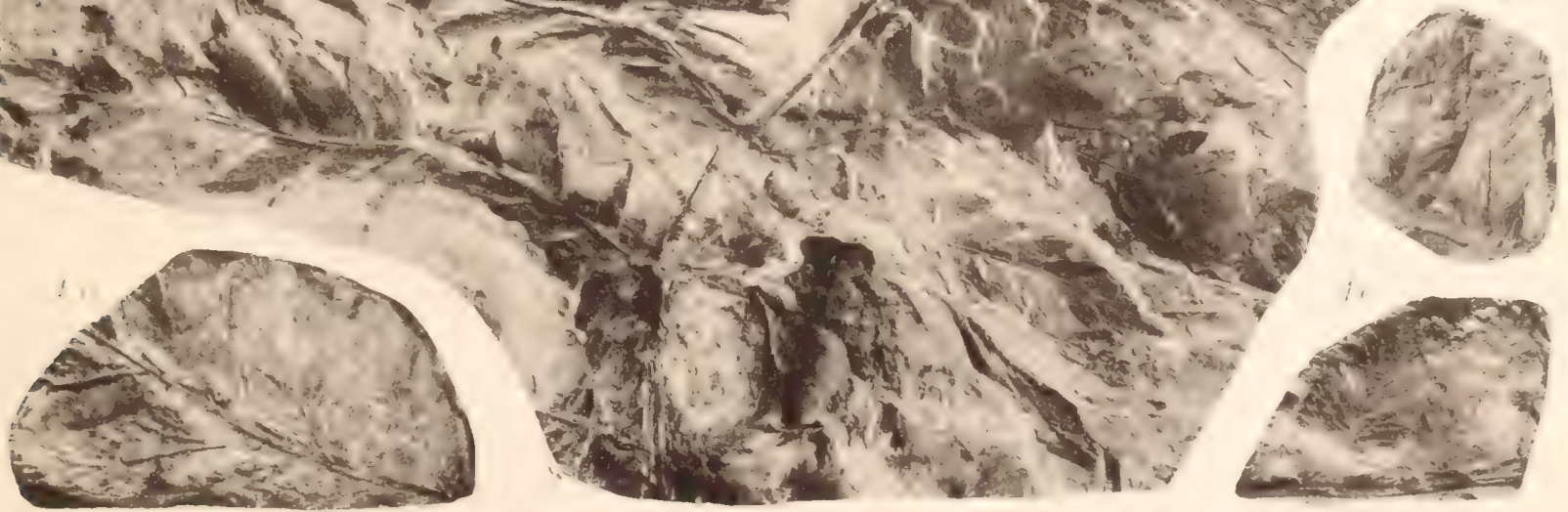



\section{PLANCHE V}

GiTES DE CHARBOX DU TOYKIV. 


\section{PLANGHE V.}

\section{EXPLICATION DES FIGURES.}

FIG. 1. - Cladophlebis Raciborskii n. sp. - Fragments de fron des.

Hongaÿ (collections de paléobotanique du Muséum d'histoire naturelle de Paris).

Fig. 1 a , I b, 1 c. - Portions du même échantillon, grossies deux fois. 

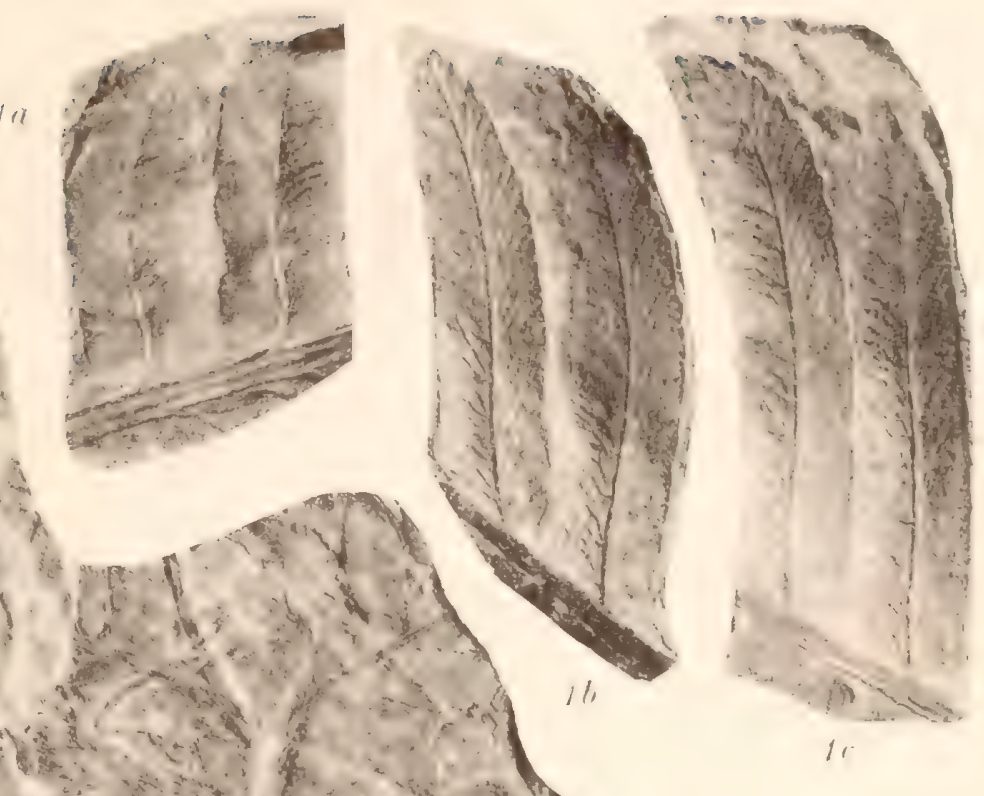

(1) 3 in

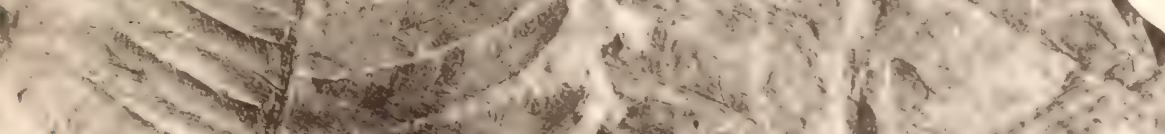

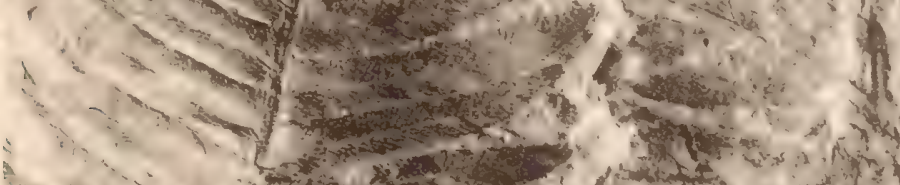

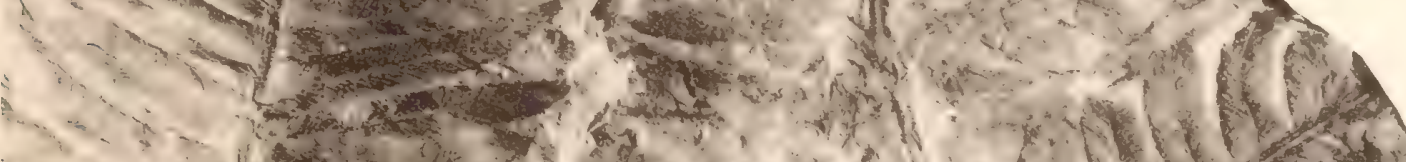

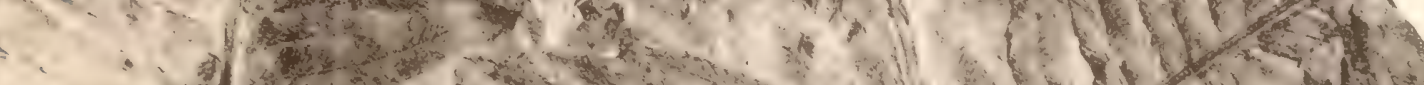

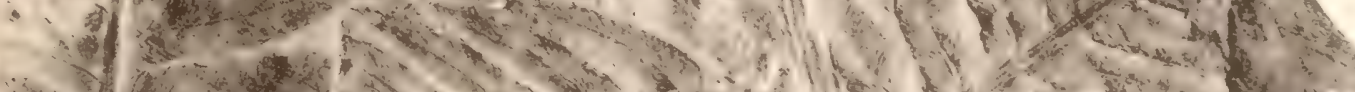

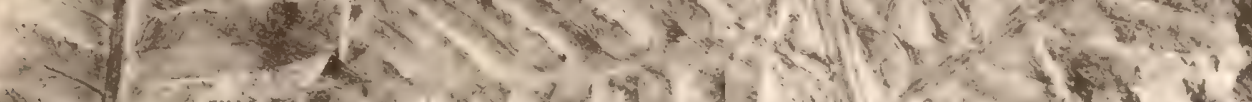

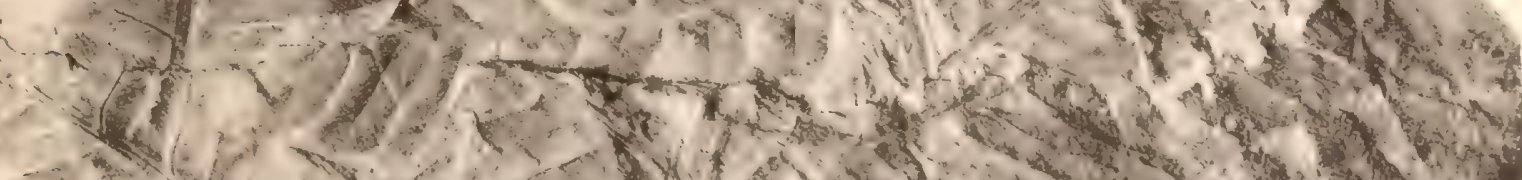

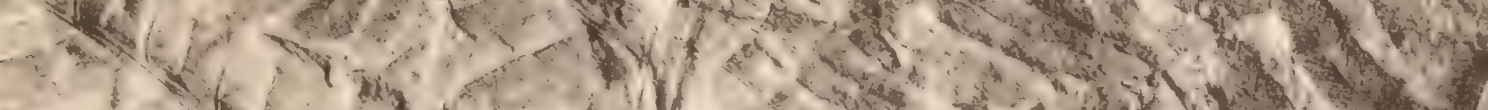
F. How

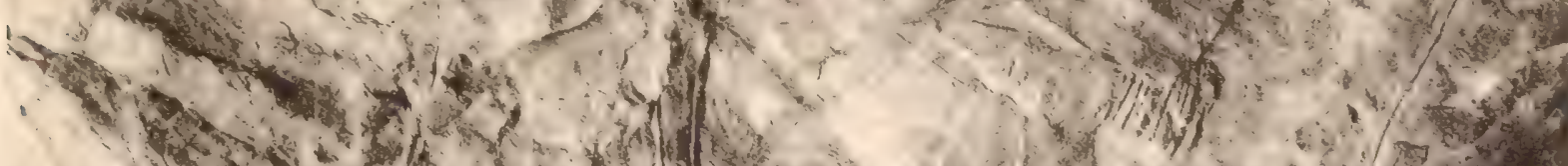

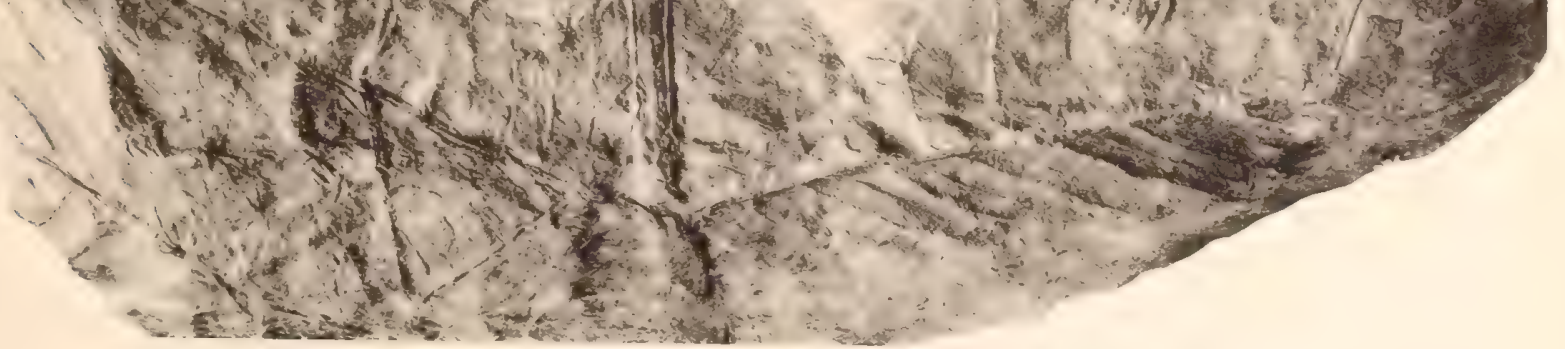



PLANCHE VI-VII 


\section{PLANCHE VI-VII.}

\section{EXPLICATION DES FIGURES.}

FIG. 1. - Gtenopteris Sarrani n, sp. - Poriion de fronde.

Kébao, à 20 mètres au toit de la couche principale de la galerie G; système inférieur.

Fig. 1 a. - Portion du même échantilton, grossie une fois et demie. 

(1) (1) 1, (2) 1.125 1. 45

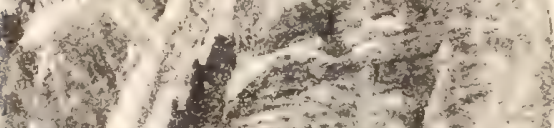

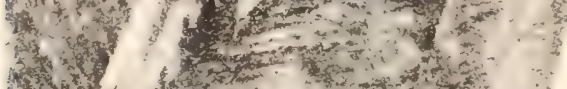

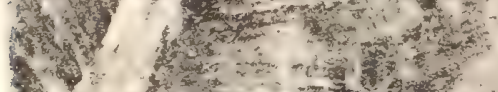
2. . . mo (1) artas? a.t. $(1,7)$

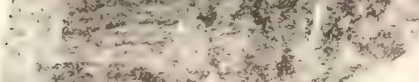

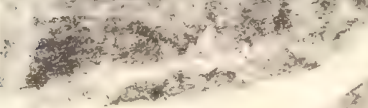

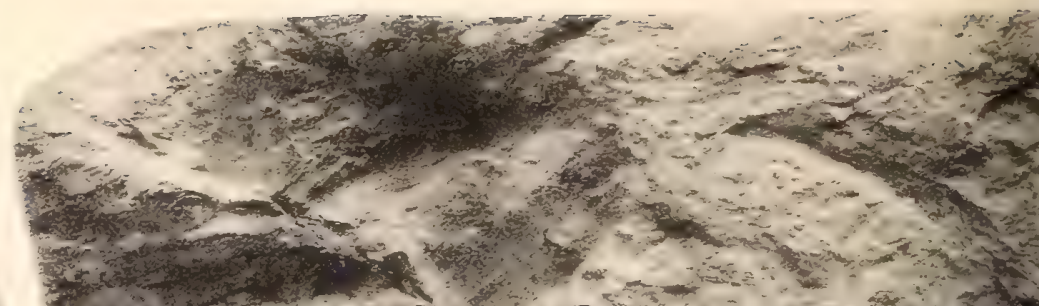

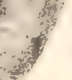

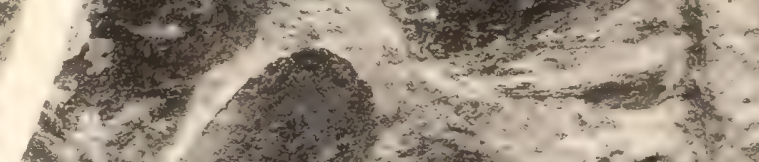

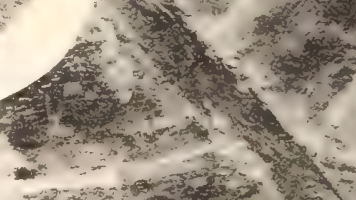
(1)

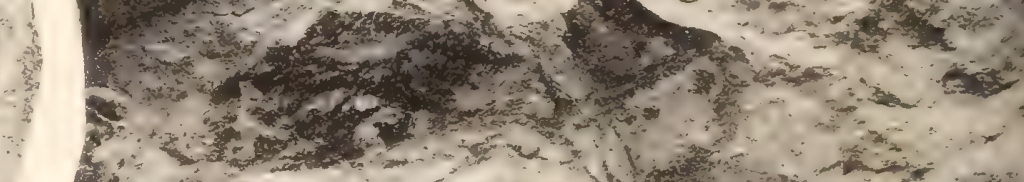

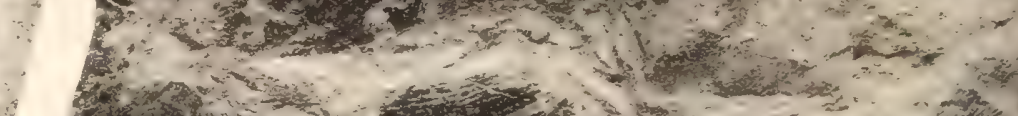

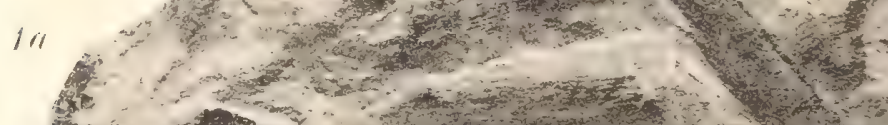

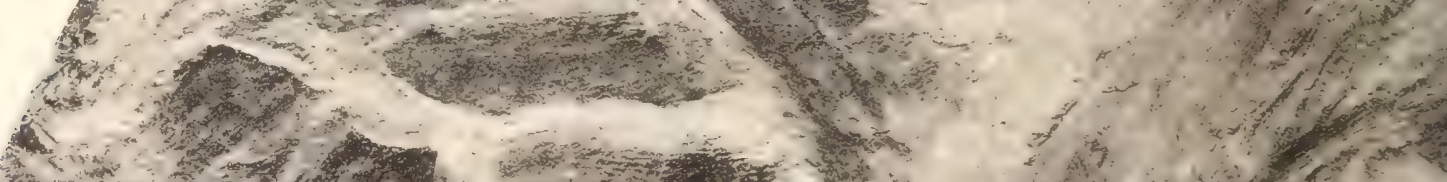

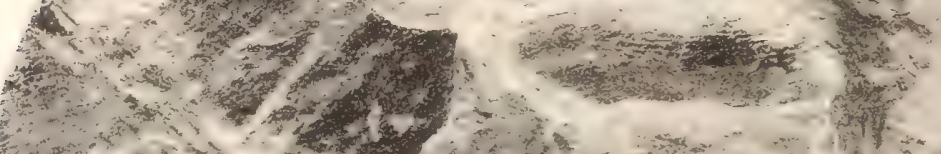

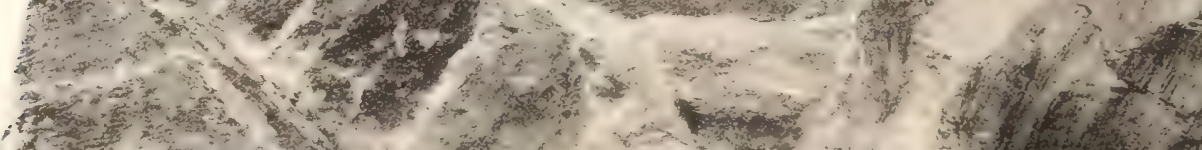

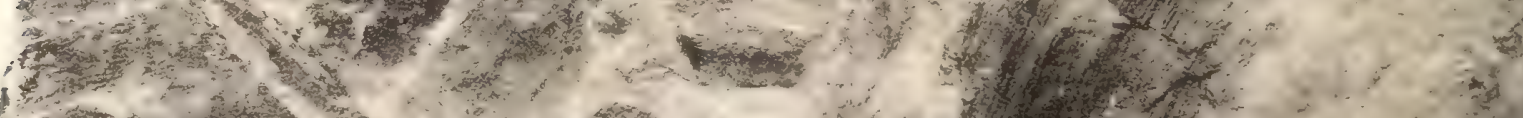

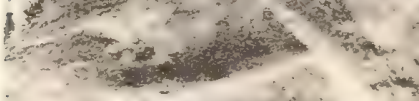

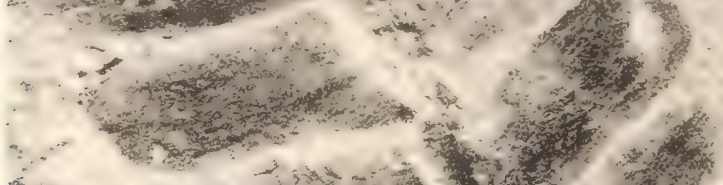

(1)

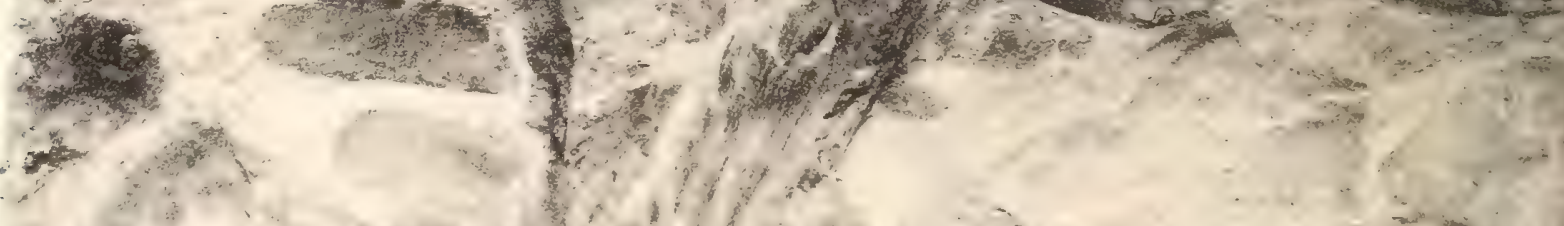

$\rightarrow-2+2$

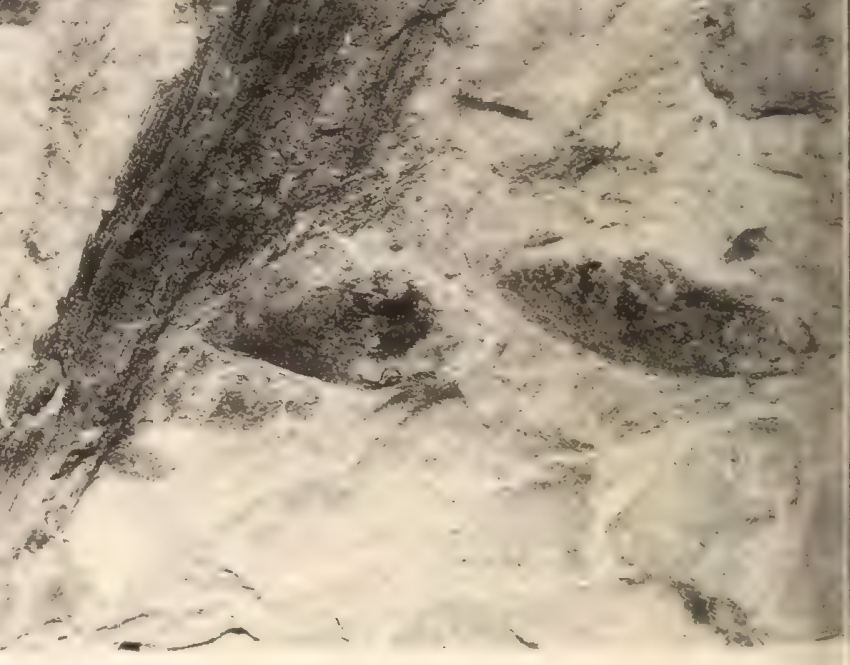



PLANCHE VIII 


\section{PLANCHE VIII.}

\section{EXPLICATION DES FIGURES.}

FIG. 1, 2. - Ctenopteris Sarrani n. sp. - Fragments de frondes.

Kébao, à 20 mètres au toit de la couche principale de la galerie G; système inférieur. 
I'1. VIII.

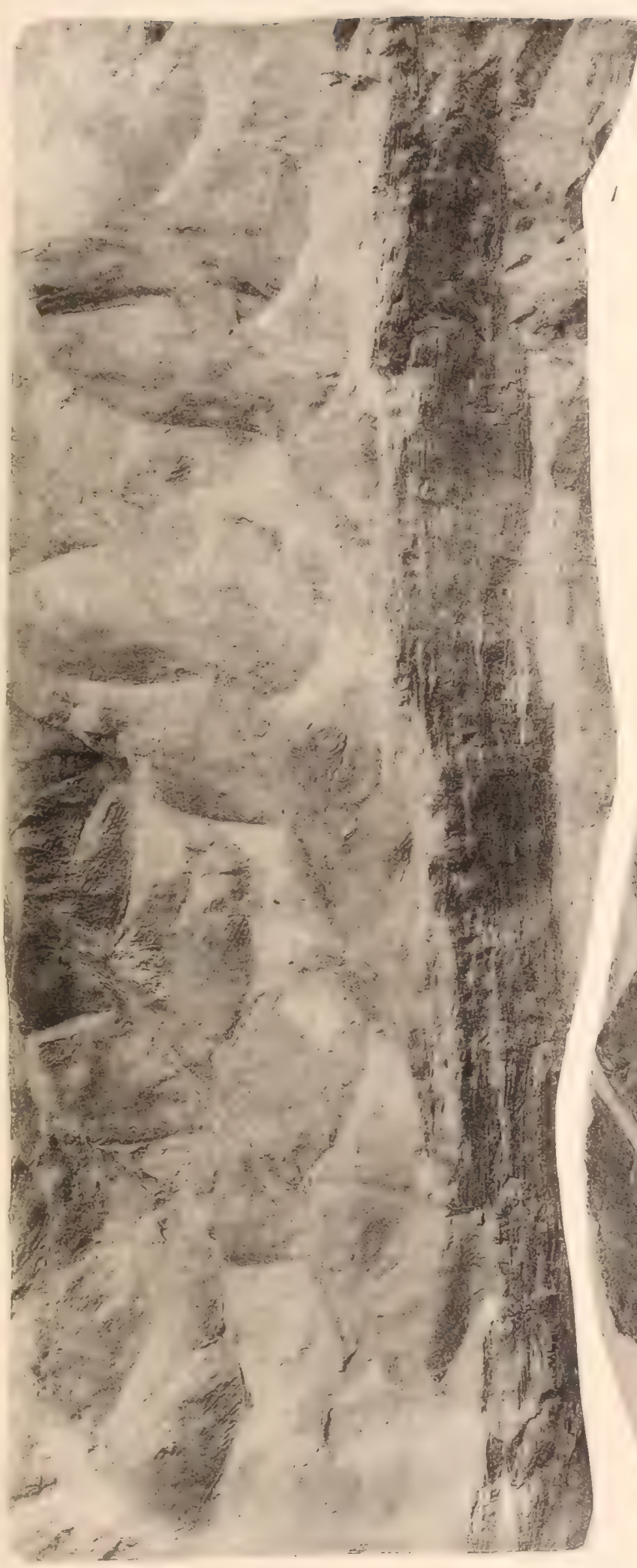

riliches sohion.

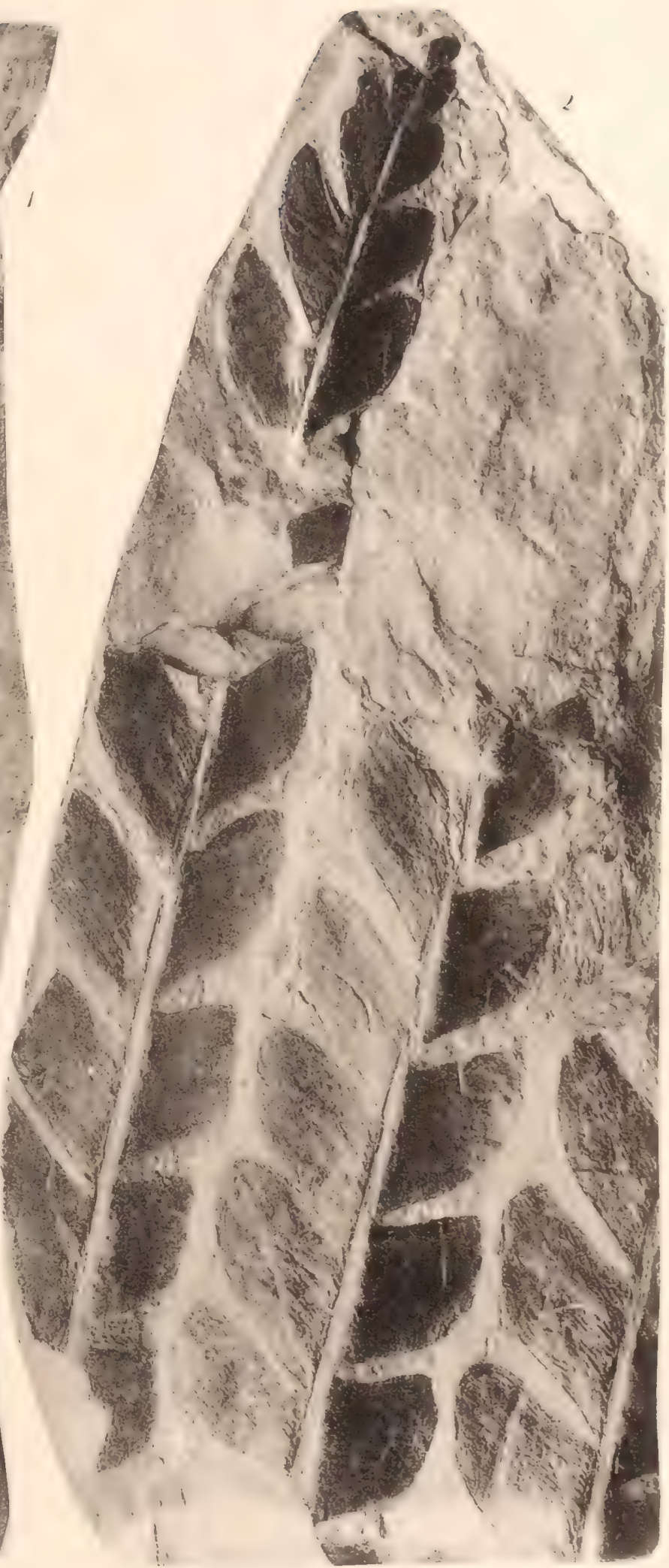

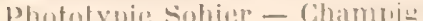


PLANCHE IX 


\section{PLANCHE IX.}

\section{FXPLICATION DES FIGURES.}

Fig. 1. - Danæopsis ef. Hughesi Feistmantel. - Fragment de penne.

Hongay̆, île du Sommet Buisson, galerie Jean.

Fig. 1 a. - Portion du même échantillon, grossie deux fois.

Fıc. 2. - Tæniopteris ensis OldHAY. - Fragment de fronde (figuré Annales des mines, 8 e série, t. II, pl. XII, fig. 2).

Île de Hongaỹ.

FIG. 2a. - Portion du même échantillon, grossie deux fois.

Fig. 3. - Tæniopteris cf. Mac Glellandi Oldham et Morris (sp.). - Fragment de fronde. Mines de Hongaÿ, découvert de Hatou.

Fig. 3 a. - Portion du mème échantillon, grossie deux fois.

Fig. 4. - Tæniopteris ef. Mac Glellandi Oldham et Morris (sp.). - Fragment de fronde (figuré Annales des mines, $8^{\mathrm{e}}$ série, t. II, pl. X, fig. 5 ). Lang-San.

Fig. 4a, 4 b. - Portions du même échantillon, grossies deux fois.

Fif. 5. - Tæmiopteris cf. Mac Glellandi Oldham et Morris (sp.). - Fragment de fronde.

Lang-San.

Fig. 6. - Tæniopteris (Marattia) Münsteri Gogppert. - Fragment de penne en partie fertile (figuré Bull. Soc. Géol. de France, $3^{\mathrm{e}}$ série, t. XIV, pl. XXIV, fig. 6).

Bassin de Hongaỹ.

Fig. 6a. - Portion du même échantillon, grossie deux fois.

Fig. 7. - Tæniopteris (Marattia) Münsteri Gorppent. - Fragment de penne fertile (figuré Bull. Soc. Géol. de France, t. XIV, pl. XXIV, fig. 7). Bassin de Hongä̈.

Fí. 8. - Tæniopteris (Marattia) Münsteri Goeppert. - Partie supérieure d'une penne fertile (figurée Bull. Soc. Géol. de krance, t. XIV, pl. XXIV, fig. 5).

Bassin de Hongaÿ.

Fig. 8 a. - Portion du même échantillon, grossie deux fois.

Fig. 8 b. - Portion du même échantilton, grossie quatre fois. 
P1. IX
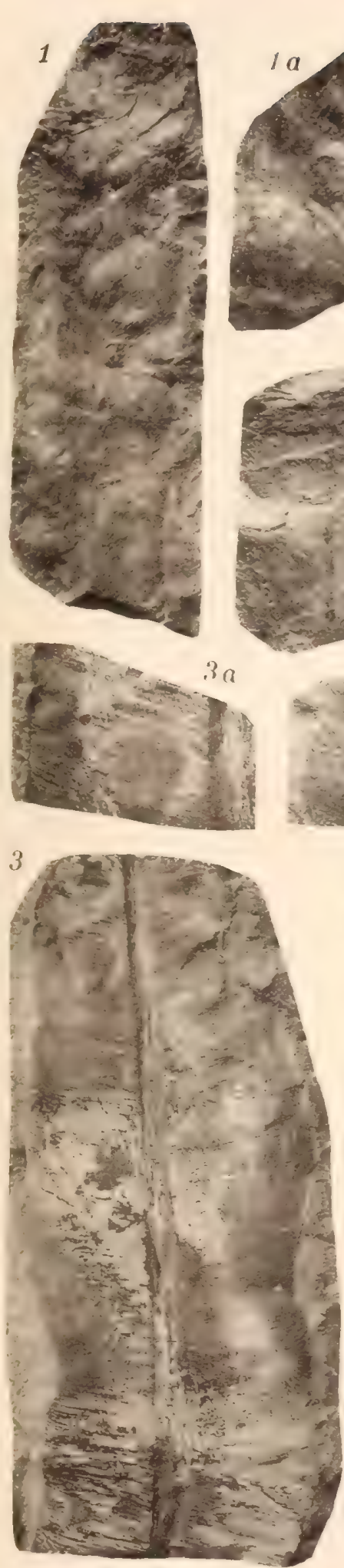

Clichis Sohier.
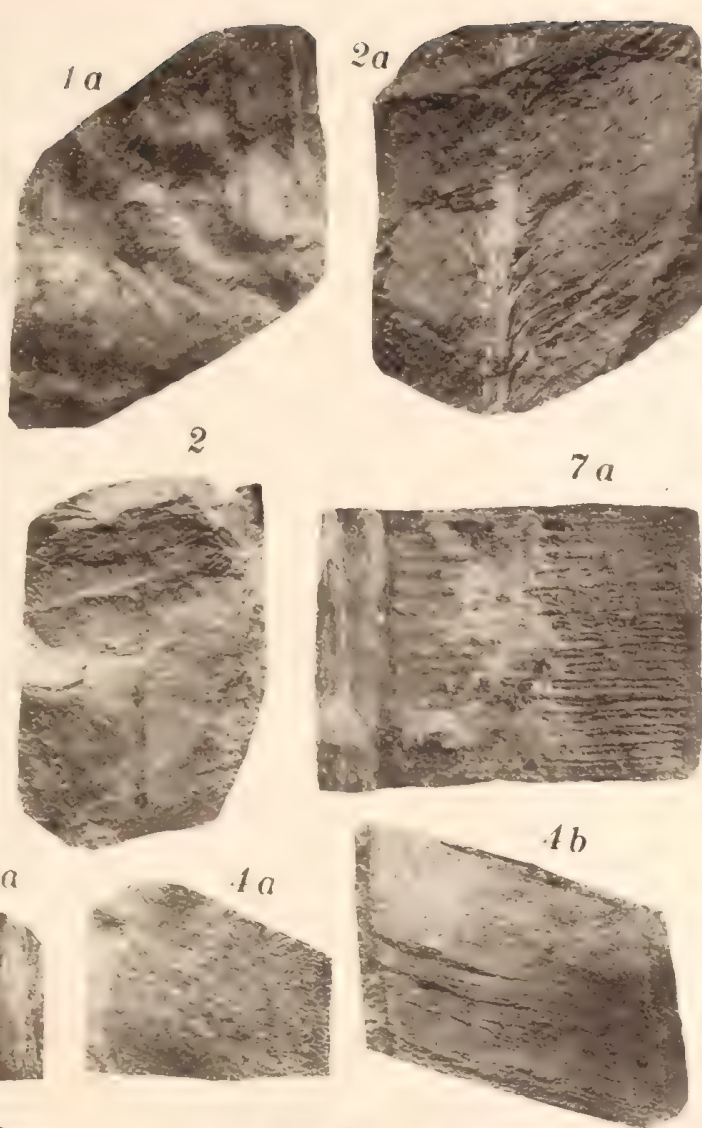
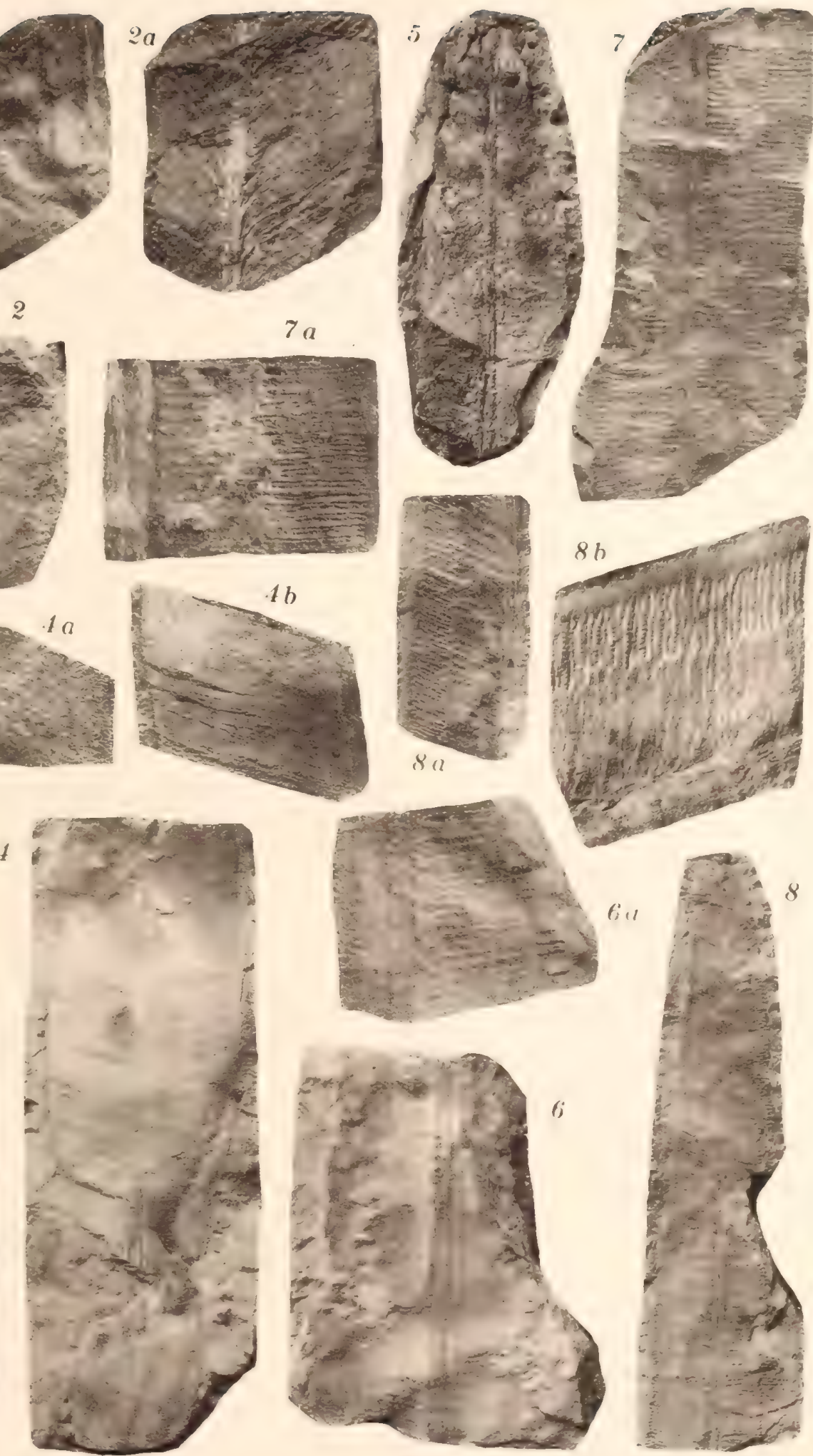

Phototypie Sohier - Champigny-s/Marne (Seine) 


\section{PLANCHE Y}




\section{PLANCHE X.}

\section{HXPLIGATION DES FIGURFS.}

FIG. 1. - Tæeniopteris Jourdyi ZeILler. - Sommet d'une fronde. Hongay, île du Sommet Buisson, galerie Jean.

FIG. I a. - Portion du même échantillon, grossie deur fois.

Vig. 2. - Tæniopteris Jourdyi Zenler. - Partie inférieure d'une fronde, brusquement contractée à la base.

Hongay̆, île du Sommei Buisson, galerie Jean.

Fig. 3. - Tæniopteris Jourdyi ZenLER. - Sommel d'une fronde.

Hongaÿ, mine de Carrère, au toit de la couche Marmotlan.

Fì. 3 a. - Portion du même échantillon, grossie deus fois.

Fig. 4. - Tæniopteris Jourdyi Zeiller. - Fragment de fronde (figuré Bull. Soc. Géol. de France, $3^{\mathrm{e}}$ série, t. XIV, pl. XXV, fig. 2 ).

Bassin de Hongày.

Frg. 4a. - Portion du même échantillon, grossie deux fois.

Fig. 5. - Treniopteris Jourdyi ZerLler. - Fragment de fronde.

Hongaÿ, vallée orientale de l'OEuf, galerie Léonice.

Fic. 5 a. - Portion du même échantillon, grossie deux fois.

FIf. 6. - Tæniopteris Jourdyi ZeILler. - Fragment de fronde. Bassin de Hongay.

Fic. 6a. - Portion du même échantillon, grossie deux fois. 
P1. X
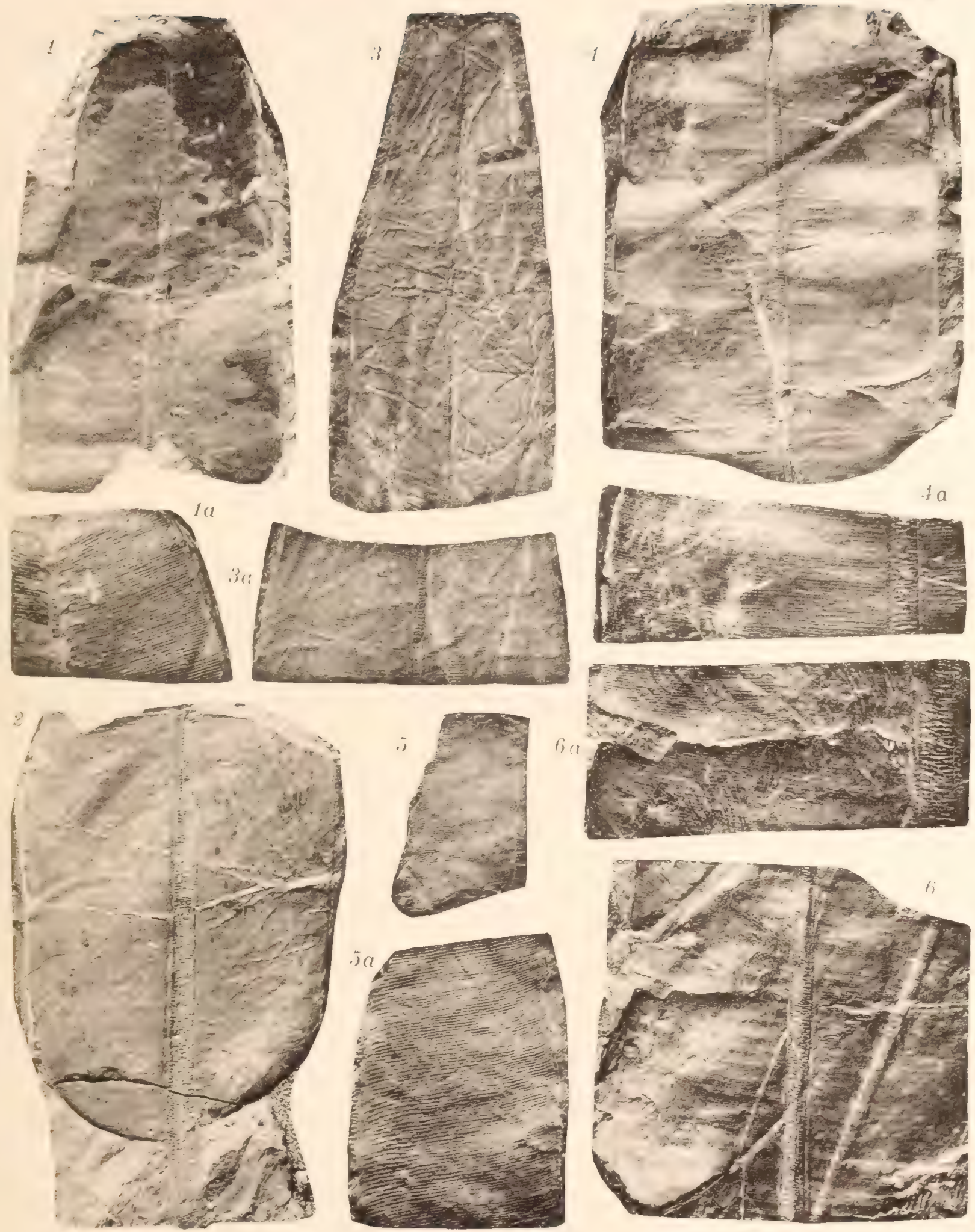

Clichés Sohier.

Phototypie Sohier - Champigny-s/Marne (Seine) 


\section{PLANGHE XI}

3. 


\title{
PIANCHE XI.
}

\author{
LEPICICTION IOES FIGURLSS
}

Fig. 1. - Tæniopteris Jourdyi ZEllLer. - Portion de fronde, à limber relativement étroit et décurrent le tong du pétiole.

Kébao, système supérieur, couche $\mathrm{n}^{\circ} 2$, galerie $\mathrm{M}$.

FIf. 2. - Tæniopteris Jourdyi ZEILLEn. - Partie inféricure d'une fronde, contractée à la base.

Kébao, système supérieur, couche $\mathrm{n}^{\circ} 2$, galerie $\mathrm{M}$.

Fig. 3. - Tæniopteris Jourdyi ZEILler. - Portion de fronde, à limbe très large, dricurrent le long du rachis.

Hongaj, mine de Carrère, au toit de la couche Marmottan.

Fif. 4. - Tæniopteris Jourdyi ZenLER. - Groupe de frondes partant d'un pied commun, auquel s'attachent également des racines; ces frondes sont réduites à leurs pétioles, à l'exception de l'une d'entre elles (sous le' chiffre 4) qui montre encore un fragment de la base du limbe.

Kúba, système supérieur, couche $n^{\circ} 2$, galerie M. 
P1. X?
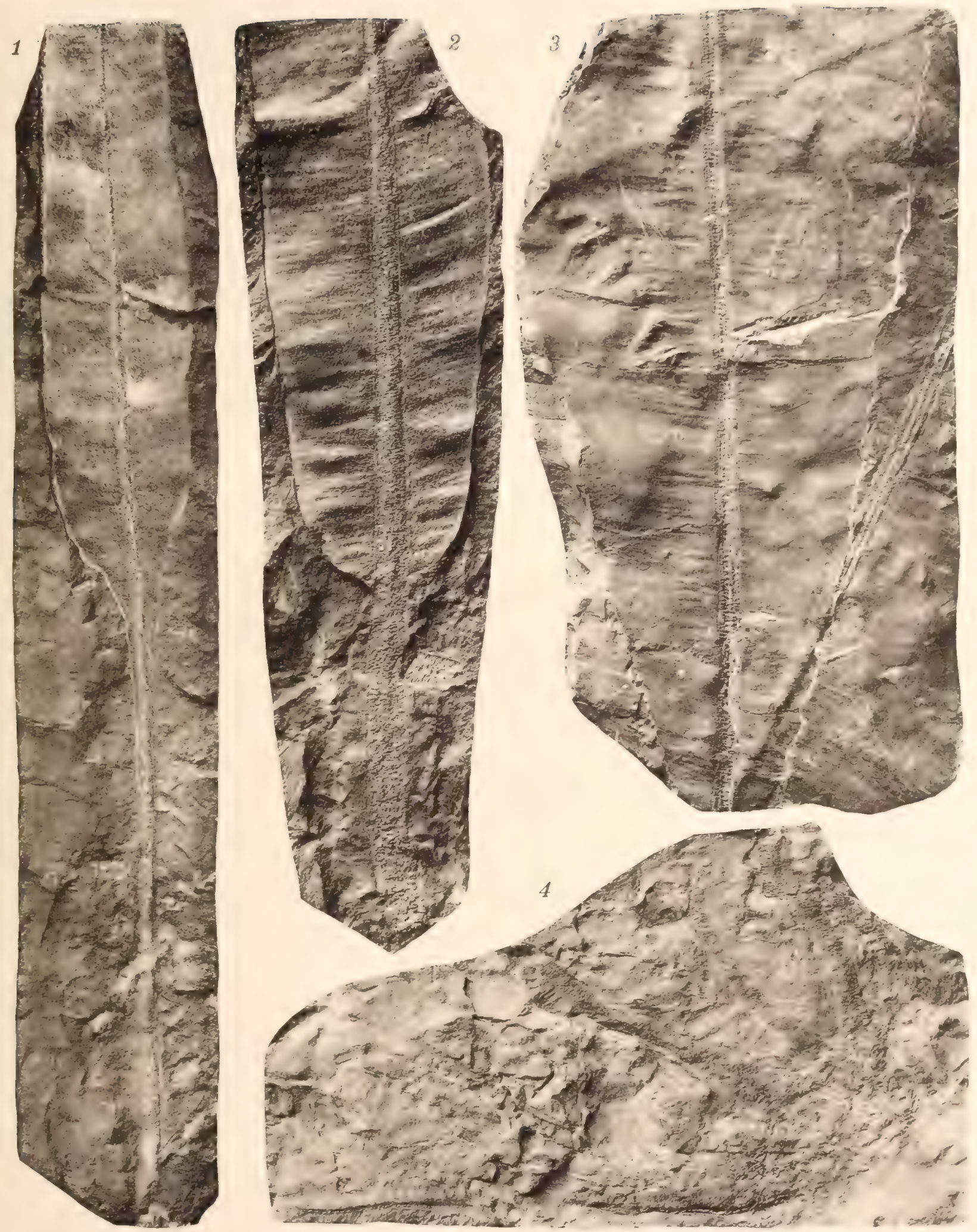

Clichés Sohier.

Phototypie Sohier - Champigny-s/Marne (Seine) 

PLANCHE XII 


\section{PLANCHE XII.}

\section{EXPLICATION DES FJGURES.}

Fig. 1 a. - Tæniopteris Jourdyi ZeILLer. - Fronde complete, accidentellement contractée à son sommet en une longue pointe aiguë; réduite à moitié de la grandeur naturelle.

Kébao, système supérieur, couche $\mathrm{n}^{\circ} 2$, galerie M.

FIG. 1 et $1^{\prime}$. - Base et sommet de la même fronde, grandeur naturelle.

Fig. 2 it 4. - Tæniopteris Jourdyi ZeILlER. - Sommets de frondes, montrant les variations de formes d'un échantillon à l'autre.

Kébao, système supérieur, couche $n^{\circ} 2$, galerie $\mathbf{M}$.

Fig. 5. - Empreinte d'attribution incertaine, représentant peut-être une fronde fertite de Teniopteris, à fructifications submarginales, imparfaitement développée.

Kébao, système supérieur, couche $\mathrm{n}^{\circ} 2$, galerie $\mathrm{M}$.

Fig. 6 et 7. - Tæniopteris Jourdyi ZeILler. - Bases de frondes à limbe plus ou moins longuement décurrent.

Kébao, système supérieur, couche $\mathrm{n}^{\circ} 2$, galerie M.

Fig. 8. - Tæniopteris Jourdyi Zeillek. - Portions de frondes à limbe accidentellement incisé et divisé en segments plus ou moins réguliers.

Mines de Hongaÿ, découvert de Hatou. 


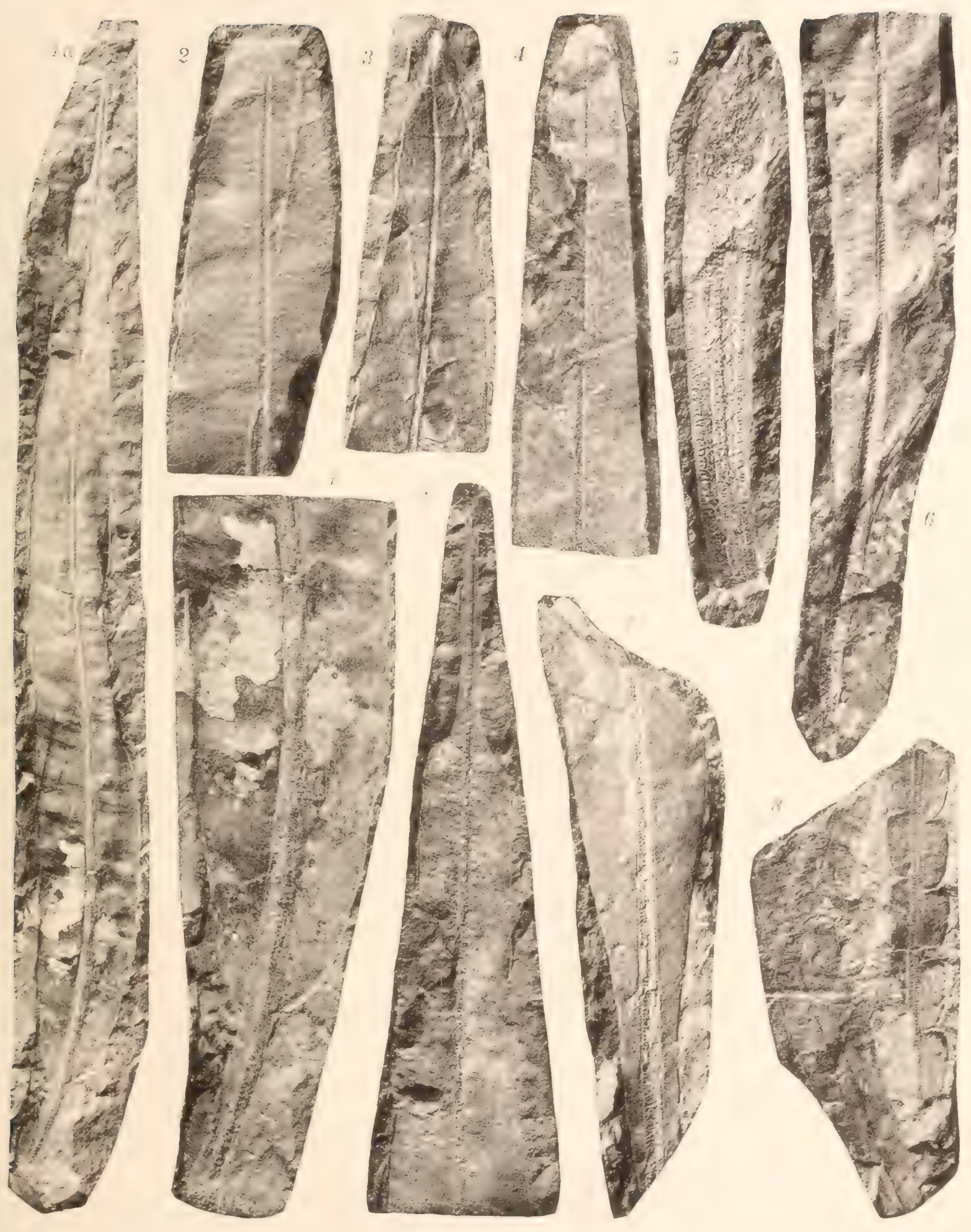

Clichés Sohier. 



\section{PLANCHE XIII}




\title{
PLANCHE XIII.
}

\author{
EAPIICATION DES FIGLRES.
}

FiG. 1. - Tæniopteris Jourdyi ZEILLER. - Fronde très étroite.

Vines de Hongaỹ : Hatou, au toit de la grande couche.

Fig. 2 à 4. - Tæniopteris Jourdyi Zeiller. - Fragments de frondes à limbe plus ou moins étroit.

Vines de Hongaỹ : Hatou, grande couche.

FIr. 3 a, 3 b et 4 a. - Portions des rechantillons fig. 3 et 4 , grossies deux fois.

Fig. 5. - Tæniopteris Jourdyi Zeiller. - Fragment de fronde à limbe très firoil. Vines de Hongaÿ: Gia-ham, près Hatou.

Fig. 5 a. - Pottion du même échantillon, grossie deux fois.

Fig. 6 et 7. - Tæniopteris spatulata MaG Cleluand. - Frondes incomplites. Hongay, rivière des Mines, rive droite, première vallée.

Fis, 8 et 9. - Tæniopteris spatulata MaC Clelland. - Parties inférieures do frondes. Mines de Hongaỹ : Nagotna.

Fıg. 8a. - Portion de l'échantillon fig. 8, grossie trois fois.

Fici, 10. - Tæniopteris spatulata Mac Gueldand. - Fronde presque complete. Hongaỹ, mine de Carrère, au toit de la couche Bavier.

Fro. 11 a. - Tæniopteris spatulata Mac Clelland. - Portion de fronde, grosie lrois fois.

Mines de Hongaÿ : Nagotna.

Firi. 12. - Taniopteris apatulata Mac Clelland. - Fragments de fiondes. Hongay, rivière des lines, rive droite, première vallír. 
PJ. XiII
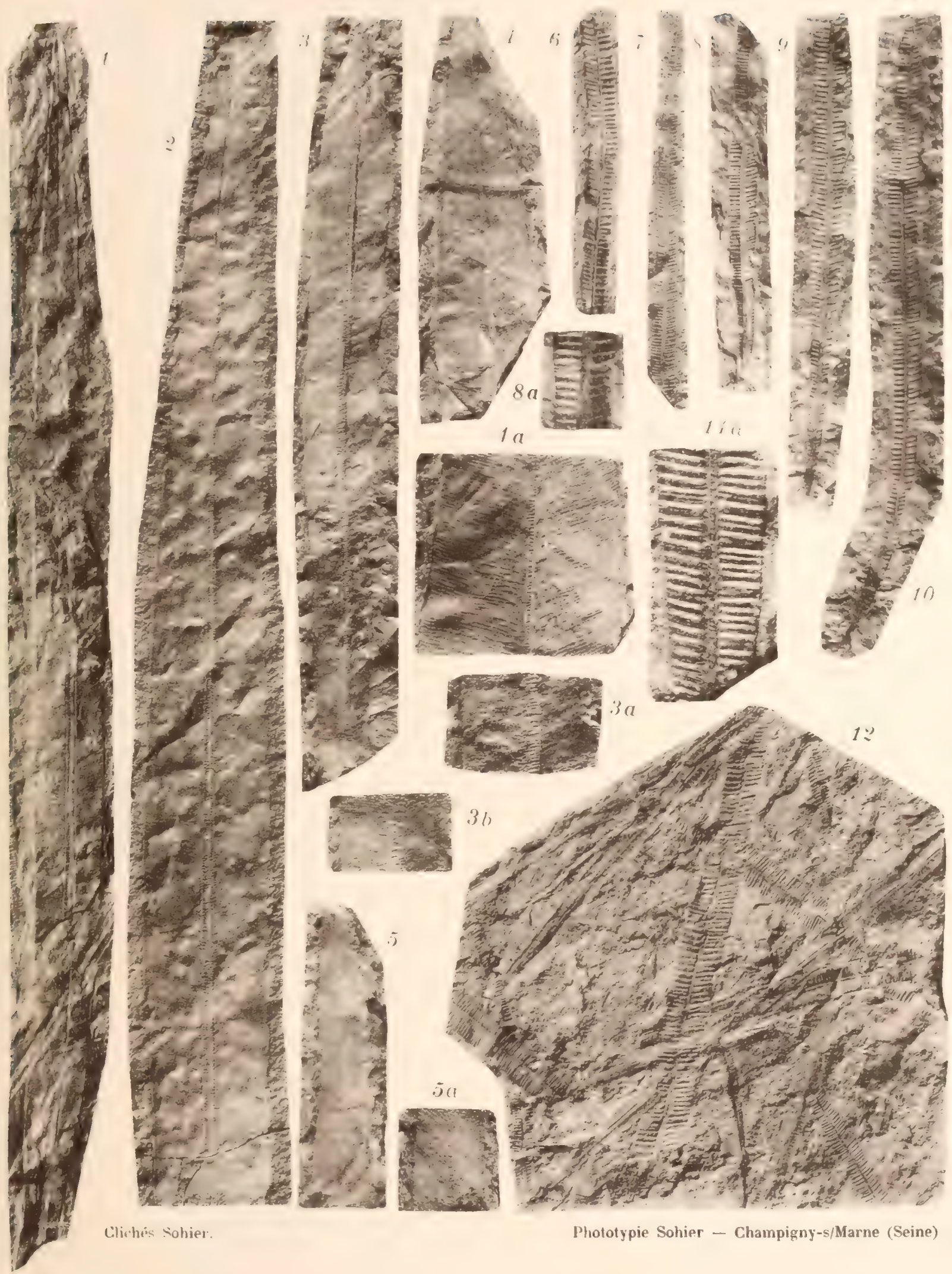

Phototypie Sohier - Champigny-s/Marne (Seine) 



\section{PLANCHE XIV}




\section{PLANCHE XIV.}

EXPLICATION DES FIGURES.

FIG. 1 à 3.-- Tæniopteris virgulata n. sp. - Portions de frondes. Kébao, couche G. 


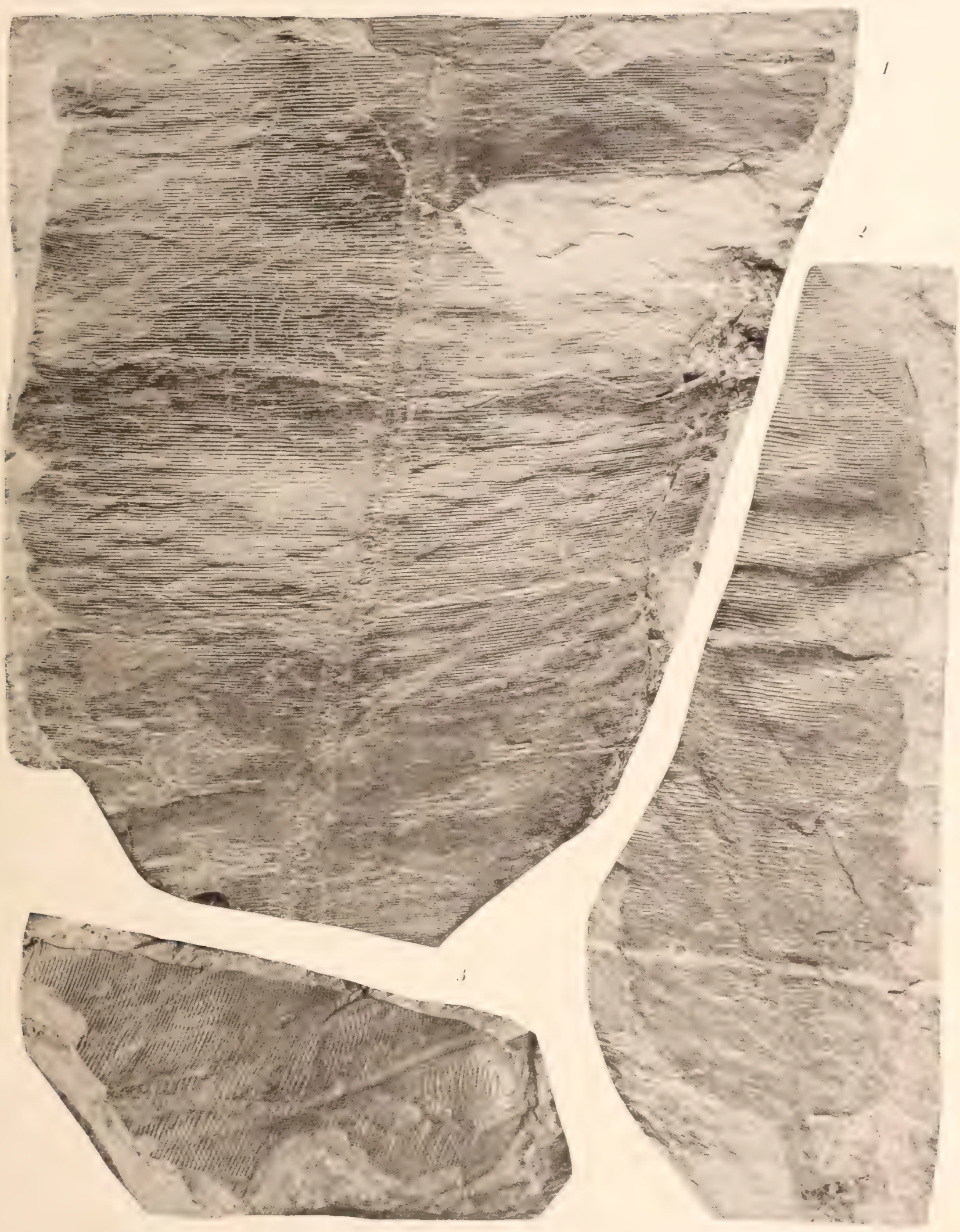

Clichés suhiet

Phototypie Sohior - Champigny s/Mane seine 



\section{PLANCHE XV}




\section{PLANCHE XV.}

\section{EXPLICATION DES FIGURES.}

FIG. 1. - Tæniopteris nilssonioides n. sp. - Portions de frondes. Mines de Hongaÿ, découvert de Hatou.

Fig. 2. - Tæniopteris nilssonioides n. sp. - Frondes de petite taille, complètes. Kébao, mine Rémaury, couche Q.

Fig. 2 a. - Portion du même échantillon (fragment de fronde de l'angle supérieur de gauche), grossie deux fois.

Fig. 3. - Tæniopteris nilssonioides n. sp. - Fragment de fronde.

Kébao, puits Lanessan.

FIG. 4. - Tæniopteris nilssonioides n. sp. - Fragment de fronde.

Kébao, système supérieur, couche $\mathbf{n}^{\circ}$ 2, galerie $\mathbf{M}$.

Fig. 4 a. - Portion du même échantillon, grossie deux fois. 
PI. XV

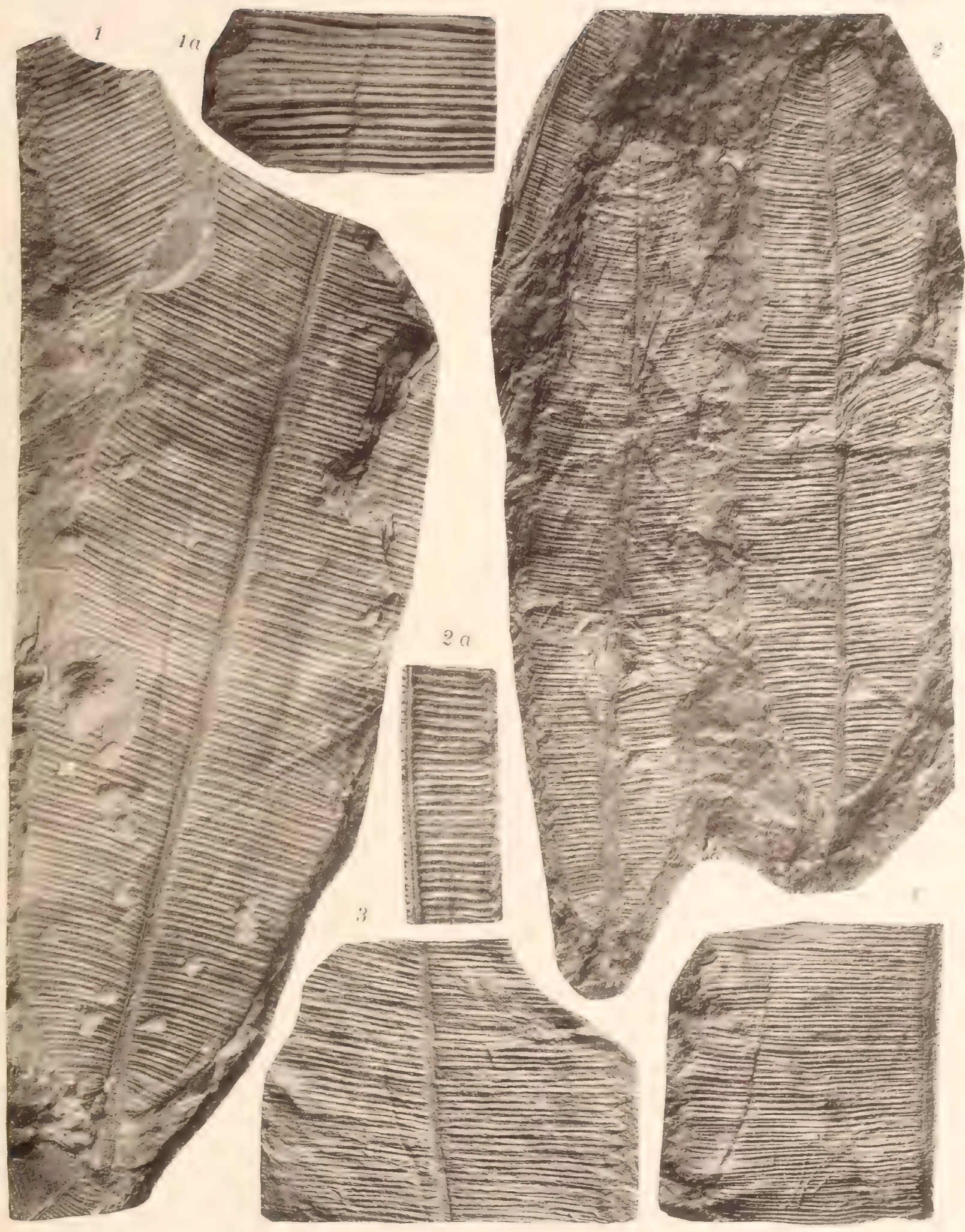

Clichés Sohier.

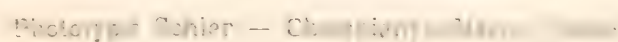





\section{PLANCHE XVI}




\section{PLANCHE XVI.}

\section{EXPLICATION DES FIGURES.}

Fig. 1. - Palæovittaria Kurzi Feistmantru. - Fragment de fronde (contre-empreinte de l'échantillon figuré Annales des Mines, $8^{\mathrm{e}}$ série, t. II, pl. XI, fig. 3). Kébao.

Fig. 1 a. - Portion du même échantillon, grossie deux fois.

FIg. 2. - Glossopteris indica Schimper. - Frondes plus ou moins incomplètes. Kébao, puits Lanessan.

Fig. 2 a. - Portion du même échantitlon, grossie deux fois.

Fig. 3 et 4. - Glossopteris indica Schimper. - Feuilles écailleuses. Mines de Hongaÿ : Gia-Ham, près Hatou.

Fig. 3 a. - Portion de l'échantillon fig. 3 , grossie deux fois.

Fig. 5. - Glossopteris indica Schimper. - Portion de feuille écailleuse.

Mines de Hongaÿ, découvert de Hatou (collections de géologie du Muséum d'histoire naturelle de Paris). 

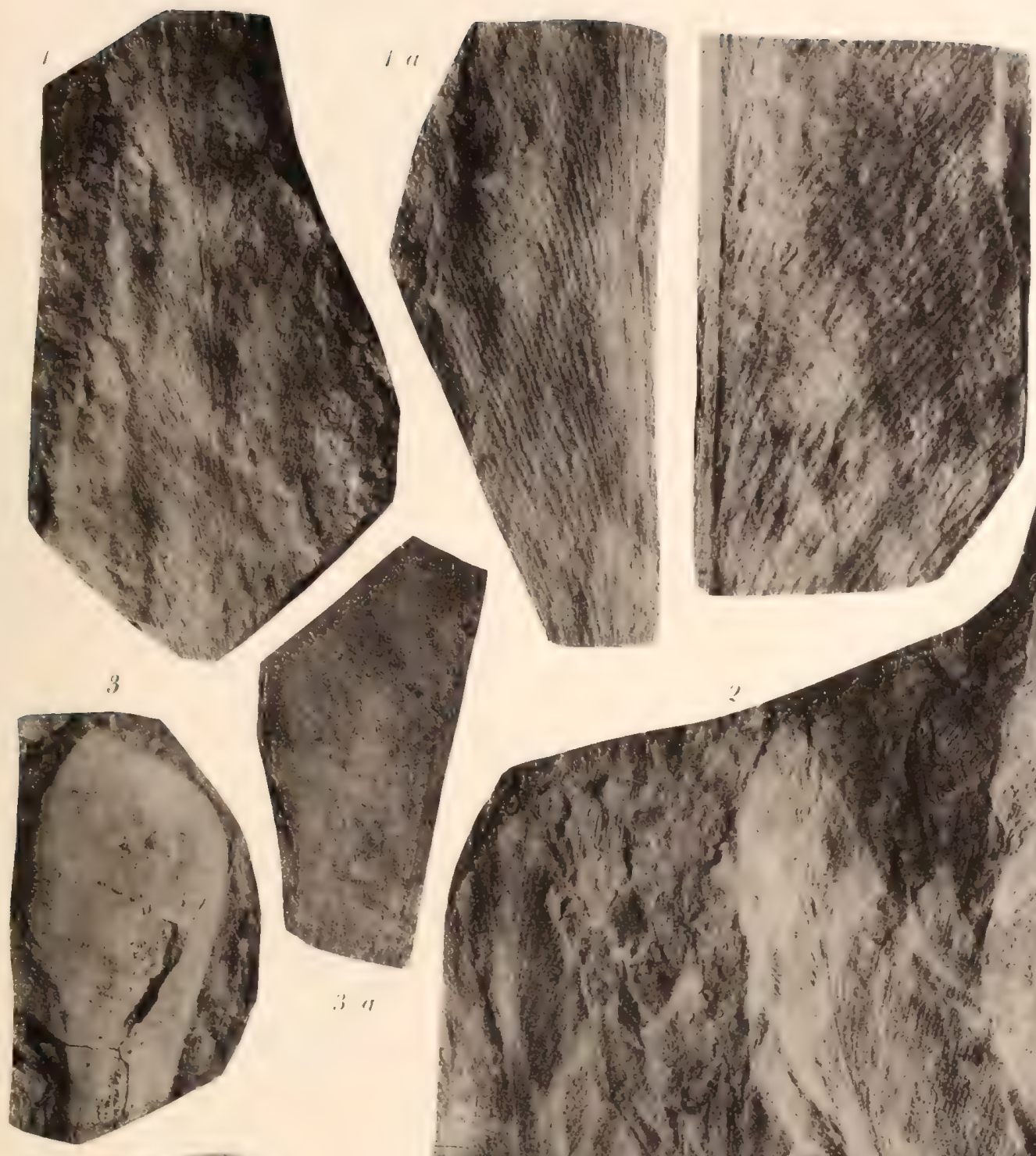

[1. X11
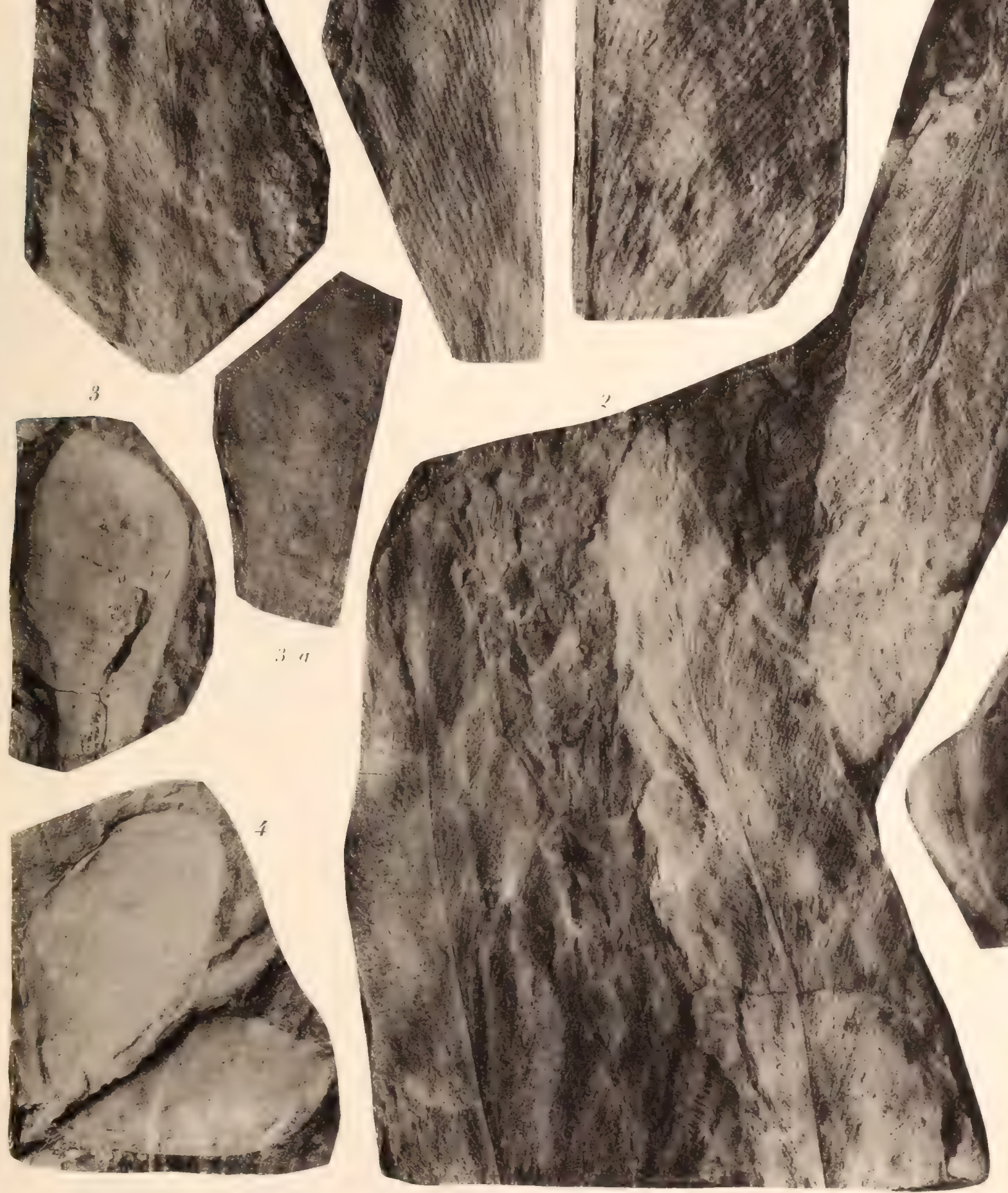

Cliches soluer. 



\section{PLANCHE XVII}




\section{PLANCHE XVII.}

\section{EXPLICATION DES FIGURES.}

Fic. 1. - Woodwardites microlobus Schenk. - Fragments de frondes (figurés Annales des Mines, $8^{e}$ série, t. II, pl. XII, fig. 3).

Hongaÿ, mine Jauréguiberry.

Fig. 2. - Woodwardites microlobus Schenк. - Empreinte d'un fragment de penne. Hongaÿ, mine Jauréguiberry.

FIG. 2 a. - Portion du même échantillon, grossie deux fois.

Fig. 3. - woodwardites microlobus Schenk. - Fragment de fronde.

Mines de Hongaÿ, découvert de Hatou.

Fı. 3 a. - Portion du même échantillon, grossie deux fois.

Fig. 3 b. - Portion du même échantillon, grossie une fois et demie.

Fig. 4. - Woodwardites microlobus Schenk. - Empreinte d'un fragment de penne fertile.

Hongaÿ, vallée orientale de l'OEuf, couche près d'une petite île.

Fig. 4a. - Le mème échantillon, grossi deux fois et demie.

Fig. 4 a'.- Portion du même échantillon, grossie vingt fois, montrant les anneaux des sporanges (cliché Monpillard).

Fig. 5. - Woodwardites microlobus Schenk. - Fragment de fronde (figuré Annales des Mines, $8^{\mathrm{e}}$ série, t. II, pl. XII, fig. (1).

Hongaÿ, mine Jauréguiberry. 

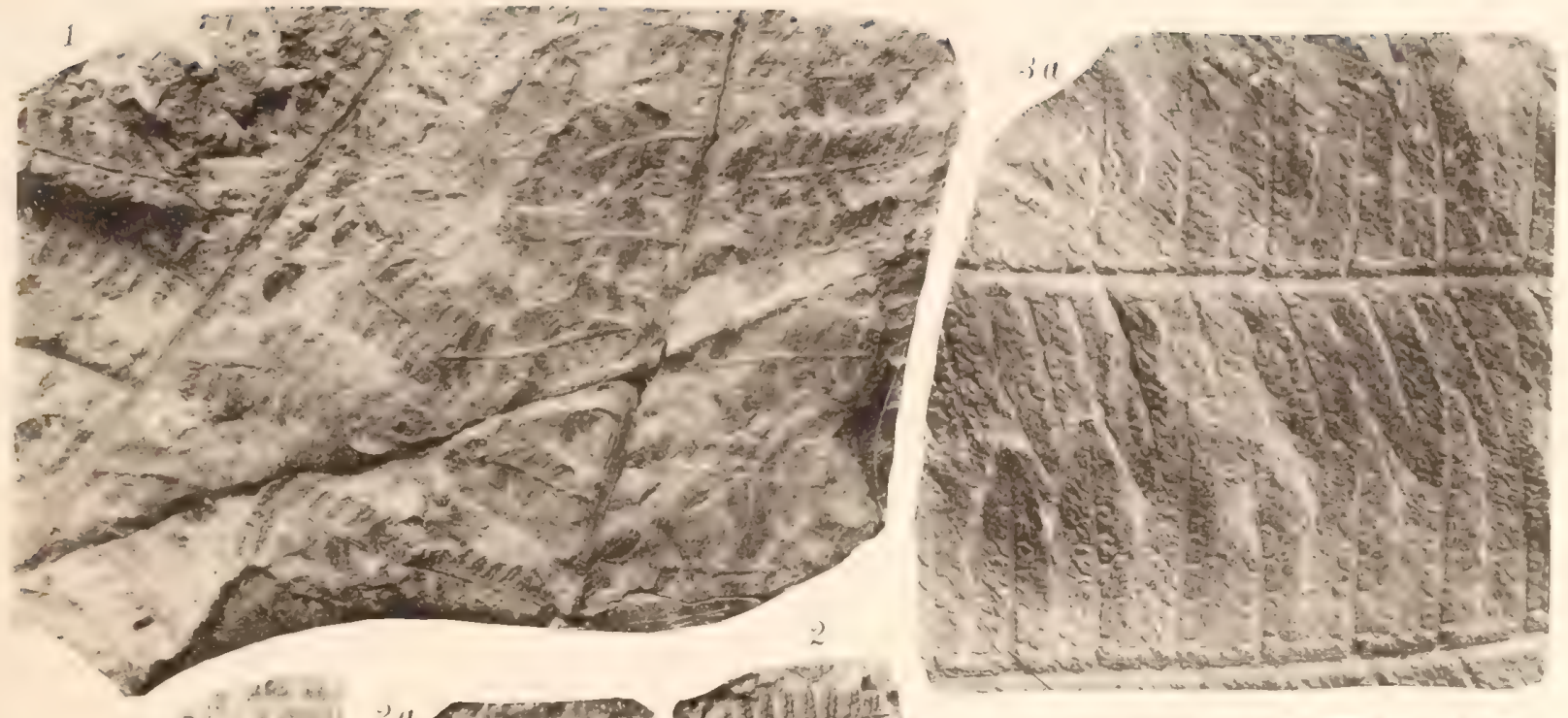
1) 18

तilium

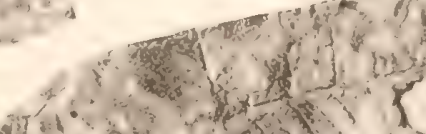

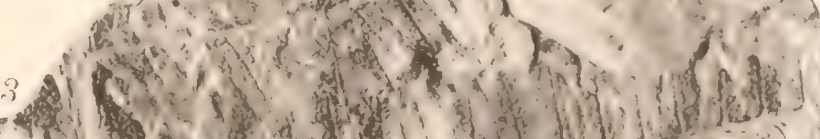
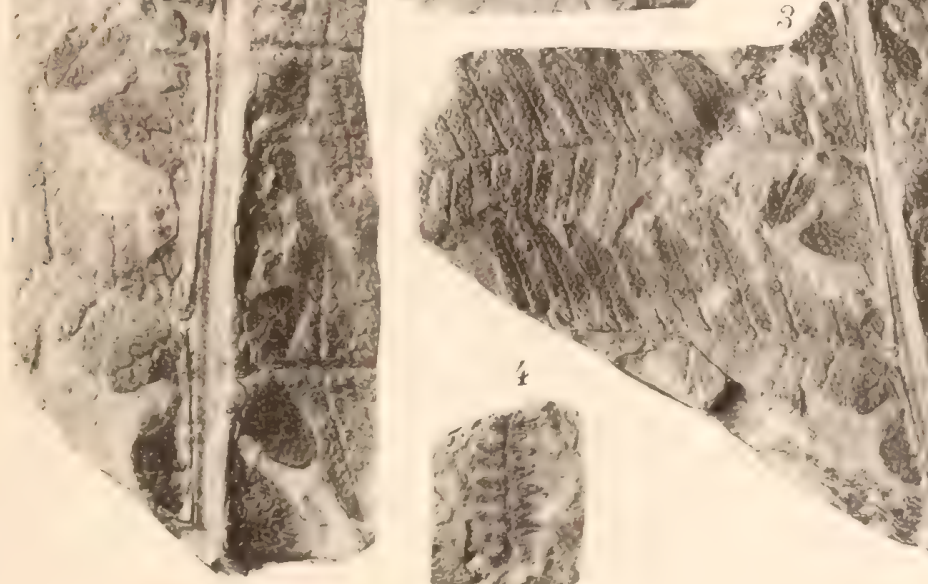

(n)

40

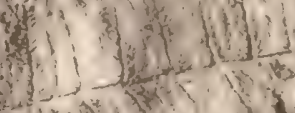

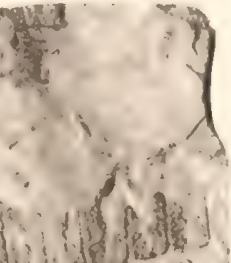
$x+1, i$

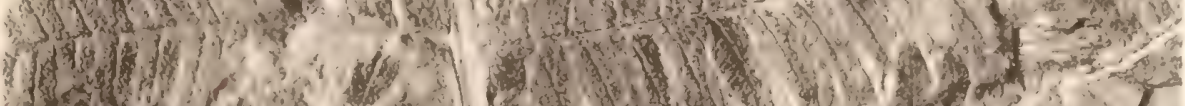

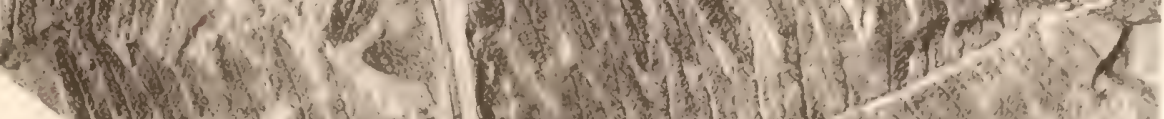

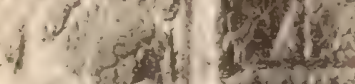

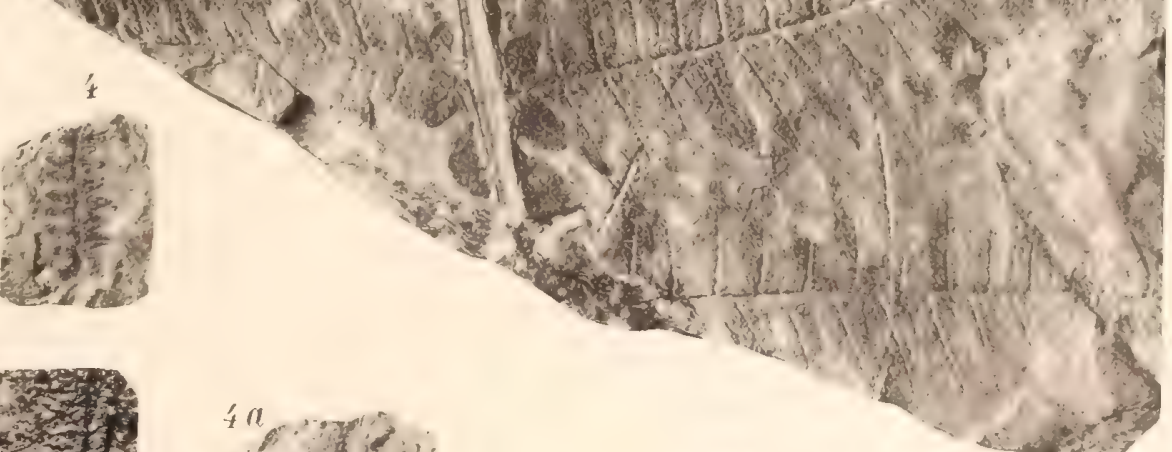

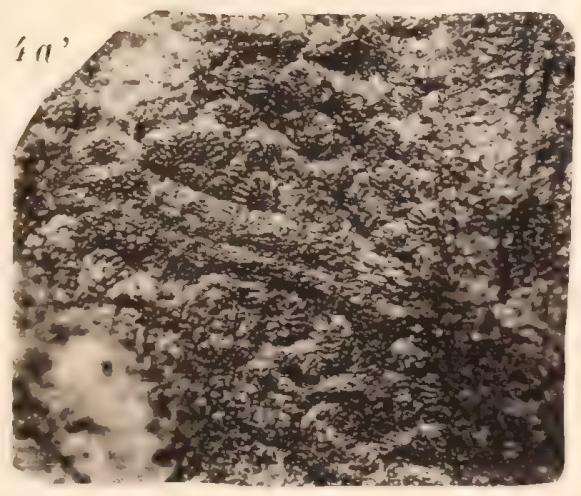

Clichés Sohier
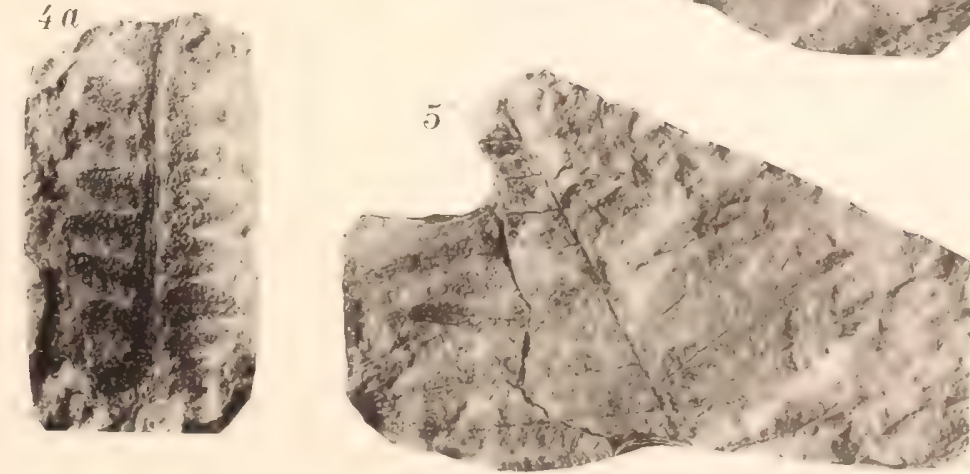

Phototypie Sohier - Lhannpigny \& Marne (Seine) 



\section{PLANGHE XVIII}

GÎTES DE CHARBON DU TONKIN. 


\section{PLANCHE XVIII.}

\section{EXPLICATION DES FIGURES.}

Fig. I. - Dictyophyllum Fuchsi ZeILler. - Portions de frondes. Kébao, mine Rémaury, au mur de la couche U.

Fig. I at - Portion du même échantillon, grossie une fois et demie.

Fig. 2. - Dictyophyllum Fuchsi Zeiller. - Fragments de frondes. Hongaỹ, île du Sommet Buisson, galerie Jean.

F'ig. 2. i. - Portion du même échantillon, grossie une fois et demie.

FII, 2 it. - Portion du mème échantillon, grossie trois fois. 


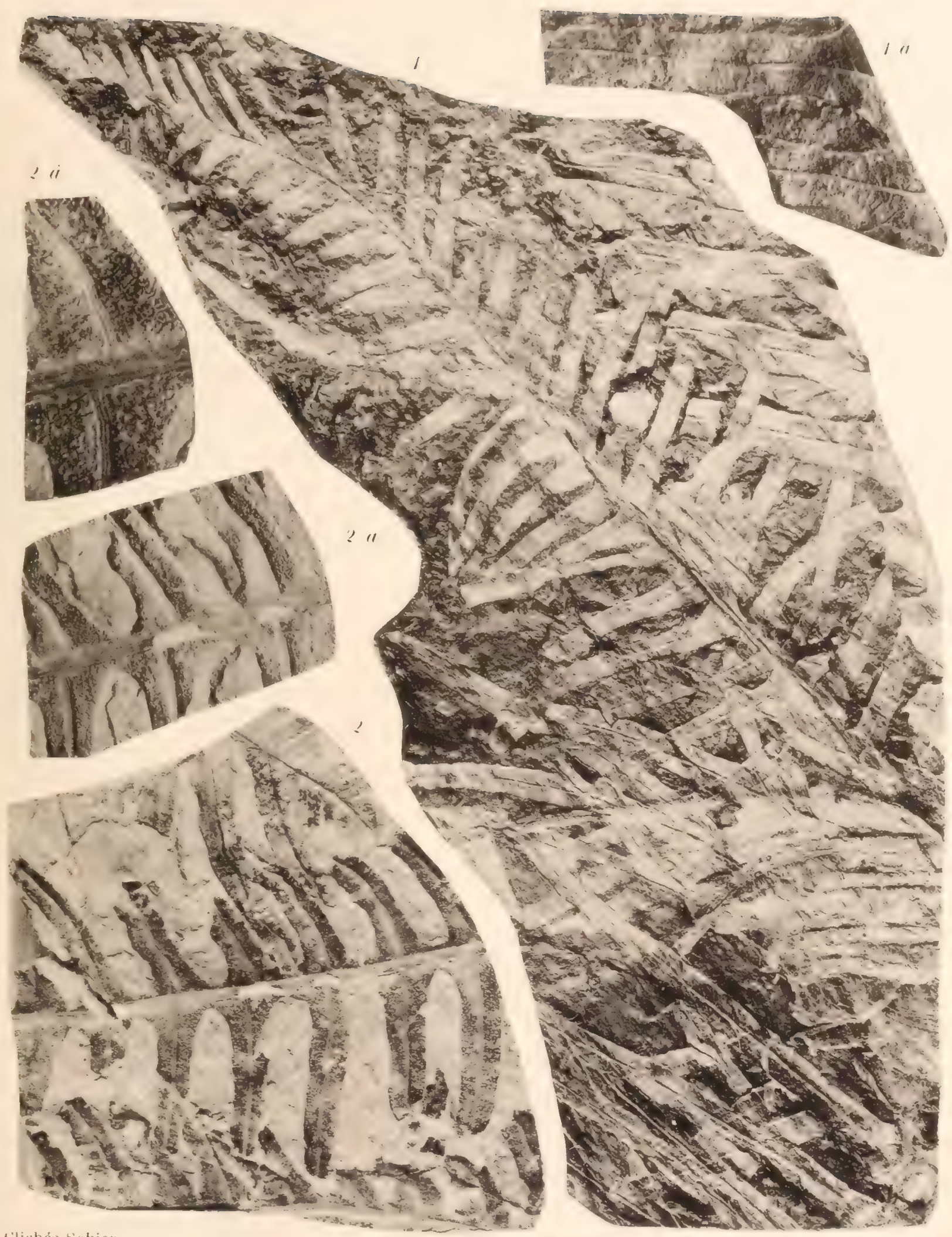



PLANCHE XIX 


\section{PLANCHE XIX.}

\section{EXPLICATION DES FIGURES.}

Fig. 1. - Dictyophyllum Remauryi n. sp. - Partie inférieure d'une penne primaire. Kébao, mine Rémaury.

Fig. 2. - Dictyophyllum Remauryi n. sp. - Portion de penne primaire. Kébao, puits Lanessan. 


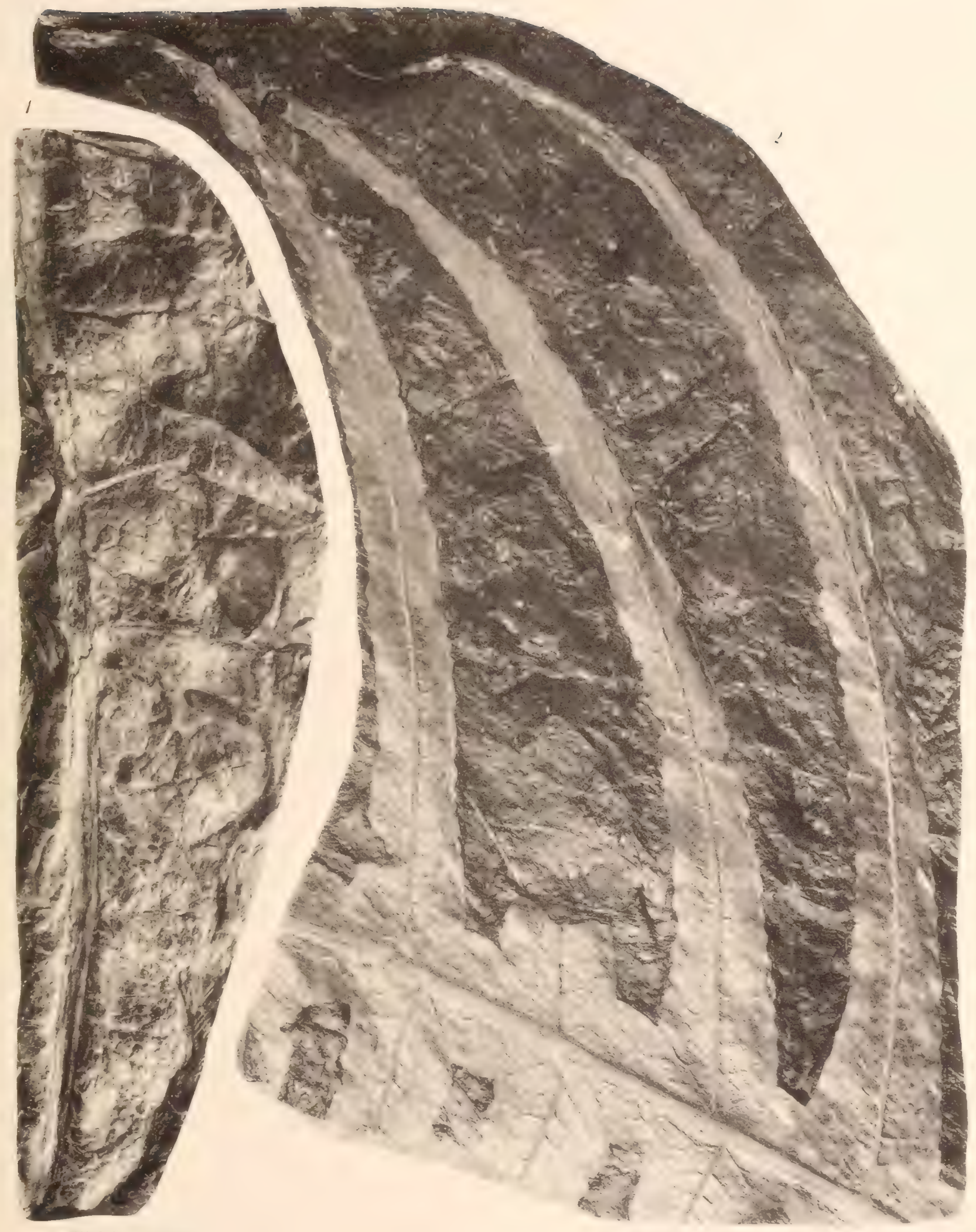

Clichés soldien

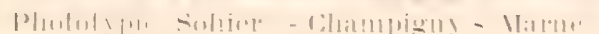



PLANCHE XX 


\section{PLANGHE XX.}

\section{EXPLICATION DES FIGURES.}

Fig. 1 et 2. - Dictyophyllum Remauryi n. sp. - Fragments de frondes. Mines de Hongaÿ, découvert de Hatou.

FIG. 3. - Dictyophyllum Remauryi n. sp. - Portion supérieure d'une penne primaire. Kébao.

Fig. 3 a. - Portion du mème échantillon, grossie trois fois.

Fig. 4. - Dictyophyllum Remauryi n. sp. - Portion de penne primaire. Kébao. 

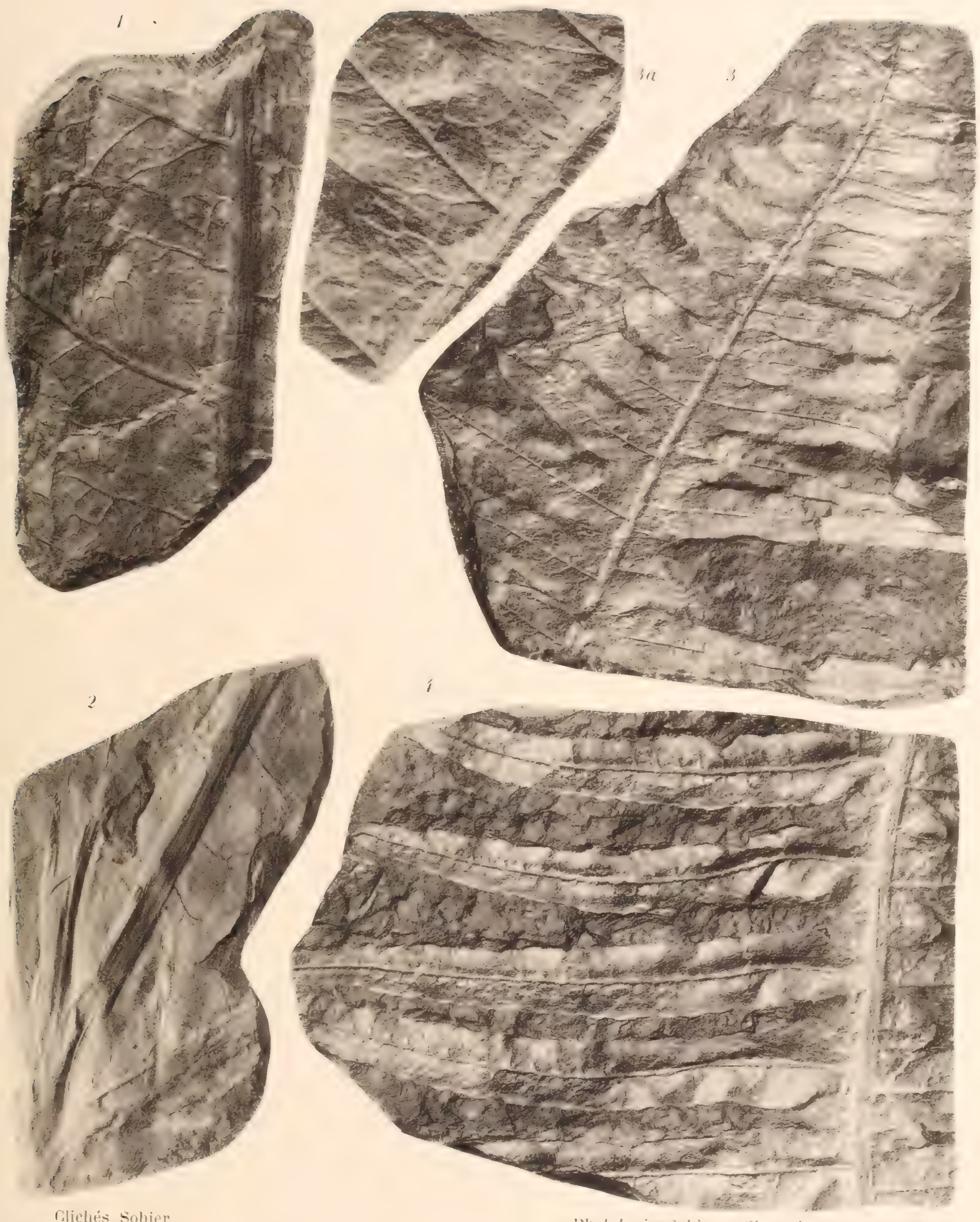

Flichés sohier

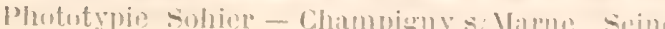


PLANCHE XXI 


\section{PLANGHE XXI.}

\section{EXPLICATION DES FIGURES.}

FIG. 1 of 2. - Dictyophyllum Remauryi n. sp.- Portions inférieure et supérieure d'un même fragment de penne primaire: la portion de droite, fig. 2 , vient se placer à la partie supérieure de la portion de gauche, fig. 1, avec laquelle elle a une partie commune de 1 centimètre environ de longueur. Kébao,

Fig. 1 a, 2a. - Portions du même échantillon, grossies une fois et demie.

FIG. 1 b. - Portion fertile du même échantillon (penne supérieure de gauche de la fig. 1), grossie deux fois et demie. 


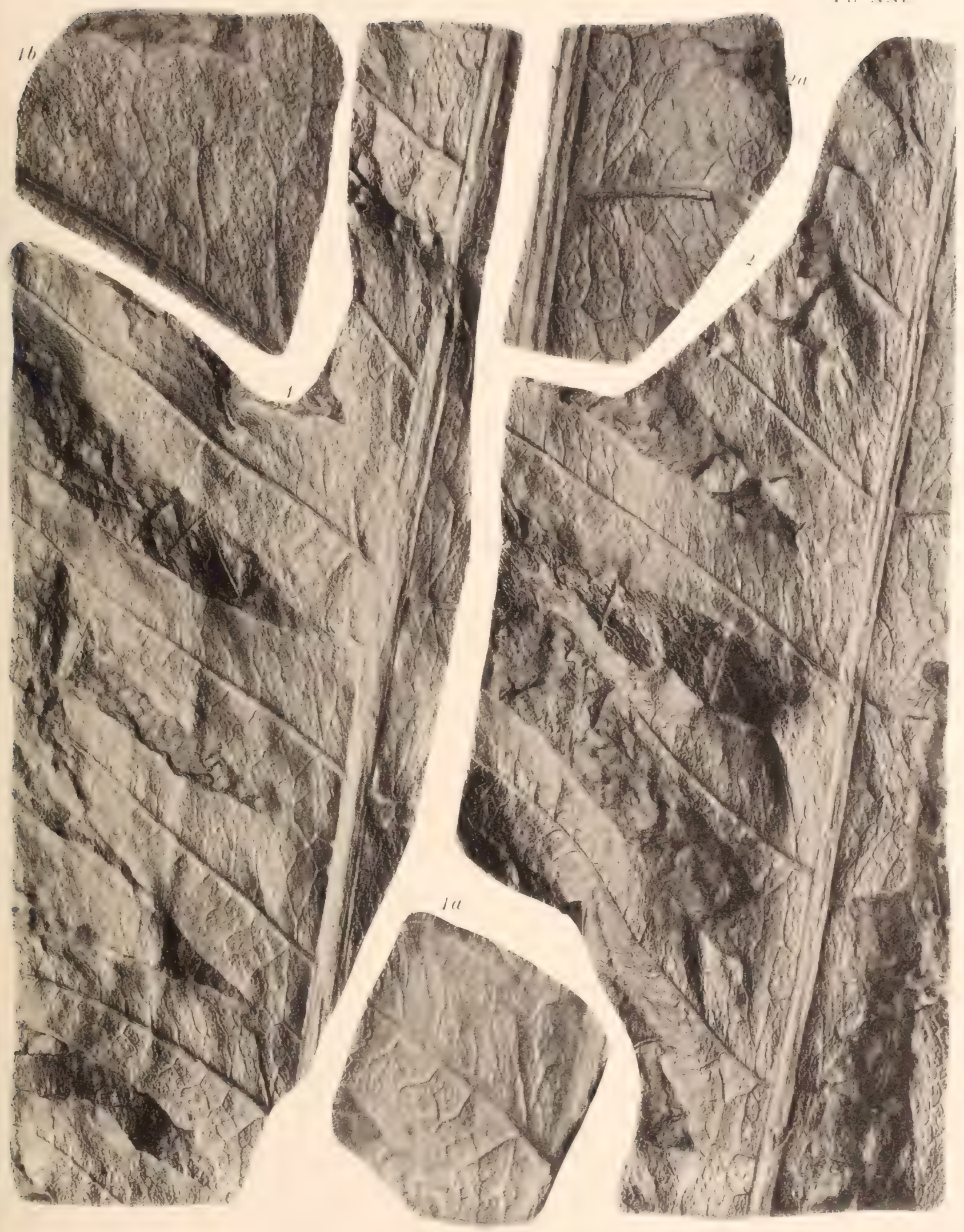





\section{PLANCHE XXII}




\section{PLANCHE XXII.}

\section{EXPLICATION DES FIGURES.}

Fig. 1. - Dictyophyllum Sarrani n, sp. - Fragment de fronde.

Hongaì, île du Sommet Buisson, galerie Jean.

FIG. 1 a et 1 b. - Portions du même échantillon, grossies une fois et demie. 

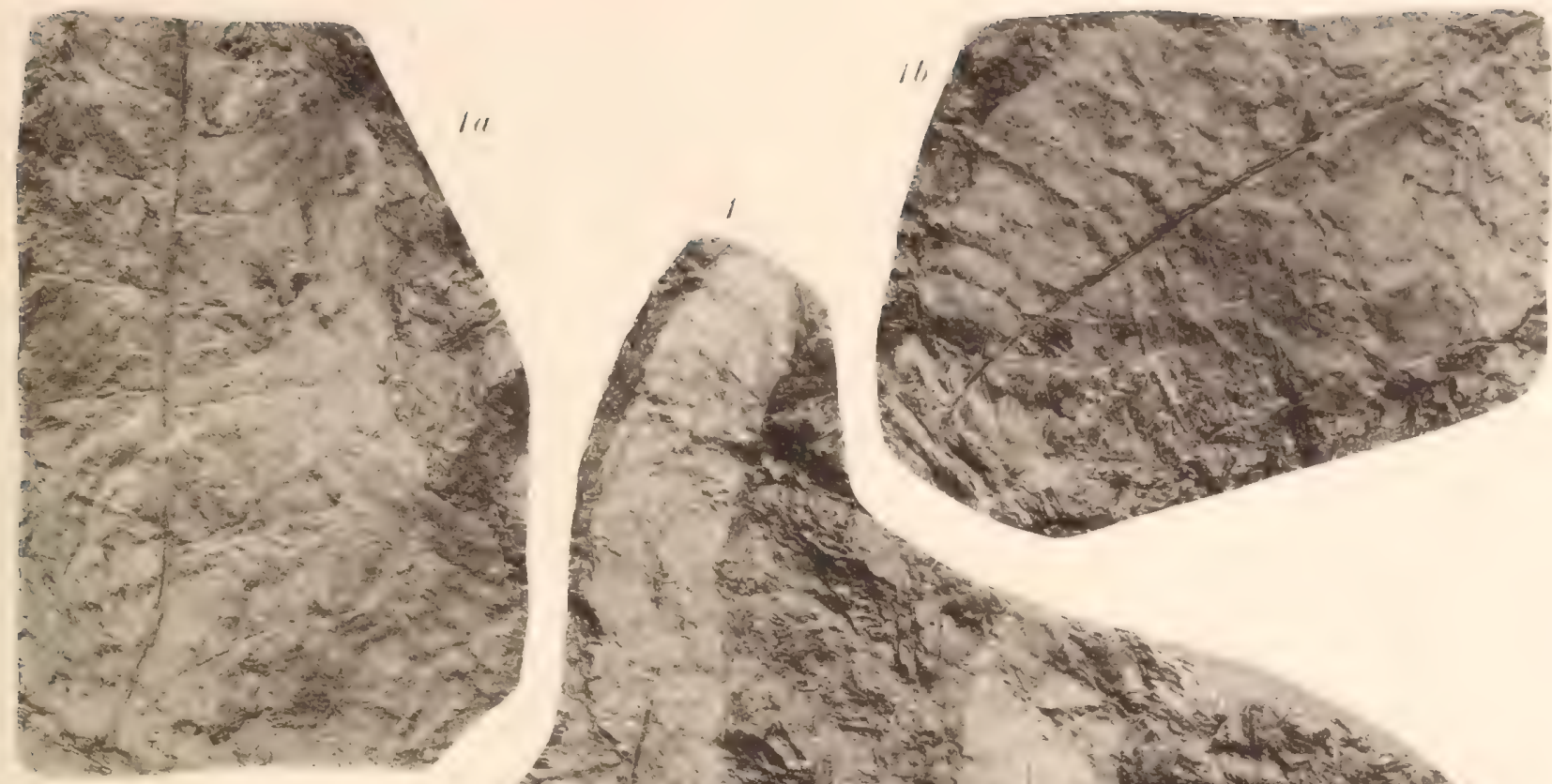

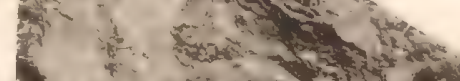

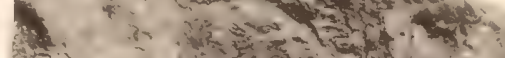

f 2 and

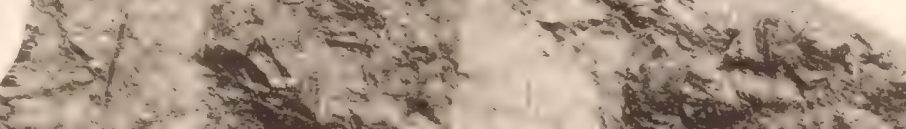

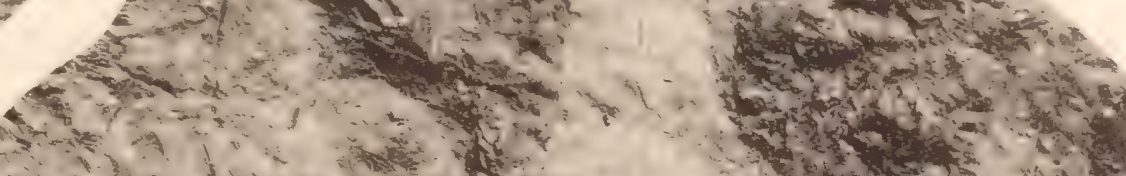

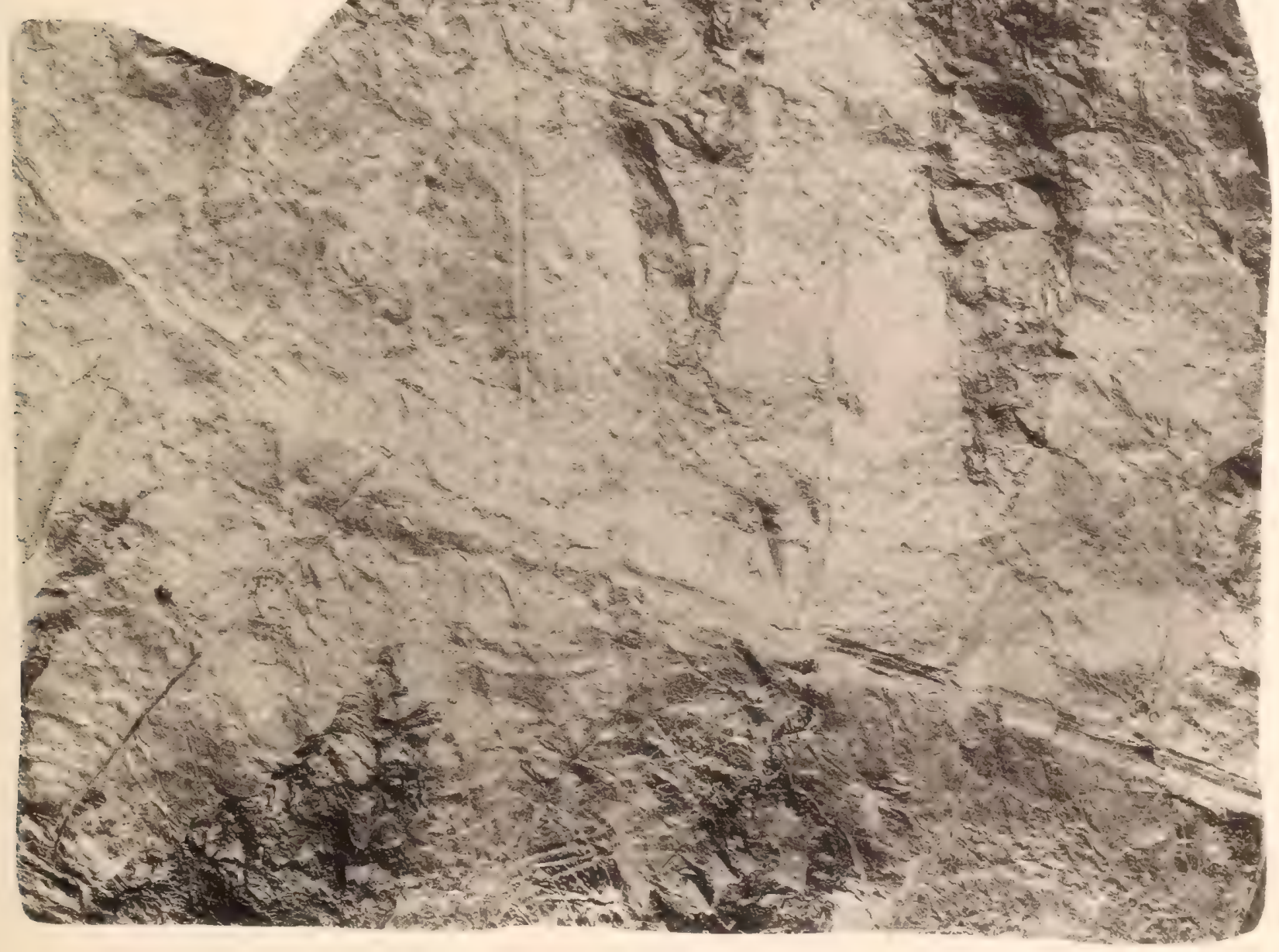

Cliches sohion. 

PLANCHE XXIII 


\section{PLANGHE XXIII.}

\section{FXPIICATION DES FIGURES.}

Fig. 1. - Dictyophyllum Nathorsti n. sp. - Fronde presque complète; le pétiole, re plié en dessous, s'enfonce dans la roche vers la droite.

Mines de Hongaỹ : Hatou, grande couche. 
l. . XXIII

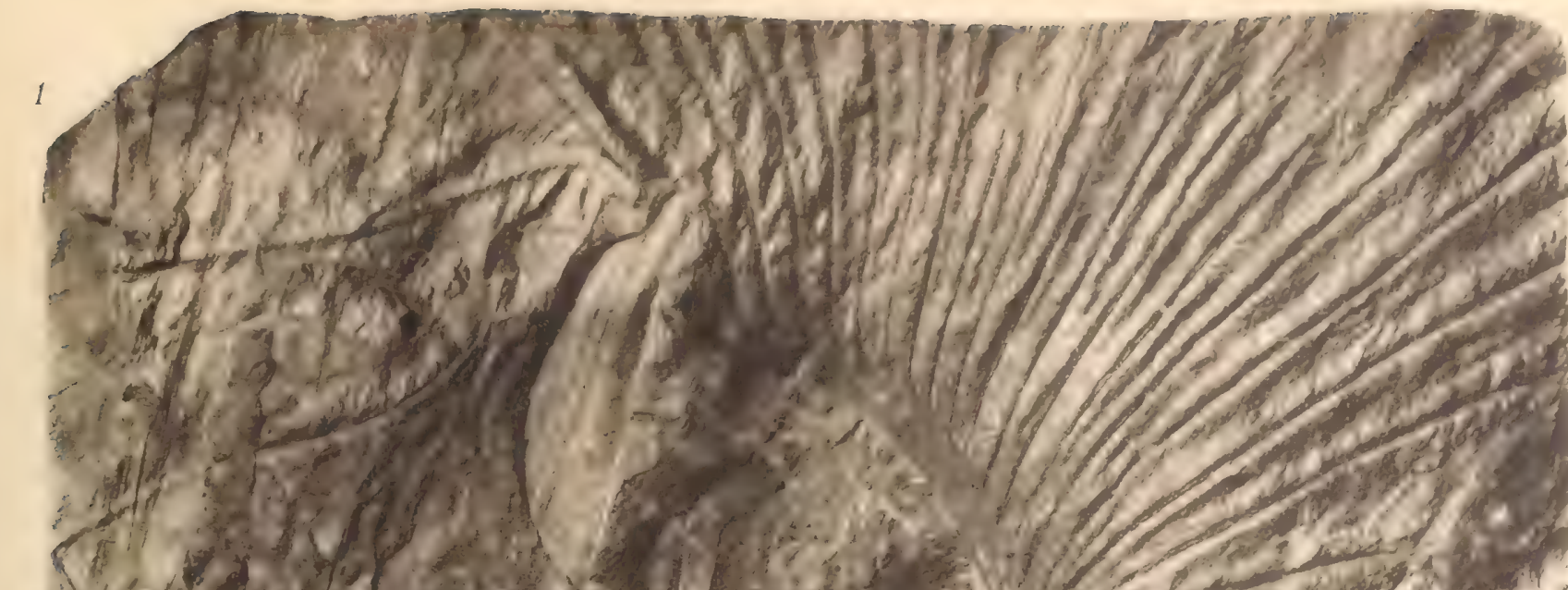

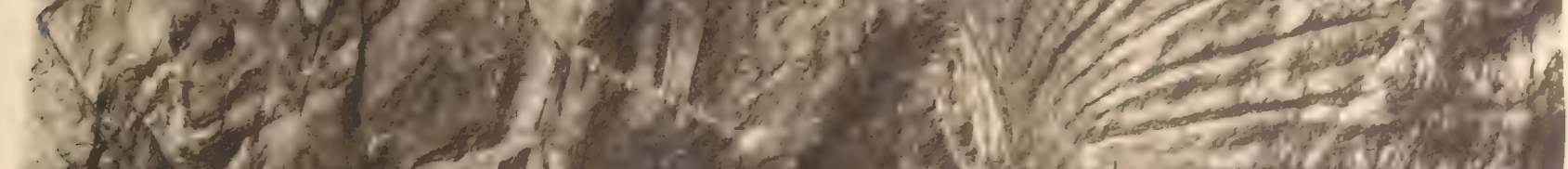

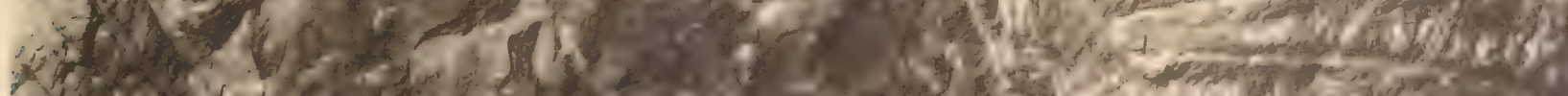

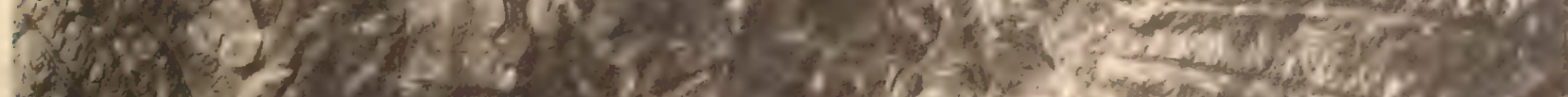

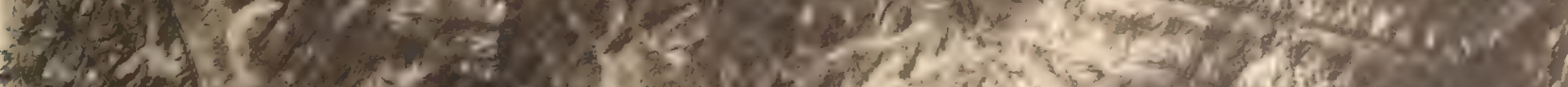

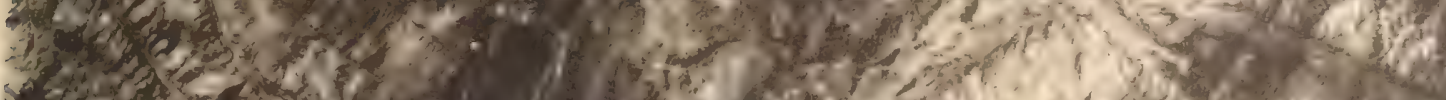

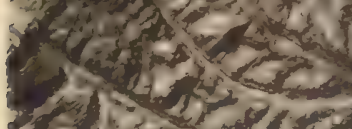

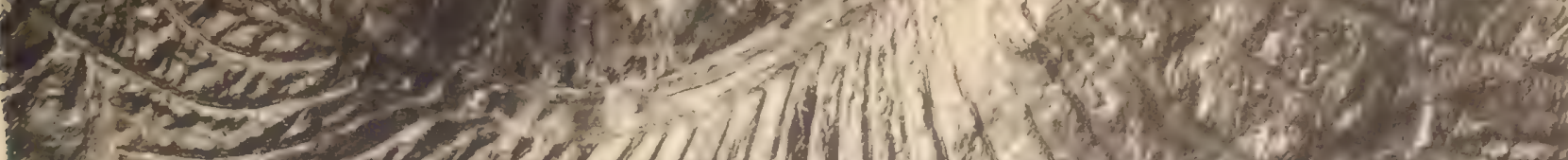

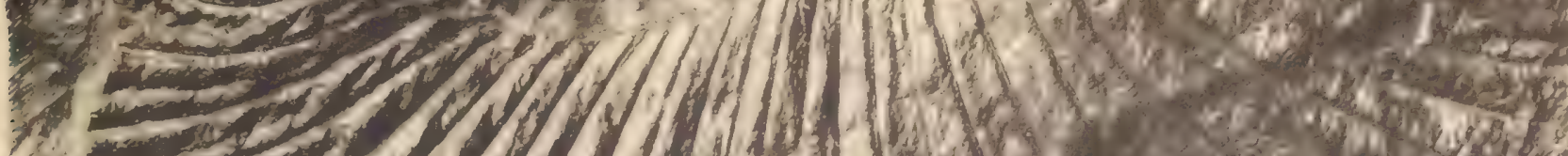

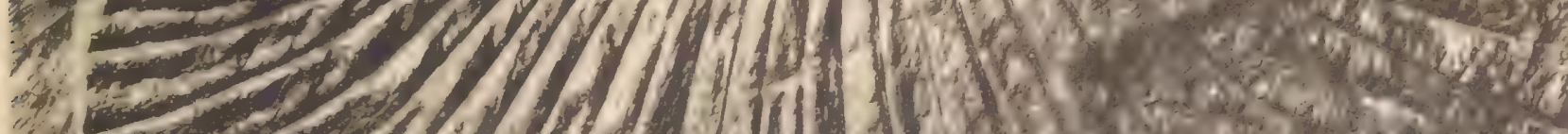

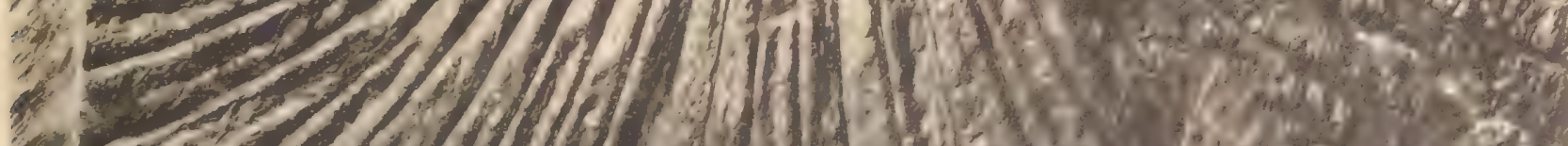
10 (1) If

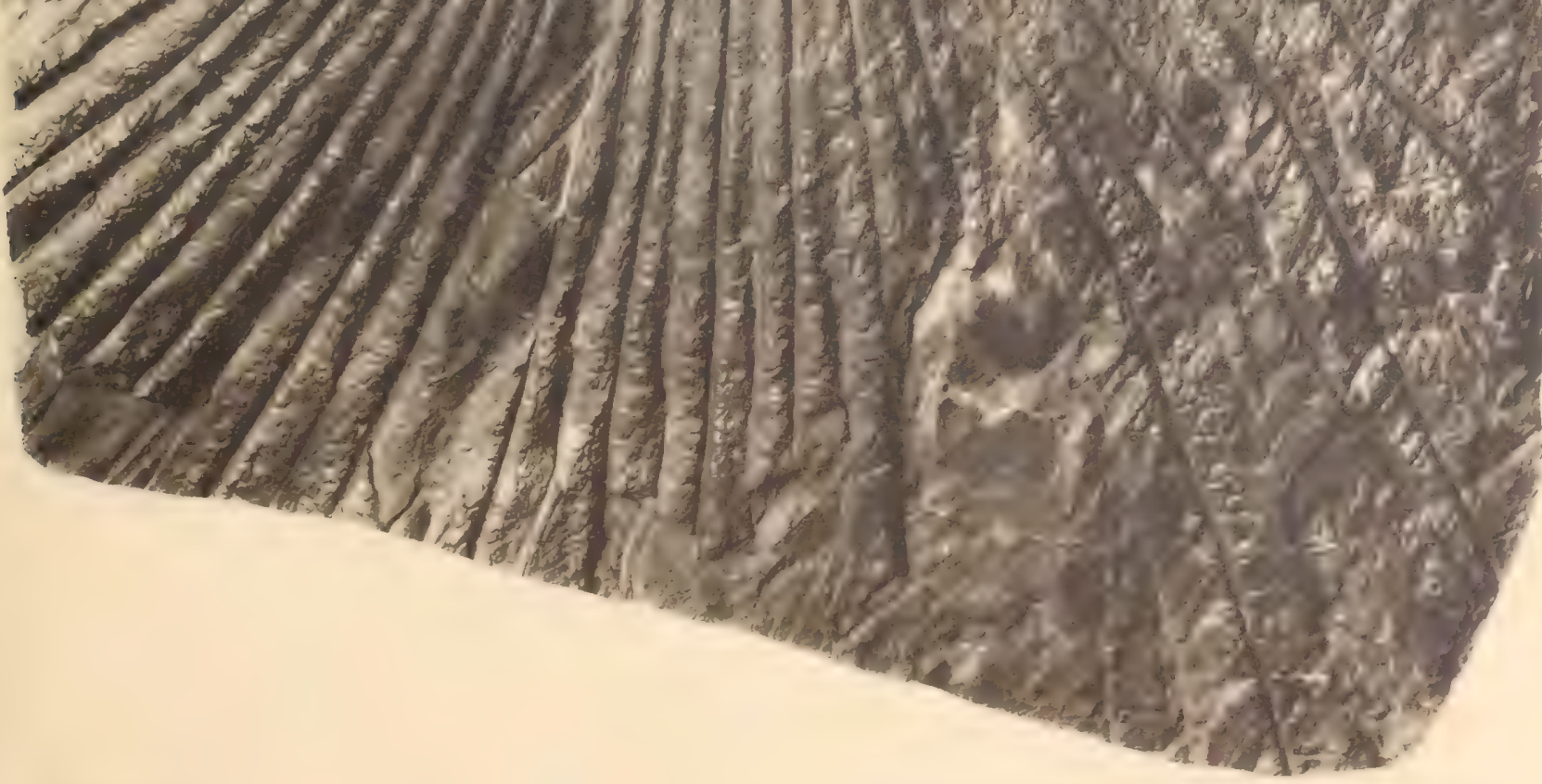



PLANCHE XXIV 


\section{PLANCHE XXIV.}

\section{EXPLICATION DES FIGURES.}

FIG. 1. - Dictyophyllum Nathorsti n. sp. - Réduction, aux trois huitièmes de la grandeur naturelle, d'une grande plaque montrant vers le haut les fragments de deux frondes, et vers le bas une fronde à peu près complète dont une petite partie du limbe reste masquée par le morceau de roche sur lequel se trouve le pétiole.

Mines de Hongaý: Hatou, grande couche, grand banc de schistes. 


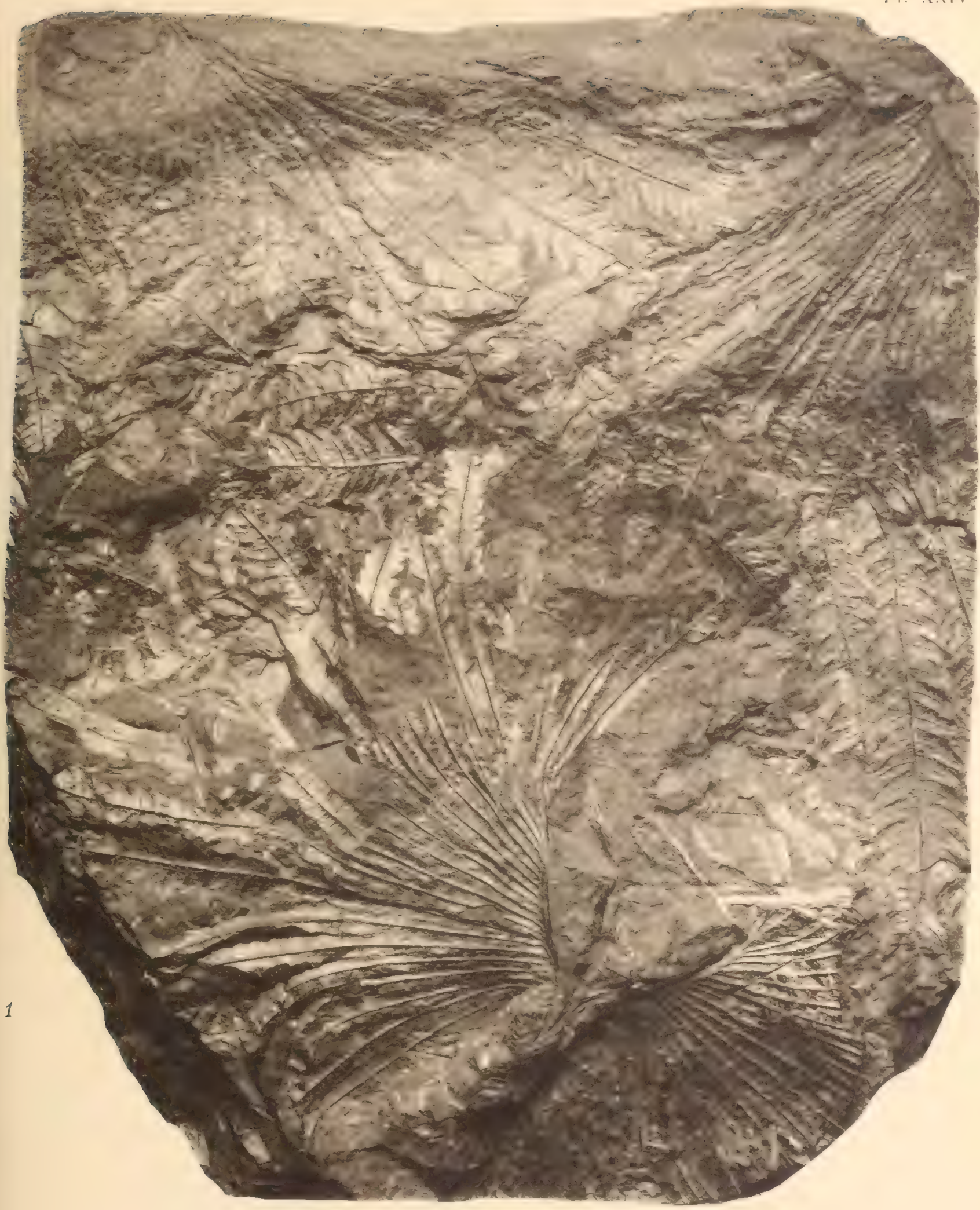



PLANGHE XXV 


\section{PLANCHE XXV.}

\section{EXPLICATION DES FIGURES.}

Fig. 1. - Dictyophyllum Nathorsti n. sp.- Partie inférieure d'une penne primaire. Mines de Hongaÿ: Hatou, grande couche, grand banc de schistes.

FIG. 2. - Dictyophyllum Nathorsti n. sp. - Fragment de penne primaire. Mines de Trang-Back (ancienne concession Schædelin).

FIG. 3. - Dictyophyllum Nathorsti n. sp. - Fragment de penne primaire. Mines de Hongaÿ: Hatou, grande couche, grand banc de schistes.

Fig. 4. - Dictyophyllum Nathorsti n.sp. - Partie supérieure d'une penne primaire. Bassin de Hongaÿ.

FIG. 5. - Dictyophyllum Nathorsti n. sp. - Fragments de pennes primaires. Kébao.

FIG. 5 a. - Portion du même échantillon, grossie une fois et demie.

Fig. 6. - Dictyophyllum Nathorsti n. sp.-Fragment de penne primaire. Mines de Hongaÿ, découvert de Hatou.

FIg. 6a. - Portion du même échantillon, grossie deux fois. 


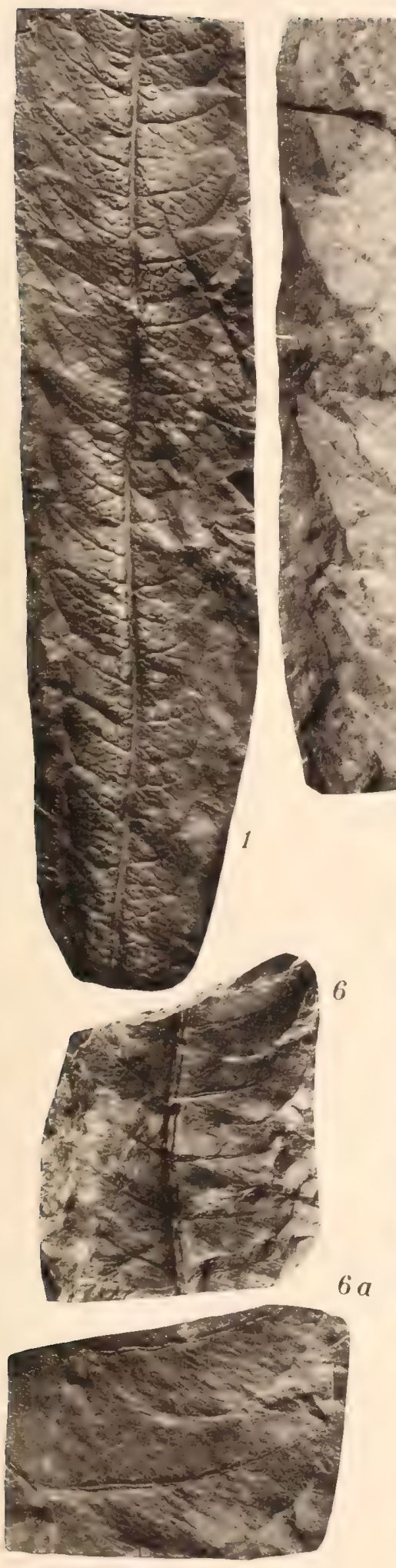

Clichés Sohier
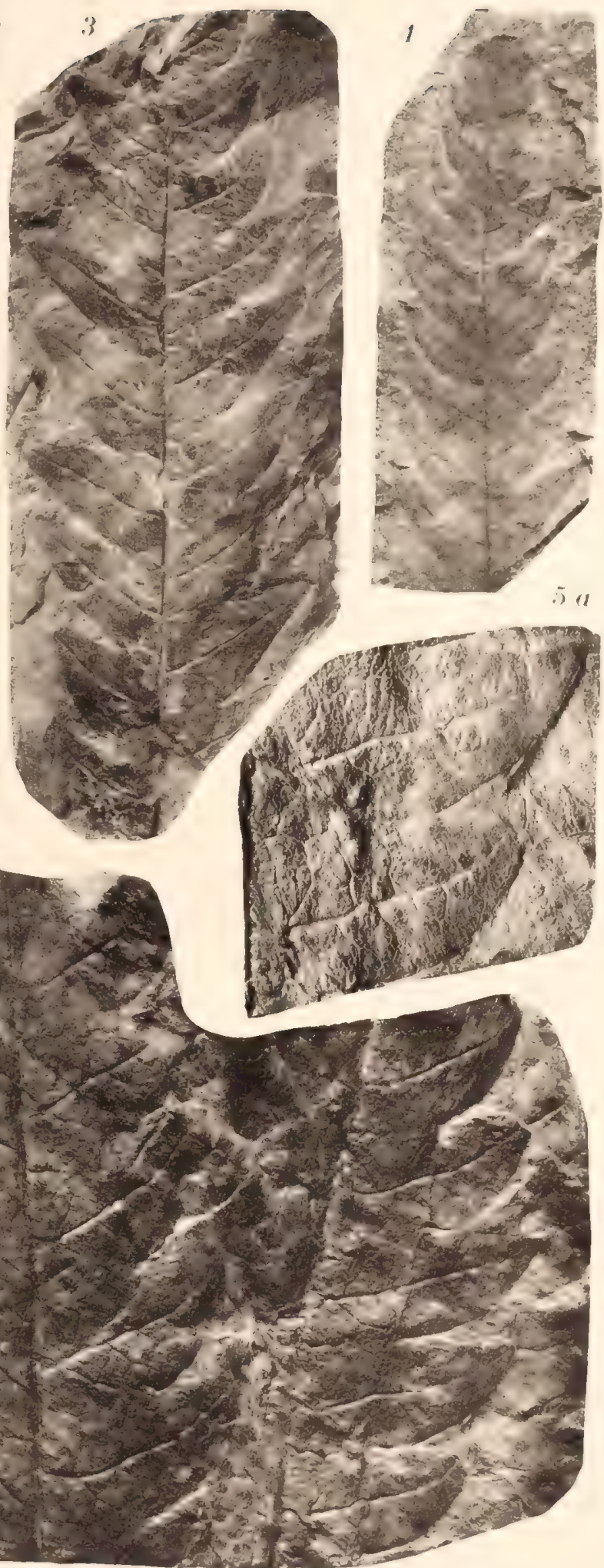

Phototypie Sohier - Champigny-s/Marne (Seine) 



\section{PLANCHE XXVI}




\section{PLANCHE XXVI.}

\section{EXPLICATION DES FIGURES.}

FIG. 1. - Dictyophyllum Nathorsti n. sp. - Frondes provenant d'un jeune pied. Mines de Hongaÿ: Hatou, au toit de la grande couche.

Fig. L a. - Portion du même échantillon, grossie deux fois.

FiG. 2. - Dictyophyllum Nathorsti n. sp. - Portion de fronde.

Mines de Hongaỹ : Hatou, grande couche, grand banc de schistes.

Fig. 2 a. - Portion du mème échantillon, grossie deux fois.

Fig. 3. - Dictyophyllum Nathorsti n. sp. - Portion de fronde fertile.

Kébao, puits Lanessan.

Fig. 3 a et 3 b. - Portions du même échantillon, grossies une fois et demie.

Fig. 3 c. - Portion du même échantillon, grossie vingt fois, montrant les anneaux des sporanges. 
Pl. XXVI
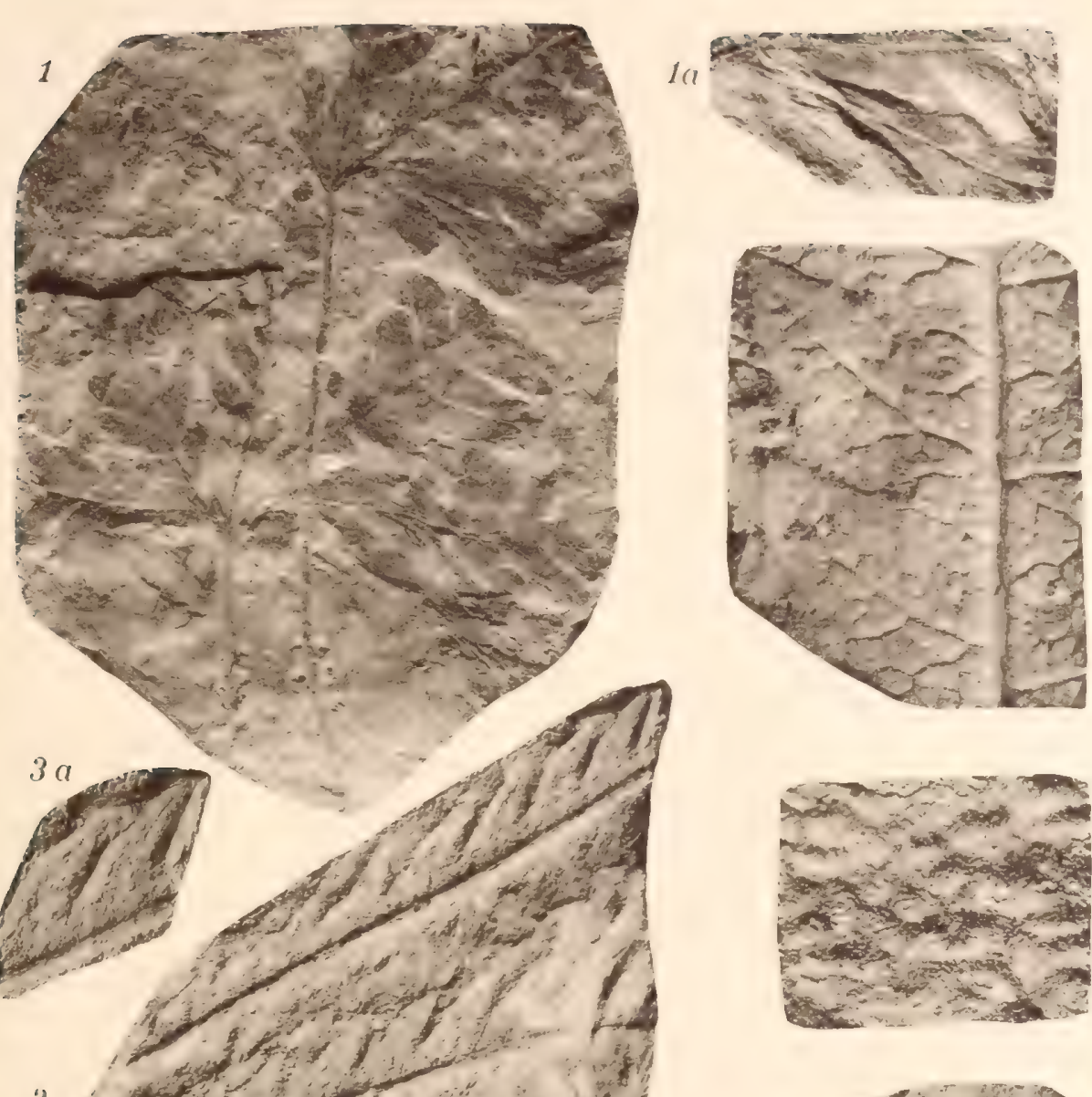

$4+2+2$
$12+2=0$

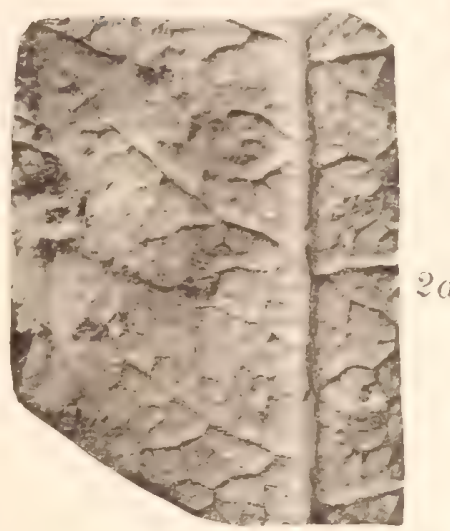

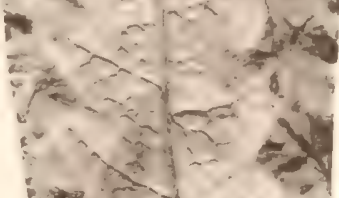

(x) $\simeq-1$

है $\quad 1 \div-2$

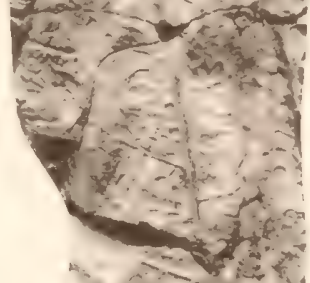

and $x$

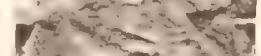

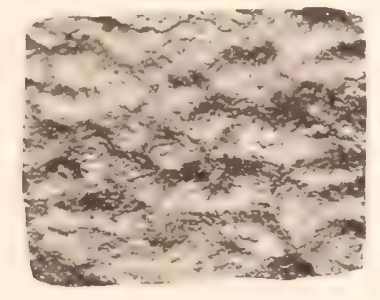

$\therefore-2=$

ic
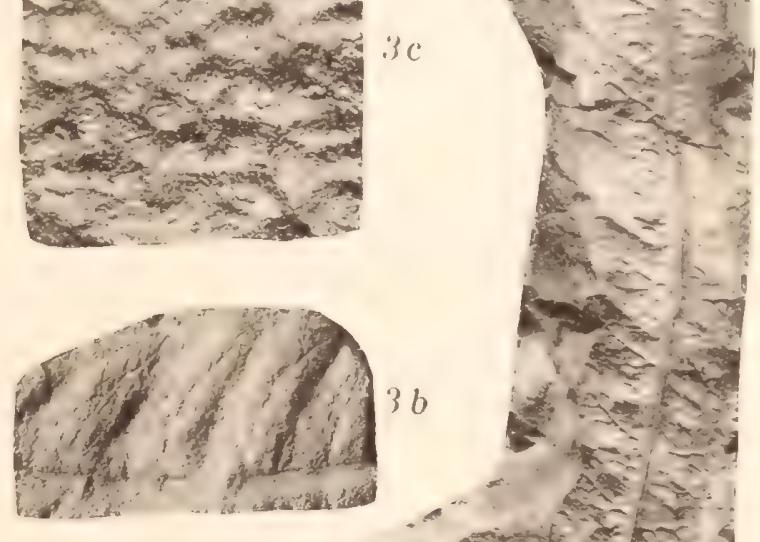

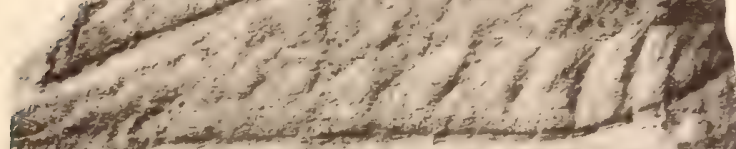
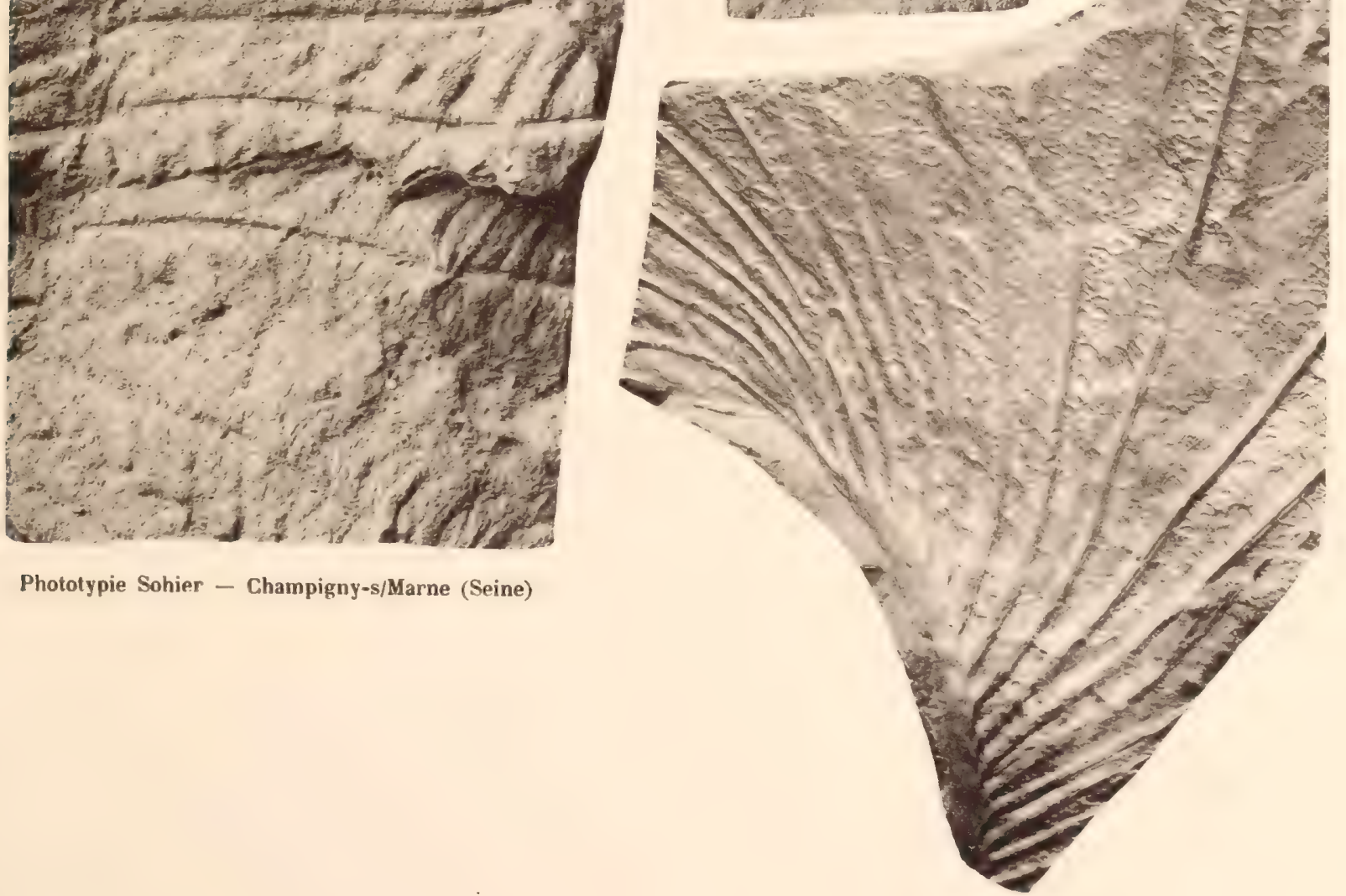



\section{PLANCHE XXVII}




\section{PLANCHE XXVII.}

\section{EXPLICATION DES FIGURES.}

FIG. 1. - Dictyophyllum Nathorsti n. sp. - Portion de fronde dont le pétiole se replie en dessous.

Kébao, puits Lanessan.

Fig. 1 bis. - Pétiole de la même fronde, vu sur la tranche inférieure de l'échantillon.

FIG. 2. - Glathropteris platyphylla Gokppert (sp.). - Fragment de penne primaire. Bassin de Hongaỹ.

FIG. 3. - Glathropteris platyphylla Goeppert (sp.). - Bord latéral inférieur d'une fronde, montrant la naissance des pennes primaires (figuré Revue générale de Botanique, t. IX, pl. 21, fig. 6).

Kébao, puits Lanessan. 

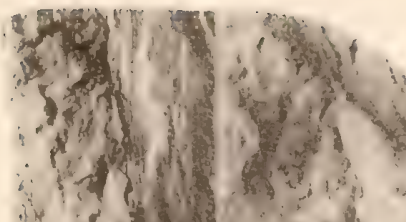

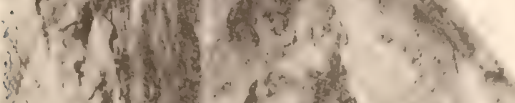

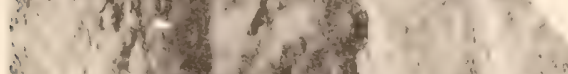

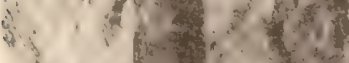

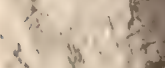

i i

3 in 3

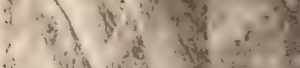

is

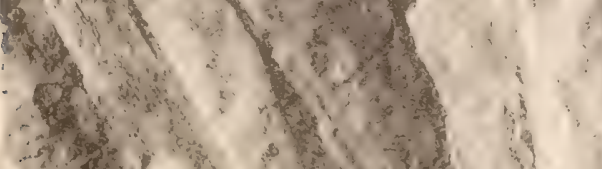

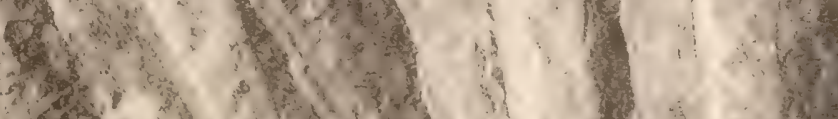

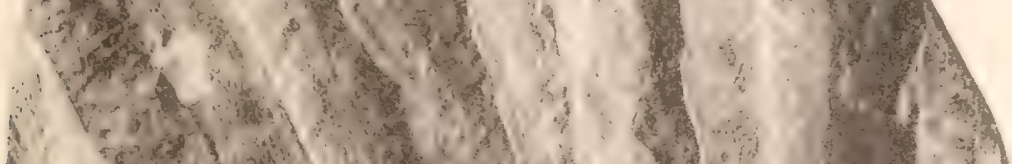
A 3 (2)
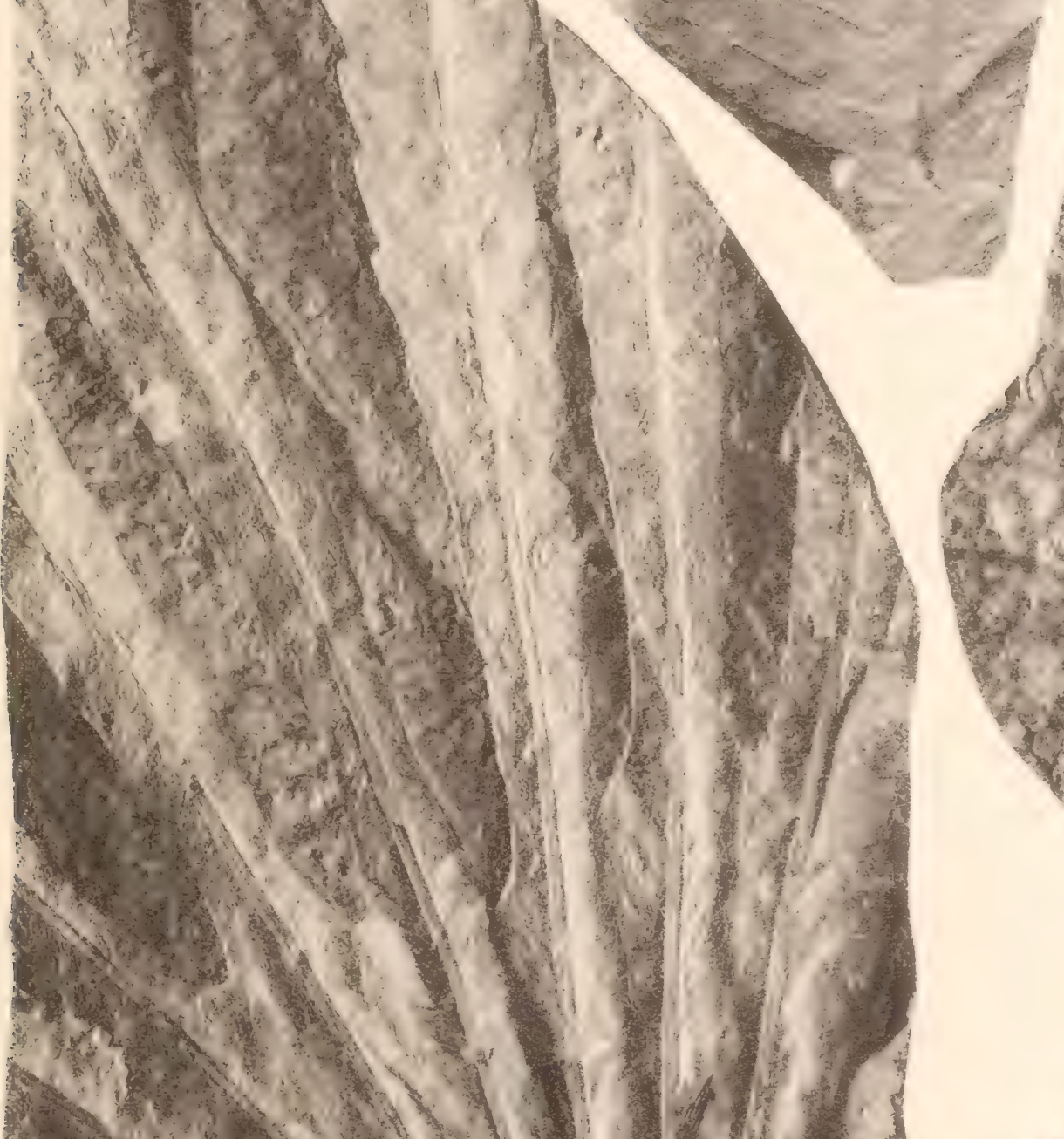

Met

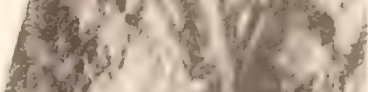
(a)

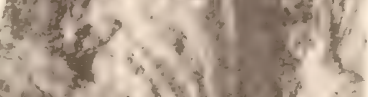

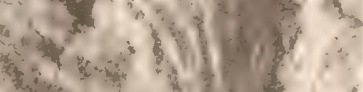
3. $(3)$ in $\rightarrow$ ins ing $\left(x^{2}\right)$
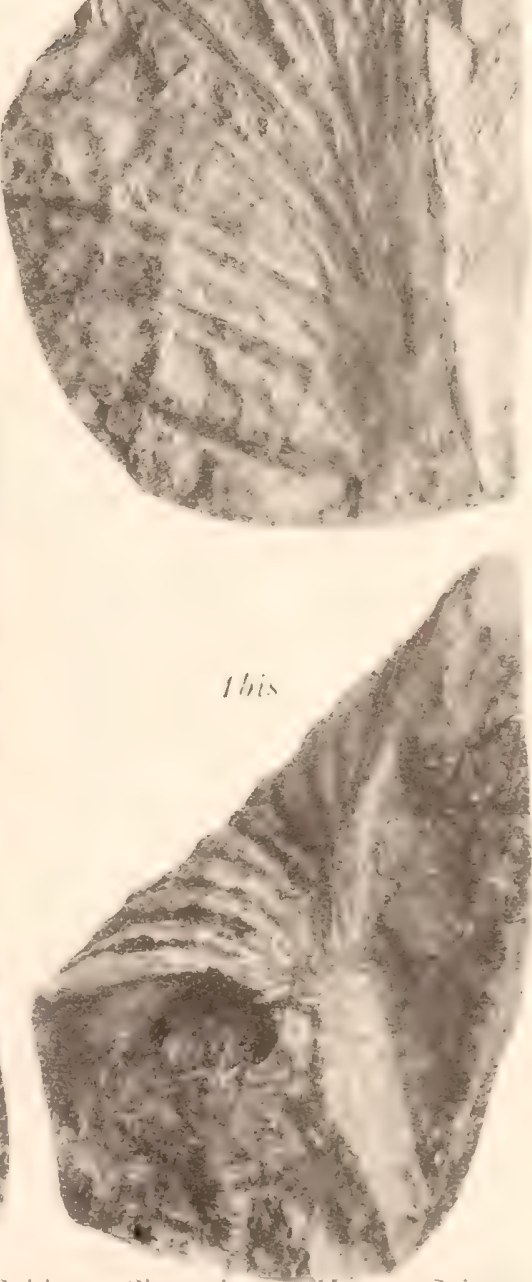

Clichés solier

- Champigny s Vame 



\section{PLANCHE XXVIII}




\section{PLANCHE XXVIII.}

\section{EXPLICATION DES FIGURES.}

FIG. 1. - Clathropteris platyphylla GoKPPERT (sp.). - Fragment d'une penne primaire. Mines de Hongaÿ: Gia-Ham, près Hatou.

Fig. 2. - Glathropteris platyphylla Goeppert (sp.). - Fragments de pennes primaires. Kébao, mine Rémaury, au mur de la couche $\mathrm{S}$.

FIG. $3 \mathrm{a}, 3 \mathrm{~b}, 3 \mathrm{c}$. - Dictyophyllum Nathorsti n. sp. - Fronde repliée sur elle-même, vue sur ses deux faces tatérales (fig. 3a et 3c), et vue de dos (fig. 3b). Kébao, mine Rémaury, couche V. 


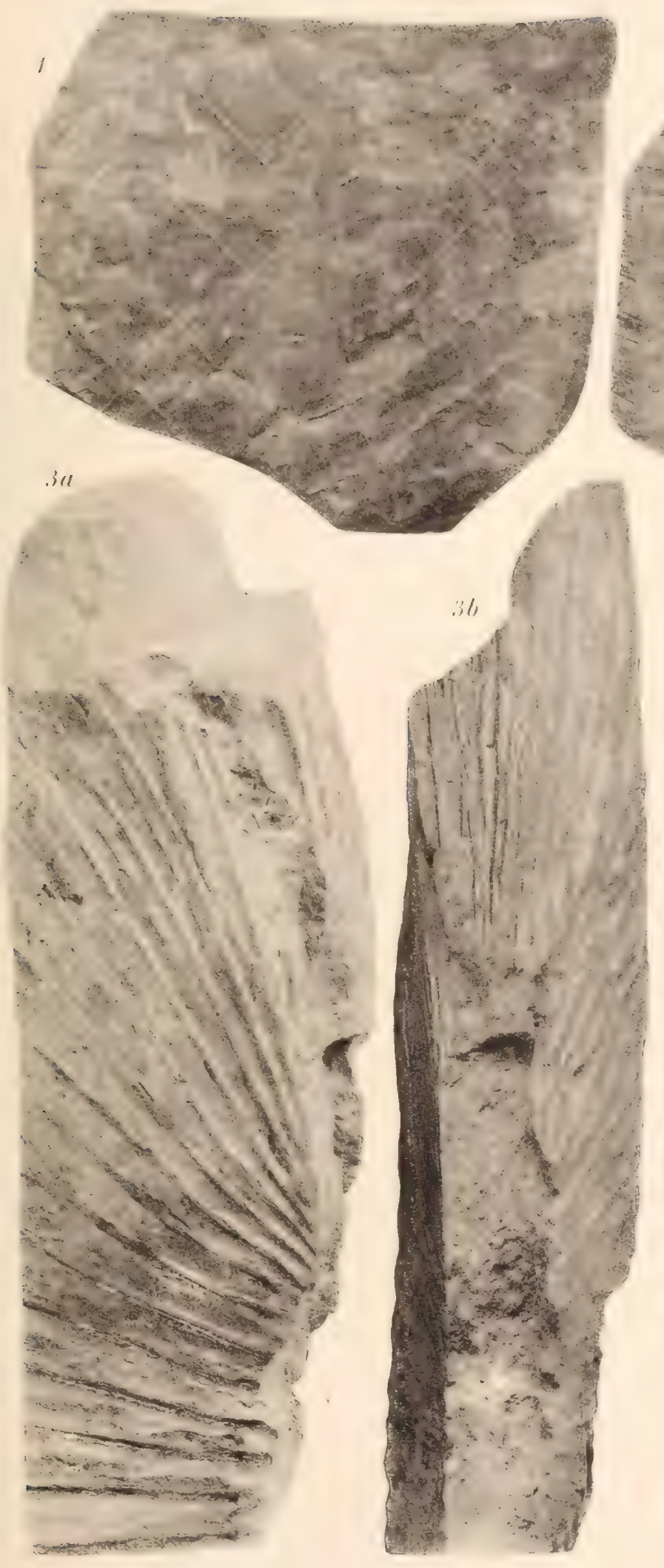

Clichés sohien

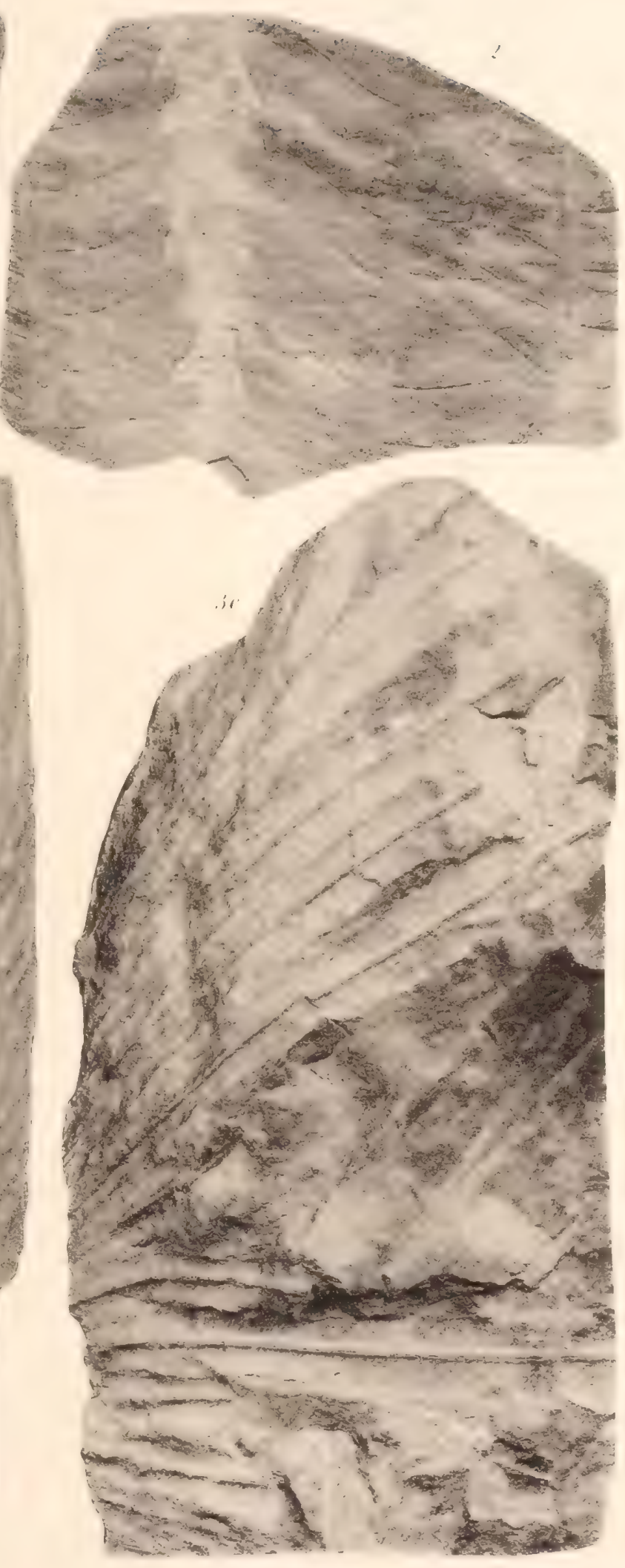

Phototypie sohitp - lihampignys Malme 

PLANCHE XXIX 


\section{PLANCHE XXIX.}

\section{EXPLICATION DES FIGURES.}

Fig. 1. - Clathropteris platyphylla Goépert (sp.). - Fragment de penne primaire. Kébao.

Fig. 2. - Glathropteris platyphylla Goeppert (sp.). - Portion de fronde.

Kébao, système inférieur, au toit de la couche $n^{0} 1$, sous le gros banc de congtomérat.

Fig. 3 et 4. - Clathropteris platyphylla Göppert (sp.). - Fragments de pennes primaires présentant des anomalies de nervation.

Mines de Hongaÿ, découvert de Hatou. 


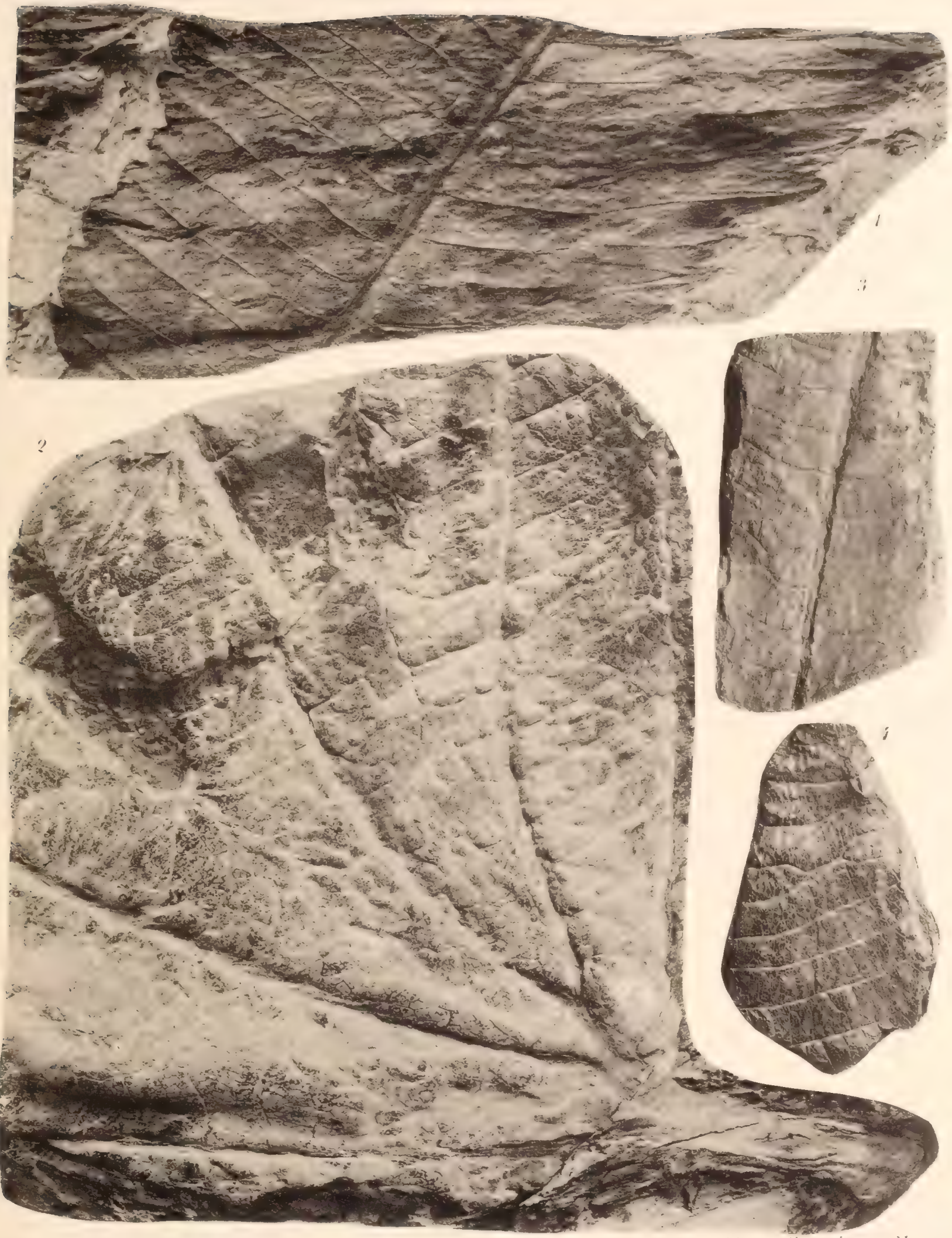

Clichés Sohier 

- 


\section{PLANCHE XXX}




\section{PLANGHE XXX.}

\section{EXPLICATION DES FIGURES.}

Fig. 1. - Glathropteris platyphylla Goeppert (sp.). - Partie supérieure d'une penne primaire.

Mines de Hongaÿ : Hatou, grande couche.

Fig. 2. - Clathropteris platyphylla Goeppert (sp.). - Fragment de penne primaire. Mines de Hongaÿ: Hatou, grande couche.

Fifi. 3. - Glathropteris platyphylla Gorppert (sp.). - Fragment de penne primaire. Kébao.

Fig. 4 à 8 . - Clathropteris platyphylla GoEpPERT (sp.). - Fragments de pennes primaires.

Mines de Hongaÿ : Nagotna, an toit de la couche Bavier. 

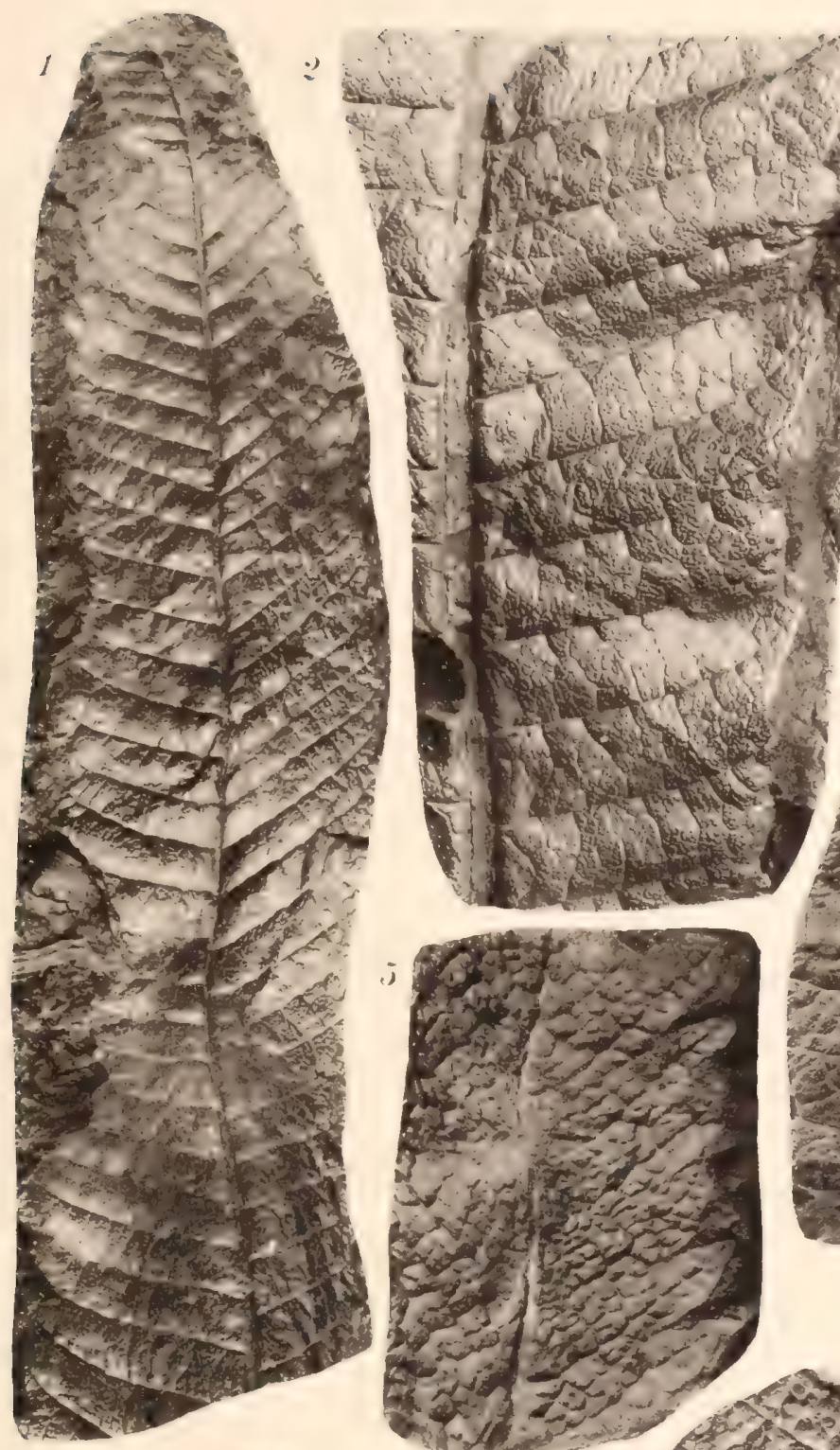

Pl. XXX
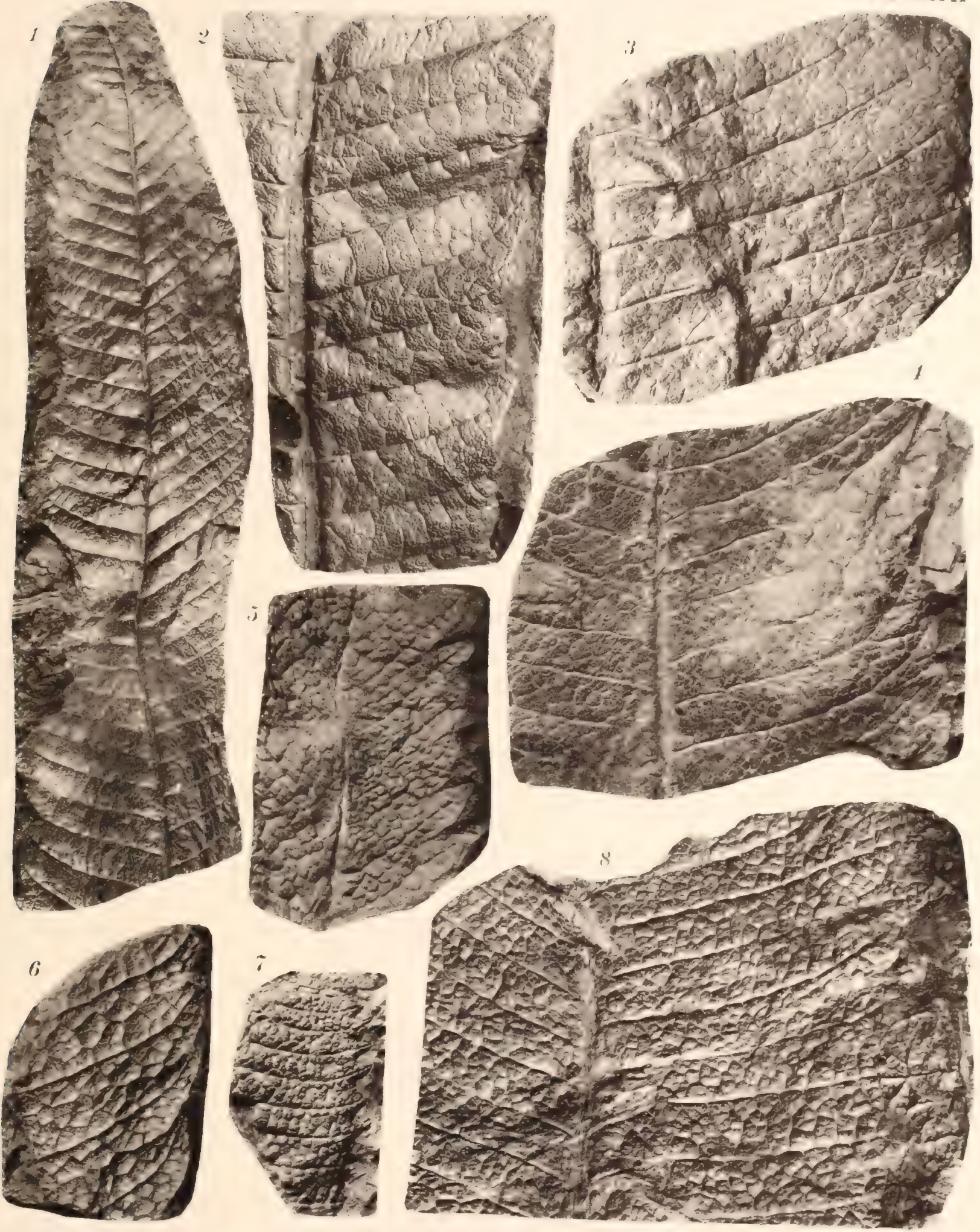

Clichés Sohier.

Phototypie Sohier - Champigny-s/Marne (Seine) 



\section{PLANCHE XXXI}




\section{PLANCHE XXXI.}

\section{FXPLICATION DES FIGURES.}

Fig. 1. - Clathropterts platyphylla Goeppert (sp.). - Fronde presque complète, en partie repliée sur elle-même; on remarque au bas de la figure l'origine des pennes primaires de la région médiane, vues par leur face supérieure, qui étaient engagées dans la roche et qui ont été mises à nu sur 8 à 9 centimètres de longueur; à droite et à gauche, les pennes latérales repliées sur la région médiane et vues par leur face inférieure. Échantillon réduit aux deux cinquièmes de grandeur naturelle; il est représenté en vraie grandeur, du moins partiellement, sur la planche XXXII-XXXIII.

Mines de Hongay : Hatou, grande couche, grand banc de schistes. 


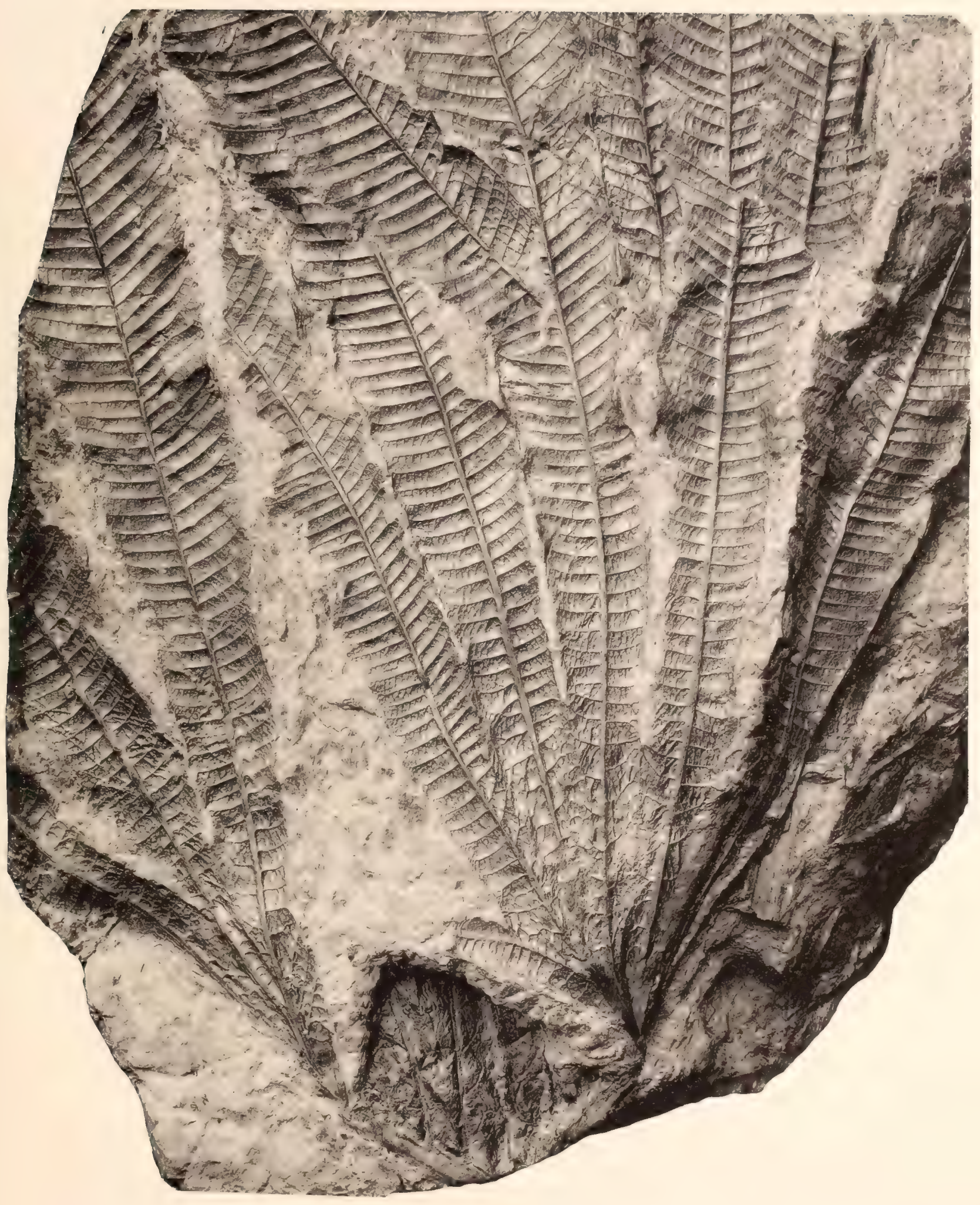





$$
-
$$




\title{
PLANCHE XXXII-XXXIII.
}

\author{
EXPLICATION DES FIGURES.
}

Fig. 1. - Clathropteris platyphylla Gozppert (sp.). - Portion d'une fronde montrant, vers le bas, du côté gauche, une partie de la région médiane mise à nu par l'enlèvement de la roche, et, sur les côtés, les régions latèrales repliées sur la région médiane et vues par leur face inférieure. L'échantillon est représenté plus complètement, à échelle réduite, sur la planche XXXI.

Mines de Hongaỹ : Hatou, grande couche, grand banc de schistes. 


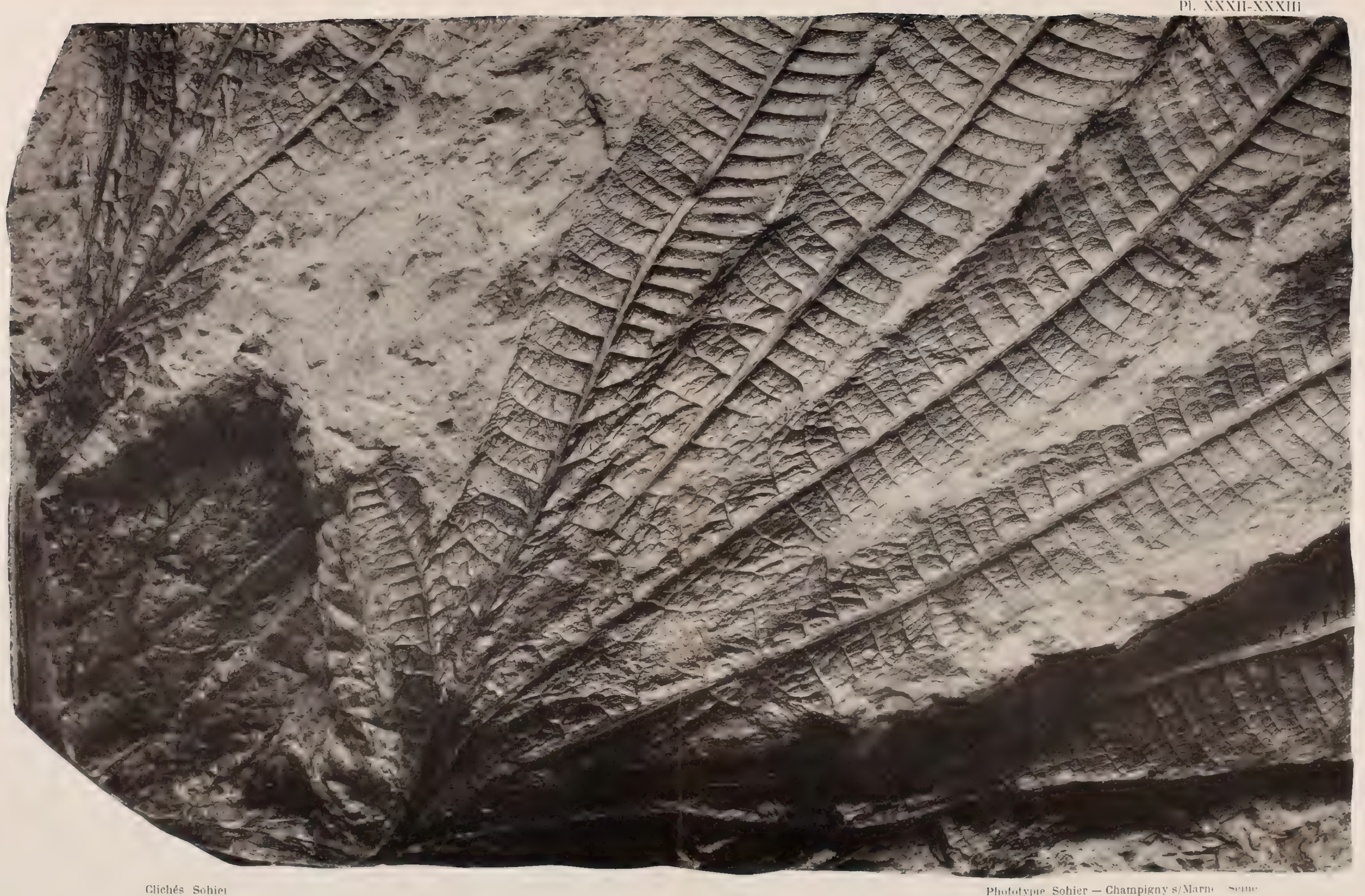




\section{PLANCHE XXXIV}




\section{PLANCHE XXXIV.}

\section{FXPLICATION DES FIGURES.}

Fis. 1. - Clathropteris platyphylla Goeppert (sp.). - Portion de fronde, en partie fertile, vue par la face inférieure. Sur le bord gauche de l'échantillon, on voit quelques pennes de Dictyophyllum Remauryi. Kébao.

Fig. la. - Portion de penne stérile du mème échantillon, grossie une fois et trois quarts.

Fig. Ib, lc. - Portions de pennes fertites du même échantillon, grossies deux fois et demie. 

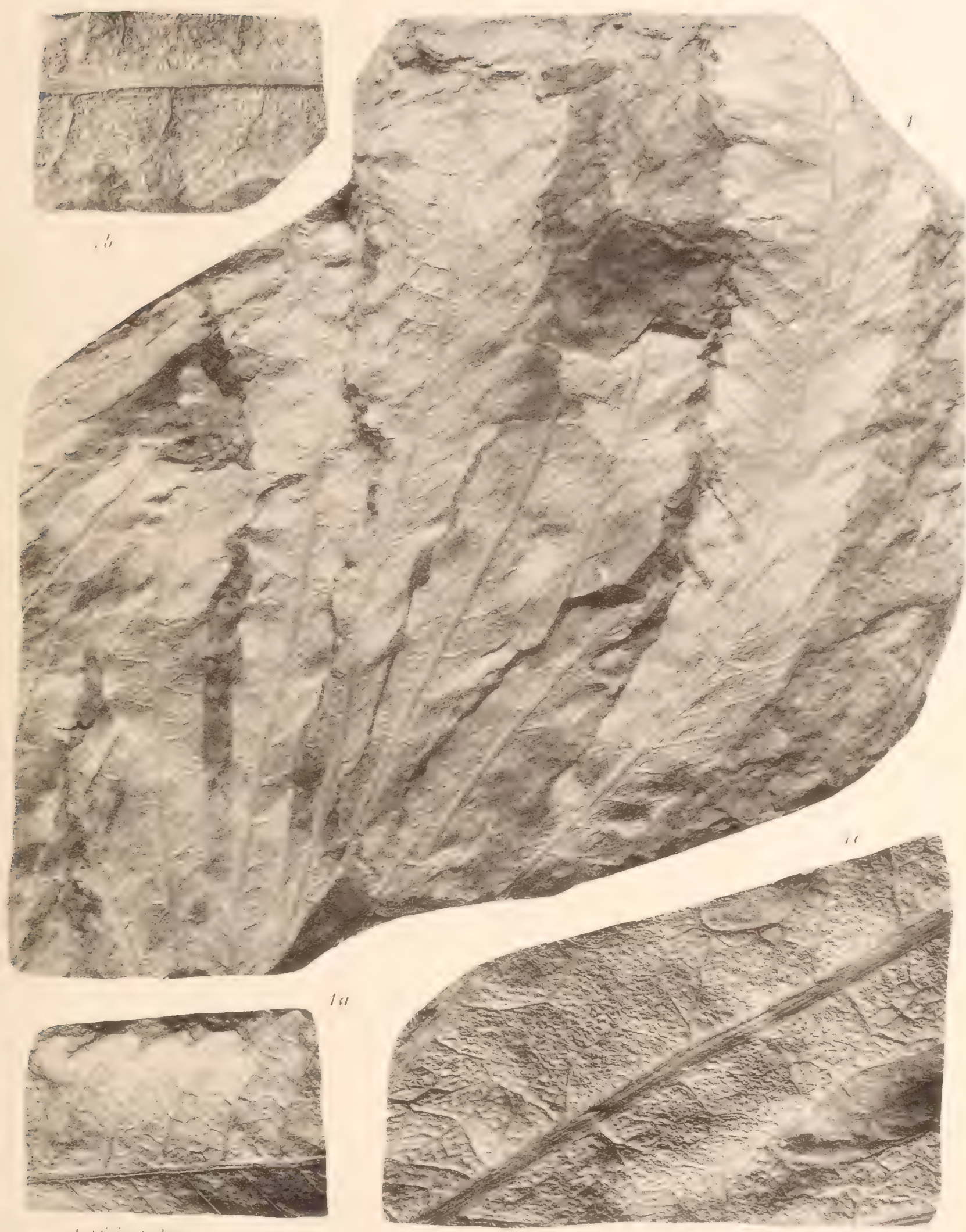

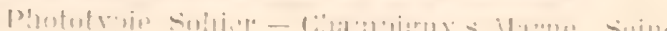




\section{PLANCHE XXXV}




\section{PLANCHE XXXV.}

\section{FXPLICATION DES FIGURES.}

Fig. I. - Spiropteris Schimper. - Fronde de Fougère enroulée en crosse.

Mines de Hongaỹ : Nagotna.

FI(i. 2. - Annulariopsis inopinata n. gen., n. sp. - Fragment de tige ou de rameau portant un verticille de feuilles.

Kébao, système supérieur, coụche $\mathrm{n}^{\circ} 2$, galerie M.

FIti. 3. - Annulariopsis inopinata n. gen., n. sp. - Fragment de verticille foliaire. Kébao, système supérieur, couche $\mathrm{n}^{\circ} 2$, galerie $M$.

Fig. 4. - Annulariopsis inopinata n.gen., n. sp. - Fragments de tiges ou de rameaux portant chacun un verticille de feuilles.

Kébao, système supérieur, couche $\mathrm{n}^{\circ} 2$, galerie $\mathbf{M}$.

FIG. 5. - Annulariopsis inopinata n. gen., n. sp. - Fragments de verticilles foliaires. Kébao, système supérieur, couche $\mathbf{n}^{\circ}$ 2, galerie M.

Fig. 6. - Annulariopsis inopinata n. gen., n. sp. - Fragment de tige ou de rameau portant un verticille de feuilles.

Kébao, système supérieur, couche $\mathbf{n}^{\circ}$ 2, galerie M.

Fig. 7. - Annulariopsis inopinata n. gen., n. sp. - Fragment de verticille foliaire. Hongaÿ, vallée orientale de l'OEuf, galerie Léonice.

FIg. 7 i. - Portion du même échantillon, grossie une fois et demie. 

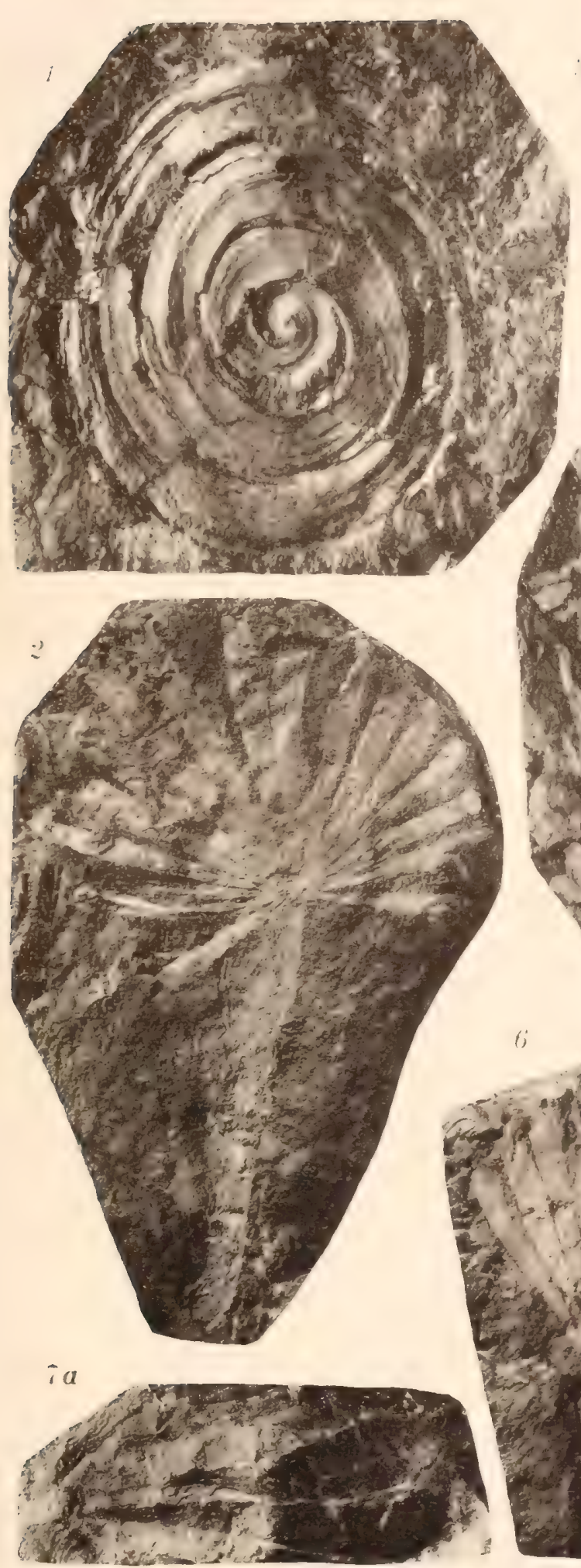

Clichés sohier.
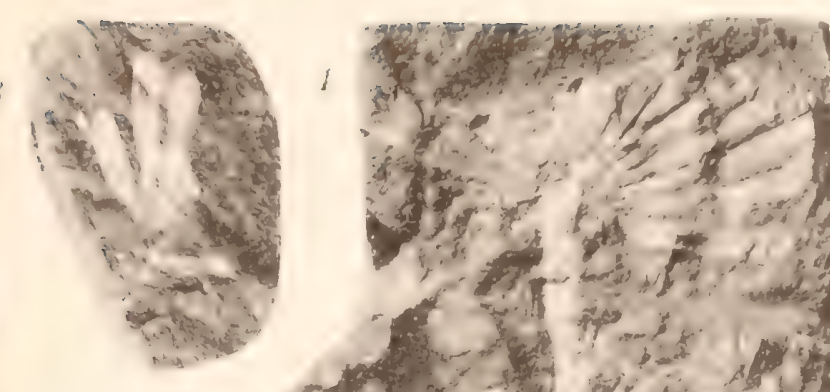

-

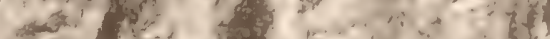
ac 50 a

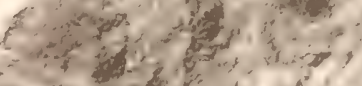
(5)

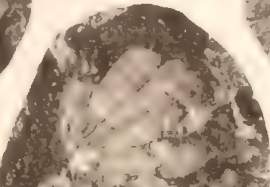

$(x-2)+2$ $(\cos )+3 x$ 1) (Wot ind $x^{2}+2$ (3) $+4$

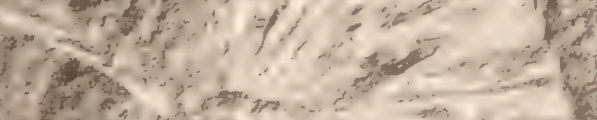
(f)

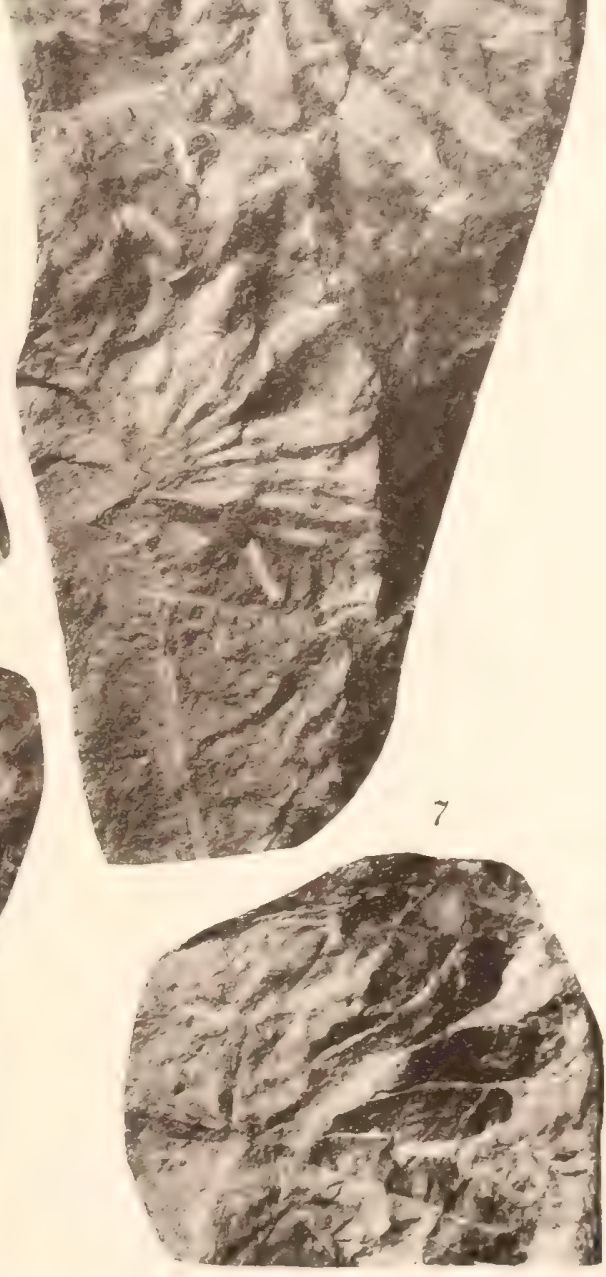

Phototvpie Sohier - Champigny-s/Marne (Seine) 



\section{PLANCHE XXXVI}




\section{PLANGHE XXXVI.}

\section{EXPLICATION DES FIGURES.}

FIG. 1 et 2. - Schizoneura Carrerei n. sp. - Fragments de tiges, avec plusieurs verticilles de feuilles.

Kébao, système inférieur, couche $n^{\circ} 1$. 
19. IXI1

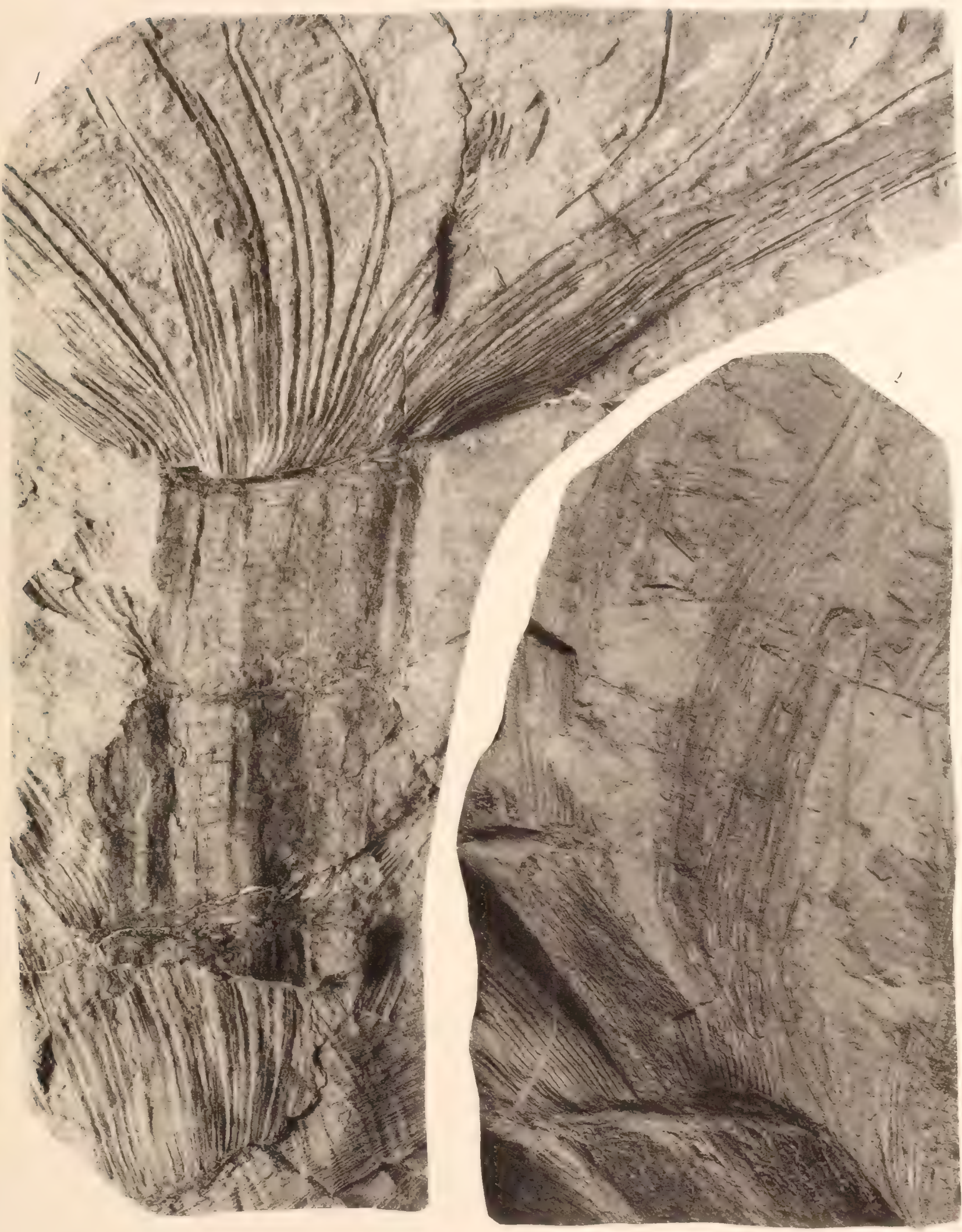

Clichés stohier

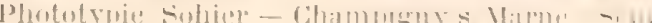





\section{PLANCHE XXXVII}




\section{PLANCHE XXXVII.}

\section{EXPLICATION DES FIGURES.}

Fig. - - Schizoneura Carrerei n. sp. - Fragments de tiges, avec plusieurs verticilles de feuilles.

Kébao, système inférieur, couche $\mathbf{n}^{\circ}$. 


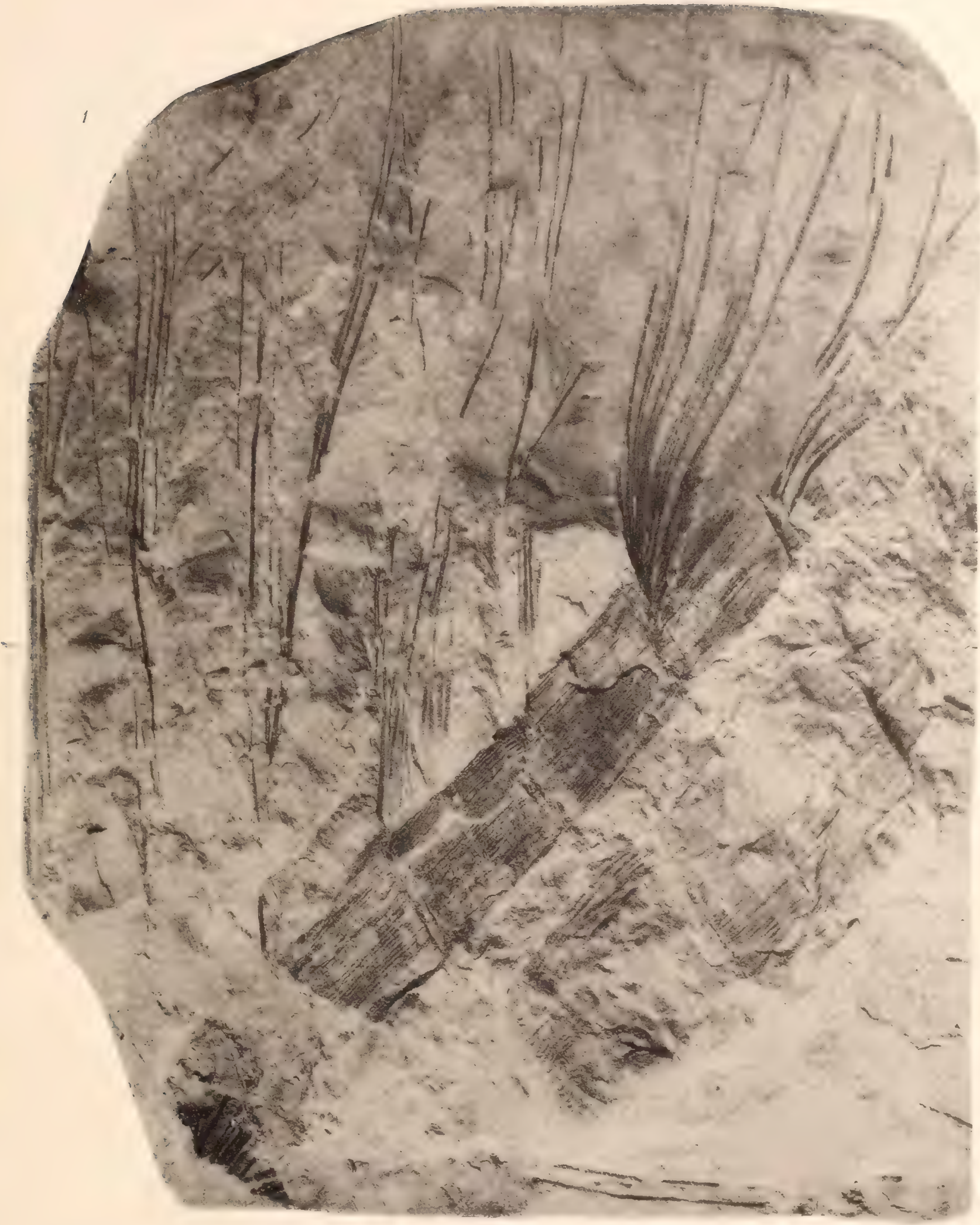


PLANGHE XXXVIII 


\section{PLANCHE XXXVIII.}

\section{EXPLICATION DES FIGURES.}

Fig. I. - Schizoneura Carrerei n. sp. - Fragment d'une grosse tige, dépouillée de ses feuilles, avec une partie du moule interne.

Mines de Hongaỹ : Hatou, grande couche.

FIG. 2. - Schizoneura Carrerei n.sp. - Fragment d'une tige, rompue à l'articulation, et offrant des cicatrices foliaires contiguës qui donnent aux portions de la surface de la tige comprises entre elles l'apparence de dents libres. Mines de Hongaỹ : Hatou, grande couche.

FIc. 3. - Schizoneura Carrerei n。 sp. - Empreinte d'un fragment de tige, avec cicatrices foliaires et deux cicatrices raméales.

Mines de Hongaÿ: Hatou, grande couche.

Fig. 1. - Schizoneura Garrerei n. sp.- Tige de petit diamètre, ou rameau, avec plusieurs verticilles de feuilles.

Hongay̆, mine de Carrère, couche Marmottan.

Fig. 5. - Schizoneura Carrerei n. sp. - Moule interne d'une tige.

Mines de Hongaỹ : Hatou, grande couche.

FIG. 6. - Schizoneura Carrerei n. sp. - Empreinte d'un fragment de tige, montrant quelques restes de feuilles, encore attachées à l'articulation, et la surface interne de l'entrenœud supérieur.

Hongaỹ, mine de Carrère, couche Marmottan.

Fic. 6 a. - Portion du même échantillon, grossie une fois et demie.

FIrs. 7. - schizoneura Carrerei n. sp. - Fragment de moule interne d'une tige, et rameau.

Mines de Hongaÿ : Hatou, grande couche.

Fig. 8. - Schizoneura Carrerei n. sp. - Articulation vue à plat, montrant la base des feuilles et le diaphragme interne (échantillon figuré Bull. Soc. Géol. de France, $3^{\circ}$ série, t. XIV, pl. XXIV, fig. 1).

Bassin de Hongaÿ. 
PI. XXXVIII

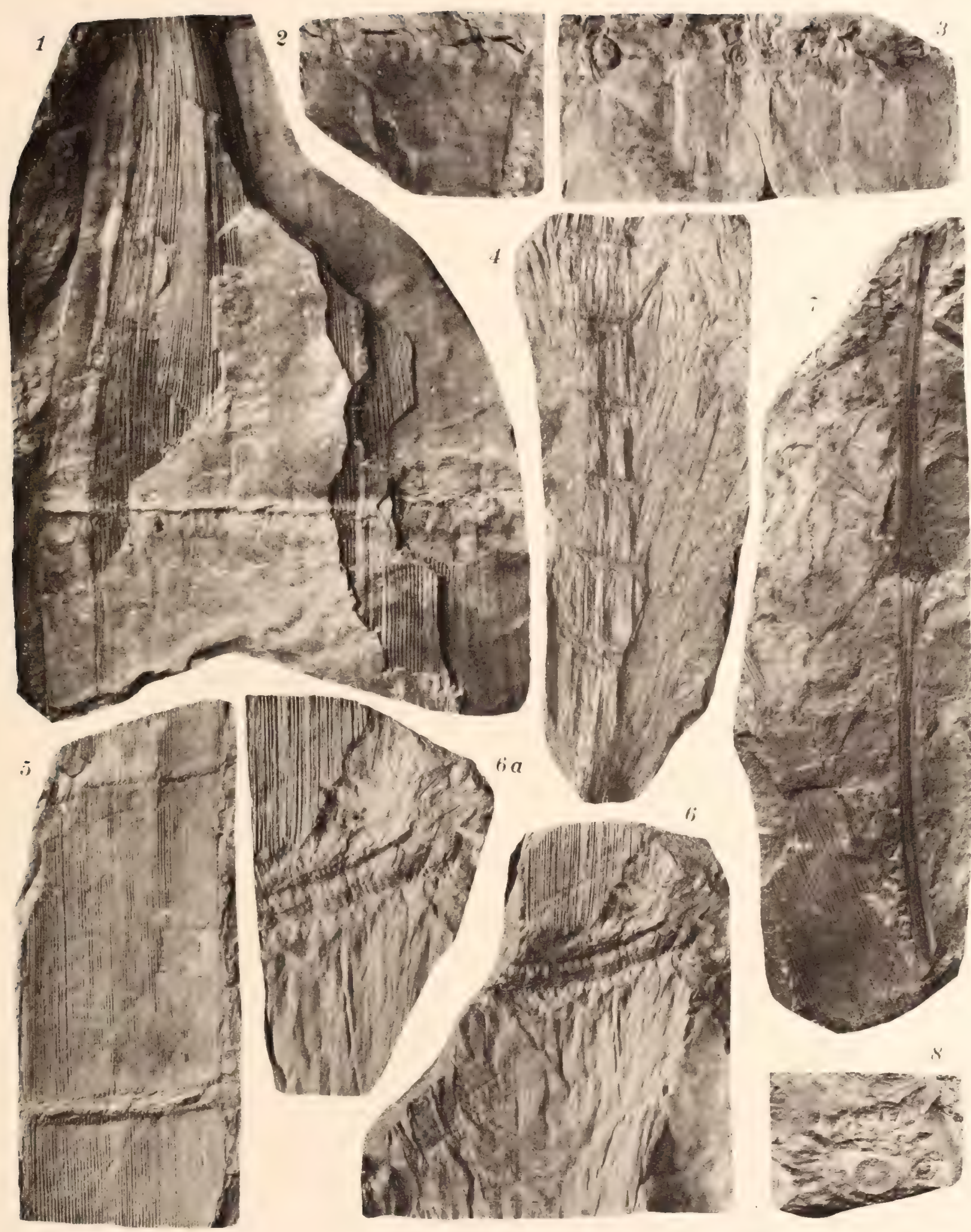

Clichés Sohier.

Phototypie Sohier - Champigny-s/Marne (Seine) 


\section{PLANCHE XXXIX}




\section{PLANCHE XXXIX.}

\section{EXPLICATION DES FIGURES}

Fig. 1. - Equisetum Sarrani n. sp. - Fragment de tige.

Hongaÿ, vallée orientale de l'OEuf, couche près d'une petite île.

FIG. 1a. - Portion du mème échantillon, grossie deux fois, montrant les ponctuations des côtes.

Frg. 2. - Equisetum Sarrani n. sp. - Fragment de tige avec articulation et base d'une gaine foliaire.

Mines de Hongaÿ: Hatou, grande couche, grand banc de schistes.

Fig. 2 a. - Portion du même échantillon, grossie trois fois, montrant les ponctuations des côtes.

Fig. 3. - Equisetum Sarrani n. sp. - Fragment de tige avec articulation et base d'une gaine foliaire.

Mines de Hongai": Hatou, grande couche, grand banc de schistes.

Fig. 4. - Equisetum Sarrani n. sp. - Épi de fructification.

Mines de Hongaỹ : Hatou, grande couche, grand banc de schistes.

Fig. 4a. - Base du même épi, grossie deux fois.

Fig. 4b. - Portion du même épi, grossie quatre fois.

Fig. 5. - Equisetum Sarrani n. sp. - Fragment de tige (ou de rhizôme) avec une racine attachée et deux cicatrices d'insertion de racines ou de rameaux.

Hongaÿ, vallée orientale de l'OEuf, couche près d'une petite île.

FIG. 5 a. - Portion du même échantillon, grossie deux fois.

Fig. 6. - Equisetum Sarrani n. sp. - Fragment de moule interne.

Mines de Hongay: Hatou, grande couche, grand banc de schistes.

FIG. 7. - Equisetum Sarrani n. sp. — Fragment de rameau.

Hongaỹ, vallée orientale de l'OEuf, couche près d'une petite île.

FIG. 7 a. - Portion du même échantillon, grossie une fois et demie, montrant les ponctuations de la surface.

FIG. 8 et 9. - Equisetum Sarrani n. sp. - Empreintes de rameaux.

Mines de Hongaÿ : Hatou, grande couche, grand banc de schistes.

Fig. 8 a et 9 a. - Portions des mèmes échantillons, grossies trois fois et demie.

FIg. 10 et 11. - Equisetum Sarrani n. sp. - Empreintes de rameaux montrant leur extrémité supérieure.

Mines de Hongaỹ: Hatou, grande couche, grand banc de schistes.

Fig. 12. - Equisetum Sarrani n. sp. - Empreinte de la section transversale d'un rameau rompu à une articulation.

Mines de Hongay: Hatou, grande couche, grand banc de schistes.

Fig. 13. - Equisetum Sarrani n. sp. - Section transversale d'un rameau rompu à une articulation.

Mines de Hongay : Hatou, grande couche, grand banc de schistes.

FIG. 13 a. - Le même échantillon, grossi trois fois 

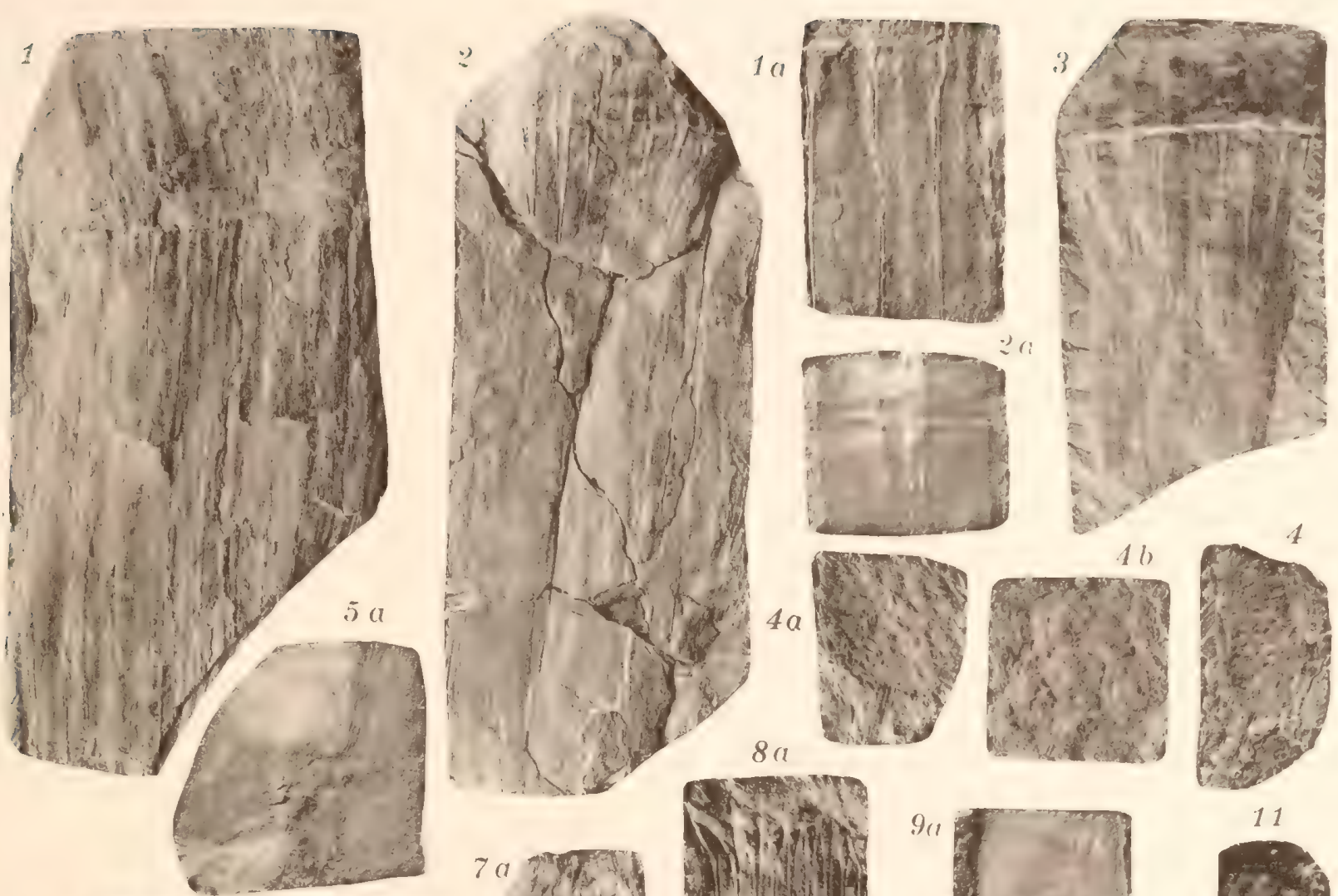

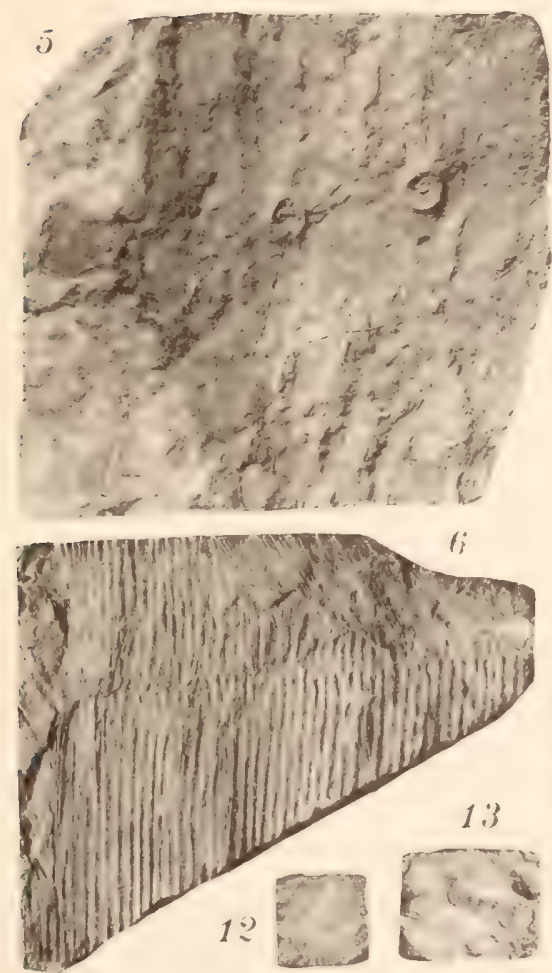

Clichés Sohier.

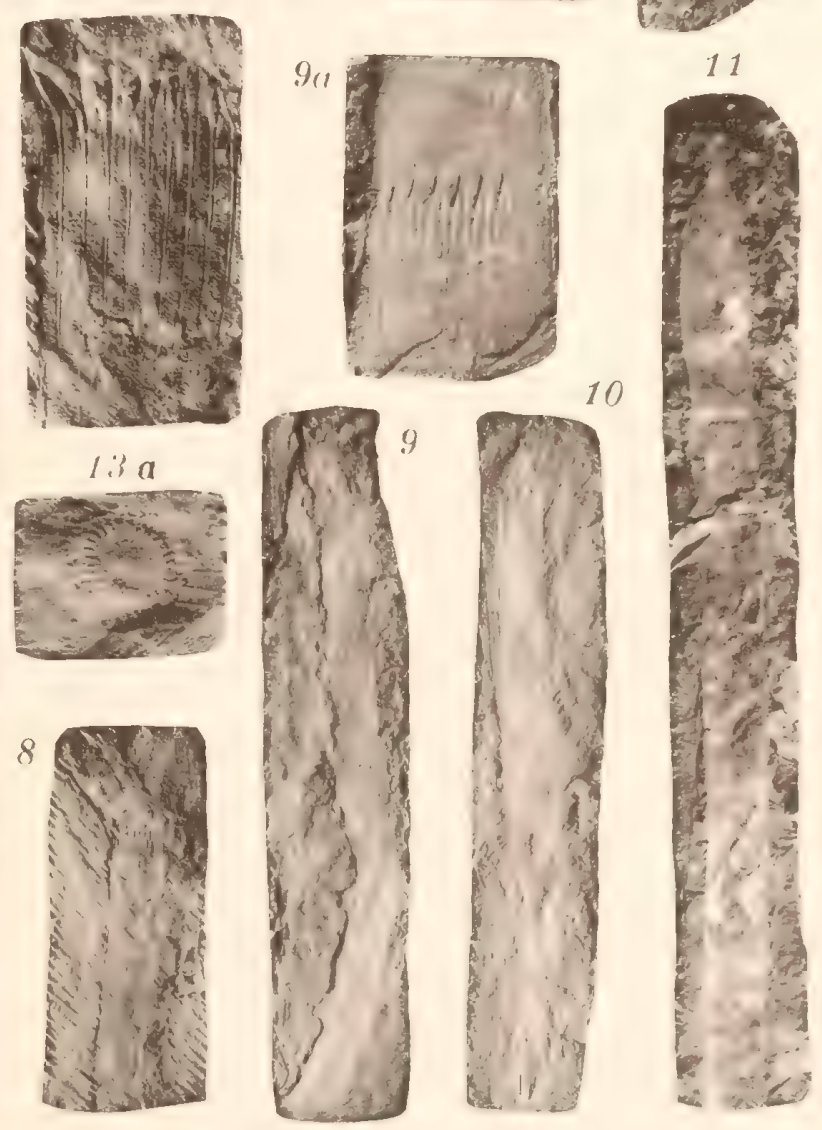

Phototypie Sohier -. Champigny-s/Marne (Seine) 

PLANGHE XL

10. 


\section{PLANGHE XL.}

\section{EXPLICATION DES FIGURES.}

Fig. 1 et 2. - Nœggerathiopsis Hislopi Bunbury (sp.). - Feuilles détachées (forme étroite).

Mines de Hongay: Hatou, au mur de la grande couche.

Fig. 3. - Nœggerathiopsis Hislopi Bunbury (sp.). - Feuille détachée (forme étroite, rétrécie à la base), (figurée Annales des Mines, $8^{\text {e }}$ série, t. II, pl. XII, fig. 11 ).

Bassin de Hongaỹ, monticule rive gauche en aval de Claireville.

FiG. 4. - Nœggerathiopsis Hislopi Bunbury (sp.). - Partie inférieure d'une fenille (forme rétrécie à la base).

Kébao, puits Lanessan.

FIG. 5. - Nœggerathiopsis Hislopi Bunbury (sp.). - Feuille incomplète (forme large). Kébao.

Fig. 5 a. - - Portion du même échantillon, grossie deux fois.

Fig. 6. - Nœggerathiopis Hislopi Bunbury (sp.). - Fragment d'une feuille attaquée par un Champignon.

Hongaỹ, vallée orientale de l'OEuf, galerie Léonice.

Fig. 7. - Rameau, appartenant probablement au Noggerathiopsis Hislopi.

Hongaÿ, au toit de la couche Marguerite.

Fig. 7a. - Portion du même échantillon, grossie une fois et demie, montrant les cicatrices foliaires.

Fig. 8. - Empreinte d'un fragment de rameau, appartenant probablement au Noeggerathiopsis Hislopi (échantillon figuré Annales des Mines, $8^{\circ}$ série, t. II, pl. XI, fig. 14).

Kébao.

Fig. 8 a. - Portion du même échantillon, grossie une fois et demie.

Fig. 9. - Fragment de rameau, appartenant peut-être au Noggerathiopsis Hislopi. Kébao.

Fig. 9 a. - Portion du même échantillon, grossie une fois et demie. 


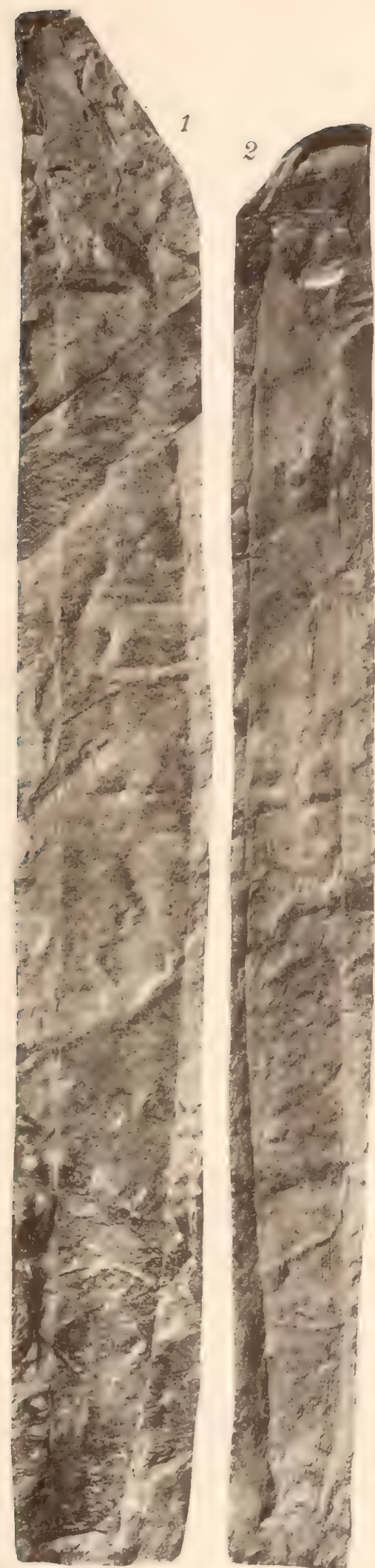

Clichés Sohier.
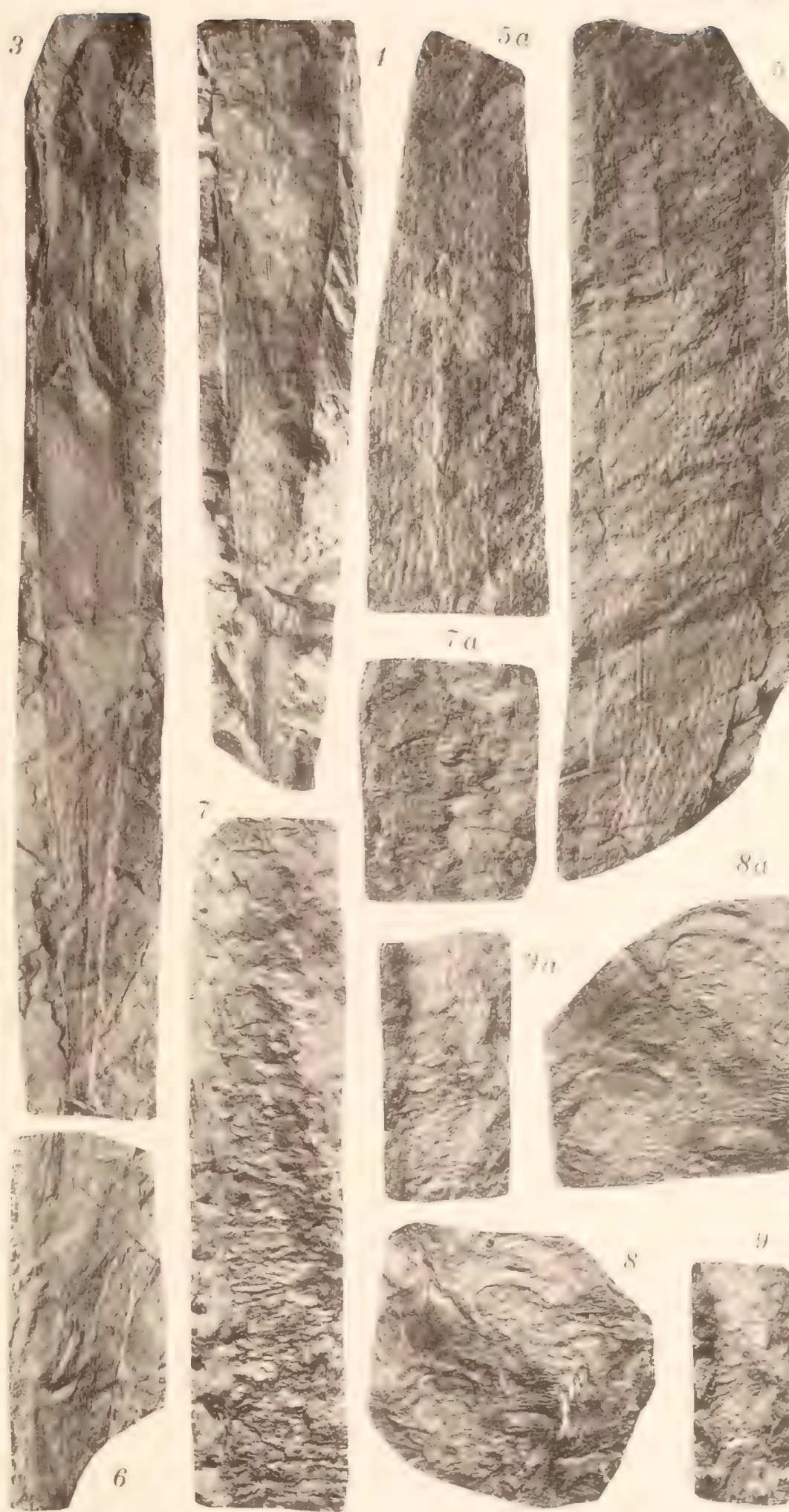

2.
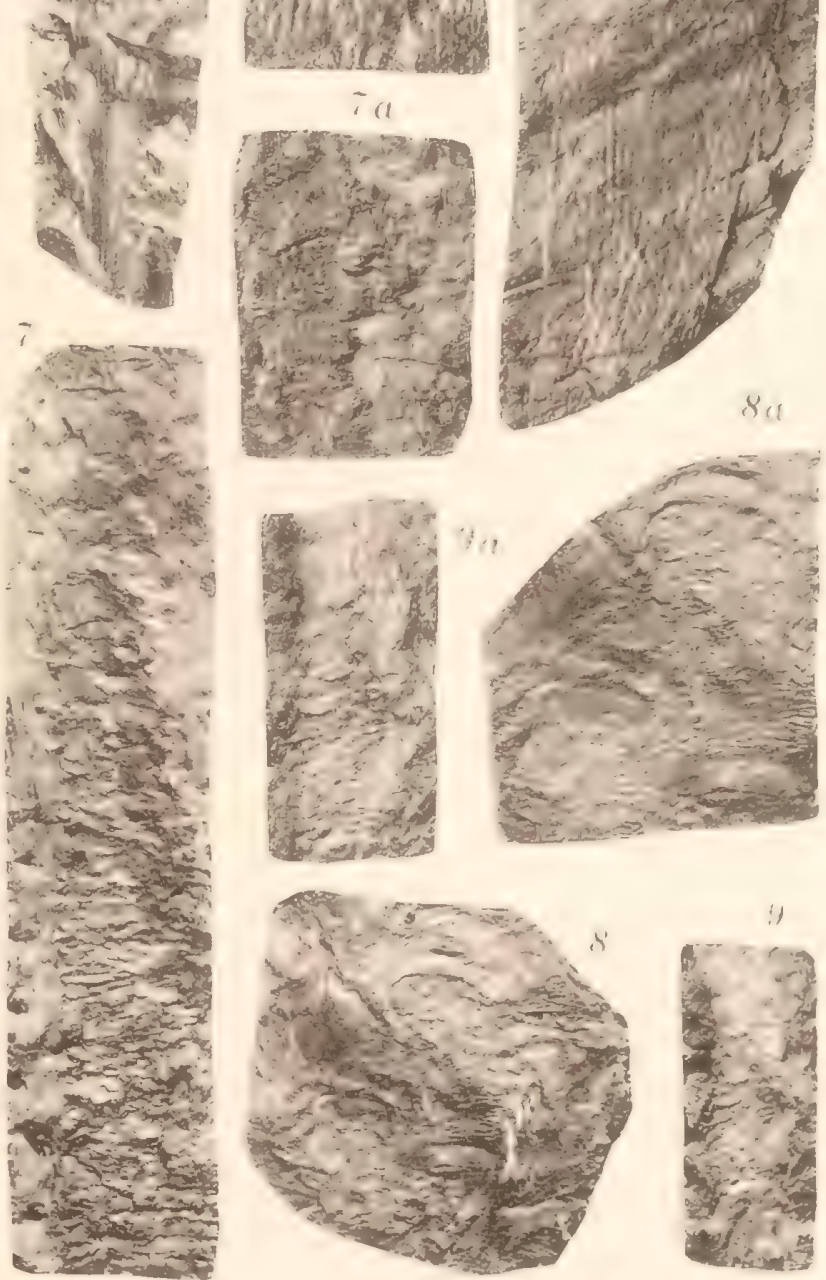

Phototypie Sohier - Champigny-s/Marne (Seine) 



\section{PLANCHE XLI}




\section{PLANCHE XLI.}

\section{EXPLICATION DES FIGURES.}

Fig. I. - Gycadites Saladini Zeiller. - Fronde incomplète.

Mines de Dongtrieu : village de Cokinh, périmètre Émile, au mur de la couche D.

F1G. 2. - Gycadites Saladini ZeILler. - Fragment de fronde.

Mines de Dongtrieu : village de Cokinh, périmètre Émile, au mur de la couche D.

FIgr. 2a. - Portion du même échantillon, grossie une fois et demie.

Fig. 3. - Cycadites Saladini Zeiller. - Partie inférieure d'une fronde.

Mines de Dongtrieu : village de' Cokinh, périmètre Émile, au mur de la couche D.

Fig. 4. - Cycadites Saladini ZeIller. - Fragments de frondes.

Mines de Hongaÿ, découvert de Hatou.

Fıg. 4a. - Portion du même échantillon, grossie une fois et demie. 

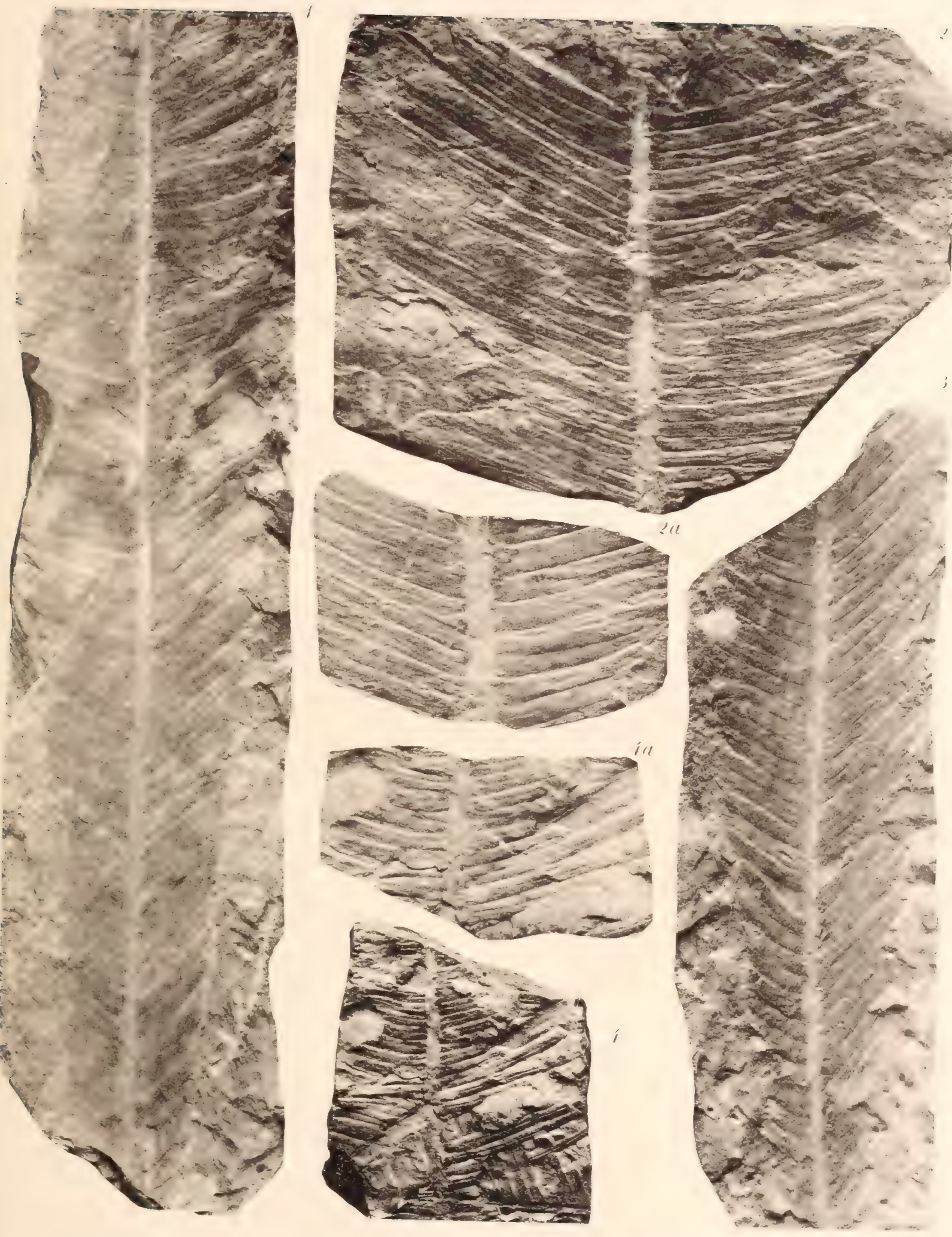

tilichés sohier 
. 
PLANCHE XLII 


\section{PLANCHE XLII.}

\section{EXPLICATION DES FIGURES.}

FiG. 1. - Podozamites distans Presl (sp.). - Fronde incomplète, à folioles en partie détachées ou dérangées de leur position normale.

Mines de Dongtrieu.

Fig. 2. - Podozamites distans Presl (sp.). - Foliole détachée (figurée Annales des Mines, $8^{\circ}$ série, t. II, pl. XI, fig. 2 ).

lìle de Hongaÿ.

Fig. 3. - Podozamites distans Prest (sp.). - Folioles détachées.

Mines de Dongtrieu.

Fig. 4. - Podozamites distans Press (sp.). - Fragment de fronde (figuré Bull. Soc. Géol. de France, $3^{\text {e }}$ sér., t. XIV, pl. XXIV, fig. 8).

Bassin de Hongaỹ.

Fig. 5 et 6. - Podozamites Schenki Herr. - Portions de frondes.

Mines de Hongaỹ, découvert de Hatou. 


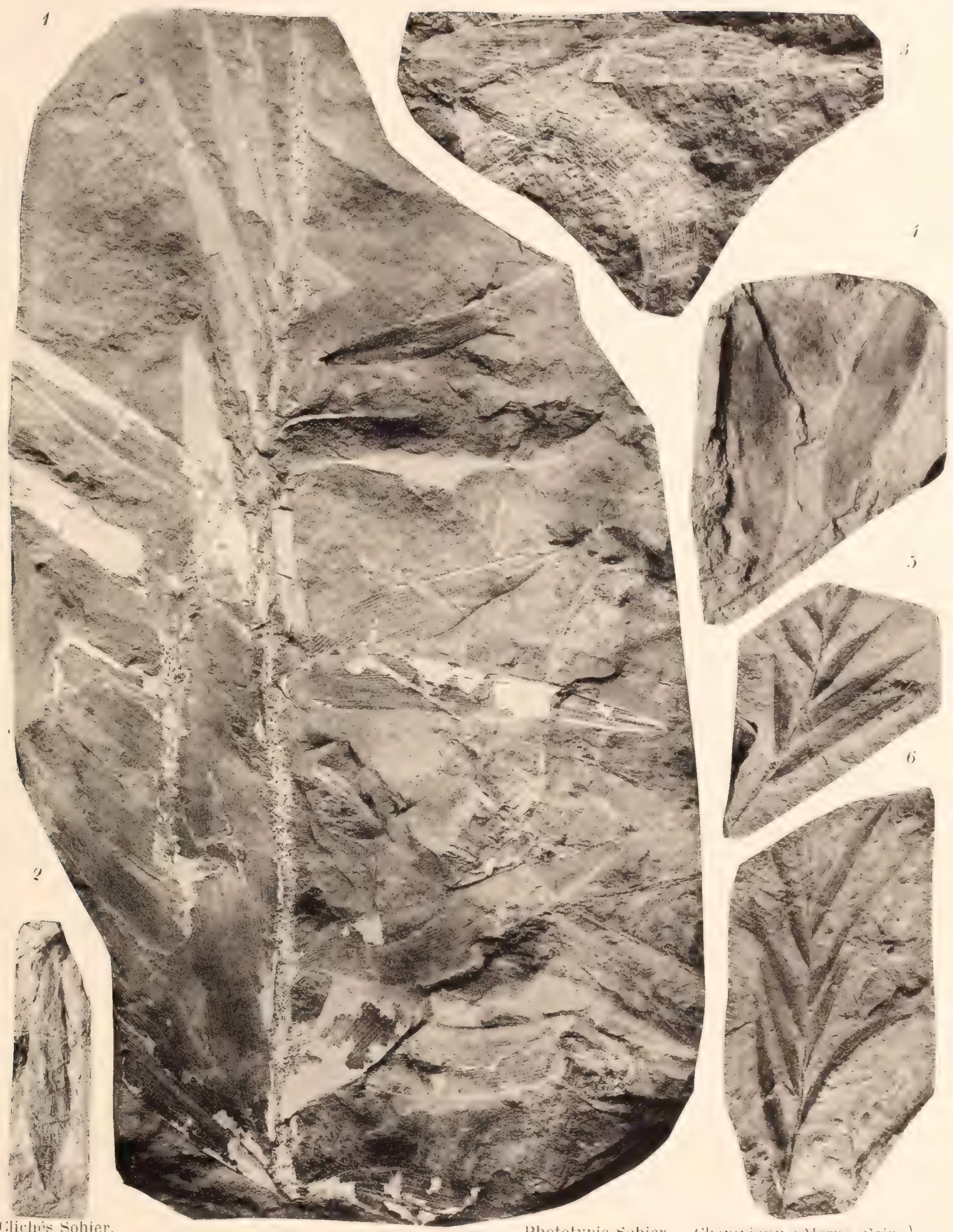

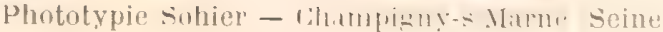




\section{PLANCHE XLIII}




\section{PLANCHE XLIII.}

\section{EXPLICATION DES FIGURES.}

FIG. 1. - Otozamites indosinensis n. sp. - Fragment de fronde. Mines de Hongä.

FIG. 1a. - Portion du même échantillon, grossie deux fois.

Fig. 2. - Otozamites rarinervis Feistmantel. - Fronde incomplète (figuríe Annales des Mines, $8^{\text {e }}$ série, t. II, pl. XII, fig. 7).

Hongaÿ, mine Jauréguiberry.

líg. 2a. - Portion du même échantillon, grossie deux fois.

Fig. 3. Zamites truncatus n. sp. - Fragment de fronde.

Hongaỹ, vallée orientale de l'OEuf, galerie Léonice.

FIg. 4. - Zamites truncatus n. sp. - Foliole détachée.

Hongaỹ, vallée orientale de l'OEuf, galeric Léonice.

Fig. 4a. - Le même échantillon, grossi une fois et demie.

FIG. 5 et 6 . - Zamites truncatus n. sp. - Folioles détachées.

Hongaỹ, vallée orientale de l’OEuf, galerie Léonice.

Fig. 6. - Pterophyllum (Anomozamites) schenki ZellLer. - Portion de fronde (figurée Bull. Soc. Géol. de Fr., 3e sér., t. XIV, pl. XXIV, fig. 9).

Bassin de Hongä̈.

Fig. 7 a. - Portion du même échantillon, grossie deux fois.

Fig, 8. - Pterophyllum (Anomozamites) inconstans Brauv (sp.). - Fragment de fronde.

Hongaÿ, vallée orientale de l’(OEf, galerie Léonice. 

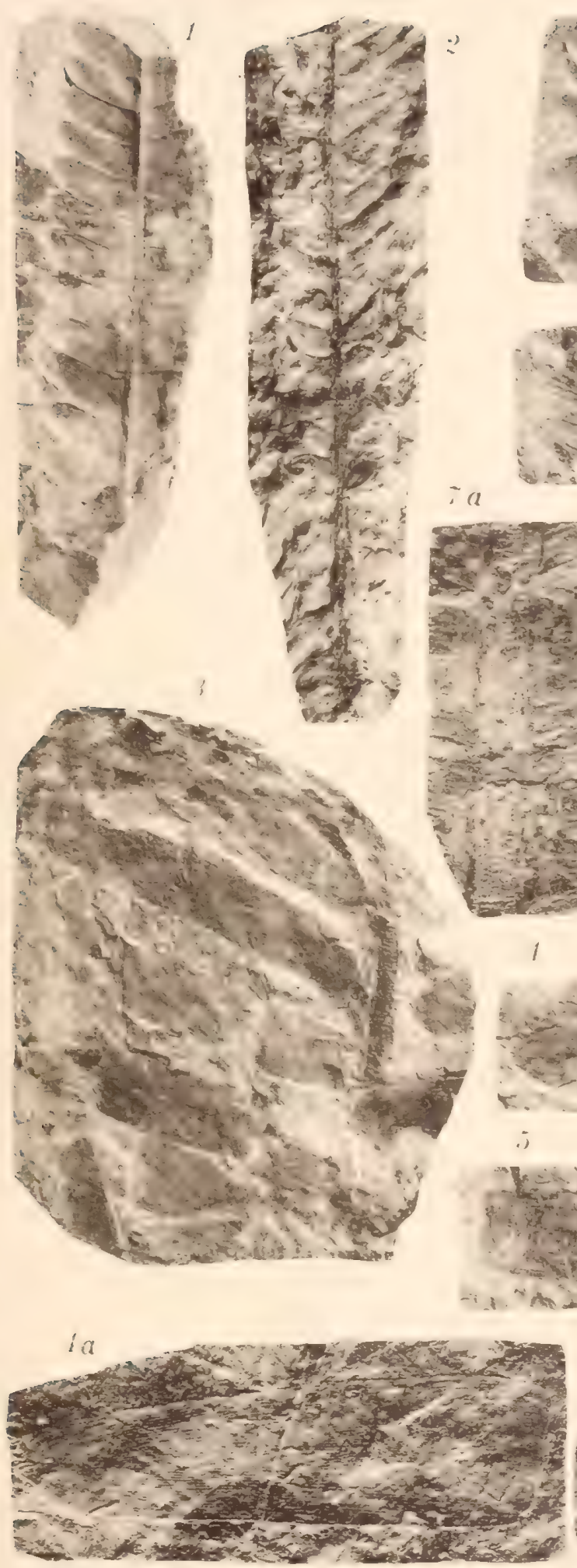

Clichés Sohier.
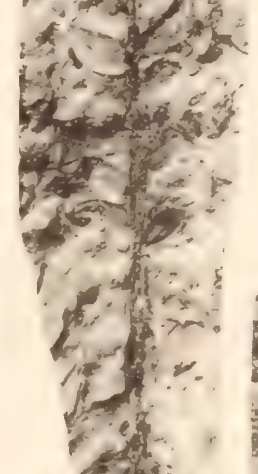

$\because a$
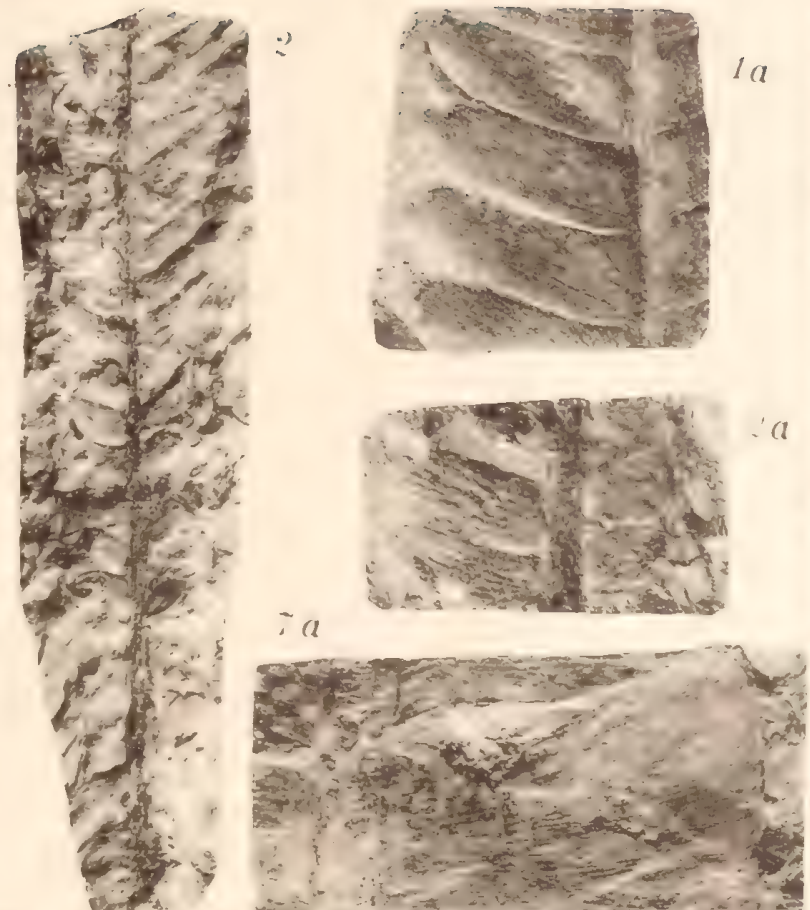

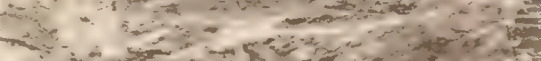

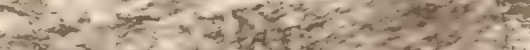

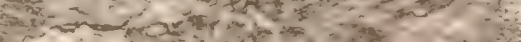
Le $x^{2}=2 x+2$

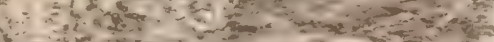
$(-3)^{3}=-20 x^{2}$
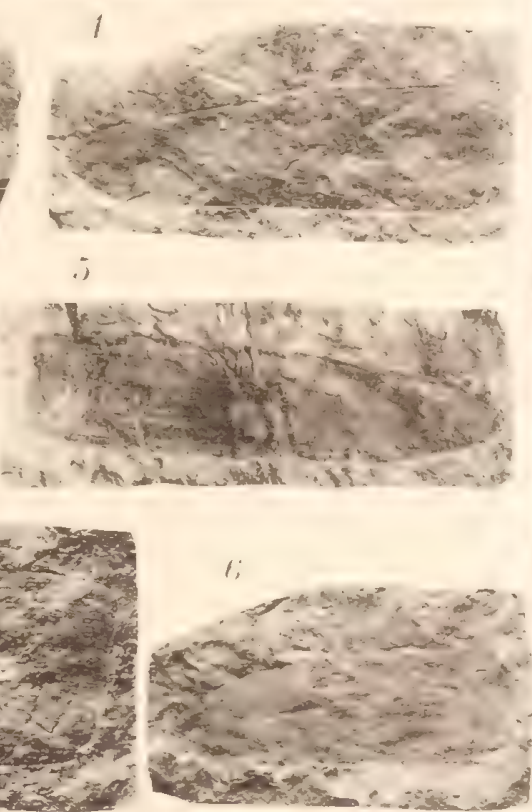

Pholotypie Sohier

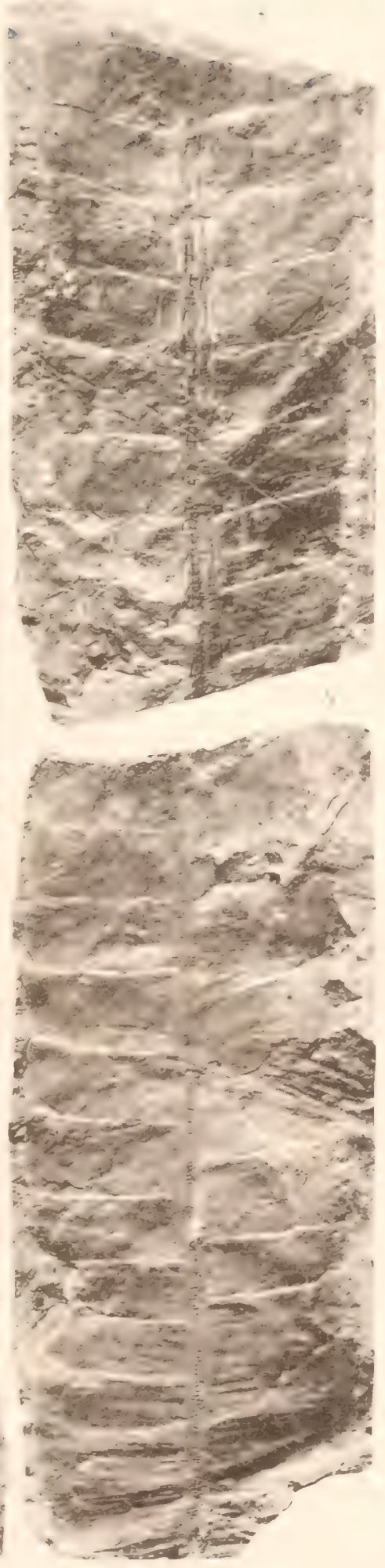

Chanpiguy-s/Marne (Seine) 



\section{PLANCHE XLIV}




\section{PLANCHE XLIV.}

\section{EXPLICATION DES FIGURES}

Fig. 1. - Pterophyllum (Anomozamites) inconstans Braun (sp.). - Fragment de fronde; à gauche et vers le bas de l'échantillon, une écaille de Cycatdinée (Cycadolepis corrugata n. sp.).

Hongaỹ, vallée orientale de l'OEuf, galerie Léonice.

FIG. 1a. - Portion du même échantillon, grossie deux fois.

Fig. 2. - Pterophyllum (Anomozamites) inconstans Brauv (sp.). - Fronde presque complète, à limbe entier dans sa région inférieure.

Hongaỹ, vallée orientale de l'OEuf, galerie Léonice.

Fig. 3. - Pterophyllum (Anomozamites) inconstans Braun (sp.). - Fronde incomplète.

Hongay, île du Sommet Buisson, tranchée en avant de la galerie Jean.

Fig. 4. - Pterophyllum (Anomozamites) inconstans Braun (sp.). - Portion de fronde.

Hongaỹ, île du Sommet Buisson, tranchée en avant de la gaterie Jean.

FIG. 4 a. - Portion du même échantillon, grossie une fois et deux tiers.

FIfr 5. - Pterophyllum (Anomozamites) inconstans Braun (sp...- Portion inférieure d'une fronde.

Mines de Hongaỹ, découvert de Hatou. 
PI. XLIV

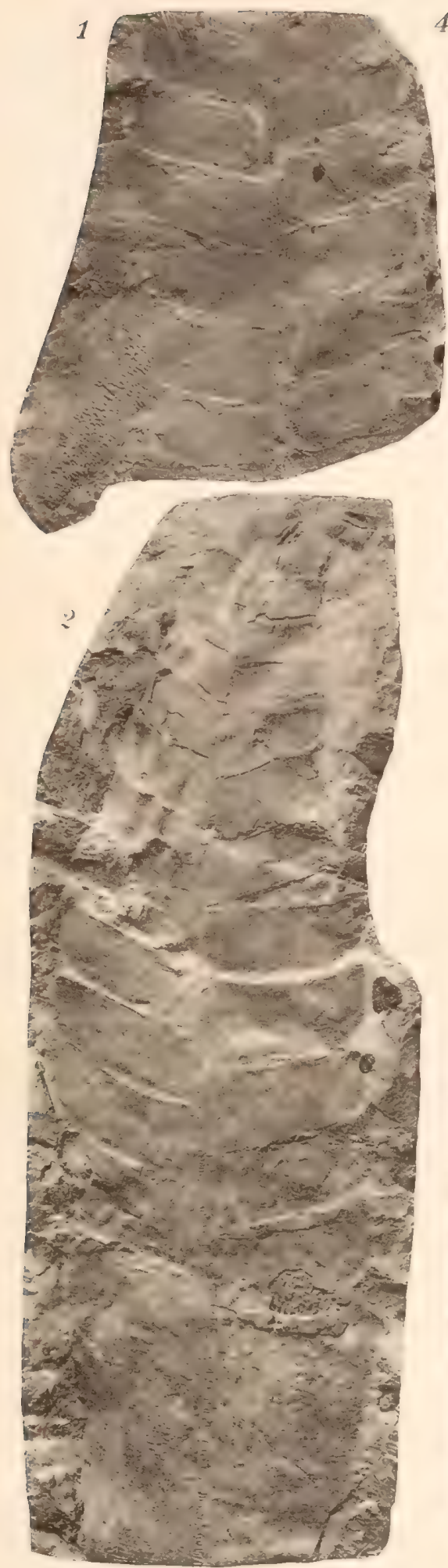

Clichés Sohier.
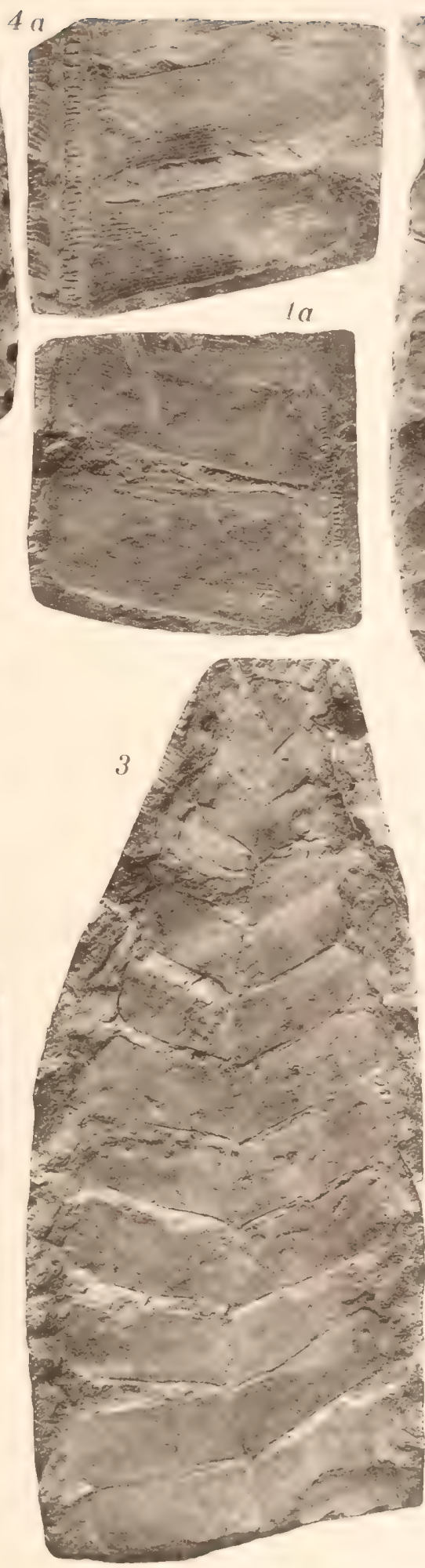

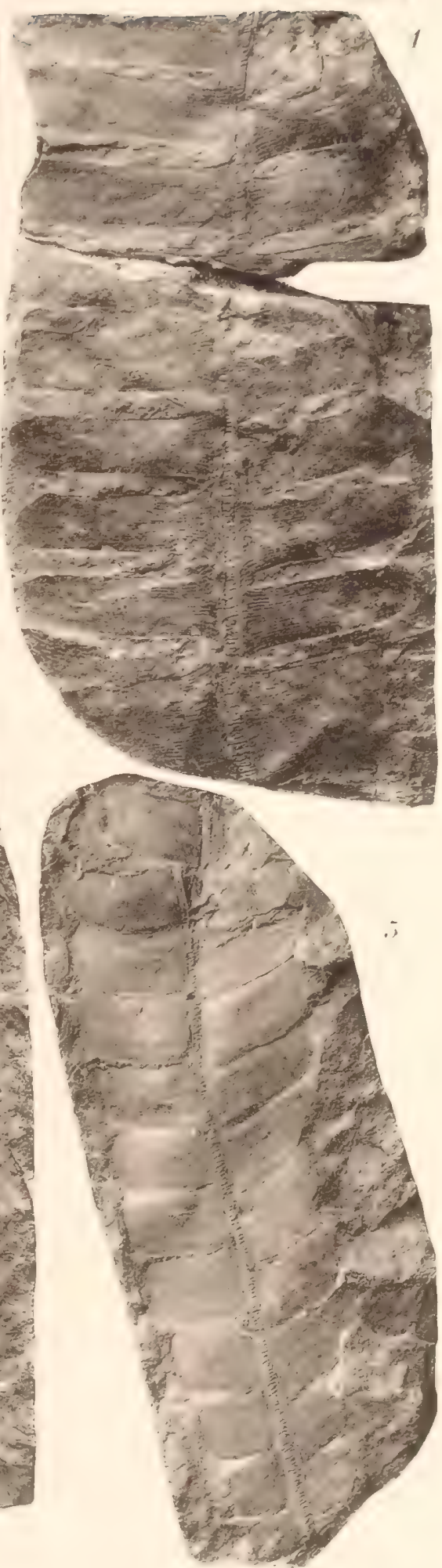

Phototypie Sohier - Champign--s/Marne (Seine) 

PLANCHE XLV 


\section{PLANCHE XLV.}

EXPLICATION DES FIGURES.

FIG. 1. - Pterophyllum Münsteri Presl (sp.). - Fragment de fronde.

Mines de Hongay : Nagotna, au toit de la couche Bavier.

Fig. 2. - Pterophyllum Münsteri Presl (sp.)._- Fronde incomplète.

Hongay̆, mine de Carrère, au toit de la couche Bavier.

Fig. 3. - Pterophyllum Münsteri Presl (sp.). — Fronde incomplète.

Bassin de Hongaỳ.

FIG. 3 a. - Portion du même échantillon, grossie deux fois.

FIG. 4. - Pterophyllum Münsteri Presl (sp.). - Frondes provenant d'un jeune pied.

Mines de Hongaỹ, découvert de Hatou (collections de géologie du Muséum d'histoire naturelle de Paris).

Fig. 4a. - Portion de fronde du même échantitlon, grossie deux fois.

Fig. 5. - Pterophyllum Münsteri Presl (sp.). - Portions de frondes.

Mines de Dongtrieu : village de Cokinh, périmètre Émile, au mur de la couche B.

FIG. 5a. - Portion de fronde du même échantillon, grossie deux fois. 
PI. XLV

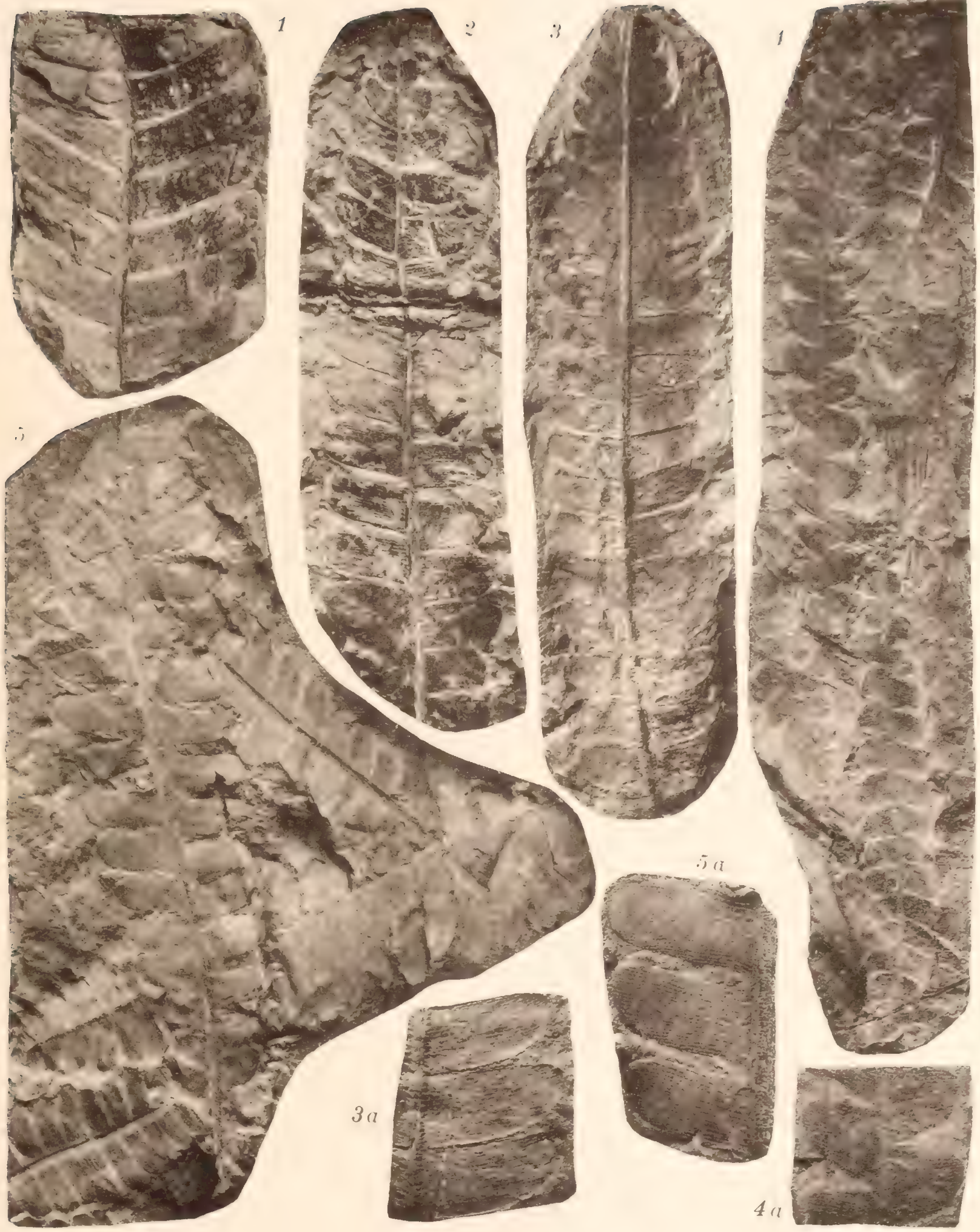

Clichés Sohier.

Phototypie Sohier - Champigny-s/Marne (Seine) 
PLANCHE XLVI

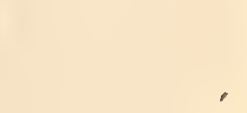




\section{PLANCHE XLVI.}

\section{EXPLICATION DES FIGURES.}

Fig. 1. - Pterophyllum Portali n. sp. - Fronde complete.

Kébao, mine Rémaury, au toit de la couche Q.

FIg. 2. - Pterophyllum Portali n. sp. - Fragment de fronde.

Kébao, puits Lanessan, au mur de la couche Descenderie.

Fig. 3. - Pterophyllum Portalí n. sp. - Fronde complète.

Kébao, couche $G$.

Fig. 4. - Pterophyllum Portali n. sp. - Portion inférieure d'une fronde. Kébao, couche G.

FIg. 4 a. - Portion du même échantillon, grossie une fois et demie.

Fig. 5. - Pterophyllum Portali n. sp. - Portion inférieure d'une fronde. Kébao, système supérieur, couche $n^{0} 2$, galerie $M$.

Fig. 5a. - Portion du même échantillon, grossie une fois et demie. 


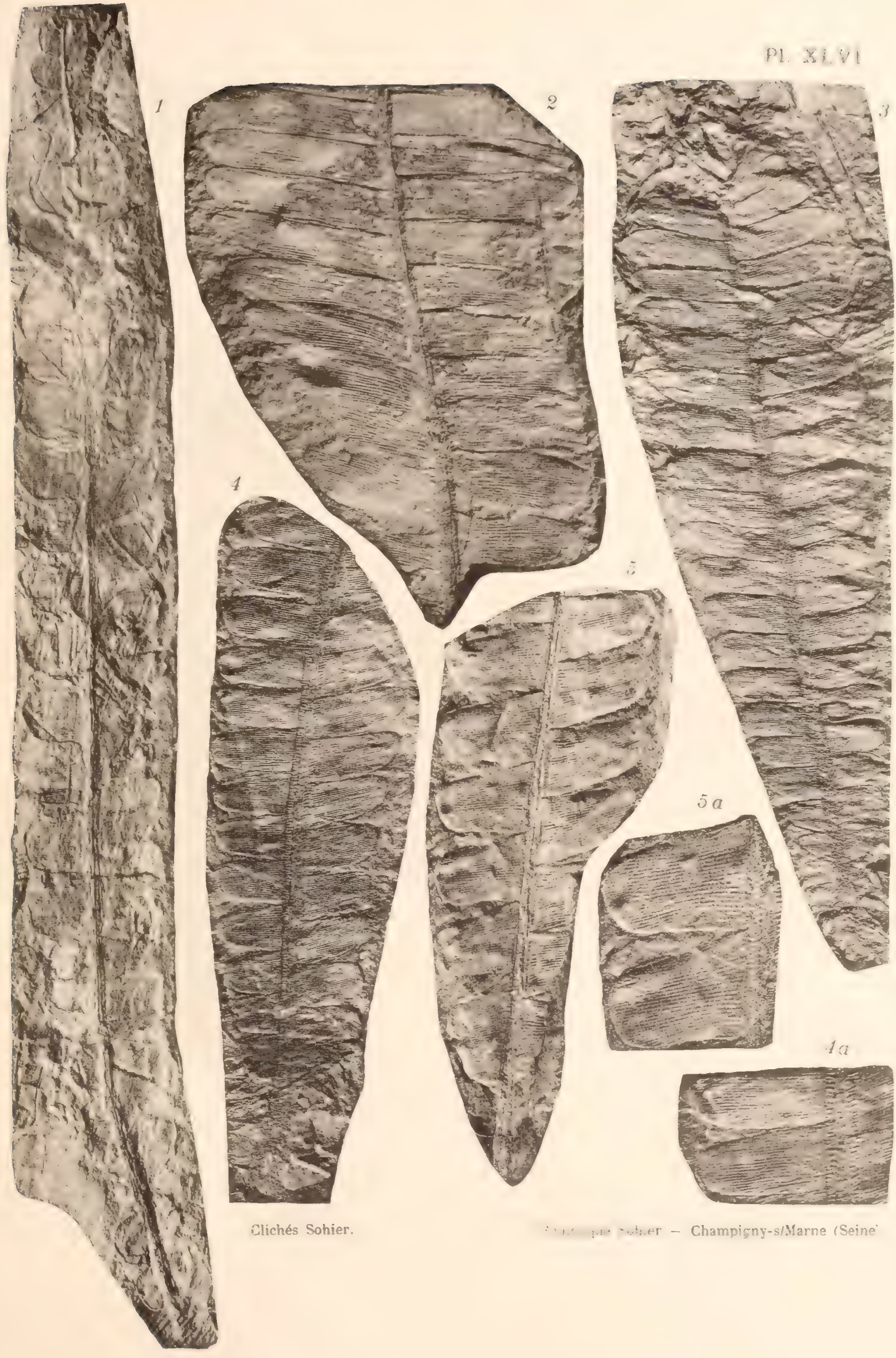





\section{PLANGHE XLVII}




\section{PLANCHE XLVII.}

\section{EXPLICATION DES FIGURES.}

Fig. 1a. - Pterophyllum Tietzei Schenk. - Portion inférieure d'une fronde, réduite aux deux cinquièmes de la grandeur naturelle.

Kébao, mine de Caï-Dai.

Fig. I et l'. - Portions du même ćchantilion; grandeur naturelle. 
P. XLVII.
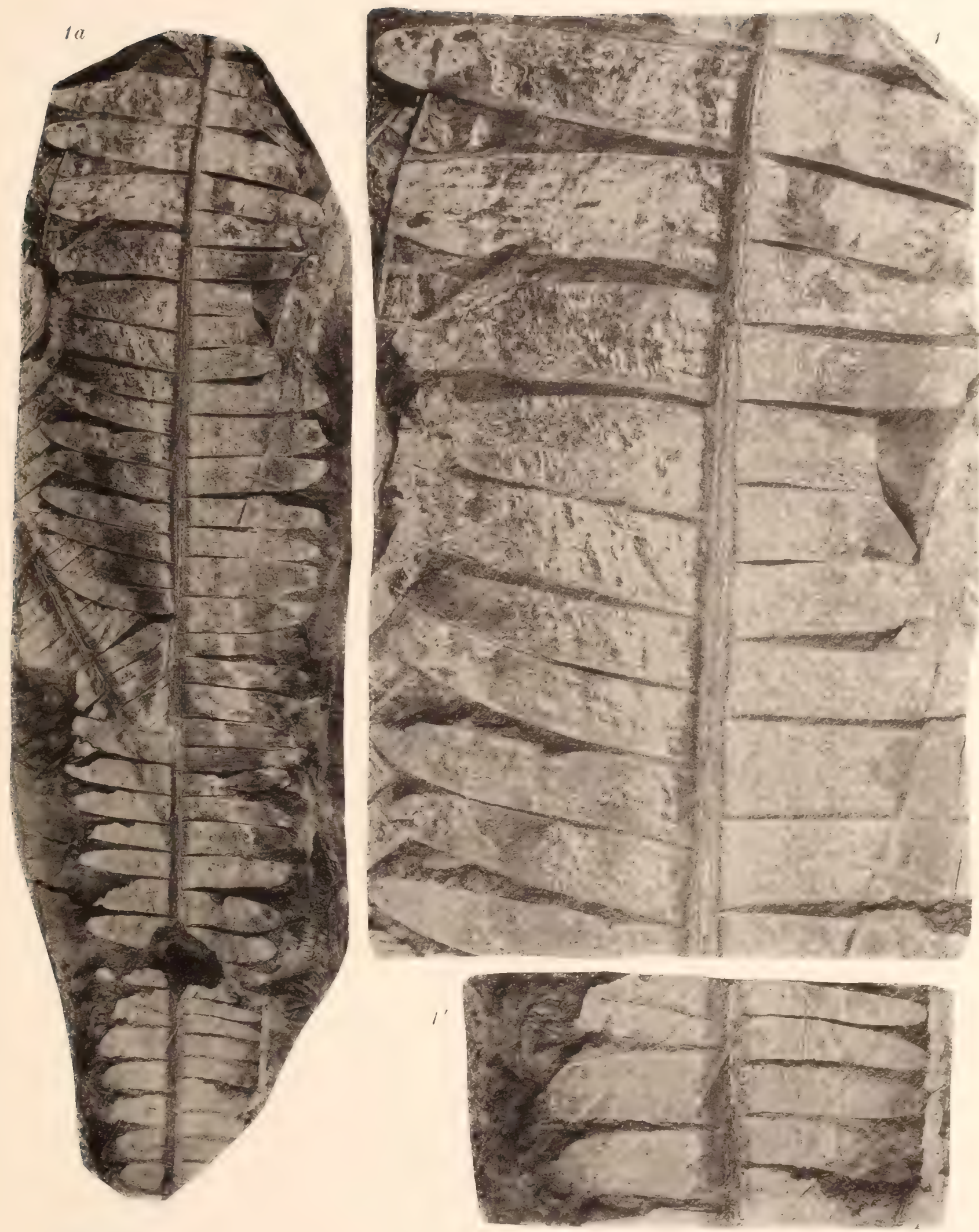

Clichés: sohice

Photuty]u situm

1.1.111111211) - 11:11111 -1.111 
. 
PLANGHE XLVIII

12. 


\section{PLANCHE XLVIII.}

\section{EXPLICATION DES FIGURES.}

Fig. 1. - Pterophyllum contiguum Schenk. - Sommet d'une fronde.

Hongaỹ, mine de Carrère, au toit de la couche Marmottan.

Fig. 2. - Pterophyllum contiguum Schenk. - Fronde incomplète.

Mines de Hongaỹ : Hatou, grande couche, grand banc de schistes.

Fig. 2a. - Portion du même échantillon, grossie deux fois.

Fig. 3. - Pterophyllum contiguum Schenk. - Fronde de petite taille, complète.

Mines de Hongaỹ : Hatou, grande couche, grand banc de schistes.

Fig. 4. - Pterophyllum contiguum Schenk. - Fragment de fronde.

Hongaỹ, mine de Carrère, au toit de la couche Marmottan.

Fig. 4a, 4 b. - Portions du même échantillon, grossies deux fois.

Fig. 5. - Pterophyllum contiguum Schenk. - Fragment de fronde.

Hongaÿ, au toit de la couche Marguerite.

FIG. 5 a. - Portion du même échantillon, grossie deux fois.

FIG. 6. - Pterophyllum contiguum Schenk. - Fragments de frondes.

Hongaỹ, mine de Carrère, au toit de la couche Marmottan.

Fig. 7. - Pterophyllum contiguum Schenk. - Portion supérieure d'une fronde.

Hongaÿ, rivière des Mines, mine Marguerite.

Fig. 8. - Pterophyllum contiguum Schenk. - Fragment de fronde.

Hongaý, au toit de la couche Marguerite. 

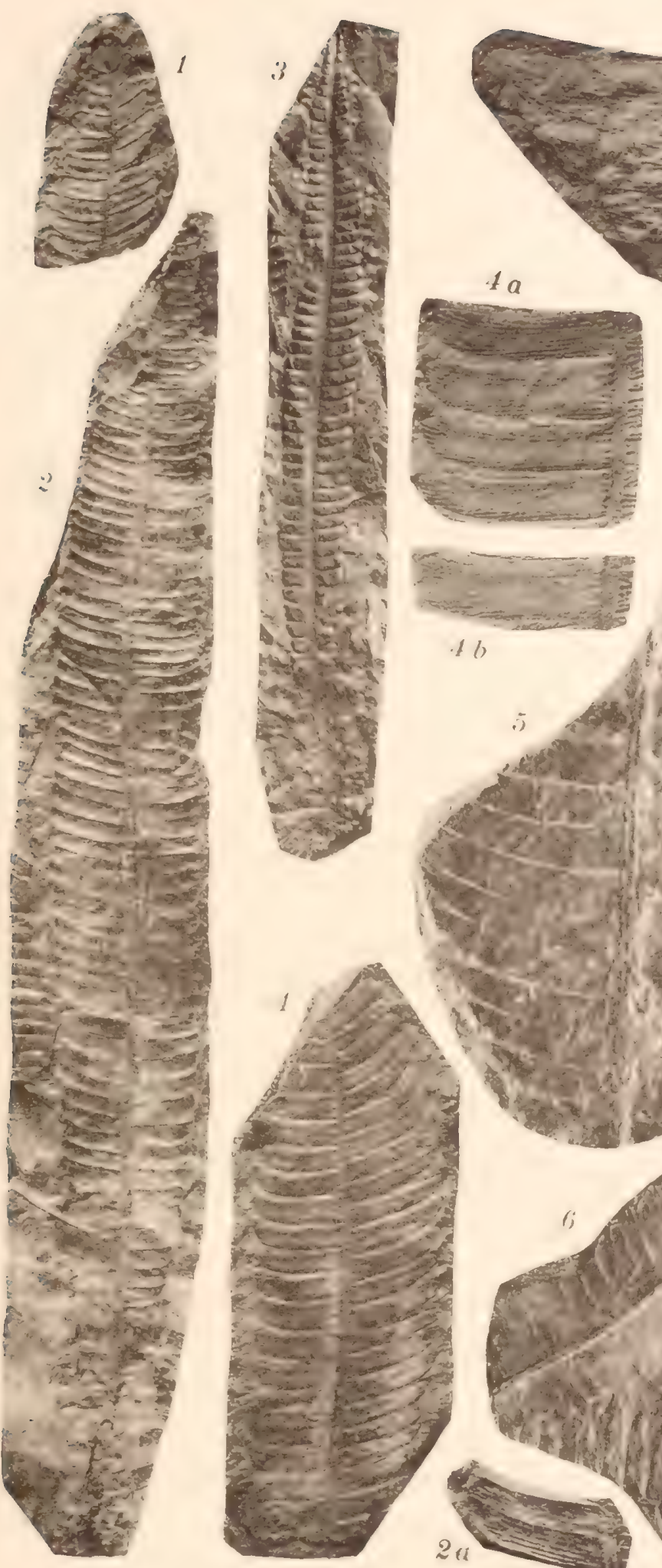

2) $2: ! 11$

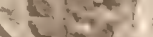

ace by

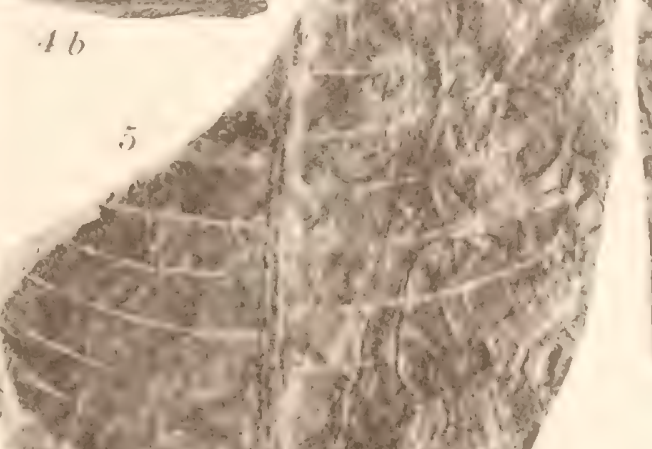

in

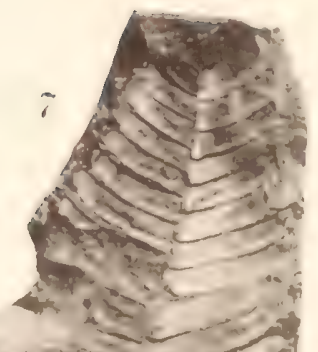

(1)

I. 420

iो $2 x$ का

b. $-2 x$

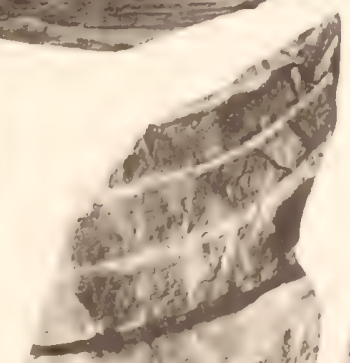

sing $x+1$

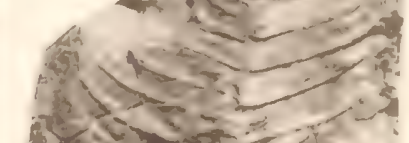

$12 x-5=2.5$

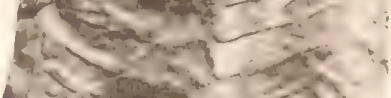

NI

destas $=$ $2 x^{x}+x^{2}$

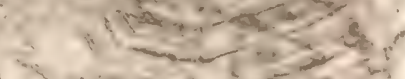
$x+2-13-2$ th

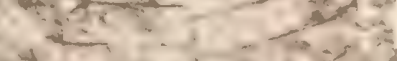
th $=1-10=2$ $-17 x=-12 \sin$ Thin-a.

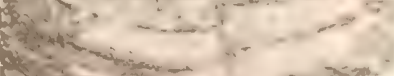

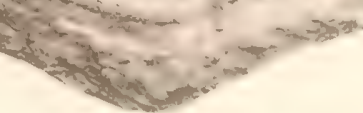
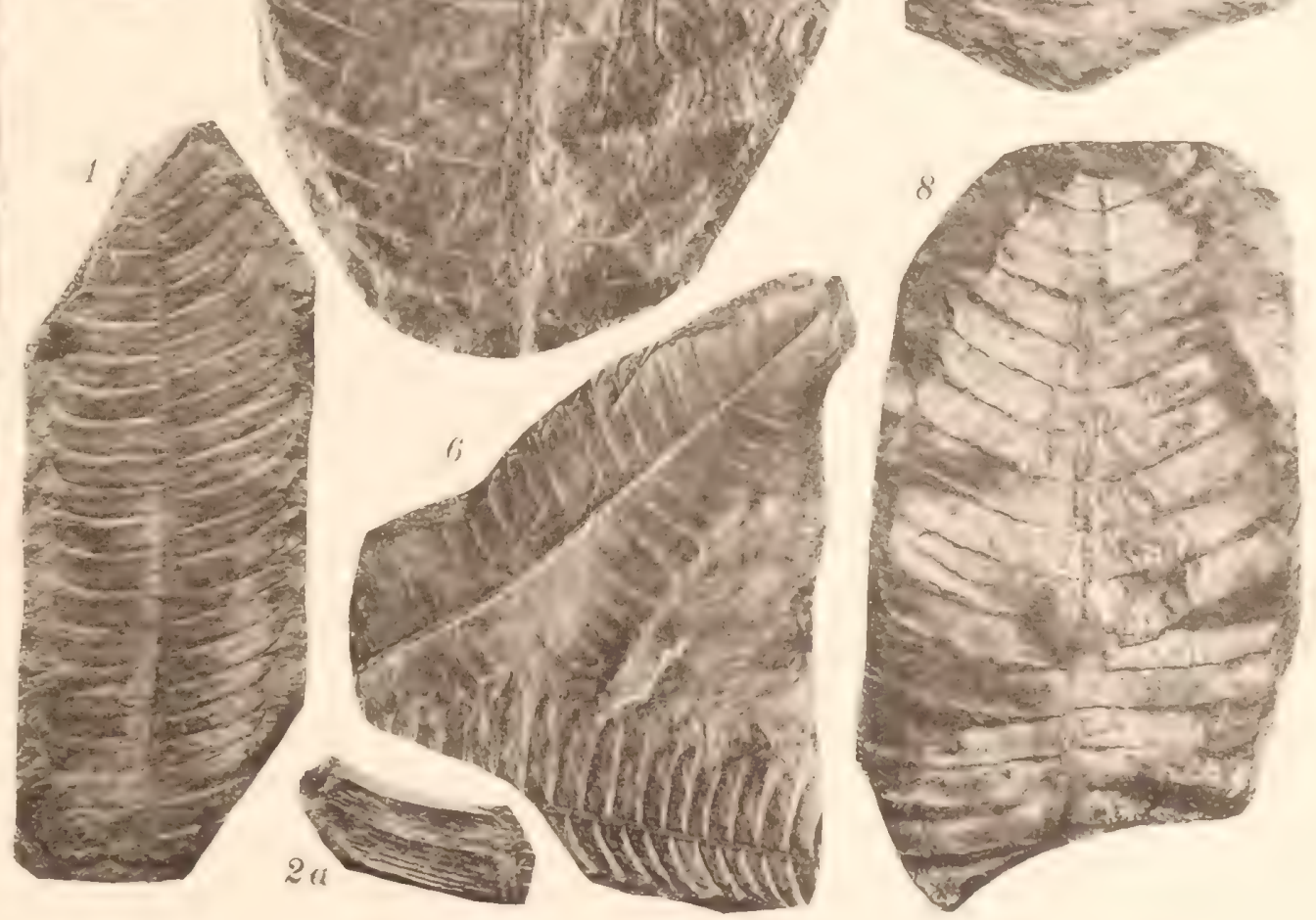

Clichés iohie 



\section{PLANCHE XLIX}




\title{
PLANCHE XLIX.
}

\author{
EXPLICATION DES FIGURES.
}

FIG. 1. - Pterophyllum Bavieri n. sp. - Fragment de fronde.

Mines de Hongaỹ, découvert de Hatou.

lig. 2. - Pterophyllum Bavieri n. sp. - Fronde presque complète.

Mines de Hongaỹ, découvert de Hatou.

FIs. 3. - Pterophyllum Bavieri n. sp. - Fragments de frondes.

Mines de Hongaÿ: Hatou, au toit de la grande couche.

Fig. 3 a. - Portion du même échantillon, grossie deux fois.

Fig. 4. - Pterophyllum æquale Brongniart (sp.). - Fronde presque complète.

Hongaÿ, vallée orientale de l'OEuf, galerie Léonice.

Fig. 5. - Pterophyllum æquale Brongniart (sp.). - Sommet d'une fronde.

Mines de Hongaÿ: Hatou, grande couche, grand banc de schistes.

finc. 6. - Pterophyllum æquale Brongniart (sp.). - Portion inférieure d'une fronde.

Mines de Hongaÿ: Hatou, grande couche, grand banc de schistes.

Fis. 6 i. - Portion du même échantillon, grossie deux fois.

Fig. 7. - Pterophyllum æquale Brongniart (sp.). - Fragment de frönde.

Mines de Hongaÿ: Hatou, grande couche. 
Pl. XLIX
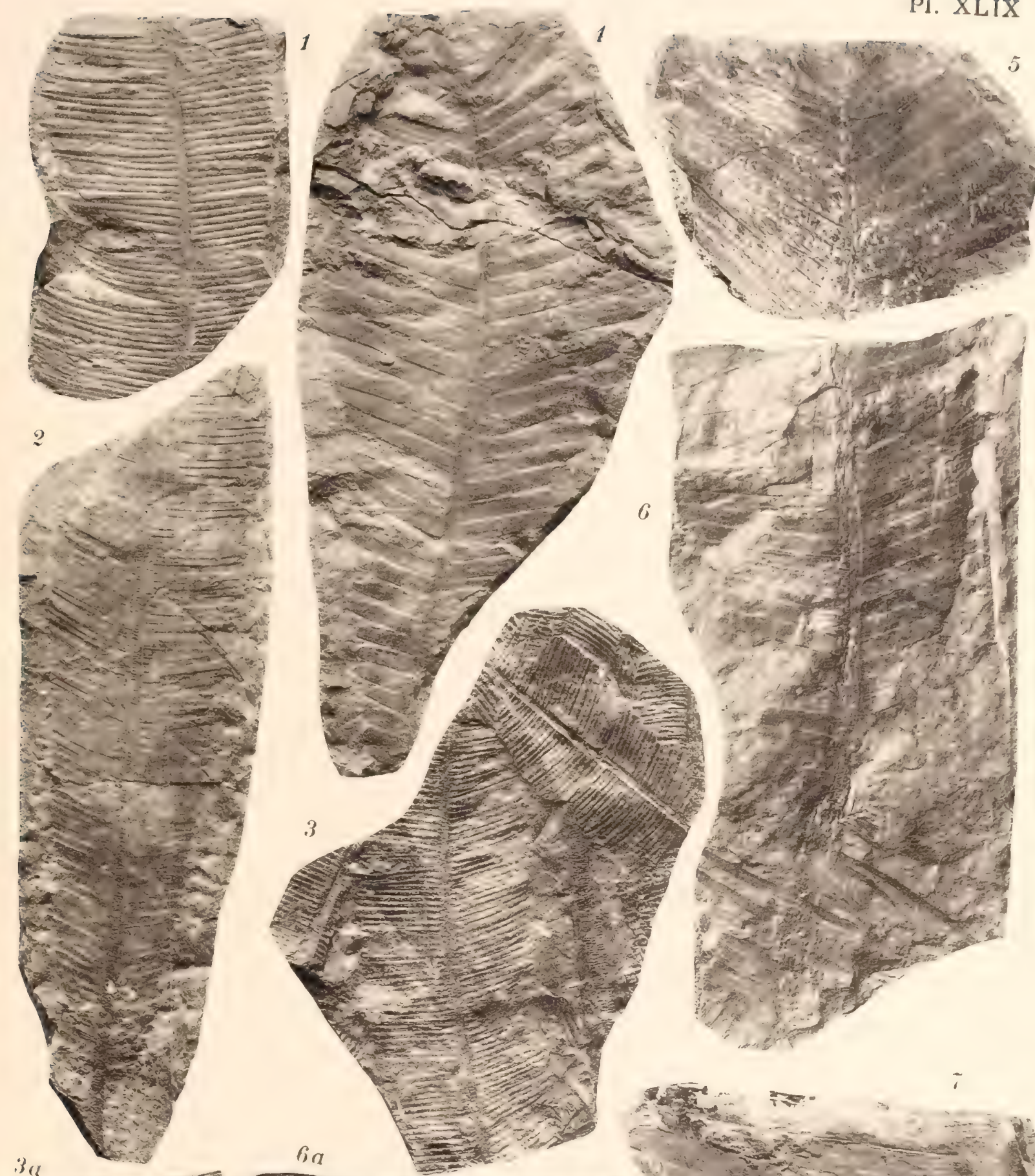

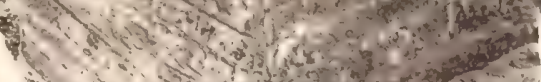
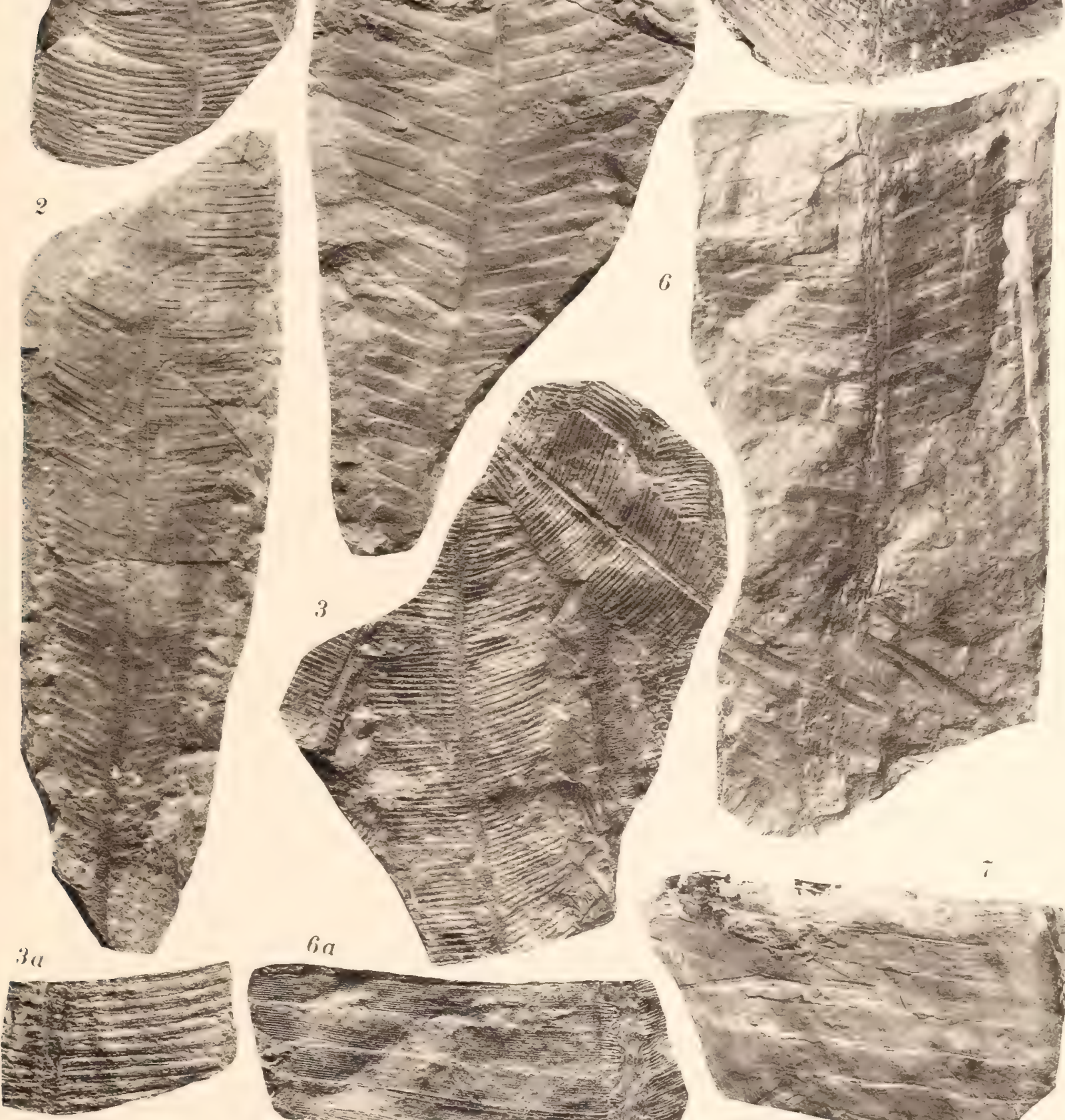

fin

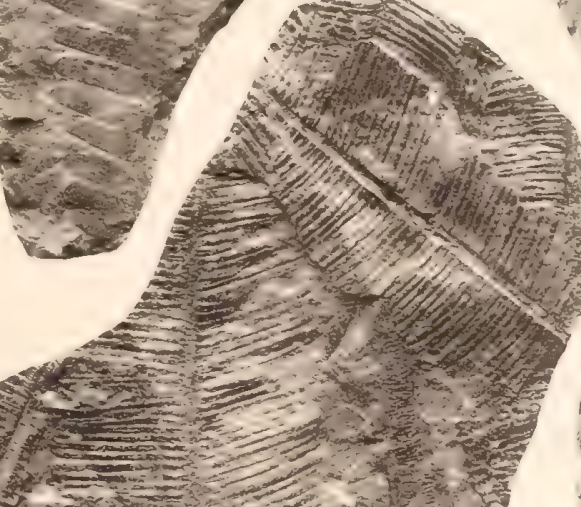

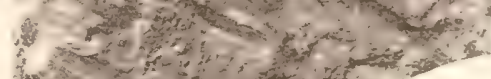

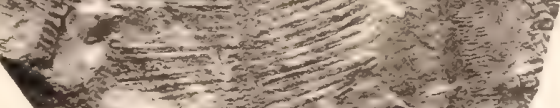

3. MAt

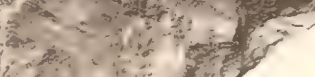

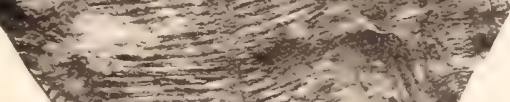

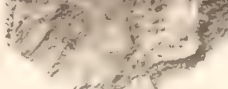
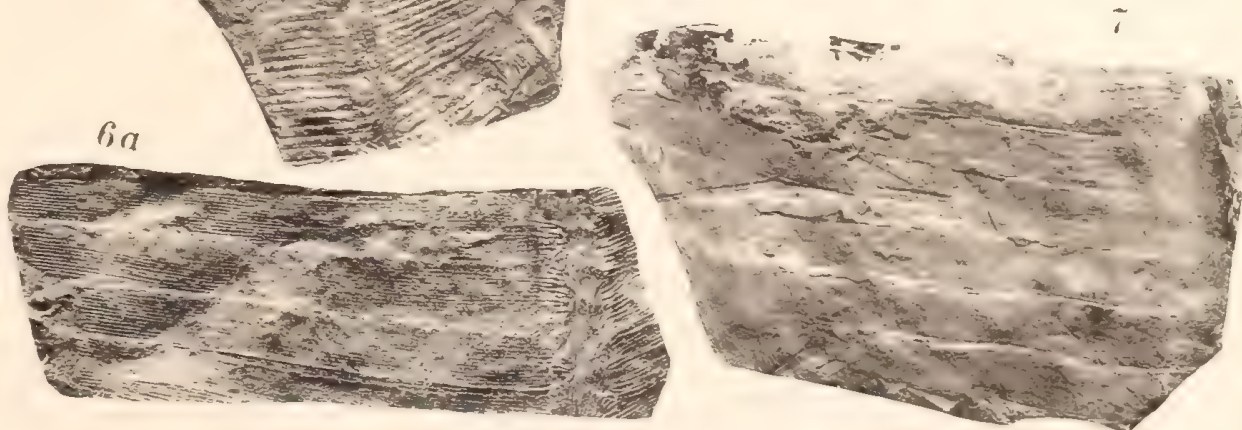

Clichẻs Sonier. 

PLANCHE L 


\section{PLANGHE L.}

\section{EXPLICATION DES FIGURES.}

FIG. 1. - Cycadolepis corrugata n. sp. - Écaille détachée de Cycadinée.

Hongaÿ, mine de Carrère, au toit de la couche Marmottan.

Fig. 2. - Cycadolepis corrugata n. sp. - Écaille dełachée de Cycadinee.

Hongaÿ, mine de Carrère, au mur de la couche Chater.

Fig. 3 et 4. - Cycadolepis corrugata n。 sp. - Écailles détachees de Cycadinées, plus ou moins incomplètes.

Hongaÿ, vallée orientale de lOEuf, galerie Léonice.

FIg. 4a. - Portion de l'échantilton fig. 4, grossie une fois et demie.

Frg. 5. - Cycadolepis granulata n. sp. - Écaille détachée de Cycadinée.

Kébao, mine Rémaury.

Fís. 6. - Cycadolepis cf. villosa SAPorta. - Écaille détachée de Cycadinée (figurée Bull. Soc. Gréol. de France, $3^{\circ}$ série, t. XIV, pt. XXV, fig. 4).

Bassin de Hongaÿ.

Fig. 7. - Échantillon d'attribution incertaine, pouvant faire songer à une base d'appareil fructificateur de Williansonia.

Mines de Hongaỹ : Hatou, au mur de la grande couche.

FIG. 8. - Écailles d'attribution incertaine.

Nines de Hongaÿ: Hatou, grande couche, grand banc de schistes.

FIG. 9. - Conites sp. - Còne de fructification d'attribution incertaine, fendu longitudinalement :

Hongaÿ, île du Sommet Buisson, tranchée en avant de la galerie Jean.

Fig. 9a. - Portion du même échantillon, grossie deux fois.

FIG. 10. - Conites sp. - Còne de fructification d'attribution incertaine, vu extérieurement.

Mines de Hongaỹ : Hatou, grande couche, grand banc de schistes.

Fig. 11. - Conites sp. - Cỏne de fructification d'attribution incertaine, vu extérieurement. Hongaÿ, rivière des Mines, mine Marguerite.

FIG. 12. - Conites sp. - Cône de fructification d'attribution incertaine, vu extérieurement. Mines de Hongay : Hatou, grande couche, grand banc de schistes.

FIG. 13 et I4. - Gonites sp. - Débris de cône et cône de fructification à écailles hexago nales rappelant le Kaidacarpum sibiricum Heer.

Mines de Hongaÿ: Hatou, au mur de la grande couche.

FIG. 15. - Conites Leclerei n. sp. - Empreinte d'un cône de fructification d'attribution incertaine.

Kébao.

Fig. 15a. - Portion du même échantillon, grossie deux fois.

FIG. 16. - Baiera Guilhaumati n. sp. - Feuille complète.

Mines de Hongaÿ : Nagotna, au mur de li couche Bavier.

Fig. 17 et 18. - Baiera Guilhaumati n. sp. - Portions de feuilles.

Mines de Hongaỹ : Nagotna, au mur de la couche Bavier.

Fig. 17a. - Portion de feuille de l'échantillon lig. 17, grossie une fois et demie.

FIG. 19. - Baiera Guilhaumati n. sp. - Fragments de feuilles.

Mines de Hongaÿ: Nagotna.

FIG. 20. - Organes d'attribution problématique (figurés Bull. Soc. Géol. de France, $3^{\circ}$ série, t. XIV, pl. XXV, fig. 5).

Bassin de Hongaÿ.

FIG. 20a. - Portion du mème échantillon, grossie une fois et demie. 
PI. L
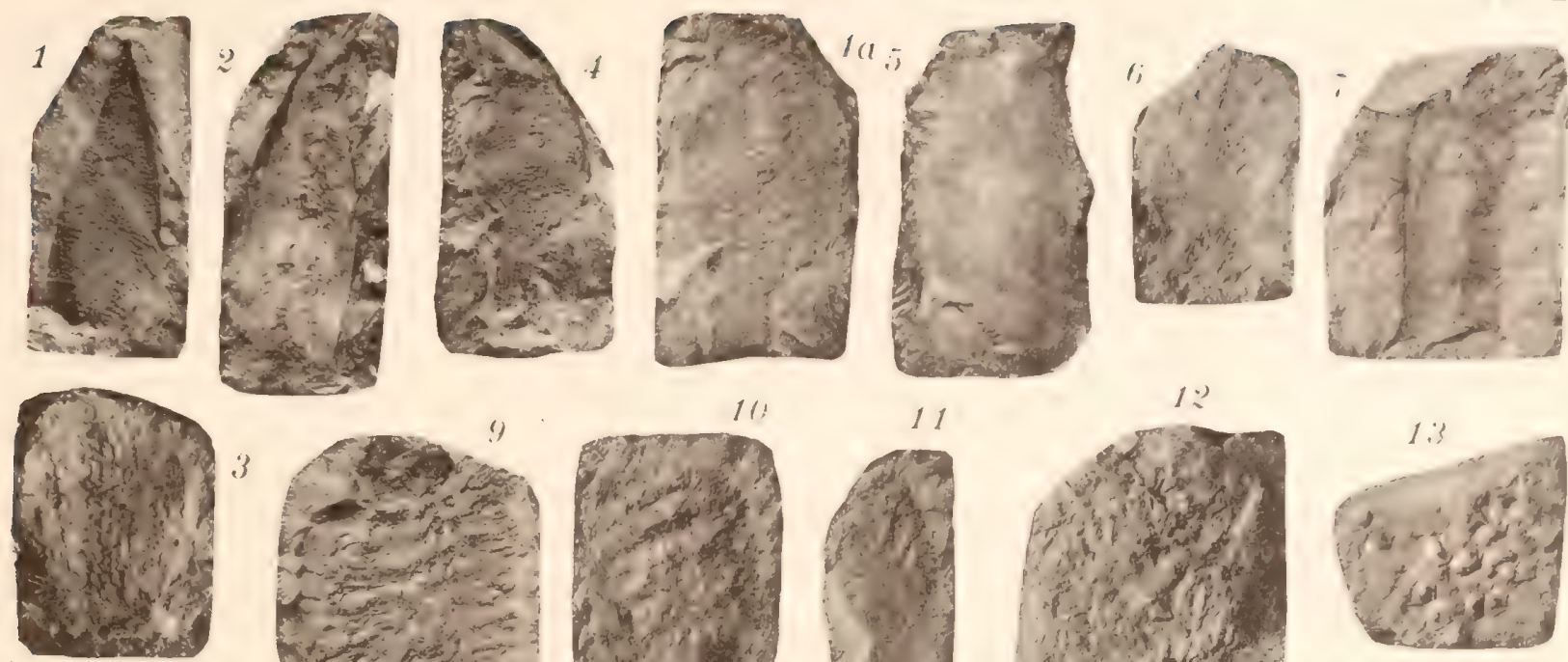

11:
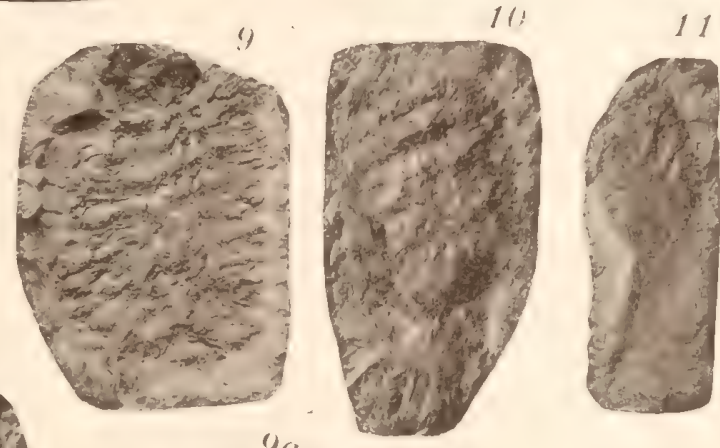

11

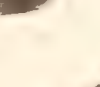

1:
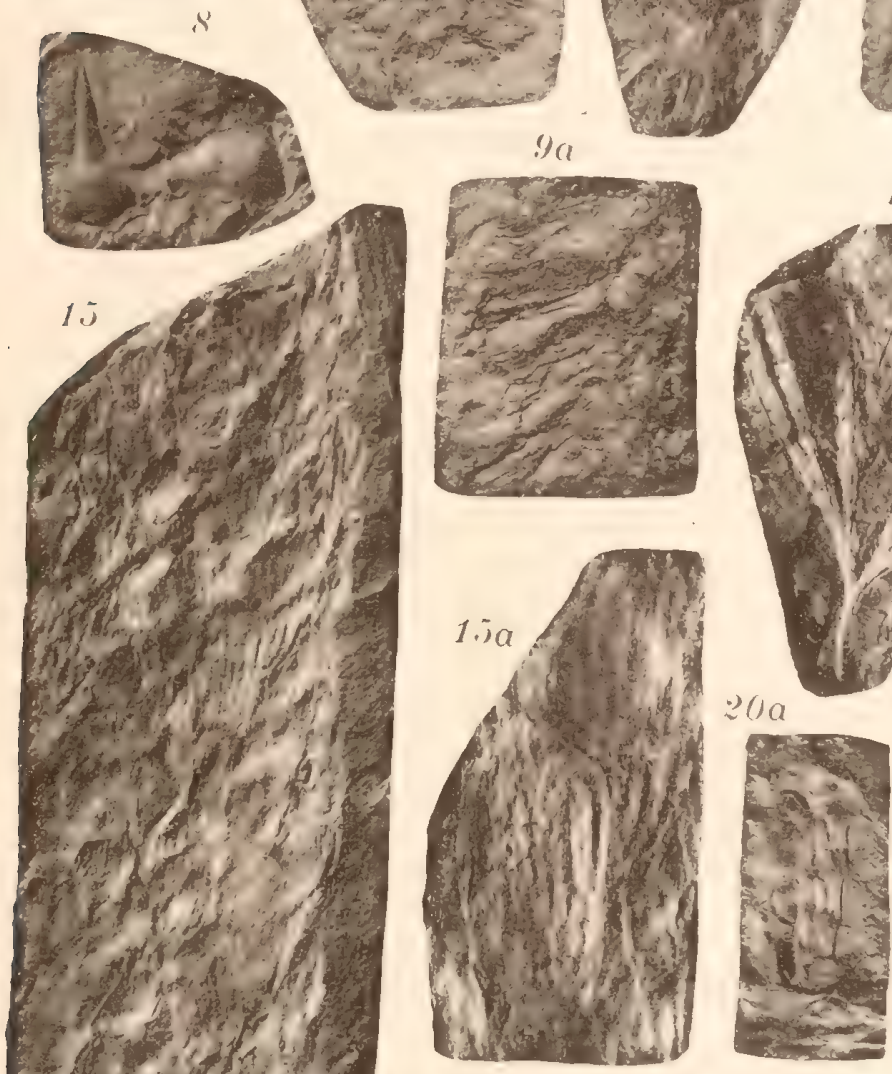

I i
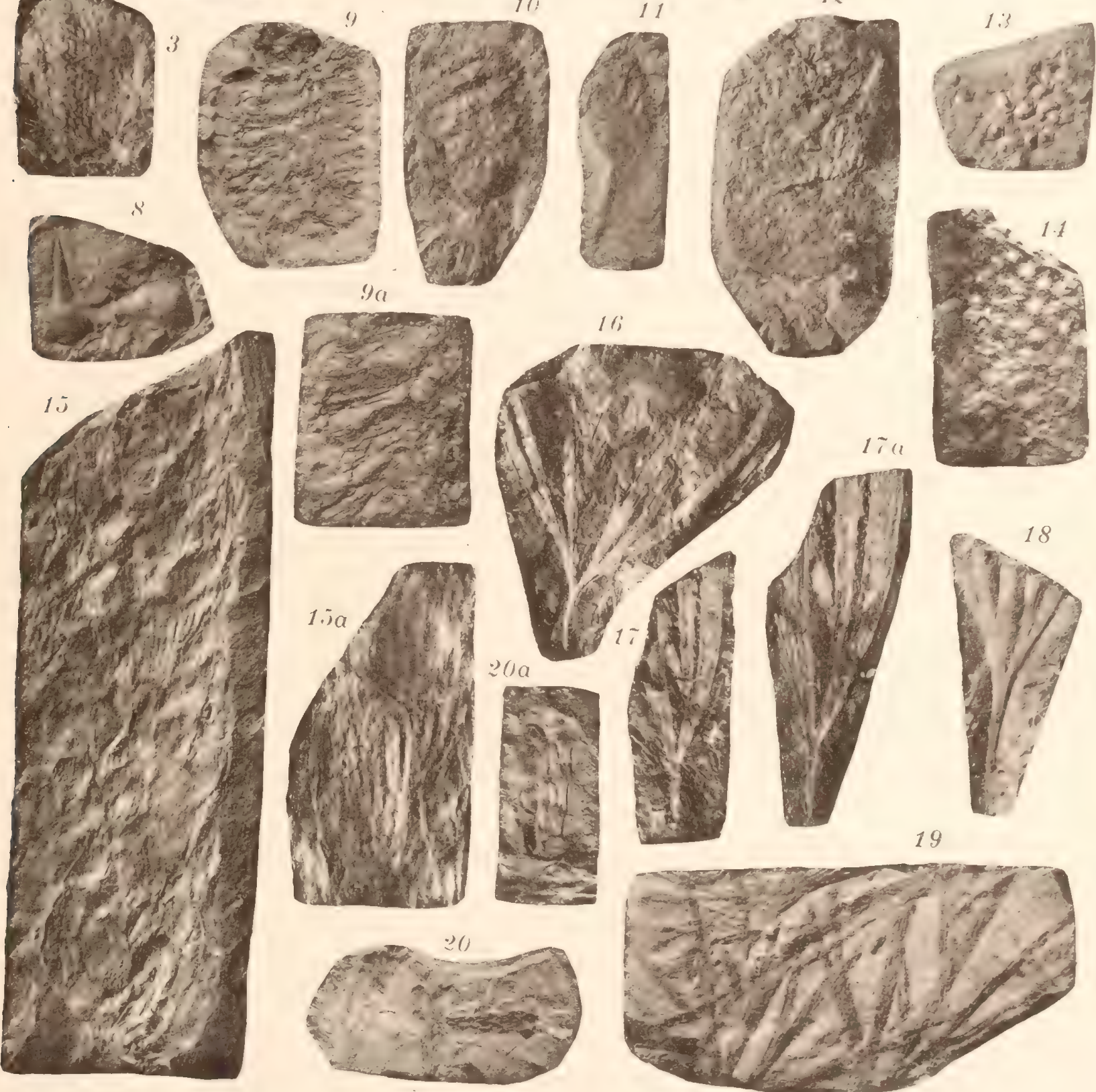

Clichés Sohier.

Photorypie Sohier - Champigny-s/Marne (Seine)

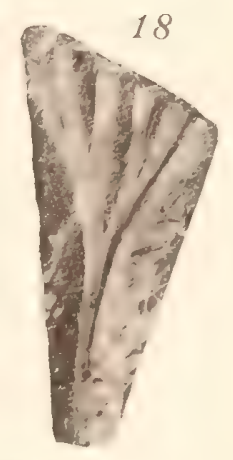

19 



\section{PLANCHE LI}




\section{PLANGHE LI.}

\section{EXPLICATION DES FIGURES.}

FIG. 1 (à gauche). - Angiopteris (?) sp. - Fragment de penne.

Yen-Baï,

FIG. 1 (à droite). - Selliguea sp. - Fragment de fronde.

Yen-Bai.

FIG. 1a. - Portion de la même penne, grossie deux fois.

Fig. 2. - Salvinia formosa HeEr. - A la partie supérieure de l'échantillon, deux feuilles encore groupées par paire; vers le bas, une seule des feuilles de la paire voisine.

Yen-Baï.

FIG. 2a. - Feuille du même échantillon, grossie deux fois.

Fig. 2a'.- Portion de la même feuille, grossie quafre fois.

FIG. 3. - Salvinia formosa HeEr. - Feuille isolée.

Yen-Baï.

Fır. 4 à 13. - Ficus Beauveriei n. sp. - Fragments de feuilles et feuilles plus ou moins complètes, montrant les variations de forme et de taille.

Yen-Bai.

FIG. 8a. - Portion de l'échantillon fig. 8, grossie cinq fois. 
1) I,
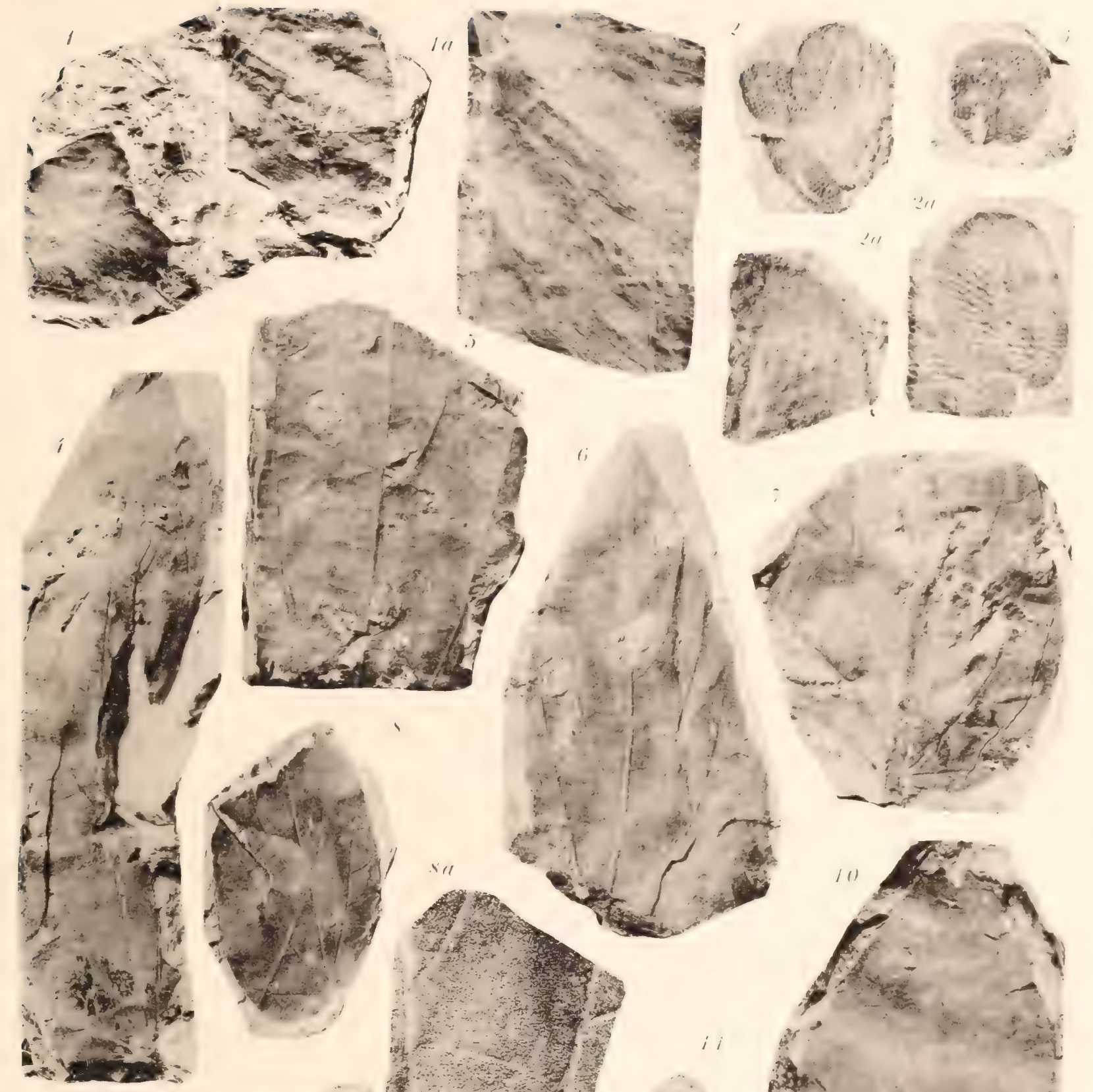

ti
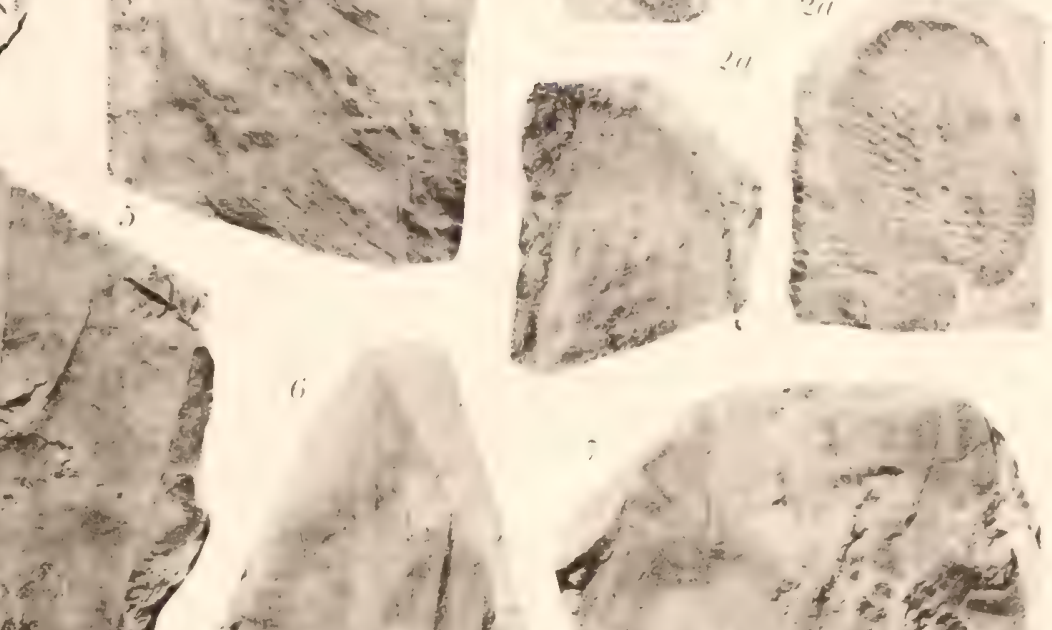

sid

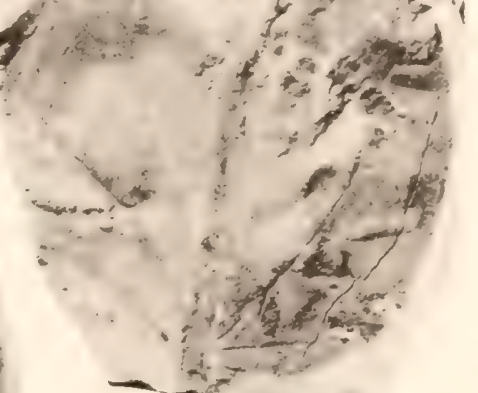

to

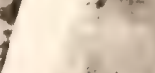




$$
\text { . }
$$


PLANCHE LII

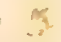

13. 


\section{PLANGHE LII.}

\section{EXPLICATION DES FIGURES.}

Fig. 1. - Flabellaria sp. - Base d'une feuille flabellée de Palmier. Yen-Baï.

Fig. 2. - Poacites sp. - Fragment de feuille rubanée appartenant à une Monocotylédone.

Yen-Baī.

Fig. 2a. - Portion du même fragment de feuille, grossie deux fois.

FIG. 3. - Phyllites sp. - Fragment d'une grande feuille de Dicotylédone, d'attribution incertaine.

Yen-Baï.

FIG. 4. - Phyllites sp. - Feuille de Dicotylédone incomplète, d'attribution incertaine. Yen-Baï.

FIG. 5. - Phyllites sp. - Feuille de Dicotylédone incomplète, d'attribution incertaine. Yen-Baï.

FIG. 6. - Phyllites sp. - Feuille de Dicotylédone incomplète, d'attribution incertaine. Yen-Baï.

FIG. 7. -. Fruit ou graine, d'attribution incertaine.

Yen-Baĩ. 


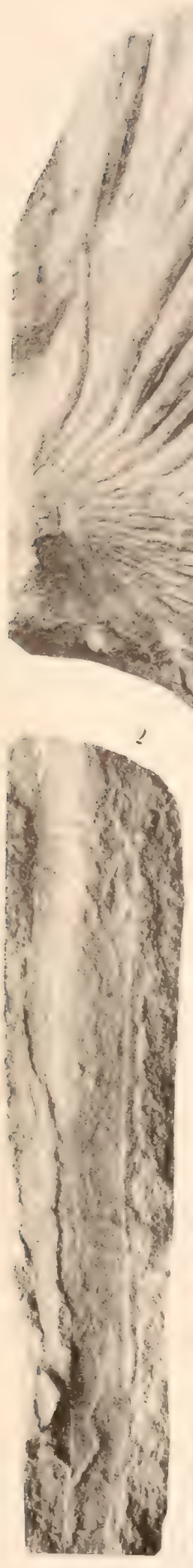

Clichés Sohie
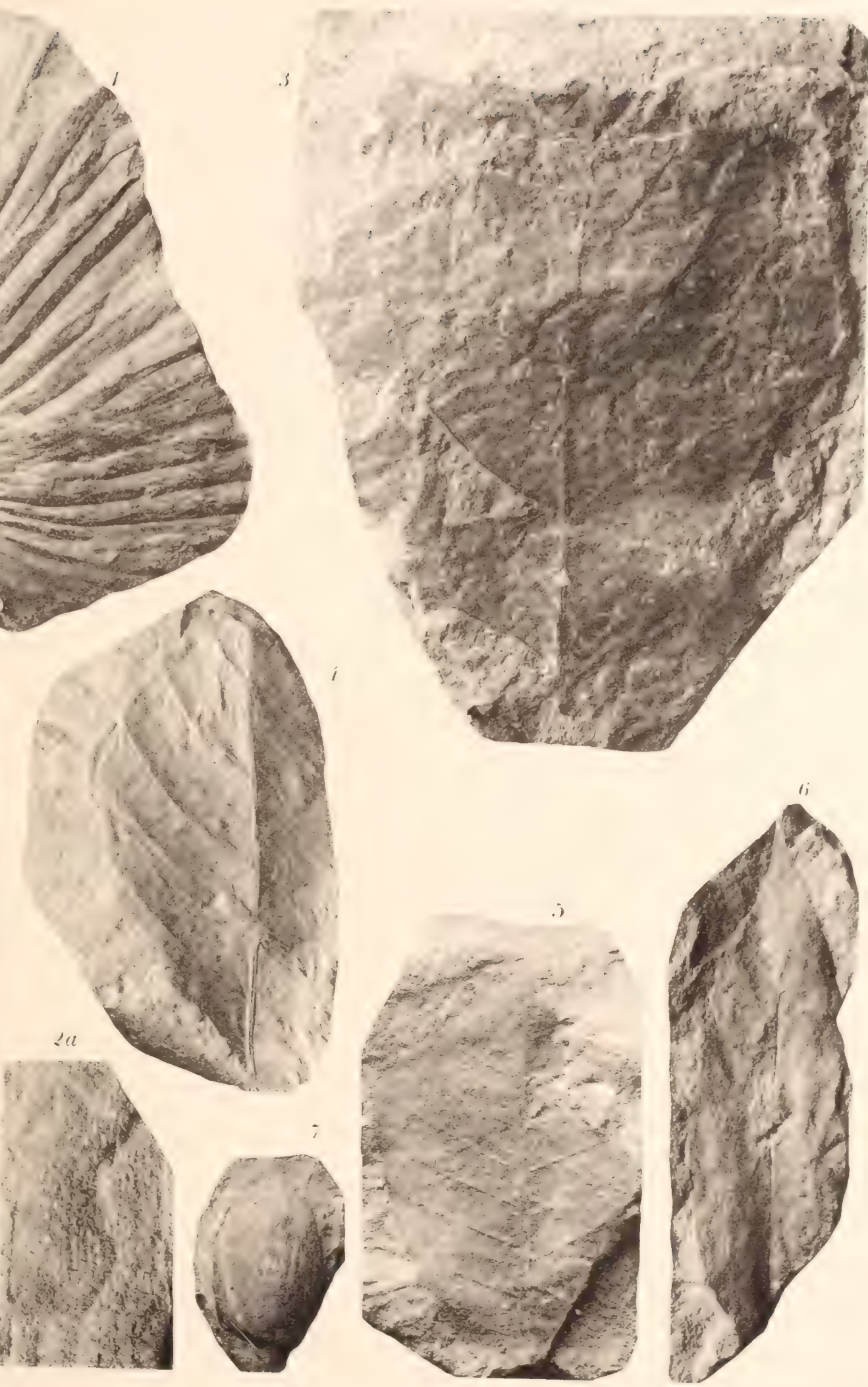

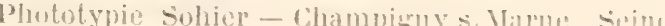





\section{PLANCHE LIII}




\section{PLANCHE LIII.}

\section{EXPLICATION DES FIGURES.}

Fig. 1. - Gerablattina elegans Scudder (n. sp.). - Aile détachée.

Mines de Hongaỹ : Hatou, grande couche.

FIG. Ia. — La même aile, grossie cinq̣ fois.

Fig. 2. - Etoblattina obscura Scudder (n. sp.). - Aile détachée, incomplète.

Kébao, système supérieur, couche $\mathrm{n}^{\circ}$ 2, galerie $\mathbf{M}$.

FIG. 2a. - La même aile, grossie cinq fois.

Fig. 3: - Etoblattina brevis Scudder (n. sp.). - Aile détachée.

Mines de Hongaÿ: Hatou, au mur de la grande couche.

Fig. 3 a. - La même aile, grossie cinq fois.

FIG. 4. - Coquille d'Ammonitidée (?). Moule charbonneux.

Mine de Trang-Back, galerie d'allongement Onest, au toit d'une couche.

Fig. 5. - Vivipara (Tylotoma) cf. Sturi Neumayr. - Coquille dégagée de la roche.

Yen-Baï; calcaires cristallins.

Fig. 5'. - Le même échantillon, vu de l'autre côté.

Fig. 6 à 9. - Vivipara (Tylotoma) cf. Sturi Neumayr. - Coquilles dégagées de la roche.

Yen-Baï; calcaires cristallins.

FIG. 10 et 11. - Unio sp. - Moules internes.

Yen-Baï; grès argileux.

N. B. - Les fig. I à 3 ne sont pas toutes trois arientées de même, l'orientation ayant été déterminée par le choix du meilleur éclairement. 

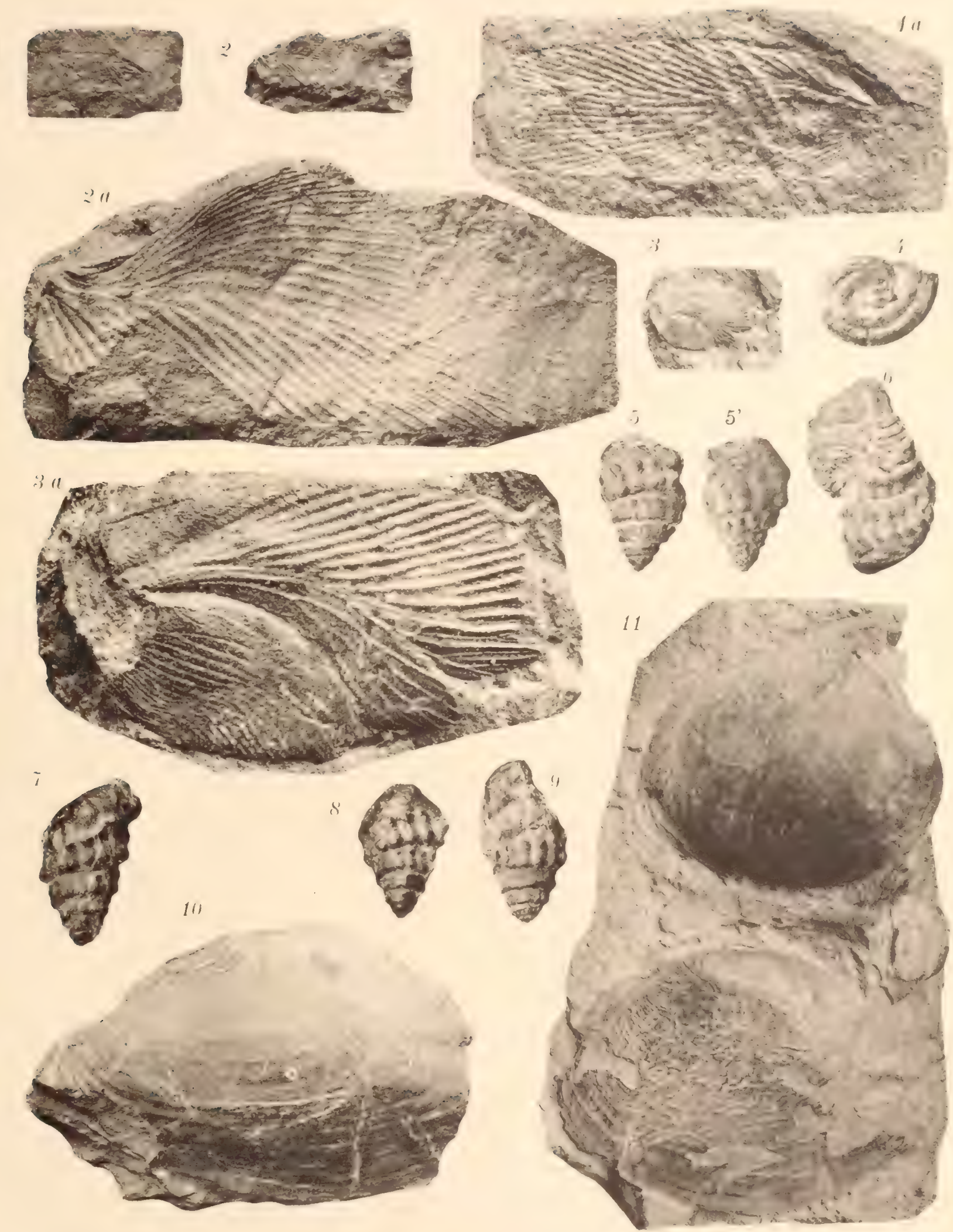

Clichés Solier

Phototypie Sohier - Champigny•s/Marne (Seine) 

PLANCHE LIV 


\section{PLANCHE LIV.}

\section{EXPLICATION DES FIGURES.}

Fig. 1 et 2. - Gladophlebis (Todea) Rosserti Presi (sp.). - Fragments de pennes. Taì-Pin-Tchang (Yunnan).

Fig. 3 et 4. - Gtenopteris Sarrani n. sp. - Fragments de frondes. Taï-Pin-Tchang.

Fig. 5. - Tæniopteris cf. immersa Nathorst. - Fronde incomplète. Tai-Pin-Tchang.

Fig. 5 a. - Portion du même échantillon, grossie une fois et demie. 
H'I. I.IY
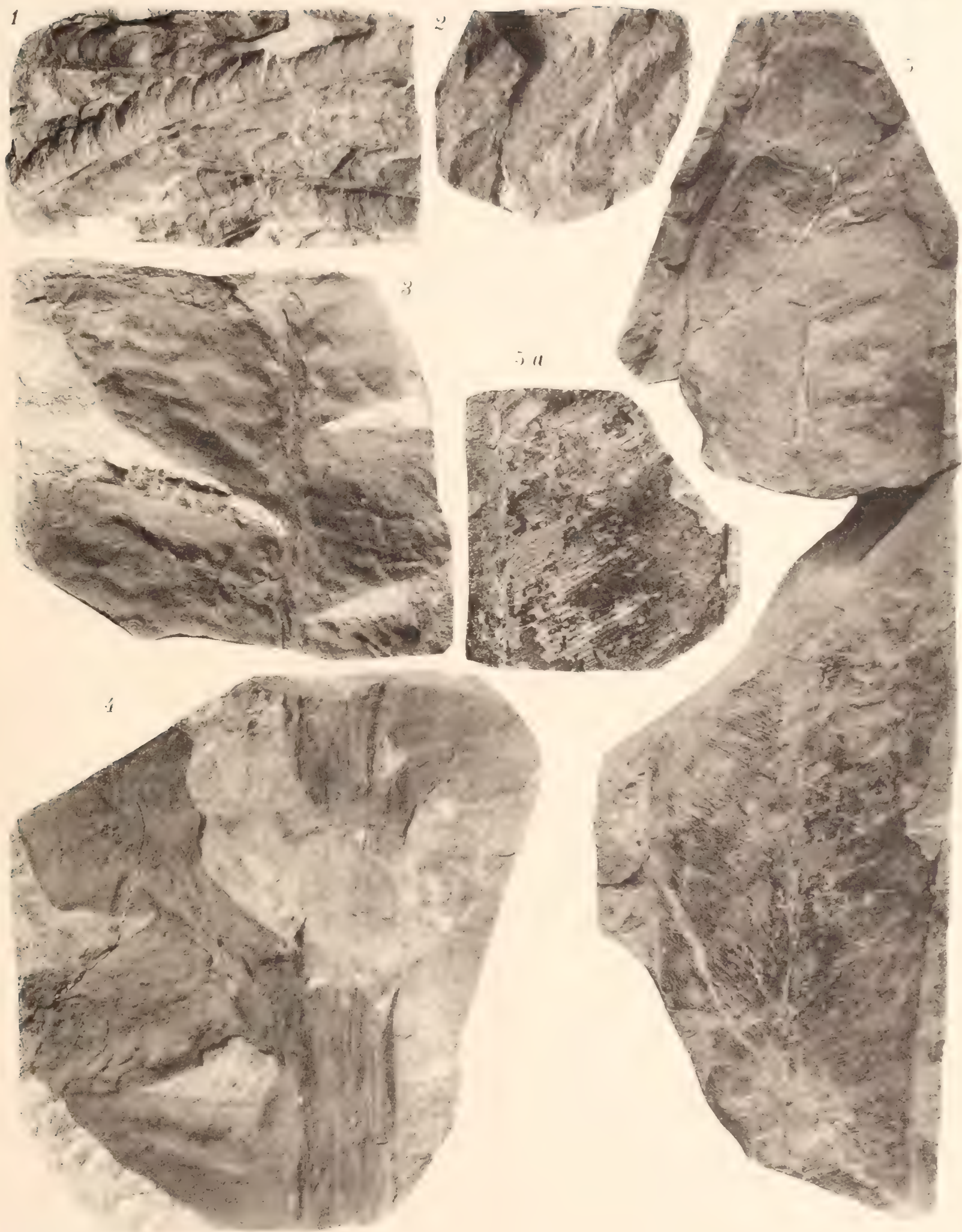



\section{PLANCHE LV}




\section{PLANCHE LV.}

\section{EXPLICATION DES FIGURES}

Fig. 1. - Tæniopteris Leclerei n. sp. - Fragment de fronde, vu en dessus. Tai-Pin-Tchang (Yunnan).

Fig. 1 a. - Portion du même échantillon, grossie deux fois.

Fig. 2. - Tæniopteris Leclerei n. sp. - Fragment de fronde, vu en dessous. Taï-Pin-Tchang.

FIG. 3. - Tæniopteris Leclerei n. sp. - Fragment de fronde, vu en dessus. Taï-Pin-Tchang.

FIG. 3 a. - Portion du même échantillon, grossie deux fois.

FIG. 4. - Tæniopteris Leclerei n. sp. - Frondes incomplètes, mais dont l'une, celle de droite, se suit jusqu'à son pétiole.

Taï-Pin-Tchang.

Fig. 4a. - Portion de l'une des frondes du même échantillon, prise vers l'angle supérieur de gauche, grossie deux fois. 
I'I I.I

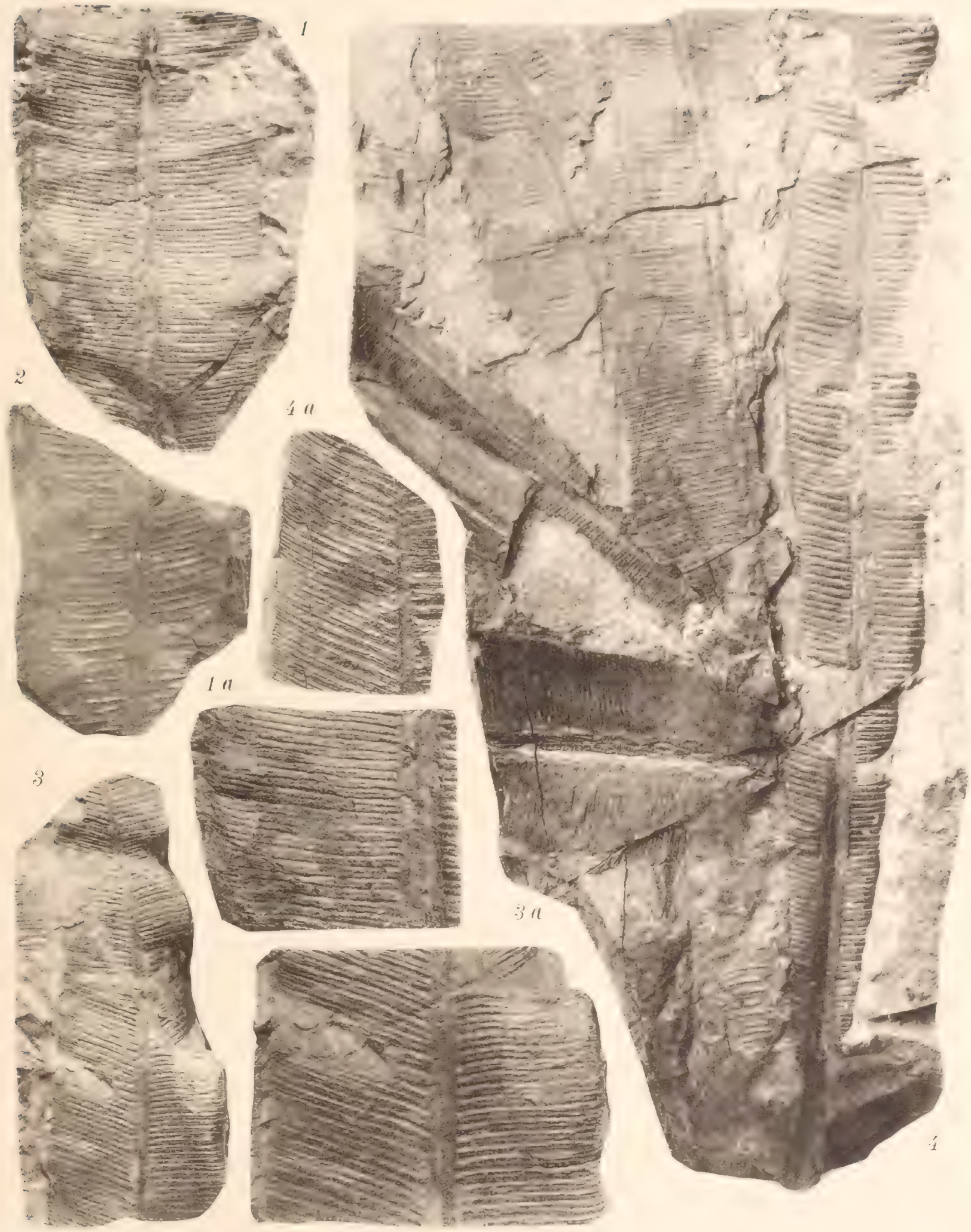

Cliches Sulnu 



\section{PLANCHE LVI}




\section{PLANCHE LVI.}

\section{EXPLICATION DES FIGURES.}

Fig. 1. - Glossopteris indica Schimper. - Fragment de fronde.

Taï-Pin-Tchang (Yunnan).

Fig. 1a. - Portion du même échantillon, grossie deux fois.

Fig. 2. - Glossopteris angustifolia Brongniart. - Partie supérieure d'une fronde.

Taï-Pin-Tchang.

Fig. 2a. - Portion du même échantillon, grossie deux fois et demie.

Fig. 3. - Dictyophyllum Nathorsti n. sp. - Fragment de penne.

Tai-Pin-Tchang.

FiG. 4. - Clathropteris platyphylla Gofppert (sp.). - Fragment de penne.

Taï-Pin-Tchang.

Fig. 5. - Pterophyllum sp. - Portion de fronde.

Taï-Pin-Tchang.

FIG. 6. - Pterophyllum (Anomozamites) inconstans Braun (sp.). - Fronde de petite taille, incomplète.

Taï-Pin-Tchang.

Fig. 7. - Ptilophyllum acutifolium Morris. - Fragment de fronde.

Taï-Pin-Tchang.

FIG. 7a. - Portion du même échantillon, grossie deux fois et demie.

Fig. 8. - Ptilophyllum acutifolium Morris, var. tenerrimam Feistuantel. - Fragment de fronde.

Taï-Pin-Tchang. 

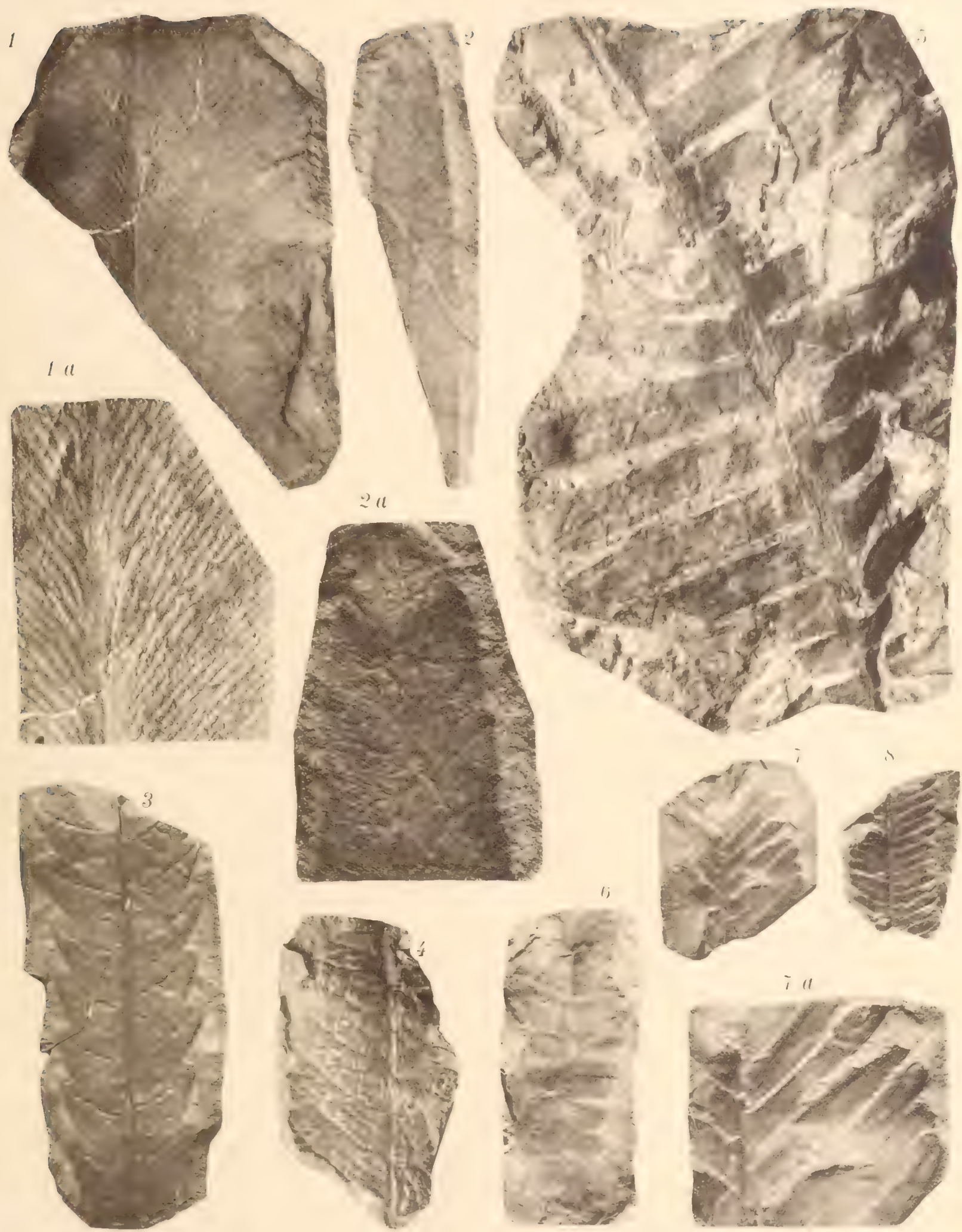

Clichés Solie:

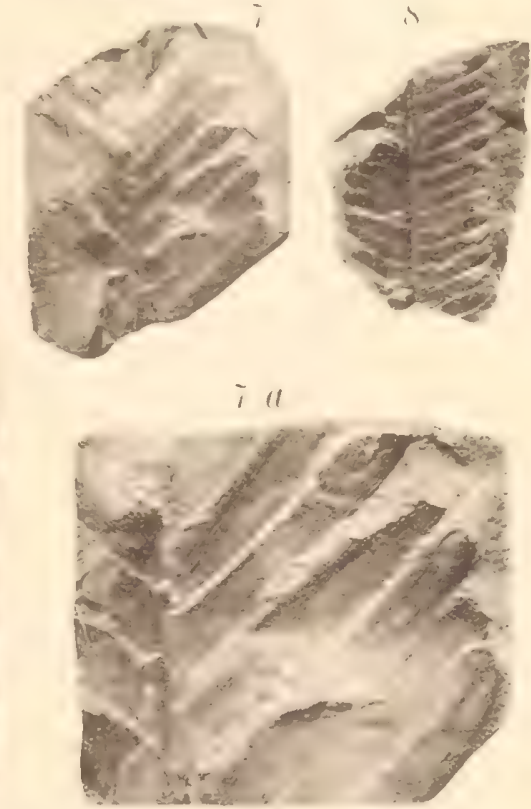

Phototypie Sohier - Champigny-s/Narne (Seine) 



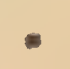








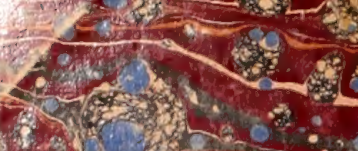

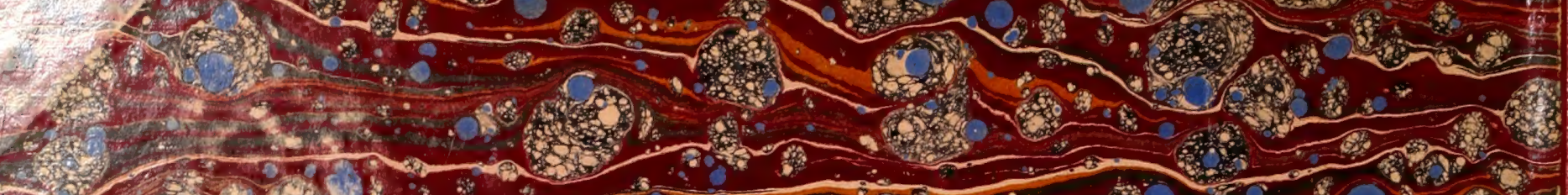
2.5.

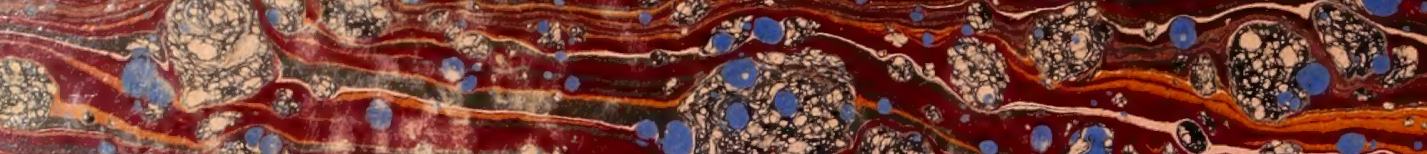

(5)

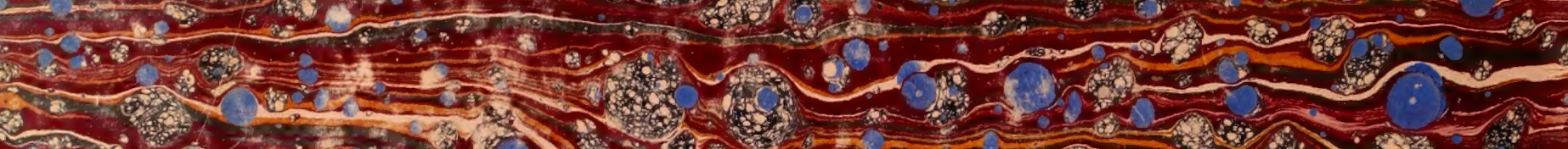

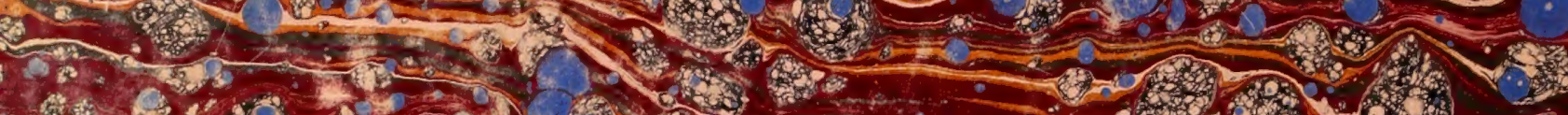

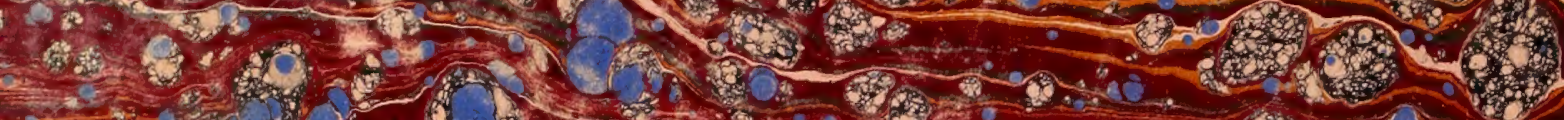

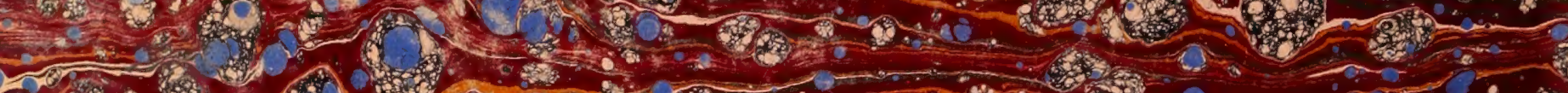

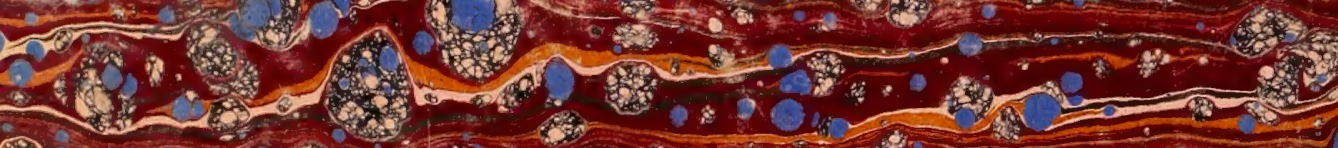

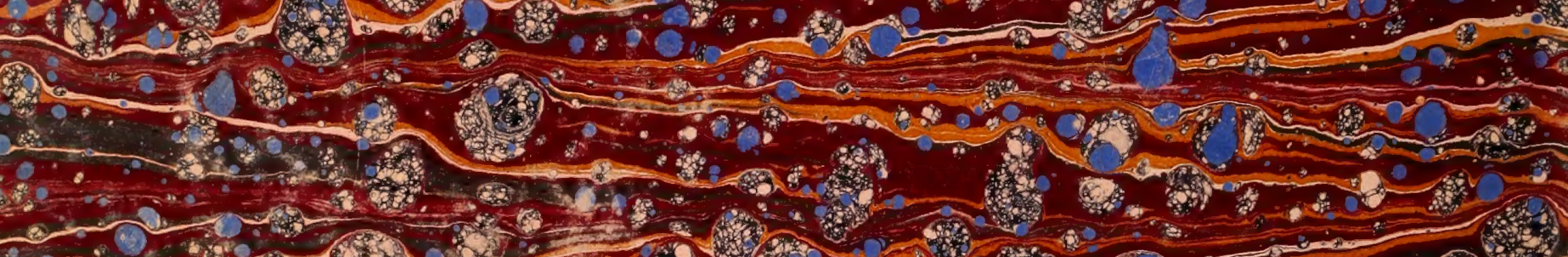

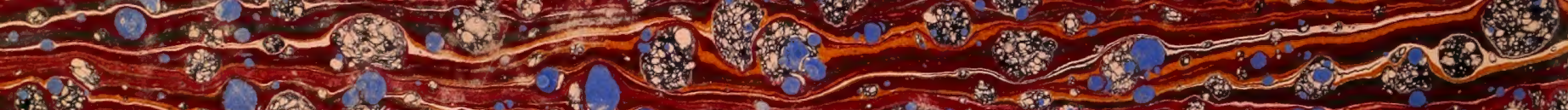

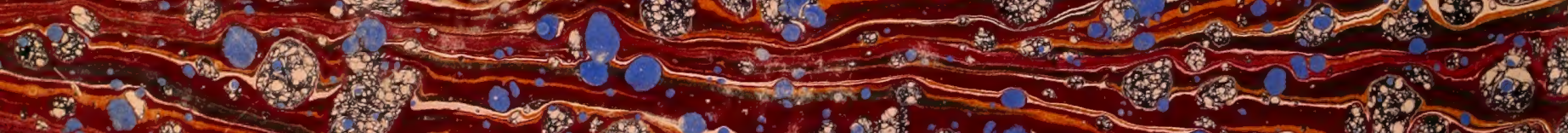

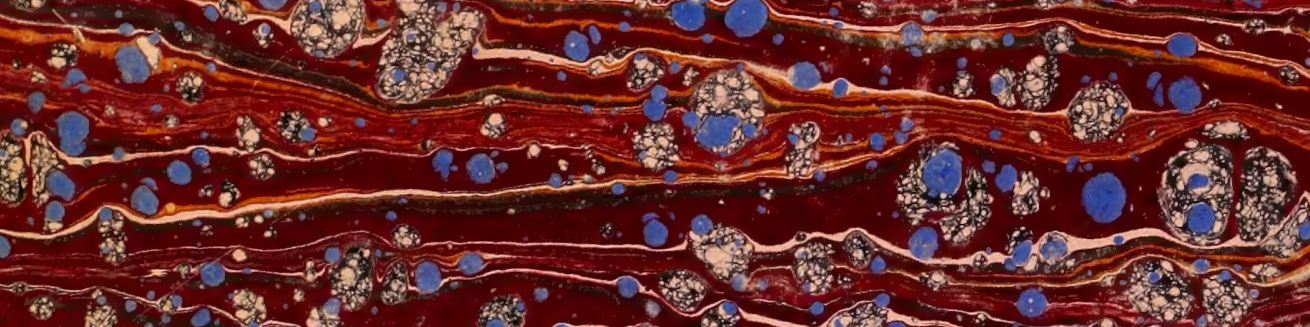

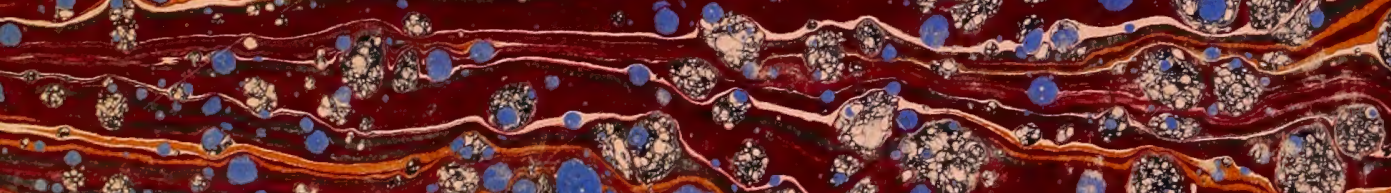

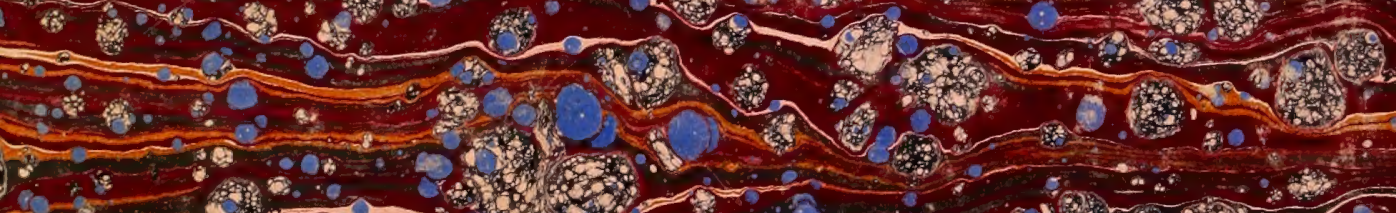

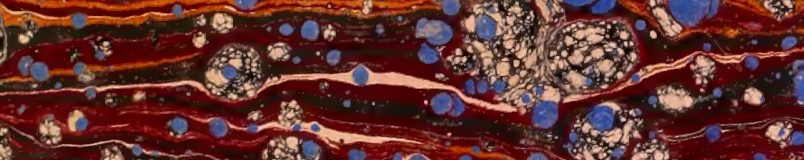

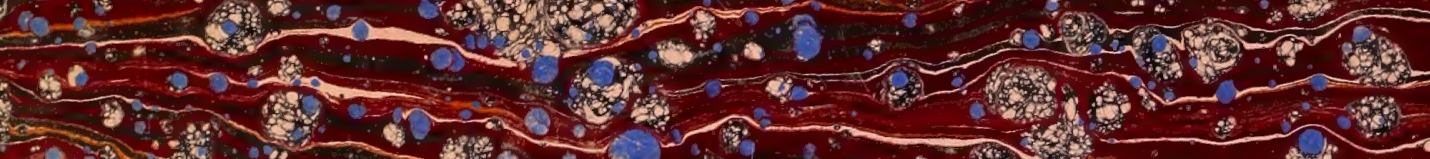

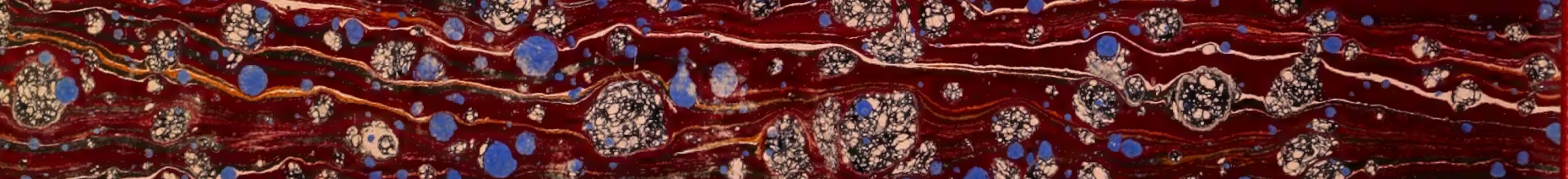

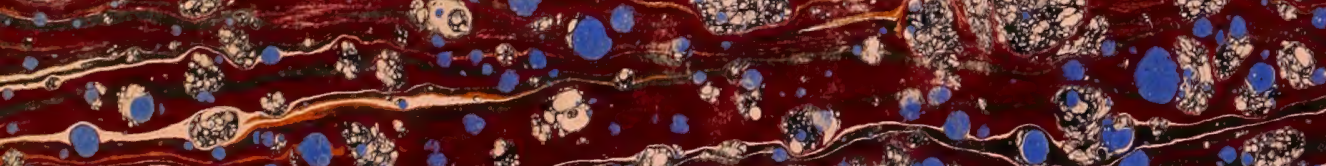

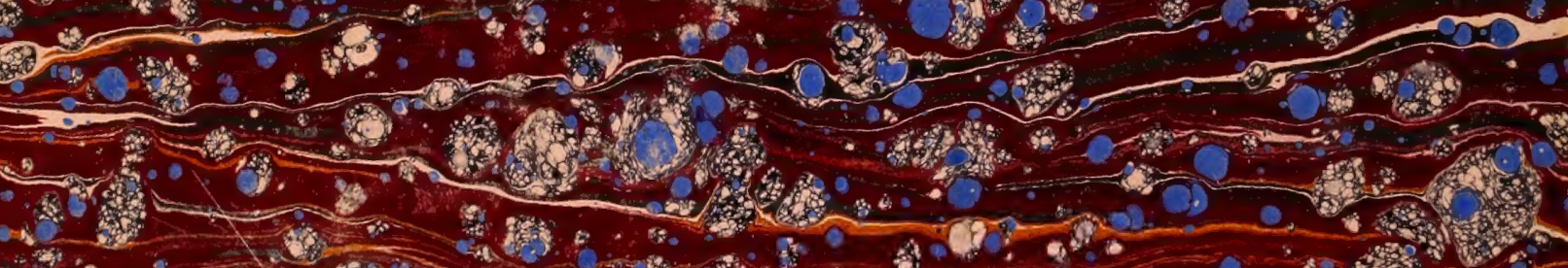

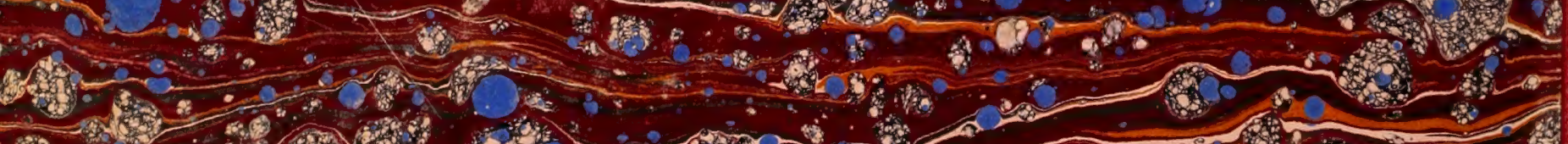

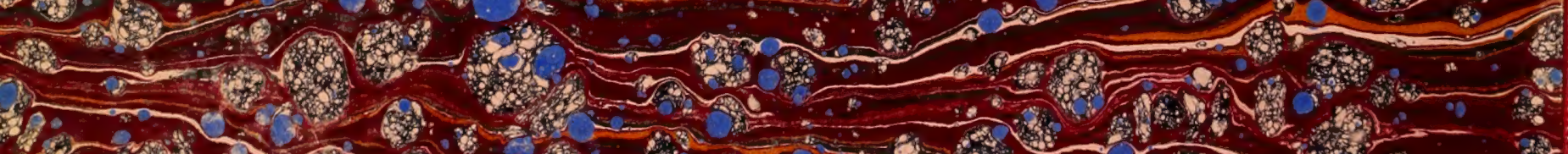

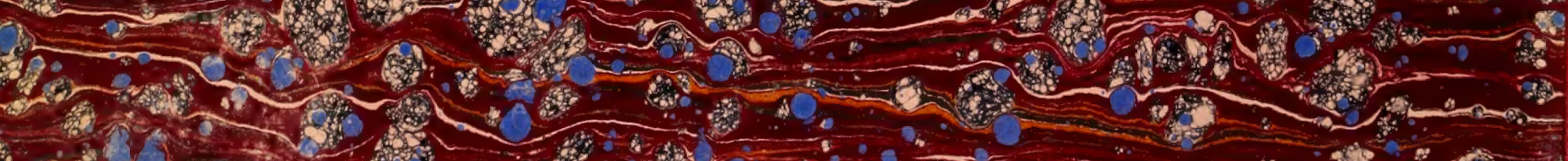
$100 \times 3=0$ $x+\cos 2=$ 